\title{
Organic Photoredox-Catalyzed Decarboxylative Trifluoromethylselenolation of Aliphatic Carboxylic Acids with $\left[\mathrm{Me}_{4} \mathrm{~N}\right]\left[\mathrm{SeCF}_{3}\right]$
}

\author{
Qiu-Yan Han, Kai-Li Tan, Hao-Nan Wang, Cheng-Pan Zhang* \\ School of Chemistry, Chemical Engineering and Life Science, Wuhan University of \\ Technology, 205 Luoshi Road, Wuhan 430070, China. \\ E-mail: cpzhang@whut.edu.cn, zhangchengpan1982@hotmail.com.
}

\section{Table of contents}

1. General information S2

2. Screening of the optimal reaction conditions for decarboxylative trifluoromethylselenolation of $\mathbf{1 a}$ by $\left[\mathrm{Me}_{4} \mathrm{~N}\right]\left[\mathrm{SeCF}_{3}\right]$ .S3

3. General procedure for photoredox-catalyzed decarboxylative trifluoromethylselenolation of aliphatic carboxylic acids $(\mathbf{1})$ by $\left[\mathrm{Me}_{4} \mathrm{~N}\right]\left[\mathrm{SeCF}_{3}\right] \ldots \mathrm{S} 10$

4. The control experiments for mechanistic insights . $\mathrm{S} 25$

5. The NMR spectra of the products (3). . $\mathrm{S} 39$ 


\section{General information}

All reactions were carried out under a nitrogen atmosphere with the visible-light irradiation. Blue LEDs $(10 \mathrm{~W} \times 2,435-455 \mathrm{~nm})$ were used for blue-light irradiation, and purple LEDs $(3 \mathrm{~W} \times 8,395-410 \mathrm{~nm}$ ) were used for purple-light irradiation. In each case, the distance between the light source and the reaction vessel was $3-7 \mathrm{~cm}$ (see the pictures below). A fan attached to the apparatus was used to maintain the temperature inside the "box" at room temperature. Unless otherwise specified, the NMR spectra were recorded in $\mathrm{CDCl}_{3}$ on a $500 \mathrm{MHz}$ (for ${ }^{1} \mathrm{H} \mathrm{NMR}$ ), $471 \mathrm{MHz}$ (for ${ }^{19} \mathrm{~F}$ NMR), and $126 \mathrm{MHz}$ (for ${ }^{13} \mathrm{C} \mathrm{NMR}$ ) spectrometer. All chemical shifts were reported in ppm relative to TMS for ${ }^{1} \mathrm{H}$ NMR (0 ppm) and $\mathrm{PhOCF}_{3}$ for ${ }^{19} \mathrm{~F}$ NMR $(-58.00 \mathrm{ppm})$ as an internal or external standard. The coupling constants were reported in Hertz $(\mathrm{Hz})$. The following abbreviations were used to explain the multiplicities: $\mathrm{s}=$ singlet, $\mathrm{d}=$ doublet, $\mathrm{t}=$ triplet, $\mathrm{q}=$ quartet, $\mathrm{m}=$ multiplet, $\mathrm{brs}=$ broad singlet. The HPLC experiments were carried out on a Wufeng LC-100 II instrument (column: Shodex, C18, $5 \mu \mathrm{m}, 4.6 \times 250 \mathrm{~mm}$ ), and the yields of the products were determined by using the corresponding pure compounds as the external standards. Melting points were measured and uncorrected. MS experiments were performed on a TOF-Q ESI or EI instrument. $\left[\mathrm{Me}_{4} \mathrm{~N}\right]\left[\mathrm{SeCF}_{3}\right]$ was prepared according to the literature. ${ }^{1}$ The photocatalysts $[\mathrm{Mes}-\mathrm{Acr}-\mathrm{Me}]\left[\mathrm{BF}_{4}\right]$ and $[\mathrm{Mes}-\mathrm{Acr}-\mathrm{Ph}]\left[\mathrm{BF}_{4}\right]$ were synthesized according to the literatures. ${ }^{2}$ Solvents were dried before use according to the literature. ${ }^{3}$ Other reagents in the reactions were all purchased from the commercial sources and used without further purification.

Pictures of the custom-made "light box" for the visible-light-irradiated reactions:
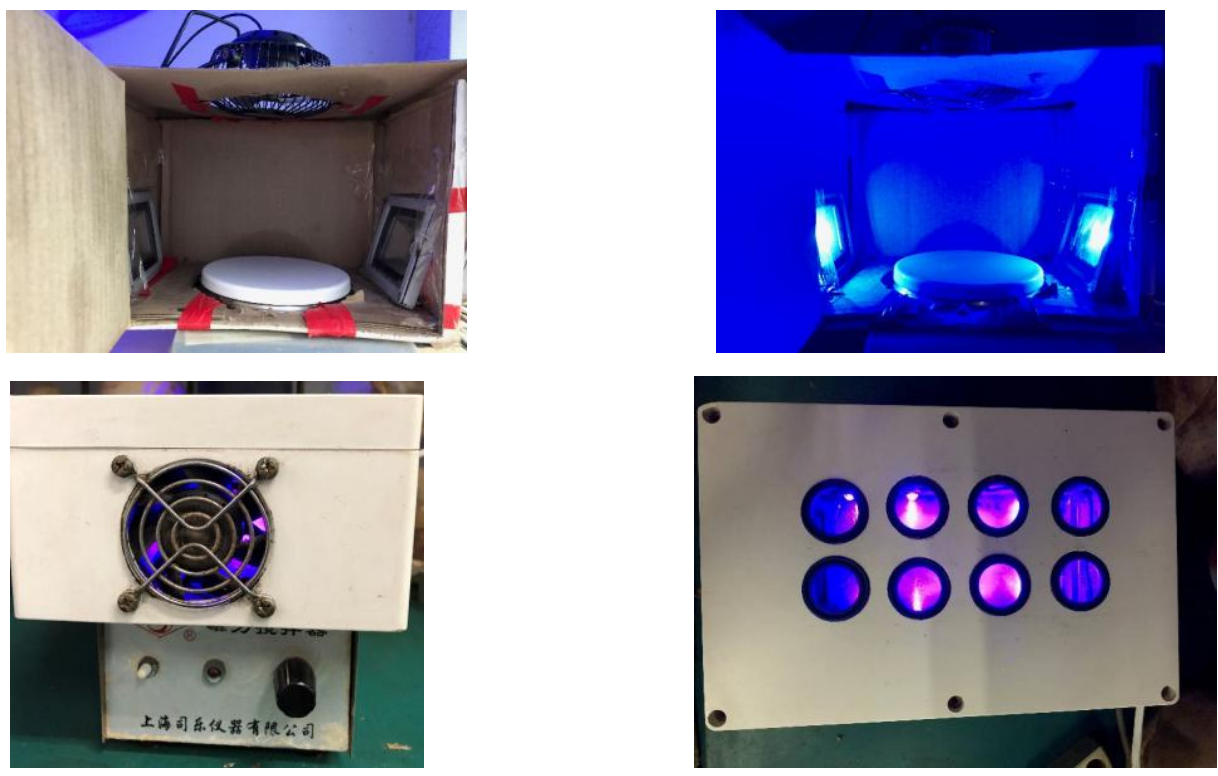
2. Screening of the optimal reaction conditions for decarboxylative trifluoromethylselenolation of $1 \mathrm{a}$ by $\left[\mathrm{Me}_{4} \mathrm{~N}\right]\left[\mathrm{SeCF}_{3}\right]$

Table S1 Photoredox-catalyzed decarboxylative trifluoromethylselenolation of $\mathbf{1 a}$ by $\left[\mathrm{Me}_{4} \mathrm{~N}\right]\left[\mathrm{SeCF}_{3}\right]$ in the presence of different catalysts. ${ }^{a}$

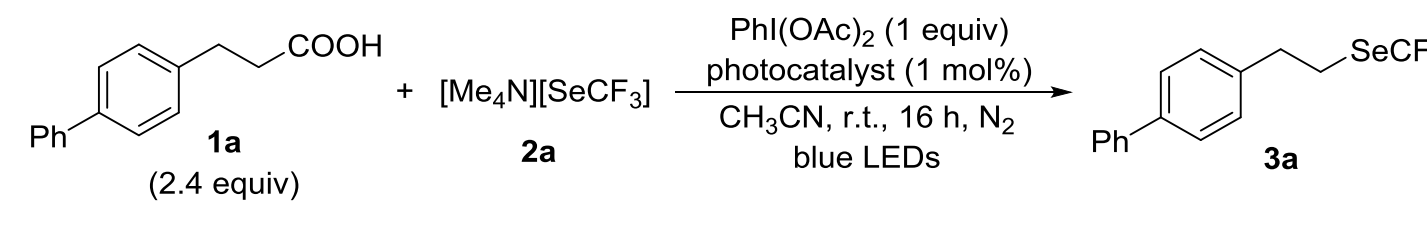

\begin{tabular}{|c|c|c|}
\hline Entry & Photocatalyst & Yield $(\mathbf{3 a}, \%)$ \\
\hline 1 & $\operatorname{Ir}(\text { ppy })_{3}$ & 9 \\
\hline 2 & {$\left[\operatorname{Ir}(\mathrm{ppy})_{2}(\mathrm{bpy})\right] \mathrm{PF}_{4}$} & trace \\
\hline 3 & {$\left[\operatorname{Ir}(\mathrm{ppy})_{2}(\mathrm{dtbbpy})\right] \mathrm{PF}_{6}$} & trace \\
\hline 4 & $\mathrm{Ru}(\mathrm{bpy})_{3} \mathrm{Cl}_{2} \cdot 6 \mathrm{H}_{2} \mathrm{O}$ & 5 \\
\hline 5 & $\mathrm{Ru}(\mathrm{bpy})_{3}\left(\mathrm{PF}_{6}\right)_{2}$ & 5 \\
\hline 6 & Eosin $Y$ & 2 \\
\hline 7 & benzophenone & 0 \\
\hline 8 & {$[\mathrm{Mes}-\mathrm{Acr}-\mathrm{Me}]\left[\mathrm{ClO}_{4}\right]$} & 46 \\
\hline 9 & {$[\mathrm{Mes}-\mathrm{Acr}-\mathrm{Me}]\left[\mathrm{BF}_{4}\right]$} & 51 \\
\hline 10 & {$[\mathrm{Mes}-\mathrm{Acr}-\mathrm{Ph}]\left[\mathrm{BF}_{4}\right]$} & 60 \\
\hline $11^{b}$ & {$[\mathrm{Mes}-\mathrm{Acr}-\mathrm{Ph}]\left[\mathrm{BF}_{4}\right]$} & 59 \\
\hline
\end{tabular}

${ }^{a}$ Reaction conditions: 1a $(0.48 \mathrm{mmol}), \mathbf{2 a}(0.20 \mathrm{mmol}), \mathrm{PhI}(\mathrm{OAc})_{2}(0.20 \mathrm{mmol})$, photocatalyst $(0.002 \mathrm{mmol}), \mathrm{CH}_{3} \mathrm{CN}(2 \mathrm{~mL})$, r.t., $\mathrm{N}_{2}, 16 \mathrm{~h}$, blue LEDs $(10 \mathrm{~W} \times 2$, 435-455 nm). The yields were determined by HPLC using 3a as an external standard $\left(\mathrm{t}_{\mathrm{R}}=8.34 \mathrm{~min}, \lambda_{\max }=253 \mathrm{~nm}\right.$, water/methanol $\left.(\mathrm{v} / \mathrm{v})=10: 90\right) . \quad{ }^{b}$ LEDs $(5 \mathrm{~W} \times 2$, $365 \mathrm{~nm})$.

Table S2 Photoredox-catalyzed decarboxylative trifluoromethylselenolation of $\mathbf{1 a}$ by $\left[\mathrm{Me}_{4} \mathrm{~N}\right]\left[\mathrm{SeCF}_{3}\right]$ in the presence of diverse oxidants. ${ }^{a}$ 


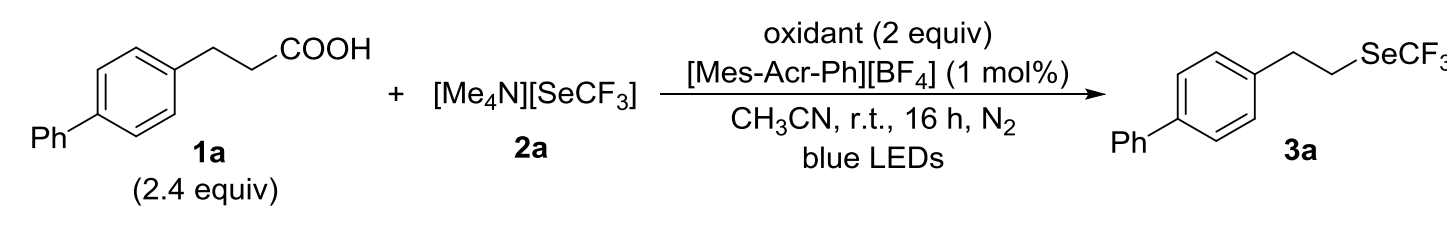

\begin{tabular}{|c|c|c|}
\hline Entry & Oxidant & Yield $(\mathbf{3 a}, \%)$ \\
\hline $1^{b}$ & $\mathrm{PhI}(\mathrm{OAc})_{2}$ & 60 \\
\hline 2 & $\operatorname{PhI}(\mathrm{OAc})_{2}$ & 68 \\
\hline 3 & $\mathrm{PhI}(\mathrm{TFA})_{2}$ & 51 \\
\hline 4 & NFSI & 84 \\
\hline 5 & $m-\mathrm{CPBA}$ & 37 \\
\hline 6 & DMP & 25 \\
\hline 7 & $\mathrm{H}_{2} \mathrm{O}_{2}(30 \% \mathrm{aq})$ & 24 \\
\hline 8 & TBHP (70\% aq) & 25 \\
\hline 9 & Selectfluor & 3 \\
\hline 10 & NIS & 0 \\
\hline 11 & NBS & trace \\
\hline 12 & NCS & trace \\
\hline 14 & DDQ & 0 \\
\hline 14 & $\mathrm{~K}_{2} \mathrm{~S}_{2} \mathrm{O}_{8}$ & 0 \\
\hline 15 & $\mathrm{AgNO}_{3}$ & 1 \\
\hline 16 & $\mathrm{KMnO}_{4}$ & 0 \\
\hline
\end{tabular}

${ }^{a}$ Reaction conditions: 1a $(0.48 \mathrm{mmol}), 2 \mathbf{2 a}(0.20 \mathrm{mmol})$, oxidant $(0.40 \mathrm{mmol})$, [Mes-Acr-Ph][BF $]$ (0.002 mmol), $\mathrm{CH}_{3} \mathrm{CN}(2 \mathrm{~mL})$, r.t., $\mathrm{N}_{2}, 16 \mathrm{~h}$, blue LEDs $(10 \mathrm{~W} \times$ 2, 435-455 nm). The yields were determined by HPLC using 3a as an external standard $\left(\mathrm{t}_{\mathrm{R}}=8.34 \mathrm{~min}, \lambda_{\max }=253 \mathrm{~nm}\right.$, water/methanol $\left.(\mathrm{v} / \mathrm{v})=10: 90\right) . \quad b$ $\mathrm{PhI}(\mathrm{OAc})_{2}(0.20 \mathrm{mmol})$.

Table S3 The solvent effects on the photoredox-catalyzed reactions of $\mathbf{1 a}$ and $\mathbf{2 a}$ at 
room temperature. ${ }^{a}$

Solvent
Entry
$\begin{gathered}\text { (2.4 equiv) } \\ \text { blue LEDs }\end{gathered}$

1

2

3

4

5

6

7

8

9

10

11

12
$\mathrm{CH}_{3} \mathrm{CN}$

$\mathrm{CH}_{3} \mathrm{CN} / \mathrm{H}_{2} \mathrm{O}(10: 1)$

acetone $/ \mathrm{H}_{2} \mathrm{O}(10: 1)$

acetone

DCM

DCE

toluene

THF

1,4-dioxane

DMSO

NMP

DMF
84

9

16

34

68

74

57

23

7

0

trace

trace

$\bar{a}$ Reaction conditions: 1a $(0.48 \mathrm{mmol}), 2 \mathbf{2 a}(0.20 \mathrm{mmol})$, NFSI $(0.40 \mathrm{mmol})$, [Mes-Acr-Ph][BF $]$ (0.002 mmol), solvent $(2 \mathrm{~mL})$, r.t., $\mathrm{N}_{2}, 16 \mathrm{~h}$, blue LEDs $(10 \mathrm{~W} \times 2$, 435-455 nm). The yields were determined by HPLC using 3a as an external standard $\left(\mathrm{t}_{\mathrm{R}}=8.34 \mathrm{~min}, \lambda_{\max }=253 \mathrm{~nm}\right.$, water/methanol $\left.(\mathrm{v} / \mathrm{v})=10: 90\right)$.

Table S4 Photoredox-catalyzed decarboxylative trifluoromethylselenolation of $\mathbf{1 a}$ by 2a with different reactant ratios. ${ }^{a}$

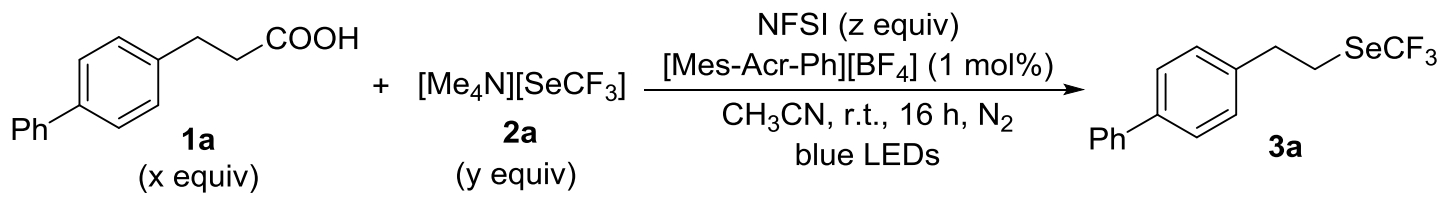




\begin{tabular}{|c|c|c|}
\hline Entry & $x: y: z$ & Yield $(\mathbf{3 a}, \%)$ \\
\hline 1 & $3.0: 1.0: 2.0$ & 84 \\
\hline 2 & $2.4: 1.0: 2.0$ & 84 \\
\hline 3 & $2.0: 1.0: 2.0$ & 82 \\
\hline 4 & $1.7: 1.0: 2.0$ & 82 \\
\hline 5 & $1.5: 1.0: 2.0$ & 80 \\
\hline 6 & $1.5: 1.0: 2.5$ & 49 \\
\hline 7 & $1.5: 1.0: 1.5$ & 73 \\
\hline 8 & $1.5: 1.0: 1.0$ & 70 \\
\hline 9 & $1.2: 1.0: 2.0$ & 68 \\
\hline 10 & $1.2: 1.0: 1.5$ & 78 \\
\hline 11 & $1.2: 1.0: 1.2$ & 79 \\
\hline 12 & $1.3: 1.0: 1.3$ & 83 \\
\hline 13 & $1.3: 1.0: 1.5$ & 84 \\
\hline 14 & $1.0: 1.0: 1.0$ & 56 \\
\hline 15 & $1.0: 1.0: 2.0$ & 58 \\
\hline 16 & $1.0: 1.2: 2.0$ & 75 \\
\hline 17 & $1.0: 1.5: 2.0$ & 77 \\
\hline 18 & $1.0: 2.0: 2.0$ & 55 \\
\hline 19 & $1.0: 2.0: 1.5$ & 24 \\
\hline 20 & $1.0: 2.0: 1.0$ & 14 \\
\hline 21 & $1.0: 1.5: 1.5$ & 56 \\
\hline 22 & $1.0: 1.5: 1.0$ & 19 \\
\hline 23 & $1.5: 1.5: 1.0$ & 21 \\
\hline 24 & $2.0: 2.0: 1.0$ & 5 \\
\hline
\end{tabular}


${ }^{a}$ Reaction conditions: 1a $(0.20,0.24,0.26,0.30,0.34,0.40,0.48,0.60 \mathrm{mmol}), 2 \mathbf{a}$ $(0.20,0.24,0.30,0.40 \mathrm{mmol})$, NFSI $(0.20,0.24,0.26,0.30,0.40,0.50 \mathrm{mmol})$, [Mes-Acr-Ph] $\left[\mathrm{BF}_{4}\right](0.002 \mathrm{mmol}), \mathrm{CH}_{3} \mathrm{CN}(2 \mathrm{~mL})$, r.t., $\mathrm{N}_{2}, 16 \mathrm{~h}$, blue LEDs $(10 \mathrm{~W} \times$ 2, 435-455 $\mathrm{nm}$ ). The yields were determined by HPLC using 3a as an external standard $\left(\mathrm{t}_{\mathrm{R}}=8.34 \mathrm{~min}, \lambda_{\max }=253 \mathrm{~nm}\right.$, water $/$ methanol $\left.(\mathrm{v} / \mathrm{v})=10: 90\right)$.

Table S5 Photoredox-catalyzed decarboxylative trifluoromethylselenolation of 1a by $\mathbf{2 a}$ with different reaction times and catalyst loadings. ${ }^{a}$

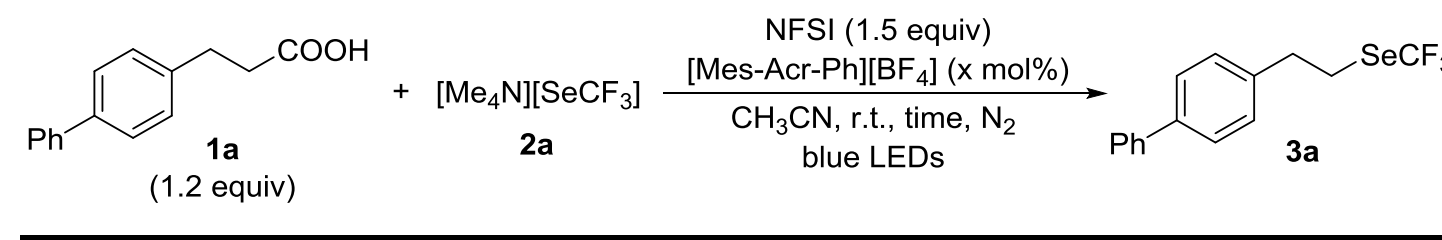

\begin{tabular}{|c|c|c|c|}
\hline Entry & $\mathrm{x} \mathrm{mol} \%$ & Time (h) & Yield $(\mathbf{3 a}, \%)$ \\
\hline 1 & 1 & 4 & 32 \\
\hline 2 & 1 & 8 & 49 \\
\hline 3 & 1 & 12 & 63 \\
\hline 4 & 1 & 16 & 78 \\
\hline 5 & 1 & 24 & 85 \\
\hline 6 & 0.5 & 24 & 80 \\
\hline 7 & 0.1 & 24 & 49 \\
\hline 8 & 2 & 24 & 84 \\
\hline $9^{b}$ & 1 & 24 & $86(84)$ \\
\hline $10^{b}$ & 1 & 36 & 87 \\
\hline $11^{b, c}$ & 1 & 24 & 0 \\
\hline $12^{b, d}$ & 1 & 24 & 0 \\
\hline $13^{b, e}$ & 1 & 24 & 0 \\
\hline
\end{tabular}

${ }^{a}$ Reaction conditions: 1a $(0.24 \mathrm{mmol}), 2 \mathbf{2 a}(0.20 \mathrm{mmol})$, NFSI $(0.30 \mathrm{mmol})$, [Mes-Acr-Ph][BF $]$ (0.0002, 0.001, 0.002, $0.004 \mathrm{mmol}), \mathrm{CH}_{3} \mathrm{CN}(2 \mathrm{~mL})$, r.t., $\mathrm{N}_{2}$, time, blue LEDs $(10 \mathrm{~W} \times 2,435-455 \mathrm{~nm})$. The yields were determined by HPLC using 3a 
as an external standard $\left(t_{\mathrm{R}}=8.34 \mathrm{~min}, \lambda_{\max }=253 \mathrm{~nm}\right.$, water/methanol $\left.(\mathrm{v} / \mathrm{v})=10: 90\right)$. Isolated yield is depicted in the parenthesis. ${ }^{b} \mathbf{1 a}(0.26 \mathrm{mmol}), \mathbf{2 a}(0.20 \mathrm{mmol})$, NFSI $(0.26 \mathrm{mmol}),[\mathrm{Mes}-\mathrm{Acr}-\mathrm{Ph}]\left[\mathrm{BF}_{4}\right](0.002 \mathrm{mmol}), \mathrm{CH}_{3} \mathrm{CN}(2 \mathrm{~mL})$, r.t., $\mathrm{N}_{2}$, time, blue LEDs $(10 \mathrm{~W} \times 2,435-455 \mathrm{~nm}) . \quad{ }^{c}$ The reaction was conducted in the darkness. ${ }^{d}$ Without oxidant. $\quad{ }^{e}$ Without photocatalyst.

Table S6 Photoredox-catalyzed decarboxylative trifluoromethylselenolation of 8-phenyloctanoic acid (1x) by $\mathbf{2 a}$ under different reaction conditions. ${ }^{a}$

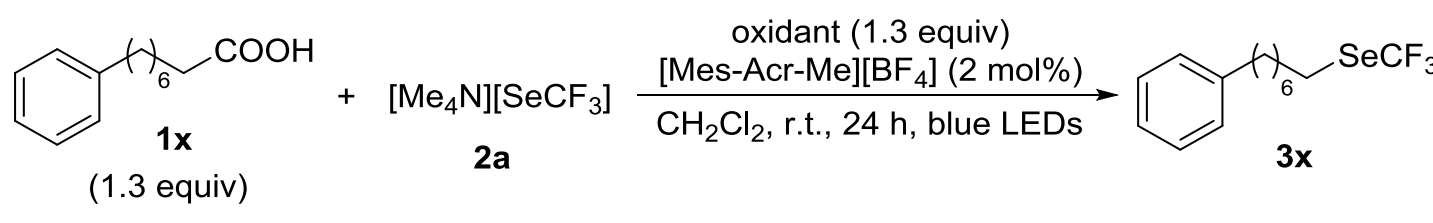

\begin{tabular}{|c|c|c|}
\hline Entry & Oxidant & Yield $(\mathbf{3 x}, \%)$ \\
\hline 1 & NFSI & 6 \\
\hline $2^{b}$ & NFSI & 6 \\
\hline 3 & $m-\mathrm{CPBA}$ & 6 \\
\hline 4 & $\mathrm{H}_{2} \mathrm{O}_{2}(50 \% \mathrm{aq})$ & 14 \\
\hline 5 & TBHP (70\% aq) & 14 \\
\hline 6 & $\mathrm{AgO}_{2}$ & 15 \\
\hline 7 & $\mathrm{~K}_{2} \mathrm{~S}_{2} \mathrm{O}_{8}$ & 2 \\
\hline 8 & Selectfluor & 7 \\
\hline 9 & $\mathrm{KMnO}_{4}$ & 4 \\
\hline 10 & NMO & 1 \\
\hline 11 & LPO & 19 \\
\hline 12 & $\mathrm{PhI}(\mathrm{TFA})_{2}$ & 21 \\
\hline 13 & $\mathrm{PhI}(\mathrm{OAc})_{2}$ & 22 \\
\hline $14^{c}$ & $\mathrm{PhI}(\mathrm{OAc})_{2}$ & 47 \\
\hline $14^{c, d}$ & $\mathrm{PhI}(\mathrm{OAc})_{2}$ & 38 \\
\hline
\end{tabular}




$\begin{array}{ccc}16^{c, e} & \operatorname{PhI}(\mathrm{OAc})_{2} & 31 \\ 19^{\mathrm{c} . f} & \operatorname{PhI}(\mathrm{OAc})_{2} & 49 \\ 20^{\mathrm{c}, g} & \operatorname{PhI}(\mathrm{OAc})_{2} & 63 \\ \mathbf{2 1}^{\mathrm{c}, \boldsymbol{h}} & \text { PhI }(\mathbf{O A c})_{2} & \mathbf{6 5}(\mathbf{5 4})\end{array}$

$\bar{a}$ Reaction conditions: 1x (0.26 mmol), 2a (0.20 mmol), PhI (OAc) 2 (0.26 mmol), [Mes-Acr-Me][BF 4 (0.004 mmol), $\mathrm{CH}_{2} \mathrm{Cl}_{2}(2 \mathrm{~mL})$, r.t., $\mathrm{N}_{2}$, blue LEDs $(10 \mathrm{~W} \times 2$, 435-455 nm), $24 \mathrm{~h}$. The yields were determined by HPLC using $\mathbf{3 x}$ as an external standard $\left(\mathrm{t}_{\mathrm{R}}=12.53 \mathrm{~min}, \lambda_{\max }=214 \mathrm{~nm}\right.$, water $/$ methanol $\left.(\mathrm{v} / \mathrm{v})=10: 90\right)$. Isolated yield is depicted in the parenthesis. ${ }^{b} \mathrm{CH}_{3} \mathrm{CN}$ was used instead of $\mathrm{CH}_{2} \mathrm{Cl}_{2}$. ${ }^{c}$ Purple LEDs $(3 \mathrm{~W} \times 8,395-410 \mathrm{~nm})$ was used instead of blue LEDs. $\quad{ }^{d}$ [Mes-Me-Acr] $\left[\mathrm{BF}_{4}\right]$ $(0.002 \mathrm{mmol}) . \quad{ }^{e}$ [Mes-Acr-Ph] $\left[\mathrm{BF}_{4}\right]$ was used instead of [Mes-Acr-Me] $\left[\mathrm{BF}_{4}\right] . \quad f$ 48 h. $\quad{ }^{g} 6$ days. $\quad{ }^{h} 7$ days.

Table S7 Photoredox-catalyzed decarboxylative trifluoromethylselenolation of $\mathbf{1 a}$ by $\mathbf{2 a}$ in the presence of different bases. ${ }^{a}$

$\begin{gathered}1 \mathbf{a} \\ (1.3 \text { equiv })\end{gathered}$
Entry

\begin{tabular}{cccc}
\hline 1 & none & NFSI & 86 \\
2 & $\mathrm{NaHCO}_{3}$ & NFSI & 68 \\
3 & $\mathrm{~K}_{2} \mathrm{HPO}_{4}$ & NFSI & 35 \\
4 & $\mathrm{~K}_{3} \mathrm{PO}_{4}$ & NFSI & trace \\
5 & $\mathrm{NaOAc}$ & NFSI & 55 \\
6 & $\mathrm{KF}$ & NFSI & 70 \\
$7^{b}$ & none & PhI(OAc) & 36 \\
8 & none & PhI $(\mathrm{OAc})_{2}$ & 43
\end{tabular}




$\begin{array}{cccc}9 & \mathrm{NaHCO}_{3} & \mathrm{PhI}(\mathrm{OAc})_{2} & 43 \\ 10 & \mathrm{~K}_{2} \mathrm{HPO}_{4} & \mathrm{PhI}(\mathrm{OAc})_{2} & 12 \\ 11 & \mathrm{~K}_{3} \mathrm{PO}_{4} & \mathrm{PhI}(\mathrm{OAc})_{2} & 13 \\ 12 & \mathrm{NaOAc} & \mathrm{PhI}(\mathrm{OAc})_{2} & 31\end{array}$

\begin{abstract}
${ }^{a}$ Reaction conditions: 1a $(0.26 \mathrm{mmol}), 2 \mathrm{2a}(0.20 \mathrm{mmol})$, oxidant $(0.26 \mathrm{mmol})$, [Mes-Acr-Ph] $\left[\mathrm{BF}_{4}\right](0.002 \mathrm{mmol}), \mathrm{CH}_{3} \mathrm{CN}(2 \mathrm{~mL})$, r.t., $\mathrm{N}_{2}, 24 \mathrm{~h}$, blue LEDs (10 W $\times$ 2, 435-455 $\mathrm{nm}$ ). The yields were determined by HPLC using 3a as an external standard $\left(t_{R}=8.34 \mathrm{~min}, \lambda_{\max }=253 \mathrm{~nm}\right.$, water/methanol $\left.(\mathrm{v} / \mathrm{v})=10: 90\right) . \quad{ }^{b} 16 \mathrm{~h}$.
\end{abstract}

\title{
3. General procedure for photoredox-catalyzed decarboxylative trifluoromethylselenolation of aliphatic carboxylic acids (1) by $\left[\mathrm{Me}_{4} \mathrm{~N}\right]\left[\mathrm{SeCF}_{3}\right]$.
}

In a nitrogen-filled glove box, a sealed tube was charged with aliphatic carboxylic acid (1, $0.26 \mathrm{mmol})$, NFSI (82.0 mg, $0.26 \mathrm{mmol})$ or $\mathrm{PhI}(\mathrm{OAc})_{2}(83.7 \mathrm{mg}, 0.26 \mathrm{mmol})$, $\left[\mathrm{Me}_{4} \mathrm{~N}\right]\left[\mathrm{SeCF}_{3}\right](44.4 \mathrm{mg}, 0.2 \mathrm{mmol})$, [Mes-Acr-Ph][BF $]$ (1.0 or 2.0 or $5.0 \mathrm{mg}, 0.002$ or 0.004 or $0.01 \mathrm{mmol}$ ) or [Mes-Acr-Me][BF $]$ (1.6 or 4.0 or $8.0 \mathrm{mg}, 0.004$ or 0.01 or $0.02 \mathrm{mmol}$ ), and $\mathrm{CH}_{3} \mathrm{CN}$ or $\mathrm{CH}_{2} \mathrm{Cl}_{2}(2 \mathrm{~mL})$ with vigorous stirring. The reaction mixture was irradiated by $2 \times 10 \mathrm{~W}$ blue LEDs or $8 \times 3 \mathrm{~W}$ purple LEDs $(3-7 \mathrm{~cm}$ away, with cooling fan to keep the reaction at room temperature) for 24 hours, quenched with water ( 2 drops, approximately $120 \mathrm{mg}$ ) and concentrated under reduced pressure. The residue was purified by flash column chromatography on silica gel using petroleum ether or a mixture of petroleum ether and ethyl acetate as eluents to give the trifluoromethylselenolated products $(\mathbf{3})$.<smiles>FC(F)(F)OCCc1ccc(-c2ccccc2)cc1</smiles>

(2-([1,1'-Biphenyl]-4-yl)ethyl)(trifluoromethyl)selane (3a). White solid (55.1 mg, $84 \%$ yield), petroleum ether as eluent for column chromatography. M.p.: $40-42{ }^{\circ} \mathrm{C} .{ }^{1} \mathrm{H}$ NMR (500 MHz, $\left.\mathrm{CDCl}_{3}\right) \delta 7.60(\mathrm{~d}, J=8.2 \mathrm{~Hz}, 2 \mathrm{H}), 7.57(\mathrm{~d}, J=8.2 \mathrm{~Hz}, 2 \mathrm{H}), 7.45$ (t, $J=7.5 \mathrm{~Hz}, 2 \mathrm{H}), 7.36(\mathrm{t}, J=7.4 \mathrm{~Hz}, 1 \mathrm{H}), 7.29(\mathrm{~d}, J=8.2 \mathrm{~Hz}), 3.27(\mathrm{t}, J=7.2 \mathrm{~Hz}, 2 \mathrm{H})$, $3.16(\mathrm{t}, J=7.3 \mathrm{~Hz}, 2 \mathrm{H}) .{ }^{19} \mathrm{~F}$ NMR $\left(471 \mathrm{MHz}, \mathrm{CDCl}_{3}\right) \delta-33.9(\mathrm{~s}, 3 \mathrm{~F}) .{ }^{13} \mathrm{C}$ NMR $(126$ $\left.\mathrm{MHz}, \mathrm{CDCl}_{3}\right) \delta 140.8,139.9,138.8,128.9,128.8,127.5,127.3,127.1,122.8(\mathrm{q}, J=$ $331.4 \mathrm{~Hz}$ ), 36.4, 26.6 (q, $J=1.1 \mathrm{~Hz})$. IR (KBr): 3058, 3030, 2949, 2875, 2860, 1598, 
1564, 1523, 1488, 1450, 1408, 1315, 1273, 1213, 1199, 1147, 1095, 1005, 950, 914, 846, 821, 766, 738, 692, $640 \mathrm{~cm}^{-1}$. HRMS-ESI (m/z) calcd. for $\mathrm{C}_{15} \mathrm{H}_{13} \mathrm{~F}_{3} \mathrm{NaSe}([\mathrm{M}+$ $\mathrm{Na}^{+}$): 356.0062; found: 356.0065 .<smiles>COc1ccc(CC[Se]C(F)(F)F)cc1</smiles>

(4-Methoxyphenethyl)(trifluoromethyl)selane (3b). Light yellow liquid (33.6 mg, $59 \%$ yield), petroleum ether as eluent for column chromatography. ${ }^{1} \mathrm{H}$ NMR (500 $\left.\mathrm{MHz} \mathrm{CDCl}_{3}\right) \delta 7.13(\mathrm{~d}, J=8.5 \mathrm{~Hz}, 2 \mathrm{H}), 6.87(\mathrm{~d}, J=8.5 \mathrm{~Hz}, 2 \mathrm{H}), 3.81(\mathrm{~s}, 3 \mathrm{H}), 3.20(\mathrm{t}$, $J=7.4 \mathrm{~Hz}, 2 \mathrm{H}), 3.05(\mathrm{t}, J=7.5 \mathrm{~Hz}, 2 \mathrm{H}) .{ }^{19} \mathrm{~F} \mathrm{NMR}\left(471 \mathrm{MHz}, \mathrm{CDCl}_{3}\right) \delta-34.0(\mathrm{~s}, 3 \mathrm{~F})$. ${ }^{13} \mathrm{C} \mathrm{NMR}\left(126 \mathrm{MHz}, \mathrm{CDCl}_{3}\right) \delta 158.5,131.9,129.4,122.8(\mathrm{q}, J=331.4 \mathrm{~Hz}), 114.1$, 55.3, 35.8, 27.1. IR (KBr): 3033, 3001, 2955, 2936, 2916, 2837, 1612, 1584, 1513, 1466, 1442, 1302, 1267, 1249, 1212, 1179, 1101, 1075, 1037, 872, 844, 820, 760, 738, $696 \mathrm{~cm}^{-1}$. HRMS-ESI $(\mathrm{m} / \mathrm{z})$ calcd. for $\mathrm{C}_{10} \mathrm{H}_{12} \mathrm{~F}_{3} \mathrm{OSe}\left([\mathrm{M}+\mathrm{H}]^{+}\right): 283.0008$; found: 283.0005 .<smiles>COc1ccccc1C[Ge]C(F)(F)C(F)(F)F</smiles>

(2-Methoxybenzyl)(trifluoromethyl)selane (3c). Light yellow liquid (32.7 mg, 61\% yield), petroleum ether as eluent for column chromatography. ${ }^{1} \mathrm{H}$ NMR (500 MHz, $\left.\mathrm{CDCl}_{3}\right) \delta 7.29-7.25(\mathrm{~m}, 2 \mathrm{H}), 6.91(\mathrm{t}, J=7.5 \mathrm{~Hz}, 1 \mathrm{H}), 6.89(\mathrm{~d}, J=8.2 \mathrm{~Hz}, 1 \mathrm{H}), 4.20(\mathrm{~s}$, 2H), 3.87 (s, 3H). ${ }^{19} \mathrm{~F}$ NMR $\left(471 \mathrm{MHz}, \mathrm{CDCl}_{3}\right) \delta-34.7$ (s, 3F). ${ }^{13} \mathrm{C}$ NMR $(126 \mathrm{MHz}$, $\left.\mathrm{CDCl}_{3}\right) \delta 157.3,130.0,129.3,125.5,123.2(\mathrm{q}, J=331.4 \mathrm{~Hz}), 120.6,110.6,55.4,24.0$ (q, $J=1.7 \mathrm{~Hz}) . \mathrm{IR}(\mathrm{KBr}): 3009,2961,2941,2840,1602,1590,1494,1466,1439$, 1318, 1293, 1250, 1219, 1197, 1096, 1074, 1048, 1028, 933, 846, 752, 738, $611 \mathrm{~cm}^{-1}$. HRMS-ESI (m/z) calcd. for $\mathrm{C}_{9} \mathrm{H}_{10} \mathrm{~F}_{3} \mathrm{OSe}\left([\mathrm{M}+\mathrm{H}]^{+}\right)$: 270.9843 ; found: 270.9845 .<smiles>COc1cccc(C[Se]C(F)(F)F)c1</smiles>

(3-Methoxybenzyl)(trifluoromethyl)selane (3d). ${ }^{4 \mathrm{a}}$ Light yellow liquid (40.9 mg, $76 \%$ yield), petroleum ether as eluent for column chromatography. ${ }^{1} \mathrm{H}$ NMR (500 MHz, $\left.\mathrm{CDCl}_{3}\right) \delta 7.25(\mathrm{t}, J=7.9 \mathrm{~Hz}, 1 \mathrm{H}), 6.93(\mathrm{~d}, J=7.6 \mathrm{~Hz}, 1 \mathrm{H}), 6.88(\mathrm{~m}, 1 \mathrm{H}), 6.83(\mathrm{dd}, J$ $=8.2,1.8 \mathrm{~Hz}, 1 \mathrm{H}), 4.23(\mathrm{~s}, 2 \mathrm{H}), 3.81(\mathrm{~s}, 3 \mathrm{H}) .{ }^{19} \mathrm{~F} \mathrm{NMR}\left(471 \mathrm{MHz}, \mathrm{CDCl}_{3}\right) \delta-34.5(\mathrm{~s}$, 
3F). ${ }^{13} \mathrm{C} \mathrm{NMR}\left(126 \mathrm{MHz}, \mathrm{CDCl}_{3}\right) \delta 159.9,137.5,129.9,122.8(\mathrm{q}, J=331.3 \mathrm{~Hz}$ ), $121.3,114.5,113.4,55.2,29.1(\mathrm{q}, J=1.4 \mathrm{~Hz})$.<smiles>COc1ccc(C[Se]C(F)(F)F)cc1</smiles>

(4-Methoxybenzyl)(trifluoromethyl)selane (3e) ${ }^{4 \mathrm{~b}}$ Light yellow liquid (43.0 mg, 80\% yield), petroleum ether as eluent for column chromatography. ${ }^{1} \mathrm{H}$ NMR (500 MHz, $\left.\mathrm{CDCl}_{3}\right) \delta 7.29(\mathrm{~d}, J=8.5 \mathrm{~Hz}, 2 \mathrm{H}), 6.88(\mathrm{~d}, J=8.6 \mathrm{~Hz}, 2 \mathrm{H}), 4.26(\mathrm{~s}, 2 \mathrm{H}), 3.83(\mathrm{~s}, 3 \mathrm{H})$. ${ }^{19} \mathrm{~F} \mathrm{NMR}\left(471 \mathrm{MHz}, \mathrm{CDCl}_{3}\right) \delta-34.4(\mathrm{~s}, 3 \mathrm{~F}) .{ }^{13} \mathrm{C} \mathrm{NMR}\left(126 \mathrm{MHz}, \mathrm{CDCl}_{3}\right) \delta 159.3$, 130.2, 127.8, 122.9 (q, $J=331.2 \mathrm{~Hz}), 114.3,55.3,28.8(\mathrm{q}, J=1.4 \mathrm{~Hz})$.<smiles>FC(F)(F)[Ge](Cc1cccc(Oc2ccccc2)c1)(Oc1ccccc1)C(F)(F)F</smiles>

(3-Phenoxybenzyl)(trifluoromethyl)selane (3f). Light yellow liquid (29.9 mg, 45\%), petroleum ether as eluent for column chromatography. ${ }^{1} \mathrm{H} \mathrm{NMR}\left(500 \mathrm{MHz}, \mathrm{CDCl}_{3}\right) \delta$ $7.36(\mathrm{t}, J=7.5 \mathrm{~Hz}, 2 \mathrm{H}), 7.29(\mathrm{t}, J=7.9 \mathrm{~Hz}, 1 \mathrm{H}), 7.14(\mathrm{t}, J=7.4 \mathrm{~Hz}, 1 \mathrm{H}), 7.08(\mathrm{~d}, J=$ $7.6 \mathrm{~Hz}, 1 \mathrm{H}), 7.03-7.00(\mathrm{~m}, 3 \mathrm{H}), 6.92(\mathrm{dd}, J=8.1,1.8 \mathrm{~Hz}, 1 \mathrm{H}), 4.21(\mathrm{~s}, 2 \mathrm{H}) .{ }^{19} \mathrm{~F} \mathrm{NMR}$ $\left(471 \mathrm{MHz}, \mathrm{CDCl}_{3}\right) \delta-34.4$ (s, 3F). ${ }^{13} \mathrm{C} \mathrm{NMR}\left(126 \mathrm{MHz}, \mathrm{CDCl}_{3}\right) \delta 157.7,156.8,138.1$, $130.2,129.8,123.7,123.6,122.8$ (q, $J=332.0 \mathrm{~Hz}), 119.2,119.1,118.0,28.8$ (q, $J=$ $1.2 \mathrm{~Hz})$. IR (KBr): 3041, 2927, 2855, 1585, 1487, 1456, 1445, 1310, 1258, 1219 , 1125, 1098, 1073, 1023, 956, 882, 816, 785, 760, 738, $693 \mathrm{~cm}^{-1}$. HRMS-ESI (m/z) calcd. for $\mathrm{C}_{14} \mathrm{H}_{12} \mathrm{~F}_{3} \mathrm{OSe}\left([\mathrm{M}+\mathrm{H}]^{+}\right)$: 333.0000; found: 332.9993 .<smiles>FC(F)(F)C(F)(F)C(F)(F)Cc1ccc(-c2ccccc2)cc1</smiles>

([1,1'-Biphenyl]-4-ylmethyl)(trifluoromethyl)selane (3g). ${ }^{4 \mathrm{~b}}$ White solid (33.4 mg, 53\% yield), petroleum ether as eluent for column chromatography. M.p.: $70-72{ }^{\circ} \mathrm{C} .{ }^{1} \mathrm{H}$ $\operatorname{NMR}\left(500 \mathrm{MHz}, \mathrm{CDCl}_{3}\right) \delta$ 7.61-7.57 (m, 4H), 7.48-7.42 (m, 4H), 7.37 (t, J= 7.3 Hz, 1H), 4.31 (s, 2H). ${ }^{19} \mathrm{~F}$ NMR (471 MHz, $\left.\mathrm{CDCl}_{3}\right) \delta-34.3$ (s, 3F). ${ }^{13} \mathrm{C}$ NMR $(126 \mathrm{MHz}$, $\left.\mathrm{CDCl}_{3}\right) \delta 140.8,140.5,135.1,129.5,128.8,127.6,127.5,127.1,122.9$ (q, $J=332.6$ $\mathrm{Hz}), 28.9$ (q, $J=1.5 \mathrm{~Hz}$ ). 
$\mathrm{SeCF}_{3}$

(4-(Methylsulfonyl)benzyl)(trifluoromethyl)selane (3h). White solid $(29.4 \mathrm{mg}, 46 \%$ yield), a mixture of petroleum ether/ethyl acetate $=2: 1(\mathrm{v} / \mathrm{v})$ as eluents for column chromatography. M.p.: $79-81{ }^{\circ} \mathrm{C} .{ }^{1} \mathrm{H}$ NMR $\left(500 \mathrm{MHz} \mathrm{CDCl}_{3}\right) \delta 7.91(\mathrm{~d}, J=8.2 \mathrm{~Hz}$, $2 \mathrm{H}), 7.54(\mathrm{~d}, J=8.2 \mathrm{~Hz}, 2 \mathrm{H}), 4.27(\mathrm{~s}, 2 \mathrm{H}), 3.06(\mathrm{~s}, 3 \mathrm{H}) .{ }^{19} \mathrm{~F}$ NMR $\left(471 \mathrm{MHz}, \mathrm{CDCl}_{3}\right)$ $\delta-34.2(\mathrm{~s}, 3 \mathrm{~F}) .{ }^{13} \mathrm{C}$ NMR $\left(126 \mathrm{MHz}, \mathrm{CDCl}_{3}\right) \delta 143.1,139.9,129.9,128.0,122.5$ (q, $J$ $=331.6 \mathrm{~Hz}), 44.5,28.1$ (q, $J=1.6 \mathrm{~Hz}) . \mathrm{IR}(\mathrm{KBr}): 3013,2932,1596,1491,1447,1409$, 1326, 1300, 1219, 1200, 1147, 1098, 1018, 963, 843, 817, 765, 738, 729, 645, 622, $608 \mathrm{~cm}^{-1}$. HRMS-ESI $(\mathrm{m} / \mathrm{z})$ calcd. for $\mathrm{C}_{9} \mathrm{H}_{10} \mathrm{~F}_{3} \mathrm{O}_{2} \mathrm{SSe}\left([\mathrm{M}+\mathrm{H}]^{+}\right): 318.9513$; found: 318.9508 .

$\mathrm{SeCF}_{3}$

(4-(Phenoxymethyl)benzyl)(trifluoromethyl)selane (3i). White solid (56.5 mg, 82\% yield), petroleum ether as eluent for column chromatography. M.p.: $60-62{ }^{\circ} \mathrm{C} .{ }^{1} \mathrm{H}$ NMR (500 MHz, $\left.\mathrm{CDCl}_{3}\right) \delta 7.46(\mathrm{~d}, J=7.5 \mathrm{~Hz}, 2 \mathrm{H}), 7.43(\mathrm{t}, J=7.3 \mathrm{~Hz}, 2 \mathrm{H}), 7.37(\mathrm{t}, J$ $=7.2 \mathrm{~Hz}, 1 \mathrm{H}), 7.30(\mathrm{~d}, J=7.2 \mathrm{~Hz}, 2 \mathrm{H}), 6.97(\mathrm{~d}, J=8.5 \mathrm{~Hz}, 2 \mathrm{H}), 5.09(\mathrm{~s}, 2 \mathrm{H}), 4.26(\mathrm{~s}$, 2H). ${ }^{19} \mathrm{~F} \mathrm{NMR}\left(471 \mathrm{MHz}, \mathrm{CDCl}_{3}\right) \delta-34.4$ (s, 3F). ${ }^{13} \mathrm{C} \mathrm{NMR}\left(126 \mathrm{MHz}, \mathrm{CDCl}_{3}\right) \delta$ 158.4, 136.8, 130.3, 128.7, 128.1, 128.1, 127.5, 123.0 (q, $J=331.4$ Hz), 115.2, 70.1, 28.8 (q, $J=1.3 \mathrm{~Hz})$. IR (KBr): 3059, 3015, 2947, 2928, 2916, 2872, 1607, 1582, 1513 , 1466, 1455, 1439, 1386, 1299, 1244, 1179, 1140, 1105, 1012, 917, 866, 843, 817, 740, 728, 696, 623, $609 \mathrm{~cm}^{-1}$. HRMS-ESI $(\mathrm{m} / \mathrm{z})$ calcd. for $\mathrm{C}_{15} \mathrm{H}_{14} \mathrm{~F}_{3} \mathrm{OSe}\left([\mathrm{M}+\mathrm{H}]^{+}\right)$: 347.0156; found: 347.0157 .<smiles>COc1ccc(C[Se]C(F)(F)F)cc1OC</smiles>

(3,4-Dimethoxybenzyl)(trifluoromethyl)selane (3j). Light yellow liquid (37.3 mg, $63 \%$ yield), petroleum ether as eluent for column chromatography. ${ }^{1} \mathrm{H}$ NMR (500 $\left.\mathrm{MHz}, \mathrm{CDCl}_{3}\right) \delta 6.89(\mathrm{dd}, J=8.2,1.5 \mathrm{~Hz}, 1 \mathrm{H}), 6.85(\mathrm{~m}, 1 \mathrm{H}), 6.80(\mathrm{~d}, J=8.2 \mathrm{~Hz}, 1 \mathrm{H})$, $4.23(\mathrm{~s}, 2 \mathrm{H}), 3.88(\mathrm{~s}, 3 \mathrm{H}), 3.87(\mathrm{~s}, 3 \mathrm{H}) .{ }^{19} \mathrm{~F} \mathrm{NMR}\left(471 \mathrm{MHz}, \mathrm{CDCl}_{3}\right) \delta-34.4(\mathrm{~s}, 3 \mathrm{~F})$. ${ }^{13} \mathrm{C}$ NMR $\left(126 \mathrm{MHz}, \mathrm{CDCl}_{3}\right) \delta 149.3,148.8,128.3,122.9$ (q, $\left.J=331.4 \mathrm{~Hz}\right), 121.4$, 112.2, 111.4, 55.9, 29.3. IR (KBr): 3003, 2958, 2939, 2911, 2837, 1606, 1593, 1517, 
1466, 1443, 1421, 1338, 1266, 1246, 1213, 1191, 1097, 1072, 1028, 943, 875, 808, 766, 738, 720, $654 \mathrm{~cm}^{-1}$. HRMS-ESI $(\mathrm{m} / \mathrm{z})$ calcd. for $\mathrm{C}_{10} \mathrm{H}_{12} \mathrm{~F}_{3} \mathrm{O}_{2} \mathrm{Se}\left([\mathrm{M}+\mathrm{H}]^{+}\right)$: 300.9949; found: 300.9953 .<smiles>COc1cc(C[Se]C(F)(F)F)cc(OC)c1</smiles>

(3,5-Dimethoxybenzyl)(trifluoromethyl)selane (3k). ${ }^{4 \mathrm{~b}}$ Light yellow liquid (40.2 $\mathrm{mg}$, $67 \%$ yield), petroleum ether as eluent for column chromatography. ${ }^{1} \mathrm{H}$ NMR (500 $\left.\mathrm{MHz}, \mathrm{CDCl}_{3}\right) \delta 6.49(\mathrm{~d}, J=1.7 \mathrm{~Hz}, 2 \mathrm{H}), 6.38(\mathrm{~m}, 1 \mathrm{H}), 4.18(\mathrm{~s}, 2 \mathrm{H}), 3.79(\mathrm{~s}, 6 \mathrm{H}) .{ }^{19} \mathrm{~F}$ $\operatorname{NMR}\left(471 \mathrm{MHz}, \mathrm{CDCl}_{3}\right) \delta-34.5$ (s, 3F). ${ }^{13} \mathrm{C} \mathrm{NMR}\left(126 \mathrm{MHz}, \mathrm{CDCl}_{3}\right) \delta$ 161.1, 138.2, $122.9(\mathrm{q}, J=331.8 \mathrm{~Hz}), 107.0,99.8,55.4,29.3$.<smiles>FC(F)(F)[Se]Cc1ccc2c(c1)OCO2</smiles>

5-(((Trifluoromethyl)selanyl)methyl)benzo[d][1,3]dioxole (31). Light yellow liquid (35.4 mg, 63\% yield), petroleum ether as eluent for column chromatography. ${ }^{1} \mathrm{H}$ NMR $\left(500 \mathrm{MHz}, \mathrm{CDCl}_{3}\right) \delta 6.83(\mathrm{~s}, 1 \mathrm{H}), 6.80(\mathrm{~d}, J=8.2 \mathrm{~Hz}, 1 \mathrm{H}), 6.75(\mathrm{~d}, J=7.9 \mathrm{~Hz}, 1 \mathrm{H})$, $5.97(\mathrm{~s}, 2 \mathrm{H}), 4.19$ (s, 2H). ${ }^{19} \mathrm{~F} \mathrm{NMR}\left(471 \mathrm{MHz}, \mathrm{CDCl}_{3}\right) \delta-34.4(\mathrm{~s}, 3 \mathrm{~F}) .{ }^{13} \mathrm{C}$ NMR $(126$ $\left.\mathrm{MHz}, \mathrm{CDCl}_{3}\right) \delta 148.0,147.3,129.6,122.9(\mathrm{q}, J=331.2 \mathrm{~Hz}), 122.4,109.3,108.5$, 101.3, 29.3 (q, $J=1.4 \mathrm{~Hz}$ ). IR (KBr): 2955, 2925, 2855, 1634, 1601, 1504, 1490, 1446, 1360, 1252, 1212, 1189, 1099, 1073, 1041, 943, 928, 864, 811, 738, $649 \mathrm{~cm}^{-1}$. HRMS-ESI (m/z) calcd. for $\mathrm{C}_{9} \mathrm{H}_{8} \mathrm{~F}_{3} \mathrm{O}_{2} \mathrm{Se}\left([\mathrm{M}+\mathrm{H}]^{+}\right)$: 284.9636 ; found: 284.9643 .<smiles></smiles>

5-(((Trifluoromethyl)selanyl)methyl)-2,3-dihydrobenzofuran (3m). White solid (43.3 $\mathrm{mg}, 77 \%$ yield), a mixture of petroleum ether/ethyl acetate $=20: 1(\mathrm{v} / \mathrm{v})$ as eluents for column chromatography. M.p.: 40-42 ${ }^{\circ} \mathrm{C} .{ }^{1} \mathrm{H}$ NMR $\left(500 \mathrm{MHz}, \mathrm{CDCl}_{3}\right) \delta 7.18(\mathrm{~s}, 1 \mathrm{H})$, $7.08(\mathrm{~d}, J=8.1 \mathrm{~Hz}, 1 \mathrm{H}), 6.73(\mathrm{~d}, J=8.2 \mathrm{~Hz}, 1 \mathrm{H}), 4.58(\mathrm{t}, J=8.7 \mathrm{~Hz}, 2 \mathrm{H}), 4.23$ (s, 2H), $3.20(\mathrm{t}, J=8.7 \mathrm{~Hz}, 2 \mathrm{H}) .{ }^{19} \mathrm{~F}$ NMR $\left(471 \mathrm{MHz}, \mathrm{CDCl}_{3}\right) \delta-34.4(\mathrm{~s}, 3 \mathrm{~F}) .{ }^{13} \mathrm{C} \mathrm{NMR}$ $\left(126 \mathrm{MHz}, \mathrm{CDCl}_{3}\right) \delta 159.9,129.0,127.9,127.6,125.7,123.0(\mathrm{q}, J=331.2 \mathrm{~Hz}), 109.5$, 71.5, 29.6, 29.3 (q, $J=1.7 \mathrm{~Hz}$ ). IR (KBr): 2989, 2956, 292, 2860, 1616, 1598, 1491, 1444, 1434, 1368, 1322, 1292, 1253, 1206, 1191, 1147, 1121, 1094, 978, 941, 925, 
900, 828, 737, 721, $640 \mathrm{~cm}^{-1}$. HRMS-ESI $(\mathrm{m} / \mathrm{z})$ calcd. for $\mathrm{C}_{10} \mathrm{H}_{10} \mathrm{~F}_{3} \mathrm{OSe}\left([\mathrm{M}+\mathrm{H}]^{+}\right)$: 282.9843; found: 282.9842 .<smiles>COc1ccc(C[Ge]C(F)(F)F)cc1F</smiles>

(3-Fluoro-4-methoxybenzyl)(trifluoromethyl)selane (3n). Light yellow liquid (50.1 mg, $87 \%$ yield), petroleum ether as eluent for column chromatography. ${ }^{1} \mathrm{H}$ NMR (500 $\left.\mathrm{MHz}, \mathrm{CDCl}_{3}\right) \delta 7.08(\mathrm{dm}, J=11.8 \mathrm{~Hz}, 1 \mathrm{H}), 7.05(\mathrm{~d}, J=8.8 \mathrm{~Hz}, 1 \mathrm{H}), 6.90$ (t, $J=8.6$ $\mathrm{Hz}, 1 \mathrm{H}), 4.18$ (s, 2H), 3.89 (s, 3H). ${ }^{19} \mathrm{~F} \mathrm{NMR}\left(471 \mathrm{MHz}, \mathrm{CDCl}_{3}\right) \delta-34.3$ (s, 3F), 134.2 (m, 1F). ${ }^{13} \mathrm{C}$ NMR $\left(126 \mathrm{MHz}, \mathrm{CDCl}_{3}\right) \delta 151.7(\mathrm{~d}, J=246.7 \mathrm{~Hz}), 147.3(\mathrm{~d}, J=10.4$ $\mathrm{Hz}), 128.9$ (d, $J=6.4 \mathrm{~Hz}), 124.9(\mathrm{~d}, J=3.5 \mathrm{~Hz}), 122.8(\mathrm{q}, J=331.4 \mathrm{~Hz}), 116.8$ (d, $J=$ $19.0 \mathrm{~Hz}), 113.5$ (d, $J=1.6 \mathrm{~Hz}), 56.3,28.3(\mathrm{~m}) . \mathrm{IR}(\mathrm{KBr}): 3010,2962,2938,2844$, 1622, 1584, 1519, 1465, 1444, 1383, 1318, 1278, 1229, 1212, 1185, 1120, 1097, 1073, $1028,955,876,810,762,738,649 \mathrm{~cm}^{-1}$. HRMS-ESI (m/z) calcd. for $\mathrm{C}_{9} \mathrm{H}_{9} \mathrm{~F}_{4} \mathrm{OSe}([\mathrm{M}$ $\left.+\mathrm{H}]^{+}\right): 288.9749$; found: 288.9747 .<smiles>COc1ccc(C[Se]C(F)(F)F)cc1Br</smiles>

(3-Bromo-4-methoxybenzyl)(trifluoromethyl)selane (3o). Light yellow liquid (59.2 $\mathrm{mg}, 85 \%$ yield), petroleum ether as eluent for column chromatography. ${ }^{1} \mathrm{H}$ NMR (500 $\left.\mathrm{MHz}, \mathrm{CDCl}_{3}\right) \delta 7.53(\mathrm{~d}, J=1.8 \mathrm{~Hz}, 1 \mathrm{H}), 7.25(\mathrm{dd}, J=8.4,1.8 \mathrm{~Hz}, 1 \mathrm{H}), 6.85(\mathrm{~d}, J=$ $8.4 \mathrm{~Hz}, 1 \mathrm{H}), 4.18(\mathrm{~s}, 2 \mathrm{H}), 3.89$ (s, 3H). ${ }^{19} \mathrm{~F} \mathrm{NMR}\left(471 \mathrm{MHz}, \mathrm{CDCl}_{3}\right) \delta-34.3(\mathrm{~s}, 3 \mathrm{~F})$. ${ }^{13} \mathrm{C} \mathrm{NMR}\left(126 \mathrm{MHz}, \mathrm{CDCl}_{3}\right) \delta 155.5,133.8,129.6,129.2,122.8$ (q, $\left.J=331.2 \mathrm{~Hz}\right)$, 112.0, 111.9, 56.3, 27.9 (q, $J=1.3 \mathrm{~Hz})$. IR (KBr): 3010, 2945, 2840, 1602, 1571, 1498, 1463, 1441, 1405, 1298, 1281, 1261, 1220, 1199, 1184, 1097, 1072, 1055, 1021, 888, 812, 748, 738, 717, 673, $628 \mathrm{~cm}^{-1}$. HRMS-ESI (m/z) calcd. for $\mathrm{C}_{9} \mathrm{H}_{9} \mathrm{BrF}_{3} \mathrm{OSe}$ $\left([\mathrm{M}+\mathrm{H}]^{+}\right): 348.8949$; found: 348.8952 .<smiles>FC(F)(F)[Se]Cc1cc(Cl)ccc1Oc1ccccc1</smiles>

(5-Chloro-2-phenoxybenzyl)(trifluoromethyl)selane (3p). Colorless liquid (29.3 mg, $40 \%$ yield; $35.1 \mathrm{mg}, 48 \%$ yield; $40.2 \mathrm{mg}$, $55 \%$ yield), petroleum ether as eluent for column chromatography. ${ }^{1} \mathrm{H}$ NMR $\left(500 \mathrm{MHz} \mathrm{CDCl}_{3}\right) \delta$ 7.39-7.35 (m, 3H), 7.19-7.15 
$(\mathrm{m}, 2 \mathrm{H}), 7.01(\mathrm{~d}, J=8.1 \mathrm{~Hz}, 2 \mathrm{H}), 6.75(\mathrm{~d}, J=8.7 \mathrm{~Hz}, 1 \mathrm{H}), 4.20(\mathrm{~s}, 2 \mathrm{H}) .{ }^{19} \mathrm{~F}$ NMR $\left(471 \mathrm{MHz}, \mathrm{CDCl}_{3}\right) \delta-34.4$ (s, 3F). ${ }^{13} \mathrm{C} \mathrm{NMR}\left(126 \mathrm{MHz}, \mathrm{CDCl}_{3}\right) \delta 156.3,154.0,130.3$, $130.0,129.7,129.1,128.2,124.2,122.9$ (q, $J=331.6 \mathrm{~Hz}), 119.1,119.1,23.0$ (q, $J=$ $1.3 \mathrm{~Hz}$ ). IR (KBr): 3064, 3041, 2958, 2927, 2852, 1590, 1580, 1481, 1422, 1405, 1289, 1245, 1217, 1204, 1181, 1098, 1073, 1023, 903, 884, 855, 817, 792, 739, 692 $\mathrm{cm}^{-1}$. HRMS-ESI $(\mathrm{m} / \mathrm{z})$ calcd. for $\mathrm{C}_{14} \mathrm{H}_{11} \mathrm{ClF}_{3} \mathrm{OSe}\left([\mathrm{M}+\mathrm{H}]^{+}\right)$: 366.9610; found: 366.9610 .<smiles>COc1ccc([N+](=O)[O-])cc1C[Se]C(F)(F)F</smiles>

(2-Methoxy-5-nitrobenzyl)(trifluoromethyl)selane (3q). White solid (25.6 mg, 41\% yield), a mixture of petroleum ether/ethyl acetate $=20: 1(\mathrm{v} / \mathrm{v})$ as eluents for column chromatography. M.p.: 76-78 ${ }^{\circ} \mathrm{C} .{ }^{1} \mathrm{H}$ NMR $\left(500 \mathrm{MHz}, \mathrm{CDCl}_{3}\right) \delta 8.21(\mathrm{dd}, J=9.0,2.8$ $\mathrm{Hz}, 1 \mathrm{H}), 8.17(\mathrm{~d}, J=2.7 \mathrm{~Hz}, 1 \mathrm{H}), 6.95(\mathrm{~d}, J=9.0 \mathrm{~Hz}, 1 \mathrm{H}), 4.18(\mathrm{~s}, 2 \mathrm{H}), 3.99$ (s, 3H). ${ }^{19} \mathrm{~F}$ NMR $\left(471 \mathrm{MHz}, \mathrm{CDCl}_{3}\right) \delta-34.5(\mathrm{~s}, 3 \mathrm{~F}) .{ }^{13} \mathrm{C} \mathrm{NMR}\left(126 \mathrm{MHz}, \mathrm{CDCl}_{3}\right) \delta 162.0$, $141.2,127.1,125.6,125.5,122.8(\mathrm{q}, J=331.0 \mathrm{~Hz}), 110.3,56.4,23.0(\mathrm{q}, J=1.8 \mathrm{~Hz})$. IR (KBr): 3083, 3028, 2952, 2922, 2850, 1646, 1613, 1593, 1501, 1470, 1425, 1361, 1343, 1282, 1216, 1126, 1104, 1023, 936, 908, 821, 814, 750, 737, 730, $636 \mathrm{~cm}^{-1}$. HRMS-ESI $(\mathrm{m} / \mathrm{z})$ calcd. for $\mathrm{C}_{9} \mathrm{H}_{8} \mathrm{~F}_{3} \mathrm{NNaO}_{3} \mathrm{Se}\left([\mathrm{M}+\mathrm{Na}]^{+}\right)$: 337.9514; found: 337.9512 .<smiles>COc1ccc(OC(F)(F)F)cc1COC(F)(F)F</smiles>

(2-Methoxy-5-(trifluoromethoxy)benzyl)(trifluoromethyl)selane (3r). Light yellow liquid (28.0 mg, 40\% yield), petroleum ether as eluent for column chromatography. ${ }^{1} \mathrm{H}$ NMR $\left(500 \mathrm{MHz}, \mathrm{CDCl}_{3}\right) \delta$ 7.14-7.12 (m, 2H), $6.85(\mathrm{~d}, J=9.7 \mathrm{~Hz}, 1 \mathrm{H}), 4.13$ (s, 2H), $3.88(\mathrm{~s}, 3 \mathrm{H}) .{ }^{19} \mathrm{~F}$ NMR (471 MHz, $\left.\mathrm{CDCl}_{3}\right) \delta-34.7$ (s, 3F), -58.5 (s, 3F). ${ }^{13} \mathrm{C}$ NMR (126 MHz, $\left.\mathrm{CDCl}_{3}\right) \delta 155.7,142.2(\mathrm{q}, J=1.9 \mathrm{~Hz}), 127.3,123.1,123.0$ (q, $J=$ $331.6 \mathrm{~Hz}), 121.8,120.5$ (q, $J=256.9 \mathrm{~Hz}), 111.1,55.9,23.4$ (q, $J=1.8 \mathrm{~Hz})$. IR (KBr): 3014, 2950, 2845, 1612, 1501, 1466, 1445, 1434, 1258, 1233, 1216, 1182, 1170, 1119, 
1097, 1074, 1032, 980, 894, 876, 816, 738, 691, $608 \mathrm{~cm}^{-1}$. HRMS-EI (m/z) calcd for $\mathrm{C}_{10} \mathrm{H}_{8} \mathrm{~F}_{6} \mathrm{O}_{2}{ }^{74} \mathrm{Se}([\mathrm{M}]): 347.9653$; found: 347.9648 .<smiles>Cc1ccc(C)c(C[Ge]C(F)(F)F)c1</smiles>

(2,5-Dimethylbenzyl)(trifluoromethyl)selane (3s). Light yellow liquid (17.6 mg, 33\% yield), petroleum ether as eluent for column chromatography. ${ }^{1} \mathrm{H}$ NMR $(500 \mathrm{MHz}$, $\left.\mathrm{CDCl}_{3}\right) \delta 7.09(\mathrm{~s}, 1 \mathrm{H}), 7.07(\mathrm{~d}, J=7.7 \mathrm{~Hz}, 1 \mathrm{H}), 7.01(\mathrm{~d}, J=7.7 \mathrm{~Hz}, 1 \mathrm{H}), 4.25(\mathrm{~s}, 2 \mathrm{H})$, $2.36(\mathrm{~s}, 3 \mathrm{H}), 2.31(\mathrm{~s}, 3 \mathrm{H}) .{ }^{19} \mathrm{~F}$ NMR $\left(471 \mathrm{MHz}, \mathrm{CDCl}_{3}\right) \delta-34.6(\mathrm{~s}, 3 \mathrm{~F}) .{ }^{13} \mathrm{C}$ NMR $(126$ $\left.\mathrm{MHz}, \mathrm{CDCl}_{3}\right) \delta 136.0,133.5,133.1,130.7,130.7,129.0,122.9$ (q, $J=331.6 \mathrm{~Hz}$ ), $27.3(\mathrm{q}, J=1.1 \mathrm{~Hz}), 20.8,18.5$. IR (KBr): 3048, 3003, 2925, 2860, 1618, 1505, 1445, 1381, 1291, 1202, 1099, 1074, 993, 931, 884, 813, 738, $716 \mathrm{~cm}^{-1}$. HRMS-ESI (m/z) calcd. for $\mathrm{C}_{10} \mathrm{H}_{12} \mathrm{~F}_{3} \mathrm{Se}\left([\mathrm{M}+\mathrm{H}]^{+}\right)$: 268.0092; found: 268.0096 .<smiles>FC(F)(F)[GeH2]Cc1ccc2ccccc2c1</smiles>

(Naphthalen-2-ylmethyl)(trifluoromethyl)selane (3t). ${ }^{4 \mathrm{~b}}$ White solid $(47.0 \mathrm{mg}, 81 \%$ yield), petroleum ether as eluent for column chromatography. M.p.: $53-55{ }^{\circ} \mathrm{C} .{ }^{1} \mathrm{H}$ NMR (500 MHz, $\left.\mathrm{CDCl}_{3}\right) \delta$ 7.84-7.79 (m, 4H), 7.52-7.49 (m, 2H), 7.47 (dd, $J=8.3$, $1.3 \mathrm{~Hz}, 1 \mathrm{H}), 4.43(\mathrm{~s}, 2 \mathrm{H}) .{ }^{19} \mathrm{~F} \mathrm{NMR}\left(471 \mathrm{MHz}, \mathrm{CDCl}_{3}\right) \delta-34.3$ (s, 3F). ${ }^{13} \mathrm{C}$ NMR (126 $\left.\mathrm{MHz}, \mathrm{CDCl}_{3}\right) \delta 133.4,133.4,132.8,128.8,127.8,127.7,127.7,126.8,126.5,126.3$, $122.9(\mathrm{q}, J=331.9 \mathrm{~Hz}), 29.5(\mathrm{q}, J=1.2 \mathrm{~Hz})$.<smiles>FC(F)(F)[Se]COc1ccc2ccccc2c1</smiles>

((Naphthalen-2-yloxy)methyl)(trifluoromethyl)selane (3u). Colorless liquid (39.7 mg, $65 \%$ yield), petroleum ether as eluent for column chromatography. ${ }^{1} \mathrm{H}$ NMR (500 $\left.\mathrm{MHz}, \mathrm{CDCl}_{3}\right) \delta 7.82(\mathrm{~d}, J=8.8 \mathrm{~Hz}, 2 \mathrm{H}), 7.78(\mathrm{~d}, J=8.2 \mathrm{~Hz}, 1 \mathrm{H}), 7.50(\mathrm{t}, J=7.1 \mathrm{~Hz}$, $1 \mathrm{H}), 7.43(\mathrm{t}, J=7.3 \mathrm{~Hz}, 1 \mathrm{H}), 7.20(\mathrm{dd}, J=8.9,2.5 \mathrm{~Hz}, 1 \mathrm{H}), 7.17(\mathrm{~d}, J=2.3 \mathrm{~Hz}, 1 \mathrm{H})$, $5.93(\mathrm{~s}, 2 \mathrm{H}) .{ }^{19} \mathrm{~F}$ NMR $\left(471 \mathrm{MHz}, \mathrm{CDCl}_{3}\right) \delta-33.2(\mathrm{~s}, 3 \mathrm{~F}) .{ }^{13} \mathrm{C} \mathrm{NMR}(126 \mathrm{MHz}$, $\left.\mathrm{CDCl}_{3}\right) \delta 153.9,134.0,130.1,130.0,127.8,127.1,126.8,124.7,122.5$ (q, $J=331.9$ Hz), 119.0, 109.4, 64.9 (q, $J=2.1 \mathrm{~Hz})$. IR (KBr): 3060, 3027, 2928, 2855, 1631, 1600, 
1511, 1468, 1436, 1391, 1359, 1294, 1252, 1209, 1171, 1125, 1097, 1036, 1020, 958, 875, 836, 811, 770, 747, 739, 694, $622 \mathrm{~cm}^{-1}$. HRMS-EI (m/z) calcd for $\mathrm{C}_{12} \mathrm{H}_{9} \mathrm{~F}_{3} \mathrm{OSe}$ ([M]): 305.9771; found: 305.9758.<smiles>FC(F)(F)OCSc1cccc2cccc(Cl)c12</smiles>

(8-Chloronaphthalen-1-yl)(((trifluoromethyl)selanyl)methyl)sulfane $\quad$ (3v). Light yellow liquid (22.4 mg, 31\% yield), petroleum ether as eluent for column chromatography. ${ }^{1} \mathrm{H}$ NMR $\left(500 \mathrm{MHz}, \mathrm{CDCl}_{3}\right) \delta$ 7.80-7.77 (m, 2H), $7.64(\mathrm{~d}, J=7.3$ $\mathrm{Hz}, 1 \mathrm{H}), 7.62(\mathrm{dd}, J=7.8,1.0 \mathrm{~Hz}, 1 \mathrm{H}), 7.45$ (t, $J=7.8 \mathrm{~Hz}, 1 \mathrm{H}), 7.38$ (t, $J=7.8 \mathrm{~Hz}$, 1H), $4.48(\mathrm{~s}, 2 \mathrm{H}) .{ }^{19} \mathrm{~F}$ NMR $\left(471 \mathrm{MHz}, \mathrm{CDCl}_{3}\right) \delta-34.3(\mathrm{~s}, 3 \mathrm{~F}) .{ }^{13} \mathrm{C} \mathrm{NMR}(126 \mathrm{MHz}$, $\left.\mathrm{CDCl}_{3}\right) \delta 136.9,131.8,131.6,130.8,130.4,129.9,129.4,128.9,126.1,125.9,122.6$ $(\mathrm{q}, J=332.1 \mathrm{~Hz}), 30.3 . \mathrm{IR}(\mathrm{KBr}): 3059,2921,2851,1648,1597,1552,1497,1423$, 1361, 1318, 1198, 1175, 1124, 1094, 1075, 980, 883, 810, 754, 738, 726, $673 \mathrm{~cm}^{-1}$. HRMS-EI (m/z) calcd for $\mathrm{C}_{12} \mathrm{H}_{8} \mathrm{ClF}_{3} \mathrm{SSe}([\mathrm{M}])$ : 355.9131 ; found: 355.9137 .<smiles>FC(F)(F)[As]c1ccc2ccc3cccc4ccc1c2c34</smiles>

(Pyren-1-ylmethyl)(trifluoromethyl)selane (3w). Light yellow solid (30.2 mg, 39\% yield), a mixture of petroleum ether/ethyl acetate $=15: 1(\mathrm{v} / \mathrm{v})$ as eluents for column chromatography. M.p.: $85-87{ }^{\circ} \mathrm{C} .{ }^{1} \mathrm{H}$ NMR $\left(500 \mathrm{MHz}, \mathrm{CDCl}_{3}\right) \delta 8.26(\mathrm{~d}, J=9.3 \mathrm{~Hz}$, $1 \mathrm{H}), 8.20-8.18(\mathrm{~m}, 2 \mathrm{H}), 8.15-8.12(\mathrm{~m}, 2 \mathrm{H}), 8.05(\mathrm{~m}, 2 \mathrm{H}), 8.01(\mathrm{t}, J=7.6 \mathrm{~Hz}, 1 \mathrm{H})$, $7.87(\mathrm{~d}, J=7.7 \mathrm{~Hz}, 1 \mathrm{H}), 3.48(\mathrm{t}, J=7.4 \mathrm{~Hz}, 2 \mathrm{H}), 3.10(\mathrm{t}, J=7.2 \mathrm{~Hz}, 2 \mathrm{H}), 2.37(\mathrm{~m}$, 2H). $\left.{ }^{19} \mathrm{~F} \mathrm{NMR} \mathrm{(471} \mathrm{MHz,} \mathrm{CDCl}_{3}\right) \delta-33.9$ (s, 3F). ${ }^{13} \mathrm{C} \mathrm{NMR}\left(126 \mathrm{MHz}, \mathrm{CDCl}_{3}\right) \delta$ $134.7,131.4,130.9,130.2$, 128.7, 127.6, 127.5, 127.3, 126.9, 126.0, 125.2, 125.1, 125.0, 124.9, 124.9, 123.0, 122.7 (q, $J=330.9 \mathrm{~Hz}), 33.1,32.0,25.5$. IR (KBr): 3040, 2928, 2880, 1602, 1586, 1458, 1430, 1414, 1277, 1236, 1198, 1184, 1141, 1126, 1115, 1093, 1018, 967, 842, 756, 734, 710, 678, $637 \mathrm{~cm}^{-1}$. HRMS-ESI (m/z) calcd. for $\mathrm{C}_{20} \mathrm{H}_{16} \mathrm{~F}_{3} \mathrm{Se}\left([\mathrm{M}+\mathrm{H}]^{+}\right): 393.0364$; found: 393.0364 .<smiles></smiles> 
(7-Phenylheptyl)(trifluoromethyl)selane (3x). Colorless liquid (38.1 mg, 59\% yield), petroleum ether as eluent for column chromatography. ${ }^{1} \mathrm{H}$ NMR $\left(500 \mathrm{MHz}, \mathrm{CDCl}_{3}\right) \delta$ $7.29(\mathrm{t}, J=7.6 \mathrm{~Hz}, 2 \mathrm{H}), 7.20-7.18(\mathrm{~m}, 3 \mathrm{H}), 2.98(\mathrm{t}, J=7.4 \mathrm{~Hz}, 2 \mathrm{H}), 2.62(\mathrm{t}, J=7.6$ $\mathrm{Hz}, 2 \mathrm{H}), 1.79$ (m, 2H), $1.64(\mathrm{~m}, 2 \mathrm{H}), 1,41-1.36(\mathrm{~m}, 6 \mathrm{H}) .{ }^{19} \mathrm{~F}$ NMR (471 MHz, $\mathrm{CDCl}_{3}$ ) $\delta$-34.1(s, 3F). ${ }^{13} \mathrm{C}$ NMR $\left(126 \mathrm{MHz}, \mathrm{CDCl}_{3}\right) \delta 142.7,128.4,128.3,125.7,122.7$ (q, $J$ $=330.2 \mathrm{~Hz}), 35.9,31.4,30.2,29.5,29.1,28.8,25.8$ (q, $J=1.3 \mathrm{~Hz}) . \mathrm{IR}(\mathrm{KBr}): 3085$, 3063, 3027, 2930, 2856, 1604, 1496, 1464, 1454, 1249, 1205, 1100, 1076, 1030, 746, 738, $698 \mathrm{~cm}^{-1}$. HRMS-EI (m/z) calcd for $\mathrm{C}_{14} \mathrm{H}_{19} \mathrm{~F}_{3} \mathrm{Se}([\mathrm{M}])$ : 324.0604; found: 324.0592 .

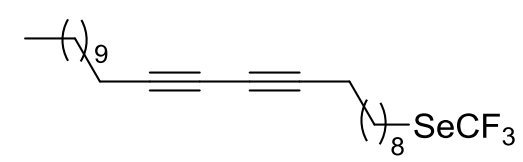

Tetracosa-9,11-diyn-1-yl(trifluoromethyl)selane (3y). Colorless liquid (43.3 mg, 45\% yield), petroleum ether as eluent for column chromatography. ${ }^{1} \mathrm{H}$ NMR $(500 \mathrm{MHz}$, $\left.\mathrm{CDCl}_{3}\right) \delta 2.98(\mathrm{t}, J=7.5 \mathrm{~Hz}, 2 \mathrm{H}), 2.26-2.23(\mathrm{~m}, 4 \mathrm{H}), 1.78(\mathrm{~m}, 2 \mathrm{H}), 1.54-1.49(\mathrm{~m}, 4 \mathrm{H})$, 1.41-1.36 (m, 6H), 1.32-1.26 (m, 20H), $0.88(\mathrm{t}, J=7.1 \mathrm{~Hz}, 3 \mathrm{H}) .{ }^{19} \mathrm{~F}$ NMR $(471 \mathrm{MHz}$, $\left.\mathrm{CDCl}_{3}\right) \delta-34.1(\mathrm{~s}, 3 \mathrm{~F}) .{ }^{13} \mathrm{C} \mathrm{NMR}\left(126 \mathrm{MHz}, \mathrm{CDCl}_{3}\right) \delta 122.7(\mathrm{q}, J=330.4 \mathrm{~Hz}), 77.7$, 77.4, 65.4, 65.2, 31.9, 30.2, 29.7, 29.6, 29.6, 29.5, 29.4, 29.1, 28.9, 28.9, 28.8, 28.7, 28.4, 28.3, 25.8, 22.7, 19.2, 19.2, 14.1. IR (KBr): 2926, 2854, 1466, 1427, 1370, 1351, 1323, 1301, 1214, 1139, 1101, 1076, 1020, 799, 738, 722, $644 \mathrm{~cm}^{-1}$. HRMS-EI (m/z) calcd for $\mathrm{C}_{25} \mathrm{H}_{41} \mathrm{~F}_{3}{ }^{74} \mathrm{Se}([\mathrm{M}])$ : 472.2385 ; found: 472.2391 .

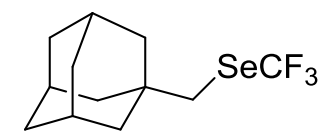

(((3r,5r,7r)-Adamantan-1-yl)methyl)(trifluoromethyl)selane (3z). Colorless liquid (26.9 mg, 45\% yield), petroleum ether as eluent for column chromatography. ${ }^{1} \mathrm{H}$ NMR $\left(500 \mathrm{MHz}, \mathrm{CDCl}_{3}\right) \delta 2.83(\mathrm{~s}, 2 \mathrm{H}), 2.01(\mathrm{~s}, 3 \mathrm{H}), 1.72-1.61(\mathrm{~m}, 6 \mathrm{H}), 1.55(\mathrm{~d}, J=2.3 \mathrm{~Hz}$, $6 \mathrm{H}) .{ }^{19} \mathrm{~F} \mathrm{NMR}\left(471 \mathrm{MHz}, \mathrm{CDCl}_{3}\right) \delta-34.1$ (s, 3F). ${ }^{13} \mathrm{C} \mathrm{NMR}\left(126 \mathrm{MHz}, \mathrm{CDCl}_{3}\right) \delta$ 122.5 (q, $J=330.5 \mathrm{~Hz}), 42.0,41.0,36.5,32.8,28.4$. IR (KBr): 2905, 2850, 1453, 1413, 1361, 1345, 1317, 1271, 1223, 1144, 1115, 1098, 1074, 974, 940, 918, 831, 738, 654, $631 \mathrm{~cm}^{-1}$. HRMS-EI (m/z) calcd for $\mathrm{C}_{12} \mathrm{H}_{17} \mathrm{~F}_{3} \mathrm{Se}([\mathrm{M}])$ : 298.0448; found: 298.0433. 
<smiles>O=C1c2ccccc2COc2ccc(CC(F)(F)F)cc21</smiles>

2-(((Trifluoromethyl)selanyl)methyl)dibenzo[b,e]oxepin-11(6H)-one (3aa). White solid (30.9 mg, 42\% yield; $40.4 \mathrm{mg}$, 54\% yield), a mixture of petroleum ether/ethyl acetate $=15: 1(\mathrm{v} / \mathrm{v})$ as eluents for column chromatography. M.p.: 85-87 ${ }^{\circ} \mathrm{C} .{ }^{1} \mathrm{H}$ NMR $\left(500 \mathrm{MHz}, \mathrm{CDCl}_{3}\right) \delta 8.20(\mathrm{~d}, J=2.2 \mathrm{~Hz}, 1 \mathrm{H}), 7.90(\mathrm{~d}, J=7.7 \mathrm{~Hz}, 1 \mathrm{H}), 7.58(\mathrm{t}, J=7.4$ $\mathrm{Hz}, 1 \mathrm{H}), 7.50-7.47$ (m, 2H), 7.37 (d, $J=7.4 \mathrm{~Hz}, 1 \mathrm{H}), 7.04$ (d, $J=8.5 \mathrm{~Hz}, 1 \mathrm{H}), 5.20$ (s, 2H), 4.27 (s, 2H). ${ }^{19} \mathrm{~F}$ NMR (471 MHz, $\left.\mathrm{CDCl}_{3}\right) \delta-34.3$ (s, 3F). ${ }^{13} \mathrm{C}$ NMR (126 MHz, $\left.\mathrm{CDCl}_{3}\right) \delta 190.6,160.8,140.3,135.8,135.4,132.9,132.3,129.9,129.5,129.4,127.9$, 125.3, 122.8 (q, $J=331.5 \mathrm{~Hz}), 121.6,73.7,28.3$ (q, $J=1.4 \mathrm{~Hz}) . \mathrm{IR}(\mathrm{KBr}): 2924,2854$, 1640, 1608, 1596, 1492, 1458, 1408, 1381, 1304, 1287, 1240, 1220, 1206, 1199, 1166, 1153, 1142, 1128, 1096, 1073, 1018, 1000, 980, 927, 861, 835, 761, 737, 707, 654 $\mathrm{cm}^{-1}$. HRMS-ESI $(\mathrm{m} / \mathrm{z})$ calcd. for $\mathrm{C}_{16} \mathrm{H}_{12} \mathrm{~F}_{3} \mathrm{O}_{2} \mathrm{Se}\left([\mathrm{M}+\mathrm{H}]^{+}\right)$: 372.9949; found: 372.9946.<smiles>Nc1c(CC(F)(F)F)cccc1C(=O)c1ccc(Br)cc1</smiles>

(2-Amino-3-(((trifluoromethyl)selanyl)methyl)phenyl)(4-bromophenyl)methanone

(3ab). Yellow solid (62.7 mg, 72\% yield), a mixture of petroleum ether/ethyl acetate $=$ 20:1 (v/v) as eluents for column chromatography. M.p.: 121-123 ${ }^{\circ} \mathrm{C} .{ }^{1} \mathrm{H}$ NMR (500 $\left.\mathrm{MHz}, \mathrm{CDCl}_{3}\right) \delta 7.60(\mathrm{~d}, J=8.2 \mathrm{~Hz}, 2 \mathrm{H}), 751(\mathrm{~d}, J=8.2 \mathrm{~Hz}, 2 \mathrm{H}), 7.41(\mathrm{~d}, J=8.0 \mathrm{~Hz}$, $1 \mathrm{H}), 7.36(\mathrm{~d}, J=7.3 \mathrm{~Hz}, 1 \mathrm{H}), 6.62(\mathrm{t}, J=7.8 \mathrm{~Hz}, 1 \mathrm{H}), 6.38$ (brs, 2H), $4.28(\mathrm{~s}, 2 \mathrm{H}) .{ }^{19} \mathrm{~F}$ $\operatorname{NMR}\left(471 \mathrm{MHz}, \mathrm{CDCl}_{3}\right) \delta-34.0(\mathrm{~s}, 3 \mathrm{~F}) .{ }^{13} \mathrm{C} \mathrm{NMR}\left(126 \mathrm{MHz}, \mathrm{CDCl}_{3}\right) \delta 198.0,149.0$, 138.7, 136.0, 134.9, 131.4, 130.8, 126.2, 122.6 (q, $J=331.6 \mathrm{~Hz}), 120.1,118.8,115.6$, 26.0 (q, $J=1.7 \mathrm{~Hz})$. IR (KBr): 3464, 3316, 3073, 3057, 3026, 1629, 1609, 1584, 1562 , 1555, 1482, 1444, 1420, 1315, 1286, 1245, 1210, 1189, 1167, 1144, 1098, 1069, 1011, 966, 957, 860, 843, 803, 795, 740, $651 \mathrm{~cm}^{-1}$. HRMS-ESI $(\mathrm{m} / \mathrm{z})$ calcd. for $\mathrm{C}_{15} \mathrm{H}_{12} \mathrm{BrF}_{3} \mathrm{NOSe}\left([\mathrm{M}+\mathrm{H}]^{+}\right)$: 437.9214; found: 437.9212 . 


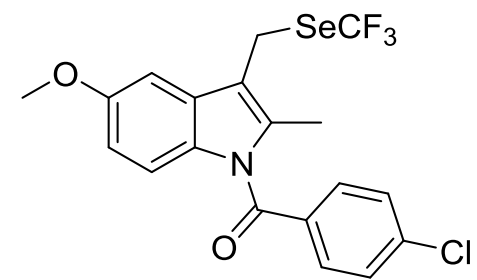

(4-Chlorophenyl)(5-methoxy-2-methyl-3-(((trifluoromethyl)selanyl)methyl)-1H-indol -1-yl)methanone (3ac). Light yellow solid (81.4 mg, 88\% yield), a mixture of petroleum ether/ethyl acetate $=20: 1(\mathrm{v} / \mathrm{v})$ as eluents for column chromatography. M.p.: 98-100 ${ }^{\circ} \mathrm{C} .{ }^{1} \mathrm{H}$ NMR $\left(500 \mathrm{MHz}, \mathrm{CDCl}_{3}\right) \delta 7.65(\mathrm{~d}, J=8.4 \mathrm{~Hz}, 2 \mathrm{H}), 7.48(\mathrm{~d}, J=8.4 \mathrm{~Hz}$, $2 \mathrm{H}), 6.98(\mathrm{~d}, J=2.2 \mathrm{~Hz}, 1 \mathrm{H}), 6.87(\mathrm{~d}, J=9.0 \mathrm{~Hz}, 1 \mathrm{H}), 6.71(\mathrm{dd}, J=9.0,2.3 \mathrm{~Hz}, 1 \mathrm{H})$, 4.39 (s, 2H), 3.86 (s, 3H), 2.40 (s, 3H). $\left.{ }^{19} \mathrm{~F} \mathrm{NMR} \mathrm{(471} \mathrm{MHz,} \mathrm{CDCl}_{3}\right) \delta-34.2$ (s, 3F). ${ }^{13} \mathrm{C}$ NMR $\left(126 \mathrm{MHz}, \mathrm{CDCl}_{3}\right) \delta 168.2,156.1,139.6,136.4,133.6,131.2,130.9,129.4$, $129.2,123.0(\mathrm{q}, J=332.0 \mathrm{~Hz}), 115.1,113.1,112.1,101.1,55.8,18.6,13.1$. IR $(\mathrm{KBr})$ : 2989, 2928, 2830, 1659, 1621, 1600, 1477, 1463, 1436, 1359, 1334, 1289, 1229, 1217 , 1206, 1177, 1140, 1097, 1068, 1039, 1011, 977, 885, 873, 844, 802, 758, 738, 695, 650, 627, 614, $604 \mathrm{~cm}^{-1}$. HRMS-ESI (m/z) calcd. for $\mathrm{C}_{19} \mathrm{H}_{16} \mathrm{ClF}_{3} \mathrm{NO}_{2} \mathrm{Se}\left([\mathrm{M}+\mathrm{H}]^{+}\right)$: 461.9981; found: 461.9978 .

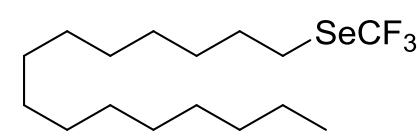

Pentadecyl(trifluoromethyl)selane (3ad). Colorless liquid (40.1 mg, 56\% yield), petroleum ether as eluent for column chromatography. ${ }^{1} \mathrm{H} \mathrm{NMR}\left(500 \mathrm{MHz}, \mathrm{CDCl}_{3}\right) \delta$ $2.98(\mathrm{t}, J=7.5 \mathrm{~Hz}, 2 \mathrm{H}), 1.79(\mathrm{~m}, 2 \mathrm{H}), 1.40(\mathrm{~m}, 2 \mathrm{H}), 1.31-1.27(\mathrm{~m}, 22 \mathrm{H}), 0.89$ (t, $J=$ $7.0 \mathrm{~Hz}, 3 \mathrm{H}) .{ }^{19} \mathrm{~F}$ NMR $\left(471 \mathrm{MHz}, \mathrm{CDCl}_{3}\right) \delta-34.2(\mathrm{~s}, 3 \mathrm{~F}) .{ }^{13} \mathrm{C} \mathrm{NMR}(126 \mathrm{MHz}$, $\left.\mathrm{CDCl}_{3}\right) \delta 122.7(\mathrm{q}, J=330.7 \mathrm{~Hz}), 31.9,30.2,29.7,29.7,29.7,29.6,29.6,29.6,29.4$, 29.4, 28.9, 25.9, 22.7, 14.0. IR (KBr): 2955, 2925, 2854, 1467, 1378, 1260, 1142, 1118, 1077, 738, $721 \mathrm{~cm}^{-1}$. HRMS-EI (m/z) calcd for $\mathrm{C}_{16} \mathrm{H}_{31} \mathrm{~F}_{3}{ }^{74} \mathrm{Se}([\mathrm{M}]): 354.1603$; found: 354.1601 .

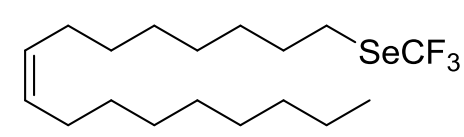

(Z)-Heptadec-8-en-1-yl(trifluoromethyl)selane (3ae). Colorless liquid (39.3 mg, 51\% yield), petroleum ether as eluent for column chromatography. ${ }^{1} \mathrm{H}$ NMR $(500 \mathrm{MHz}$, $\left.\mathrm{CDCl}_{3}\right) \delta 5.39-5.34(\mathrm{~m}, 2 \mathrm{H}), 2.98(\mathrm{t}, J=7.5 \mathrm{~Hz}, 2 \mathrm{H}), 2.02(\mathrm{~m}, 4 \mathrm{H}), 1.79(\mathrm{~m}, 2 \mathrm{H})$, 
1.32-1.27 (m, 20H), $0.89(\mathrm{t}, J=7.1 \mathrm{~Hz}, 3 \mathrm{H}) .{ }^{19} \mathrm{~F}$ NMR (471 MHz, $\left.\mathrm{CDCl}_{3}\right) \delta-34.2(\mathrm{~s}$, 3F). ${ }^{13} \mathrm{C} \mathrm{NMR}\left(126 \mathrm{MHz}, \mathrm{CDCl}_{3}\right) \delta 130.1,129.6,122.7$ (q, $\left.J=330.8 \mathrm{~Hz}\right), 31.9,30.2$, 29.8, 29.7, 29.6, 29.5, 29.3, 29.0, 28.8, 27.2, 27.1, 25.8, 22.7, 14.1. IR (KBr): 3005, 2955, 2926, 2855, 1465, 1378, 1250, 1209, 1142, 1118, 1101, 1076, 738, $722 \mathrm{~cm}^{-1}$. HRMS-EI (m/z) calcd for $\mathrm{C}_{17} \mathrm{H}_{33}{ }^{74} \mathrm{Se}\left(\left[\mathrm{M}-\mathrm{CF}_{3}\right]\right): 311.1807$; found: 311.1810 .

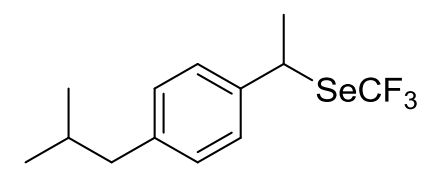

(1-(4-Isobutylphenyl)ethyl)(trifluoromethyl)selane (3af). Light yellow liquid (34.5 mg, $56 \%$ yield), petroleum ether as eluent for column chromatography. ${ }^{1} \mathrm{H}$ NMR (500 $\left.\mathrm{MHz}, \mathrm{CDCl}_{3}\right) \delta 7.30(\mathrm{~d}, J=8.0 \mathrm{~Hz}, 2 \mathrm{H}), 7.14(\mathrm{~d}, J=8.0 \mathrm{~Hz}, 2 \mathrm{H}), 4.82(\mathrm{q}, J=7.0 \mathrm{~Hz}$, $1 \mathrm{H}), 2.49(\mathrm{~d}, J=7.2 \mathrm{~Hz}, 2 \mathrm{H}), 1.95(\mathrm{~d}, J=7.0 \mathrm{~Hz}, 3 \mathrm{H}), 1.89(\mathrm{~m}, 1 \mathrm{H}), 0.93$ (d, $J=6.6$ $\mathrm{Hz}, 6 \mathrm{H}) .{ }^{19} \mathrm{~F}$ NMR $\left(471 \mathrm{MHz}, \mathrm{CDCl}_{3}\right) \delta-33.6$ (s, 3F). ${ }^{13} \mathrm{C} \mathrm{NMR}\left(126 \mathrm{MHz}, \mathrm{CDCl}_{3}\right) \delta$ 141.6, 138.3, 129.5, 126.9, 123.3 (q, $J=332.4 \mathrm{~Hz}), 45.1,41.7,30.2$, 23.2, 22.4. IR (KBr): 3027, 2957, 2926, 2870, 1635, 1511, 1451, 1421, 1384, 1368, 1279, 1170, 1100, 1056, 1040, 1020, 876, 846, 800, 737, 713, $608 \mathrm{~cm}^{-1}$. HRMS-ESI (m/z) calcd. for $\mathrm{C}_{13} \mathrm{H}_{16} \mathrm{~F}_{3} \mathrm{Se}\left([\mathrm{M}-\mathrm{H}]^{-}\right)$: 309.0375 ; found: 309.0376 .<smiles>CC([Se]C(F)(F)F)c1ccc(-c2ccccc2)c(F)c1</smiles>

(1-(2-Fluoro-[1,1'-biphenyl]-4-yl)ethyl)(trifluoromethyl)selane (3ag). Light pink liquid (40.9 mg, 59\% yield), petroleum ether as eluent for column chromatography. ${ }^{1} \mathrm{H}$ NMR (500 MHz, $\left.\mathrm{CDCl}_{3}\right) \delta 7.56(\mathrm{~d}, J=7.8 \mathrm{~Hz}, 2 \mathrm{H}), 7.48-7.38(\mathrm{~m}, 4 \mathrm{H}), 7.23(\mathrm{~d}, J$ $=8.0 \mathrm{~Hz}, 1 \mathrm{H}), 7.19(\mathrm{~d}, J=11.5 \mathrm{~Hz}, 1 \mathrm{H}), 4.81(\mathrm{q}, J=7.1 \mathrm{~Hz}, 1 \mathrm{H}), 1.95(\mathrm{~d}, J=7.1 \mathrm{~Hz}$, 3H). ${ }^{19} \mathrm{~F} \mathrm{NMR}\left(471 \mathrm{MHz}, \mathrm{CDCl}_{3}\right) \delta-33.4$ (s, 3F), 116.9 (m, 1F). ${ }^{13} \mathrm{C}$ NMR (126 MHz, $\left.\mathrm{CDCl}_{3}\right) \delta 159.7(\mathrm{~d}, J=248.4 \mathrm{~Hz}), 142.9(\mathrm{~d}, J=7.4 \mathrm{~Hz}), 135.2,131.1(\mathrm{~d}, J=3.8 \mathrm{~Hz})$, $129.0(\mathrm{~d}, J=3.0 \mathrm{~Hz}), 128.7(\mathrm{~d}, J=13.6 \mathrm{~Hz}), 128.5,127.9,123.2(\mathrm{~d}, J=3.3 \mathrm{~Hz})$, $123.1(\mathrm{q}, J=332.0 \mathrm{~Hz}), 115.0(\mathrm{~d}, J=23.9 \mathrm{~Hz}), 40.7,23.0 . \mathrm{IR}(\mathrm{KBr}): 3068,3036$, 2927, 2876, 1622, 1581, 1561, 1513, 1485, 1458, 1449, 1416, 1383, 1267, 1182, 1155, 1147, 1129, 1115, 1101, 1076, 1045, 983, 913, 872, 835, 738, 724, 701, $660 \mathrm{~cm}^{-1}$. HRMS-ESI (m/z) calcd. for $\mathrm{C}_{15} \mathrm{H}_{11} \mathrm{~F}_{4}{ }^{82} \mathrm{Se}\left([\mathrm{M}-\mathrm{H}]^{-}\right)$: 348.9969 ; found: 348.9977. 
<smiles>COc1ccc2cc(C(C)[As]C(F)(F)F)ccc2c1</smiles>

(1-(6-Methoxynaphthalen-2-yl)ethyl)(trifluoromethyl)selane (3ah). White solid (54.4 $\mathrm{mg}, 82 \%$ yield), petroleum ether as eluent for column chromatography. M.p.: $49-51^{\circ} \mathrm{C}$. ${ }^{1} \mathrm{H} \mathrm{NMR}\left(500 \mathrm{MHz}, \mathrm{CDCl}_{3}\right) \delta$ 7.74-7.71 (m, 3H), $7.48(\mathrm{~d}, J=8.7 \mathrm{~Hz}, 1 \mathrm{H}), 7.18(\mathrm{dd}, J$ $=8.9,2.2 \mathrm{~Hz}, 1 \mathrm{H}), 7.14(\mathrm{~s}, 1 \mathrm{H}), 4.96(\mathrm{q}, J=7.1 \mathrm{~Hz}, 1 \mathrm{H}), 3.93(\mathrm{~s}, 3 \mathrm{H}), 2.02(\mathrm{~d}, J=7.0$ $\mathrm{Hz}, 3 \mathrm{H}) .{ }^{19} \mathrm{~F} \mathrm{NMR}\left(471 \mathrm{MHz}, \mathrm{CDCl}_{3}\right) \delta-33.4$ (s, 3F). ${ }^{13} \mathrm{C} \mathrm{NMR}\left(126 \mathrm{MHz}, \mathrm{CDCl}_{3}\right) \delta$ 158.1, 136.2, 134.1, 129.4, 128.7, 127.6, 125.9, 125.7, 123.4 (q, $J=331.4 \mathrm{~Hz}), 119.3$, 105.8, 55.4, 42.2, 23.2. IR (KBr): 3058, 3004, 2963, 2929, 2874, 2847, 1628, 1605, 1504, 1486, 1457, 1449, 1439, 1418, 1392, 1347, 1267, 1235, 1196, 1163, 1146, 1105, 1066, 1029, 958, 923, 892, 856, 814, 752, 738, $680 \mathrm{~cm}^{-1}$. HRMS-ESI (m/z) calcd. for $\mathrm{C}_{14} \mathrm{H}_{14} \mathrm{~F}_{3} \mathrm{OSe}\left([\mathrm{M}+\mathrm{H}]^{+}\right)$: 335.0156; found: 335.0165.<smiles>CC([Se]C(F)(F)F)c1cccc(C(=O)c2ccccc2)c1</smiles>

Phenyl(3-(1-((trifluoromethyl)selanyl)ethyl)phenyl)methanone (3ai). Light yellow liquid (22.5 mg, $31 \%$ yield; $32.9 \mathrm{mg}, 46 \%$ yield), a mixture of petroleum ether/ethyl acetate $=40: 1(\mathrm{v} / \mathrm{v})$ as eluents for column chromatography. ${ }^{1} \mathrm{H}$ NMR $(500 \mathrm{MHz}$, $\left.\mathrm{CDCl}_{3}\right) \delta 7.82-7.79(\mathrm{~m}, 3 \mathrm{H}), 7.70(\mathrm{~d}, J=7.6 \mathrm{~Hz}, 1 \mathrm{H}), 7.63-7.60(\mathrm{~m}, 2 \mathrm{H}), 7.50(\mathrm{t}, J=$ $7.6 \mathrm{~Hz}, 2 \mathrm{H}), 7.47$ (t, $J=7.7 \mathrm{~Hz}, \mathrm{H}), 4.83(\mathrm{q}, J=7.1 \mathrm{~Hz}, 1 \mathrm{H}), 1.92(\mathrm{~d}, J=7.1 \mathrm{~Hz}, 3 \mathrm{H})$. ${ }^{19} \mathrm{~F}$ NMR $\left(471 \mathrm{MHz}, \mathrm{CDCl}_{3}\right) \delta-33.4$ (s, 3F). ${ }^{13} \mathrm{C} \mathrm{NMR}\left(126 \mathrm{MHz}, \mathrm{CDCl}_{3}\right) \delta 196.2$, $142.1,138.2,137.3,132.7,131.2,130.1,129.7,128.8,128.6,128.4,123.1$ (q, $J=$ 332.1 Hz), 41.2, 22.9. IR (KBr): 3062, 3029, 2966, 2925, 2871, 2852, 1662, 1598 , 1580, 1482, 1470, 1448, 1436, 1381, 1318, 1285, 1271, 1208, 1179, 1117, 1097, 1076, 1044, 981, 950, 819, 802, 785, 738, 722, 705, 691, $648 \mathrm{~cm}^{-1}$. HRMS-ESI (m/z) calcd. for $\mathrm{C}_{16} \mathrm{H}_{13} \mathrm{~F}_{3} \mathrm{NaOSe}\left([\mathrm{M}+\mathrm{Na}]^{+}\right): 380.9976$; found: 380.9972 .<smiles>CC(c1ccc2c(c1)Cc1cccnc1O2)C(F)(F)F</smiles>

7-(1-((Trifluoromethyl)selanyl)ethyl)-5H-chromeno[2,3-b]pyridine (3aj). White solid 
(21.1 mg, 30\% yield; $37.2 \mathrm{mg}, 52 \%$ yield), a mixture of hexane/dichloromethane = 1:2 (v/v) as eluents for column chromatography. M.p.: 79-81 ${ }^{\circ} \mathrm{C} .{ }^{1} \mathrm{H}$ NMR $(500 \mathrm{MHz}$, $\left.\mathrm{CDCl}_{3}\right) \delta 8.18(\mathrm{~d}, J=4.7 \mathrm{~Hz}, 1 \mathrm{H}), 7.54(\mathrm{~d}, J=7.3 \mathrm{~Hz}, 1 \mathrm{H}), 7.23(\mathrm{dd}, J=8.4,1.9 \mathrm{~Hz}$, $1 \mathrm{H}), 7.18(\mathrm{~s}, 1 \mathrm{H}), 7.12(\mathrm{~d}, J=8.4 \mathrm{~Hz}, 1 \mathrm{H}), 7.04(\mathrm{dd}, J=7.3,4.9 \mathrm{~Hz}, 1 \mathrm{H}), 4.77(\mathrm{q}, J=$ $7.0 \mathrm{~Hz}, 1 \mathrm{H}), 4.10(\mathrm{~s}, 2 \mathrm{H}), 1.91(\mathrm{~d}, J=7.0 \mathrm{~Hz}, 3 \mathrm{H}) .{ }^{19} \mathrm{~F}$ NMR $\left(471 \mathrm{MHz}, \mathrm{CDCl}_{3}\right) \delta$ -33.5 (s, 3F). ${ }^{13} \mathrm{C}$ NMR (126 MHz, $\left.\mathrm{CDCl}_{3}\right) \delta 158.3,151.2,146.8,138.4,136.6,127.4$, 127.0, 123.2 (q, $J=332.5 \mathrm{~Hz}), 120.0,119.9,117.6,115.1,41.2,28.1,23.2$. IR (KBr): 2992, 2970, 2922, 2870, 2850, 1671, 1646, 1605, 1578, 1502, 1448, 1427, 1380, 1318 , 1304, 1282, 1223, 1190, 1128, 1097, 1062, 1042, 978, 929, 890, 884, 822, 787, 748, 737, 721, 675, $618 \mathrm{~cm}^{-1}$. HRMS-ESI $(\mathrm{m} / \mathrm{z})$ calcd. for $\mathrm{C}_{15} \mathrm{H}_{12} \mathrm{~F}_{3} \mathrm{NNaOSe}\left([\mathrm{M}+\mathrm{Na}]^{+}\right)$: 381.9928; found: 381.9924 .

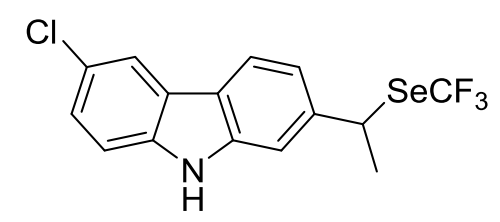

6-Chloro-2-(1-((trifluoromethyl)selanyl)ethyl)-9H-carbazole (3ak). Light yellow solid (37.7 mg, 50\% yield), a mixture of petroleum ether/ethyl acetate $=15: 1(\mathrm{v} / \mathrm{v})$ as eluents for column chromatography. M.p.: $106-108{ }^{\circ} \mathrm{C} .{ }^{1} \mathrm{H} \mathrm{NMR}\left(500 \mathrm{MHz}, \mathrm{CDCl}_{3}\right) \delta$ 8.05 (brs, 1H), 8.00 (s, 1H), 7.97 (d, $J=8.1 \mathrm{~Hz}, 1 \mathrm{H}), 7.42$ (s, 1H), 7.34 (dd, $J=8.6$, $1.7 \mathrm{~Hz}, 1 \mathrm{H}), 7.34(\mathrm{~d}, J=8.5 \mathrm{~Hz}, 1 \mathrm{H}), 7.27(\mathrm{~m}, 1 \mathrm{H}), 4.97$ (q, J = 7.0 Hz, 1H), 2.02 (d, $J=7.0 \mathrm{~Hz}, 3 \mathrm{H}) .{ }^{19} \mathrm{~F}$ NMR $\left(471 \mathrm{MHz}, \mathrm{CDCl}_{3}\right) \delta-33.5(\mathrm{~s}, 3 \mathrm{~F}) .{ }^{13} \mathrm{C} \mathrm{NMR}(126 \mathrm{MHz}$, $\left.\mathrm{CDCl}_{3}\right) \delta 140.1,139.9,138.2,126.2,125.3,124.2,123.3(\mathrm{q}, J=332.4 \mathrm{~Hz}), 122.3$, 120.9, 120.1, 119.3, 111.7, 109.4, 42.5, 23.6. IR (KBr): 3414, 2925, 2854, 1627, 1612, 1473, 1450, 1429, 1339, 1328, 1274, 1244, 1148, 1099, 1069, 1040, 930, 876, 864, 827, 806, 738, 731, 698, 664, $585 \mathrm{~cm}^{-1}$. HRMS-ESI (m/z) calcd. for $\mathrm{C}_{15} \mathrm{H}_{12} \mathrm{ClF}_{3} \mathrm{NSe}$ $\left([\mathrm{M}+\mathrm{H}]^{+}\right): 376.9775$; found: 376.9780 .

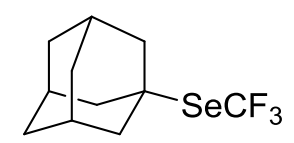

$\left((3 \mathrm{~s}, 5 \mathrm{~s}, 7 \mathrm{~s})\right.$-Adamantan-1-yl)(trifluoromethyl)selane (3al) ${ }^{4 \mathrm{c}}$ Colorless liquid (20.3 mg, $36 \%$ yield $\left(55 \%{ }^{19} \mathrm{~F}\right.$ NMR yield)), petroleum ether as eluent for column chromatography. ${ }^{1} \mathrm{H}$ NMR (500 MHz, $\left.\mathrm{CDCl}_{3}\right) \delta 2.23$ (d, $\left.J=2.5 \mathrm{~Hz}, 6 \mathrm{H}\right), 2.10(\mathrm{~s}, 3 \mathrm{H})$, $1.77(\mathrm{~s}, 6 \mathrm{H}) .{ }^{19} \mathrm{~F}$ NMR $\left(471 \mathrm{MHz}, \mathrm{CDCl}_{3}\right) \delta-28.3$ (s, 3F). ${ }^{13} \mathrm{C}$ NMR (126 MHz, 
$\left.\mathrm{CDCl}_{3}\right) \delta 123.8(\mathrm{q}, J=332.1 \mathrm{~Hz}), 53.1,44.8,36.0,30.7$.

Procedure for a large scale synthesis of 3a: In a nitrogen-filled glovebox, a round-bottom flask was charged with 3-([1,1'-biphenyl]-4-yl)propanoic acid (1a, $441.0 \mathrm{mg}, 1.95 \mathrm{mmol}), \mathrm{NFSI}(615.0 \mathrm{mg}, 1.95 \mathrm{mmol}),\left[\mathrm{Me}_{4} \mathrm{~N}\right]\left[\mathrm{SeCF}_{3}\right](333.0 \mathrm{mg}, 1.50$ mmol), [Mes-Acr-Ph] $\left.\mathrm{BF}_{4}\right](7.5 \mathrm{mg}, 0.015 \mathrm{mmol})$, and $\mathrm{CH}_{3} \mathrm{CN}(12 \mathrm{~mL})$ with vigorous stirring. The reaction mixture was irradiated by $2 \times 10 \mathrm{~W}$ blue LEDs $(7 \mathrm{~cm}$ away, with cooling fan to keep the reaction at room temperature) for 24 hours, quenched with water, and concentrated under reduced pressure. The residue was purified by flash column chromatography on silica gel using petroleum ether as eluent to give the trifluoromethylselenolated product $\mathbf{3 a}(371.0 \mathrm{mg}, 75 \%$ yield) as a white solid.

\section{The control experiments for mechanistic insights}

4.1. The standard reactions of 1a, $2 a$ and NFSI in the presence of different radical inhibitors.

\section{Table S8}

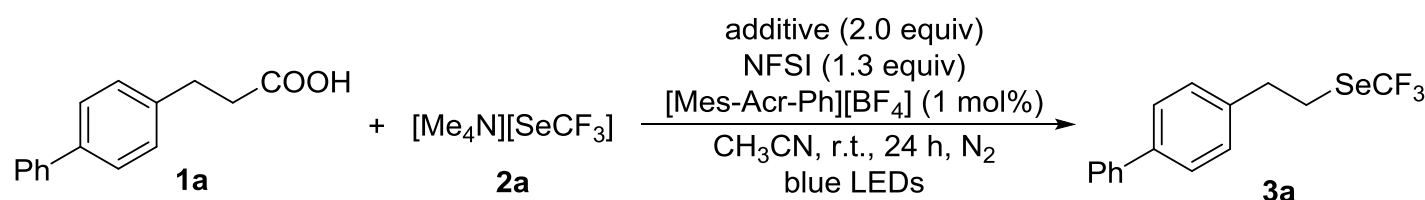

(1.3 equiv)

$2 \mathrm{a}$ blue LEDs

$3 a$

\begin{tabular}{ccc}
\hline Entry $^{a}$ & Additive & Yield (3a, \%) \\
\hline 1 & none & 86 \\
2 & BHT & 21 \\
3 & diallyl-PTSA & 22 \\
4 & 1,1-diphenylethylene & 51 \\
5 & TEMPO & 0 \\
6 & 1,3-dinitrobenzene & 85 \\
7 & 1,4-dinitrobenzene & 87
\end{tabular}

${ }^{a}$ Reaction conditions: 1a $(0.26 \mathrm{mmol}), 2 \mathrm{2a}(0.20 \mathrm{mmol})$, NFSI $(0.26 \mathrm{mmol})$, [Mes-Acr-Ph] $\left[\mathrm{BF}_{4}\right](0.002 \mathrm{mmol})$, additive $(0.40 \mathrm{mmol}), \mathrm{CH}_{3} \mathrm{CN}(2 \mathrm{~mL})$, r.t., $\mathrm{N}_{2}, 24 \mathrm{~h}$, blue LEDs $(10 \mathrm{~W} \times 2,435-455 \mathrm{~nm})$. The yields were determined by HPLC using $3 \mathbf{a}$ as an external standard $\left(\mathrm{t}_{\mathrm{R}}=8.34 \mathrm{~min}, \lambda_{\max }=253 \mathrm{~nm}\right.$, water/methanol $\left.(\mathrm{v} / \mathrm{v})=10: 90\right)$. 
4.2. ${ }^{19}$ F NMR analyses of the reaction mixtures under standard conditions at different reaction times with or without radical trap.

Procedure: In a nitrogen-filled glovebox, sealed tubes were charged with 1a $(0.26$ $\mathrm{mmol}), \mathrm{NFSI}(0.26 \mathrm{mmol}),\left[\mathrm{Me}_{4} \mathrm{~N}\right]\left[\mathrm{SeCF}_{3}\right](0.20 \mathrm{mmol}),[\mathrm{Mes}-\mathrm{Acr}-\mathrm{Ph}]\left[\mathrm{BF}_{4}\right](0.002$ $\mathrm{mmol})$ and $\mathrm{CH}_{3} \mathrm{CN}(2 \mathrm{~mL})$ with vigorous stirring. The mixtures were irradiated by $2 \times$ $10 \mathrm{~W}$ blue LEDs (7 cm away) at room temperature for different times (1, 4, 8, 12, and $24 \mathrm{~h}$ ) and analyzed by ${ }^{19} \mathrm{~F}$ NMR using $\mathrm{PhOCF}_{3}$ as an internal standard.

Figure 1. The ${ }^{19} \mathrm{~F}$ NMR spectrum of the standard reaction mixture for $1 \mathrm{~h}$ (41.7 $\mathrm{mg}$ of $\mathrm{PhOCF}_{3}(0.257 \mathrm{mmol})$ was used as an internal standard $)$.

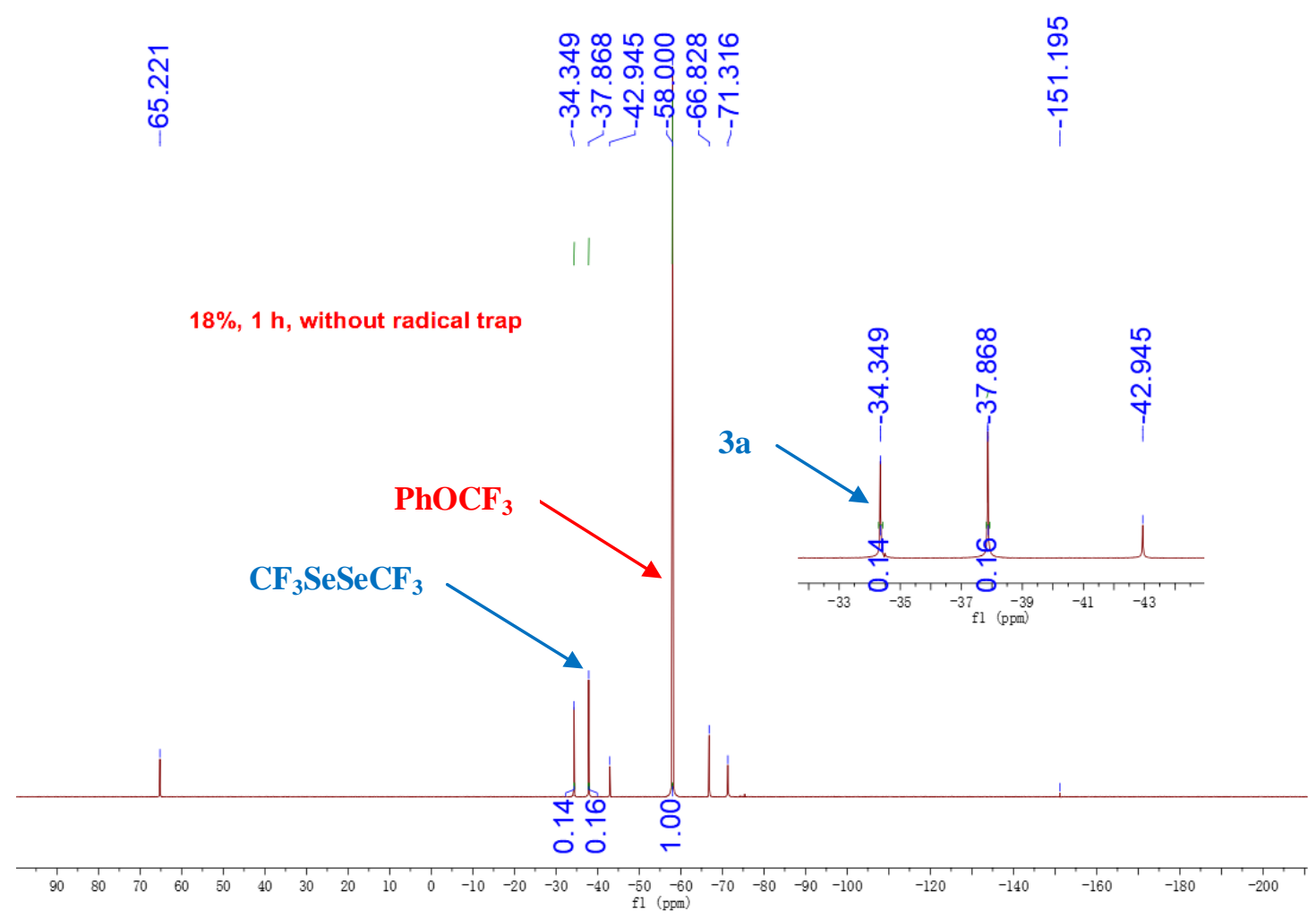

Figure 2. The ${ }^{19} \mathrm{~F}$ NMR spectrum of the standard reaction mixture for $4 \mathrm{~h}$ (25.3 $\mathrm{mg}$ of $\mathrm{PhOCF}_{3}(0.156 \mathrm{mmol})$ was used as an internal standard $)$. 


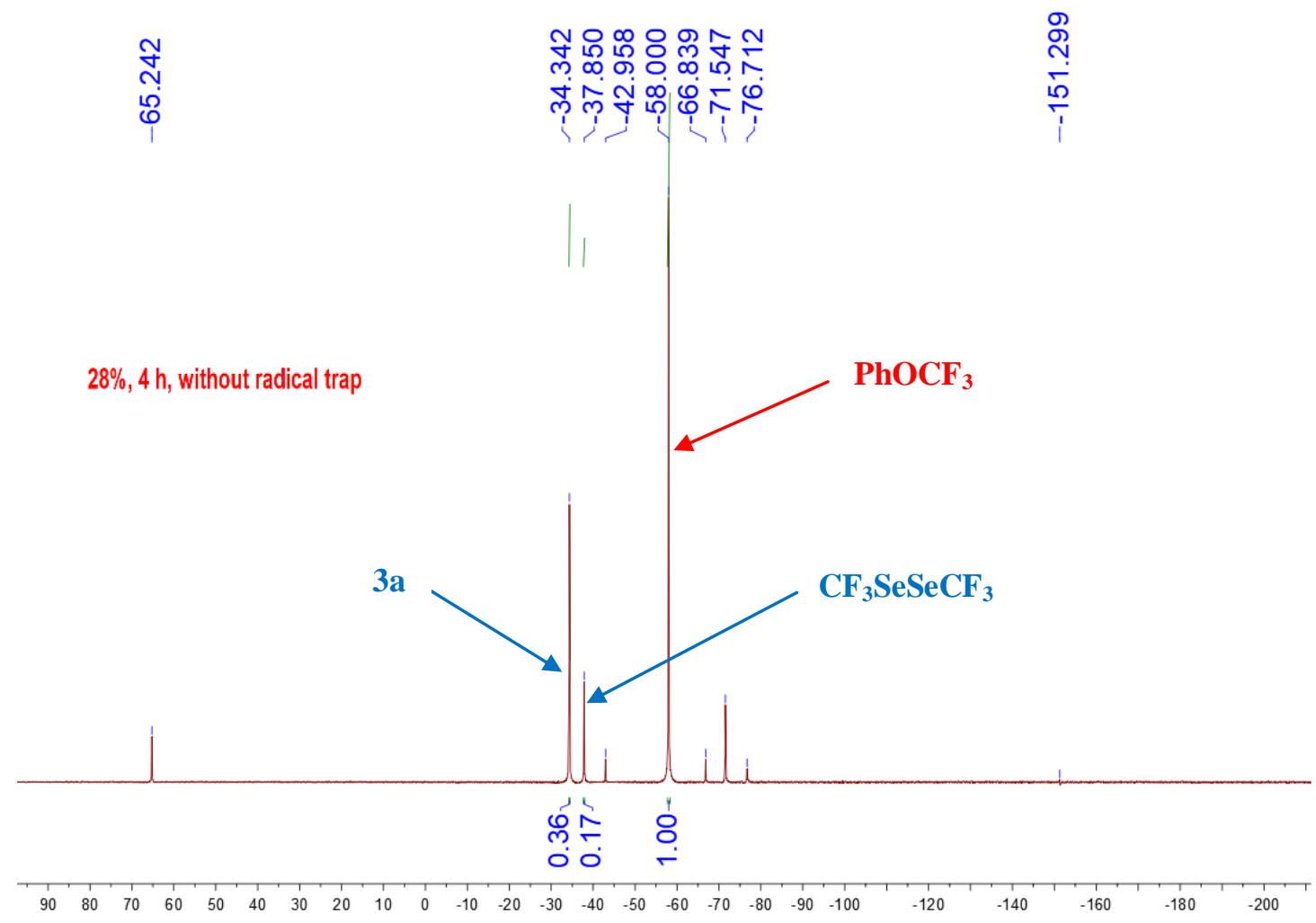

Figure 3. The ${ }^{19} \mathrm{~F}$ NMR spectrum of the standard reaction mixture for $8 \mathrm{~h}(26.6 \mathrm{mg}$ of $\mathrm{PhOCF}_{3}(0.164 \mathrm{mmol})$ was used as an internal standard $)$.
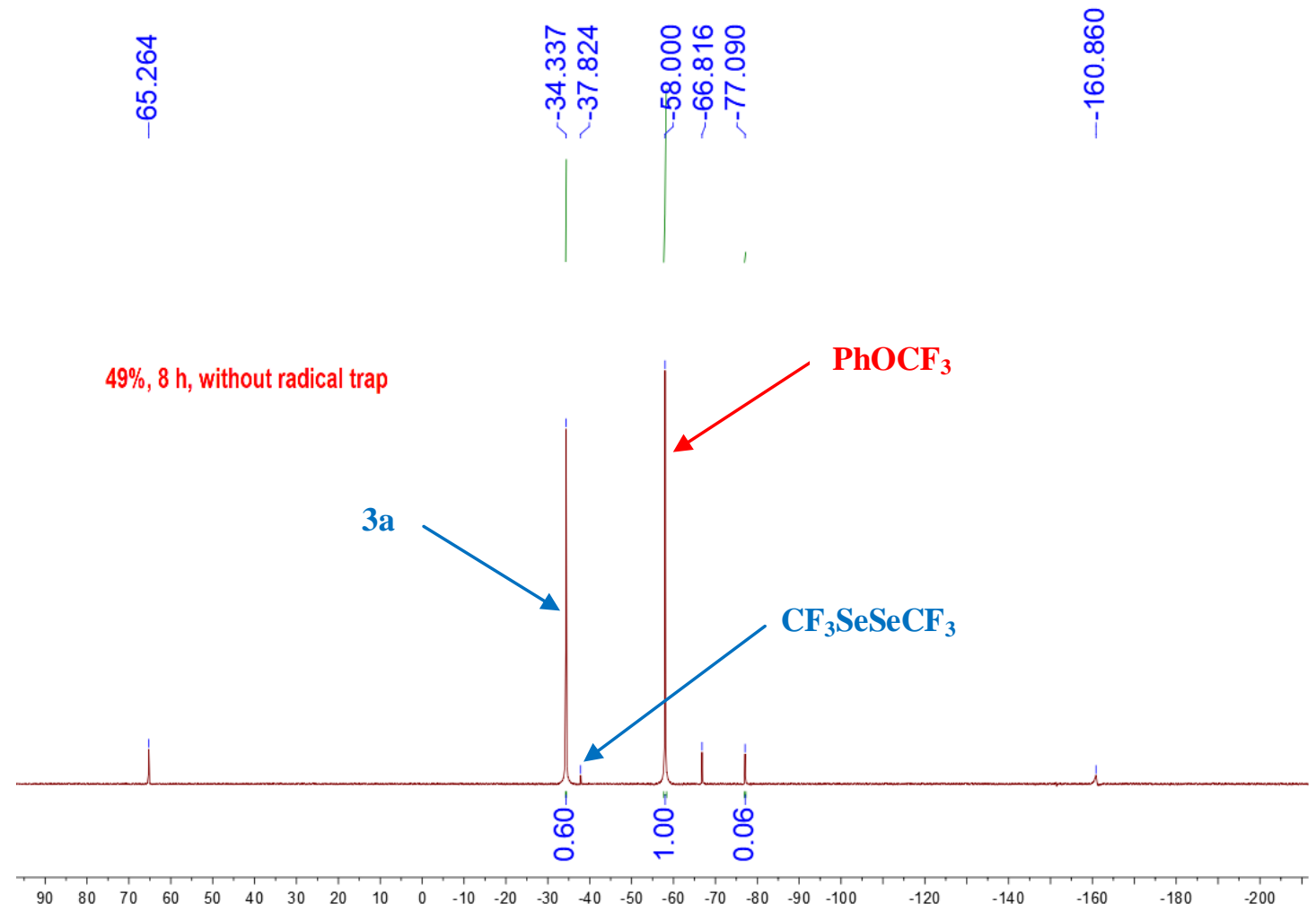

Figure 4. The ${ }^{19} \mathrm{~F}$ NMR spectrum of the standard reaction mixture for $12 \mathrm{~h}(35.6 \mathrm{mg}$ 
of $\mathrm{PhOCF}_{3}(0.220 \mathrm{mmol})$ was used as an internal standard $)$.

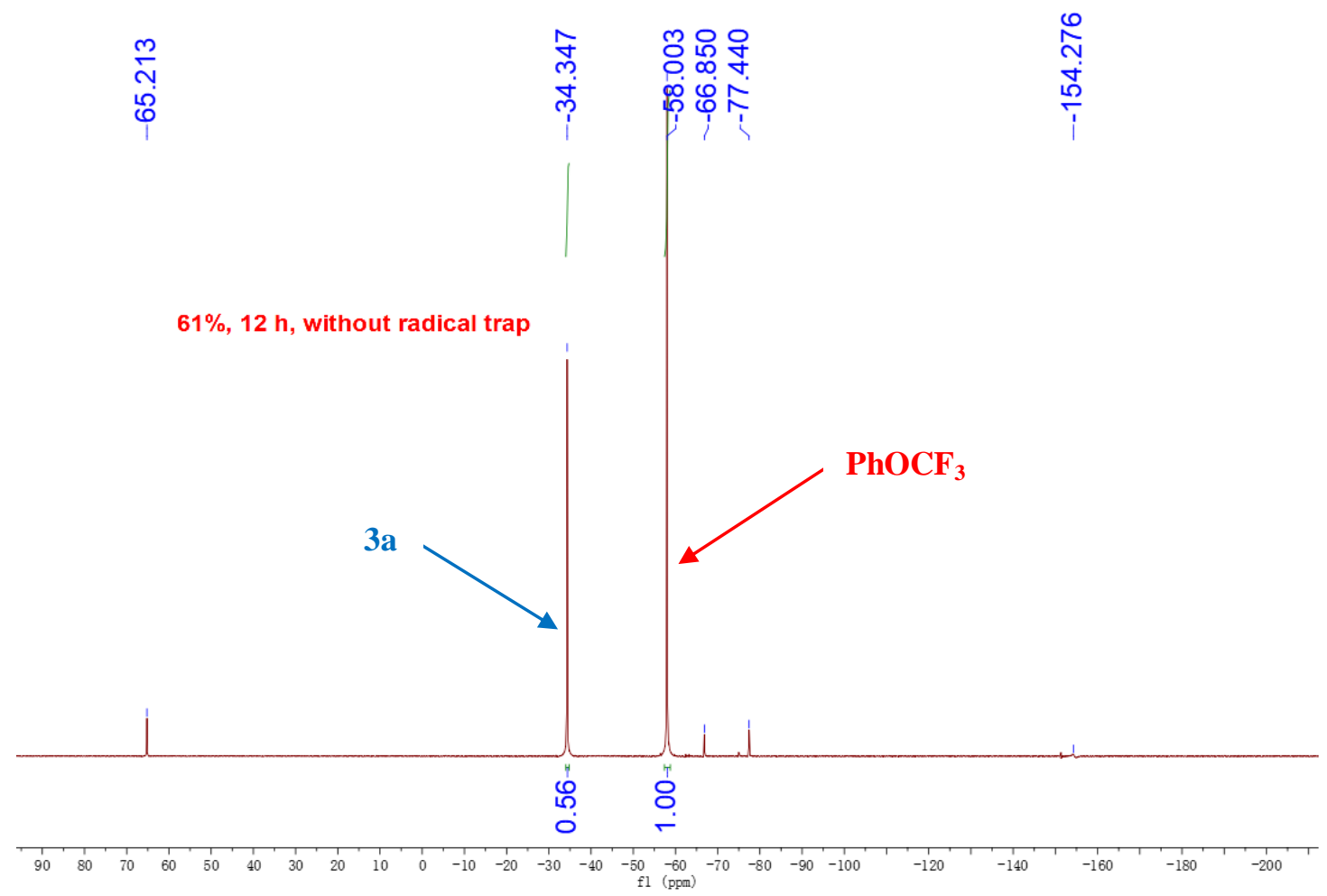

Figure 5. The ${ }^{19} \mathrm{~F}$ NMR spectrum of the standard reaction mixture for $24 \mathrm{~h}(48.0 \mathrm{mg}$ of $\mathrm{PhOCF}_{3}(0.296 \mathrm{mmol})$ was used as an internal standard).

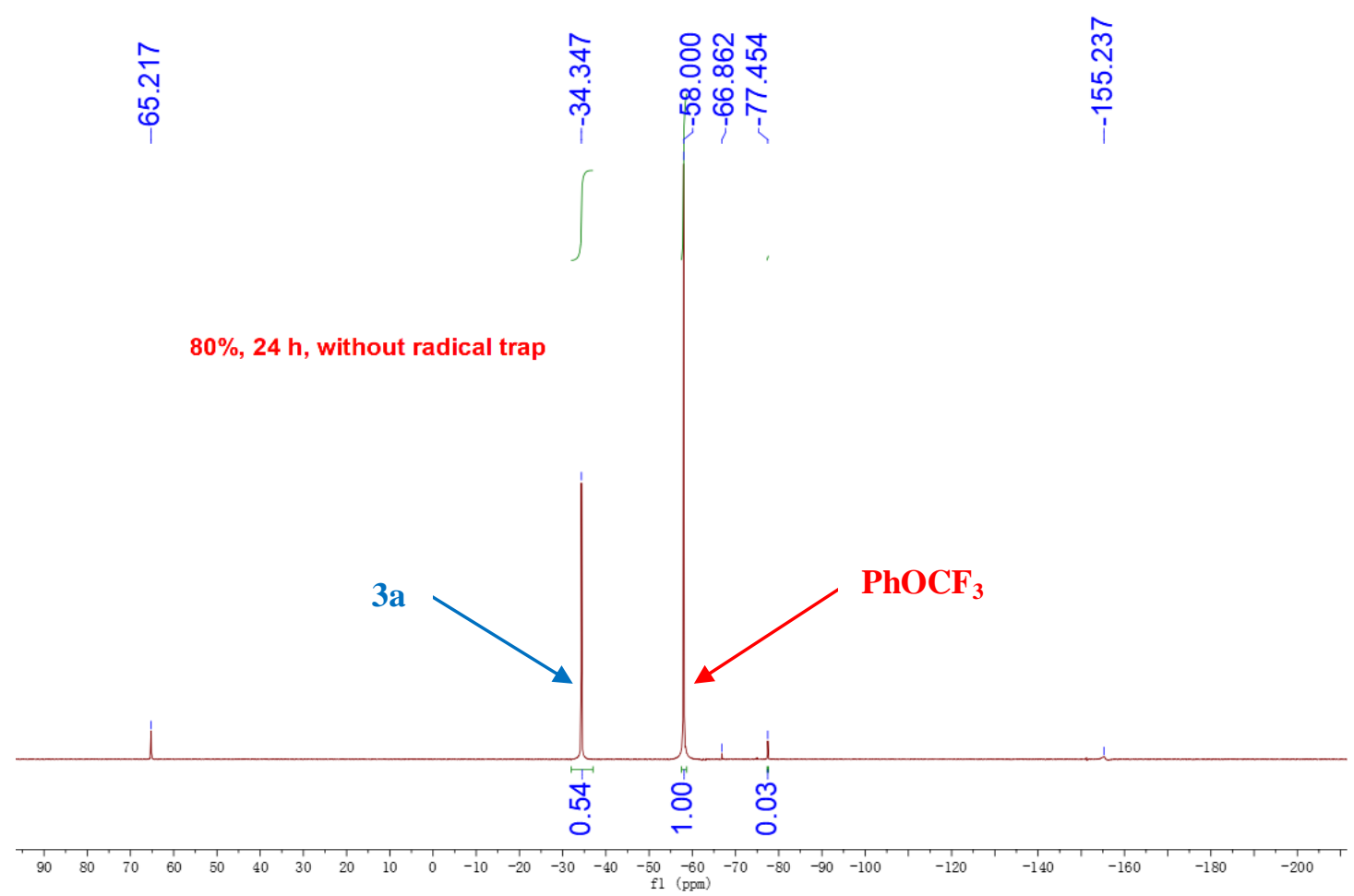

Figure 6. The ${ }^{19} \mathrm{~F}$ NMR spectrum of the reaction mixture for $24 \mathrm{~h}$ in the presence of 
TEMPO (37.1 mg of $\mathrm{PhOCF}_{3}(0.229 \mathrm{mmol})$ was used as an internal standard).

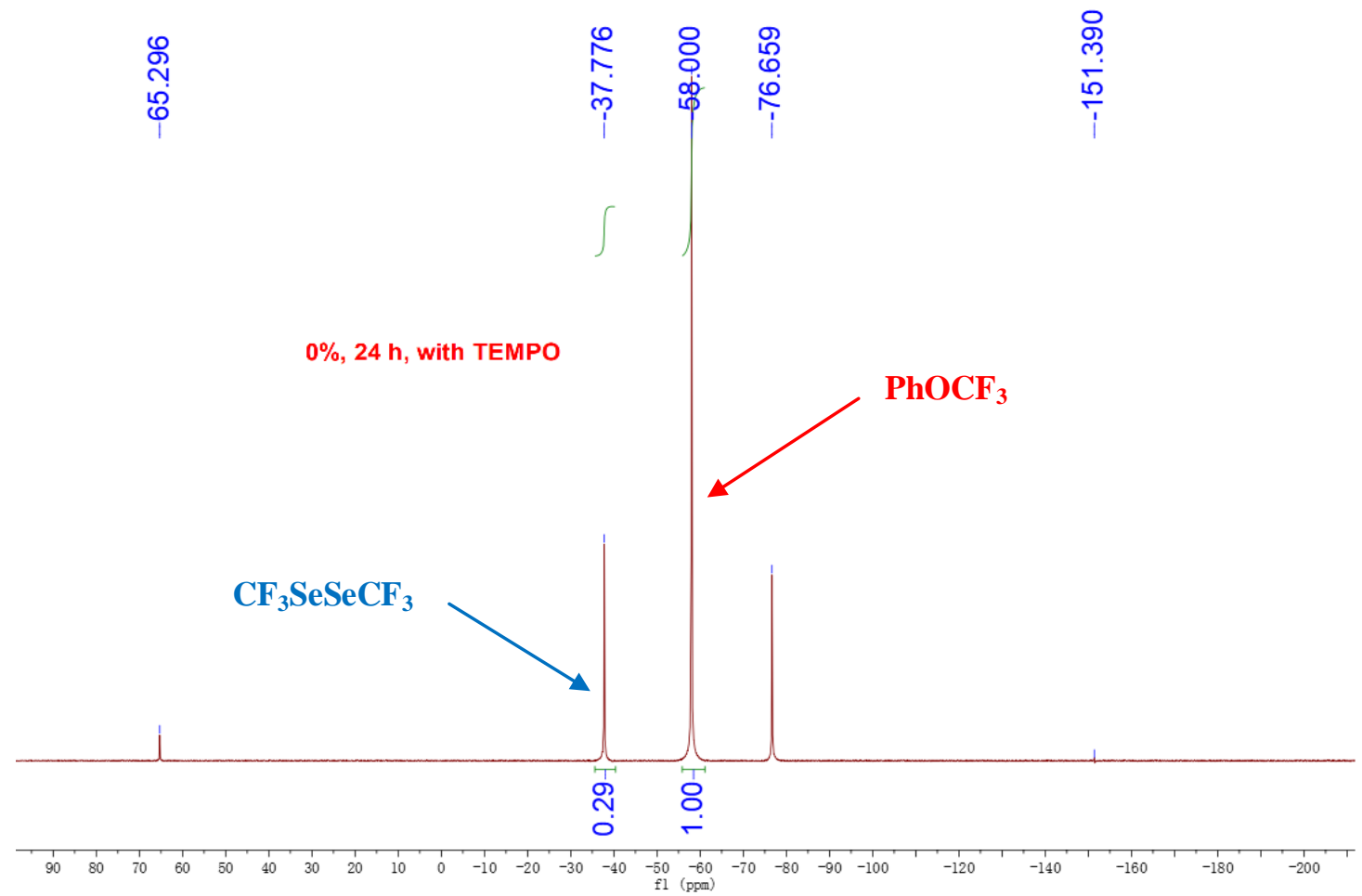

Figure 7. The combination of ${ }^{19} \mathrm{~F}$ NMR spectra in Figure 1-6
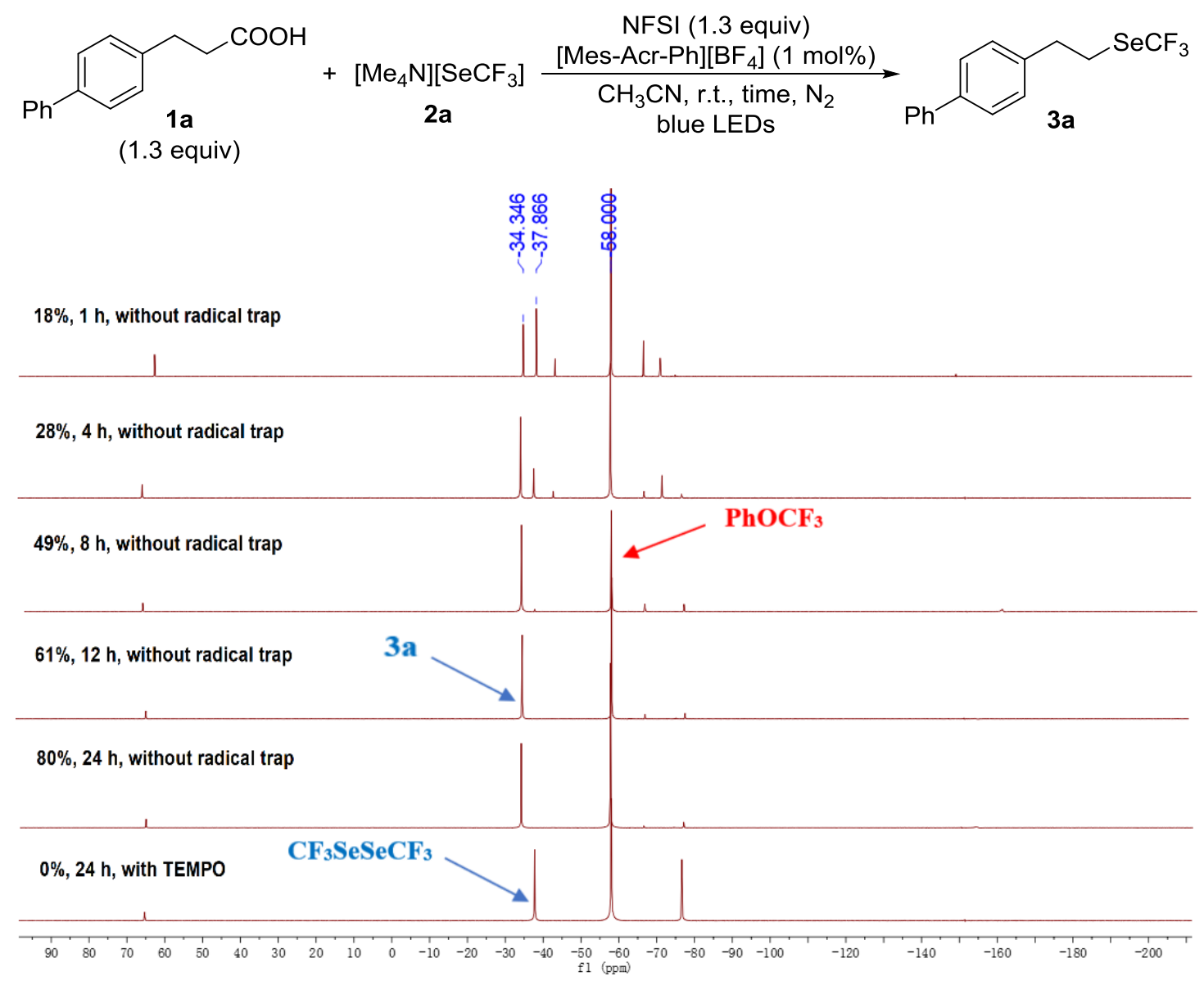


\subsection{The light on-off experiments}

The light on-off experiments were carried out under the standard reaction conditions using a mixture of $1 \mathbf{a}(0.26 \mathrm{mmol}), \mathrm{NFSI}(0.26 \mathrm{mmol}),\left[\mathrm{Me}_{4} \mathrm{~N}\right]\left[\mathrm{SeCF}_{3}\right](0.20 \mathrm{mmol})$, [Mes-Acr-Ph] $\left[\mathrm{BF}_{4}\right](0.002 \mathrm{mmol})$ and $\mathrm{CH}_{3} \mathrm{CN}(2 \mathrm{~mL})$. Light was switched on and off on $1.5 \mathrm{~h}$ intervals, and the reaction mixture was monitored by HPLC after each period. As shown in Table S8 and the chart, during light-off periods of the reaction, the formation of 3a was almost completely halted. However, it could be restarted under irradiation.

\section{Table S9}

\begin{tabular}{c|ccccccccc}
\hline Time/h & 0 & 1.5 & 3.0 & 4.5 & 6.0 & 7.5 & 9.0 & 10.5 & 12 \\
\hline $\begin{array}{c}\text { Yield } \\
(\mathbf{3 a}) \%\end{array}$ & 0 & 15 & 15 & 25 & 25 & 33 & 34 & 45 & 46 \\
\hline
\end{tabular}

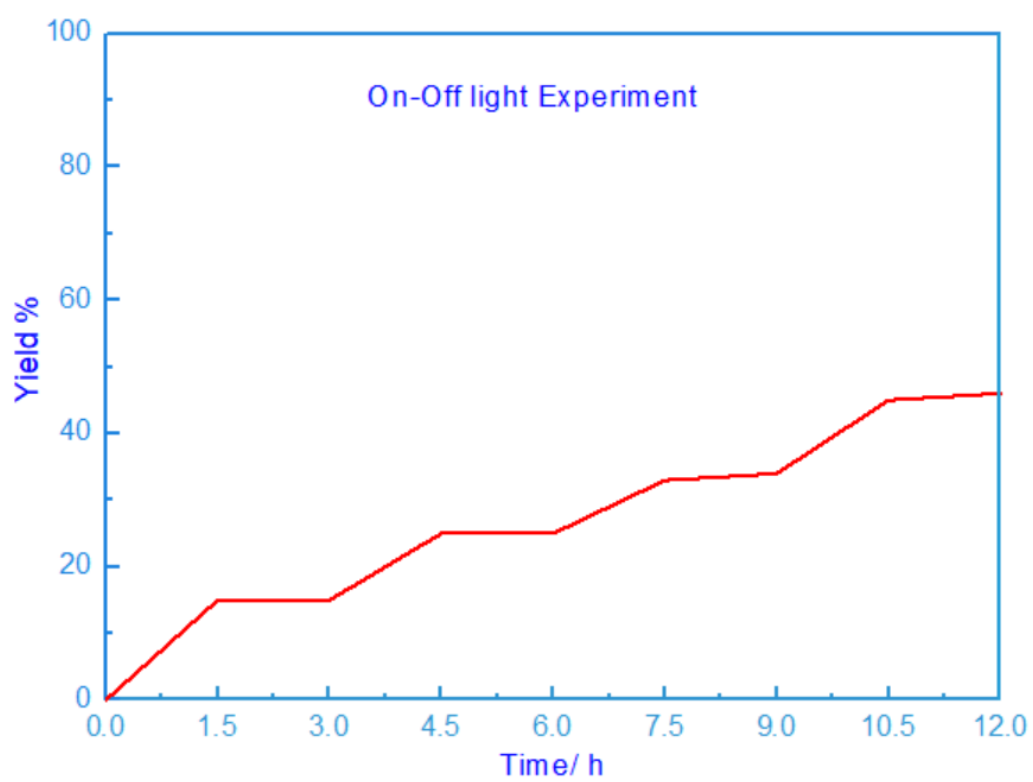

Figure 8. The light on-off experiments with the reaction of $1 \mathrm{a}$ and $2 \mathrm{a}$.

4.4. GC-MS analysis of the standard reaction mixture of 1a, NFSI, $\left[\mathrm{Me}_{4} \mathrm{~N}\right]\left[\mathrm{SeCF}_{3}\right]$, and [Mes-Acr-Ph] $\left[\mathrm{BF}_{4}\right]$ at room temperature for $1 \mathrm{~h}$.

Procedure: In a nitrogen-filled glovebox, a sealed tube was charged with 1a $(0.26$ mmol), NFSI (0.26 mmol), [ $\left.\mathrm{Me}_{4} \mathrm{~N}\right]\left[\mathrm{SeCF}_{3}\right](0.20 \mathrm{mmol}),[\mathrm{Mes}-\mathrm{Acr}-\mathrm{Ph}]\left[\mathrm{BF}_{4}\right](0.002$ mmol) and $\mathrm{CH}_{3} \mathrm{CN}(2 \mathrm{~mL})$ with vigorous stirring. The mixture was irradiated by $2 \times$ 
$10 \mathrm{~W}$ blue LEDs ( $7 \mathrm{~cm}$ away) at room temperature for $1 \mathrm{~h}$ and analyzed by a GC-MS instrument (Agilent 222-5532LTM DB-5ms).

Figure 9. Copies of the GC-MS spectra of the above reaction mixture.

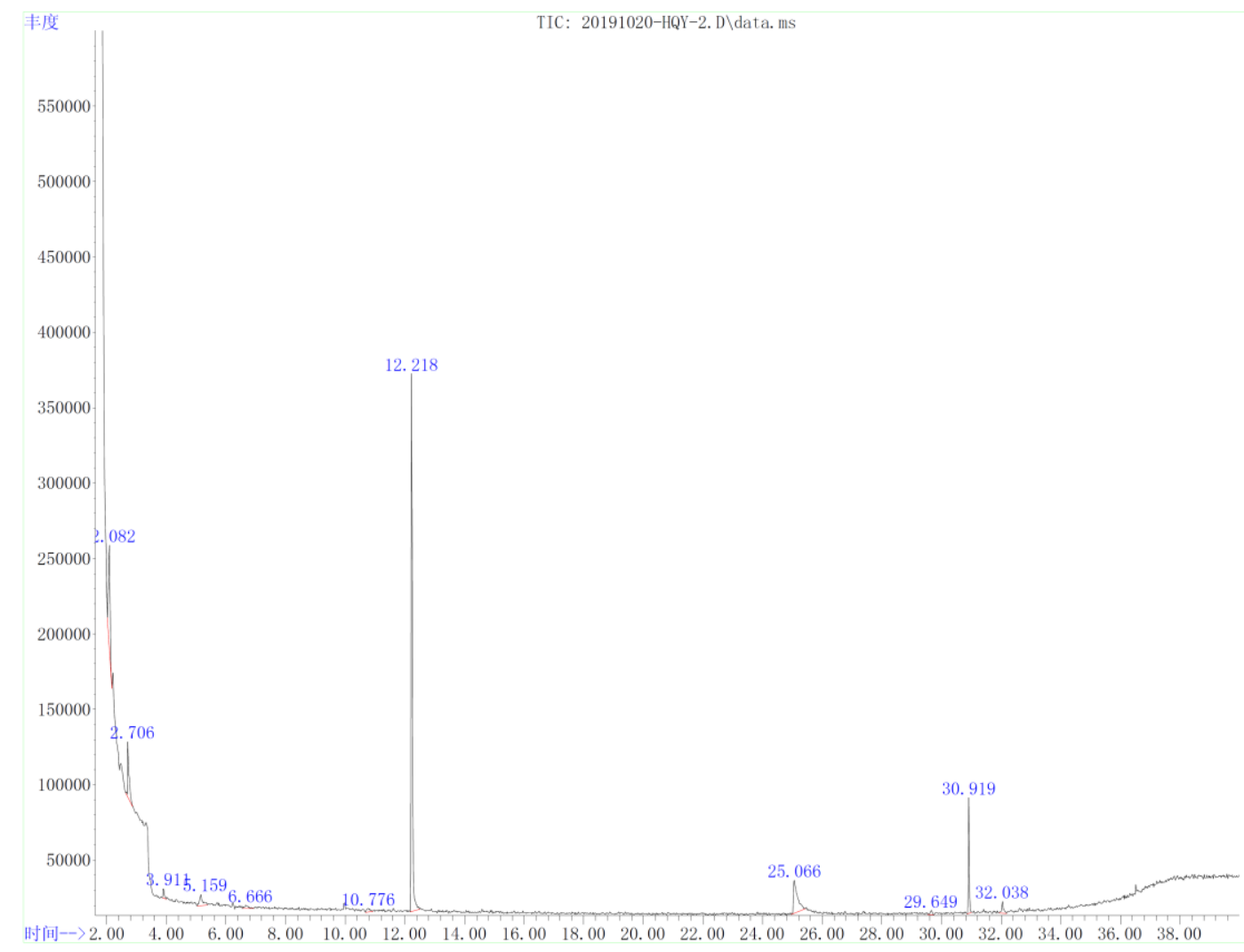

The MS spectrum at $t_{R}$ (retention time $)=2.08$ minutes.

$\mathrm{CF}_{3} \mathrm{SeSeCF}_{3}(\mathrm{~m} / \mathrm{z}=297.8)$ was detected (see below):

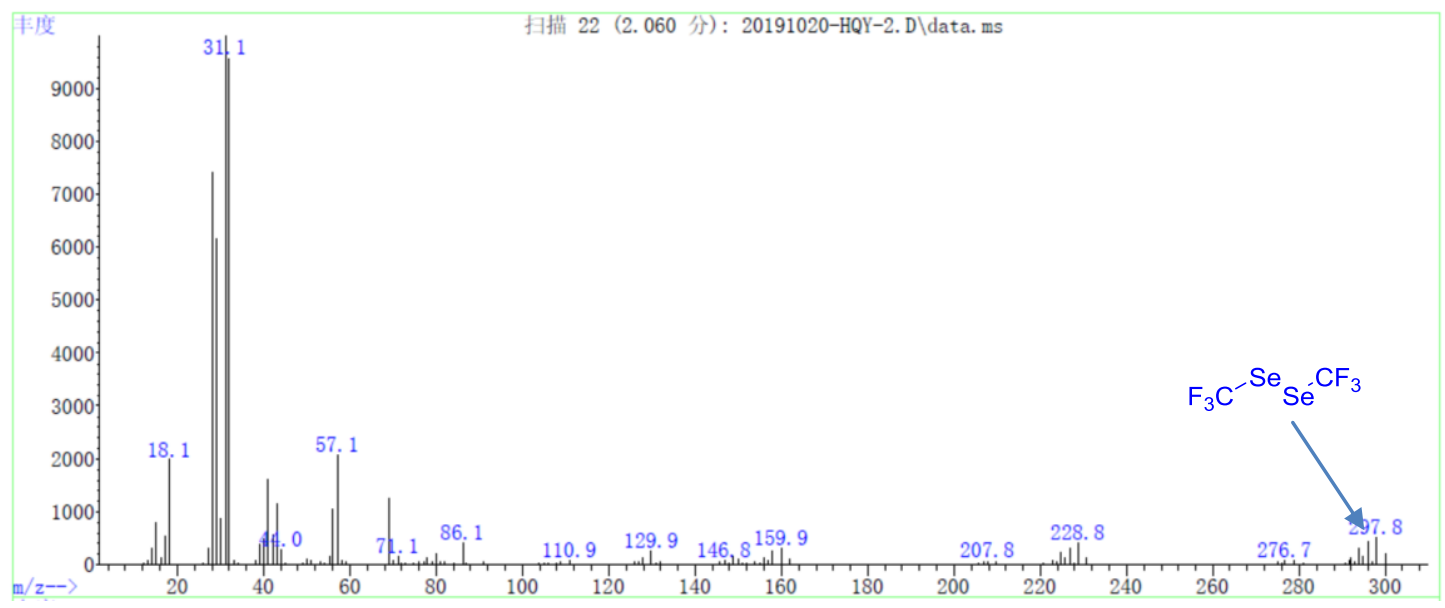

The MS spectrum at $t_{R}$ (retention time $)=12.22$ minutes. 
$\mathrm{PhSO}_{2} \mathrm{~F}(\mathrm{~m} / \mathrm{z}=160.0)$ was detected (see below):

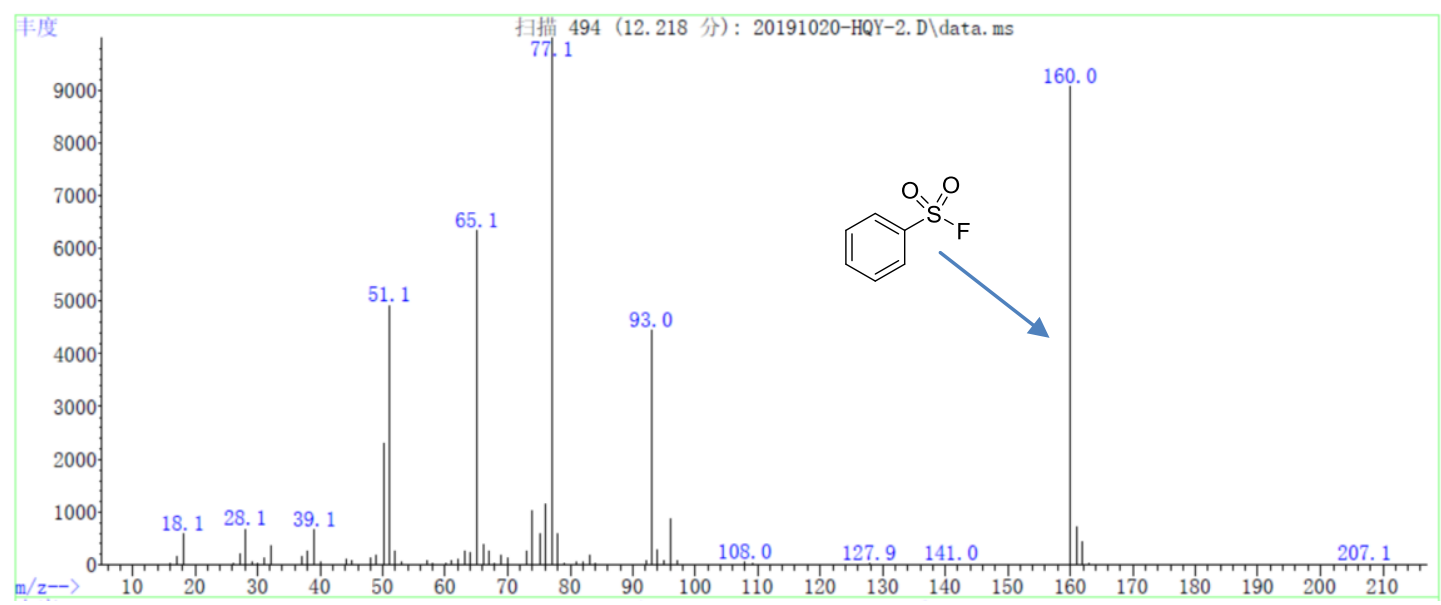

The MS spectrum at $\mathbf{t}_{\mathbf{R}}$ (retention time $)=25.07$ minutes .

$\mathrm{PhSO}_{2} \mathrm{NH}_{2}(\mathrm{~m} / \mathrm{z}=157.0)$ was detected (see below):

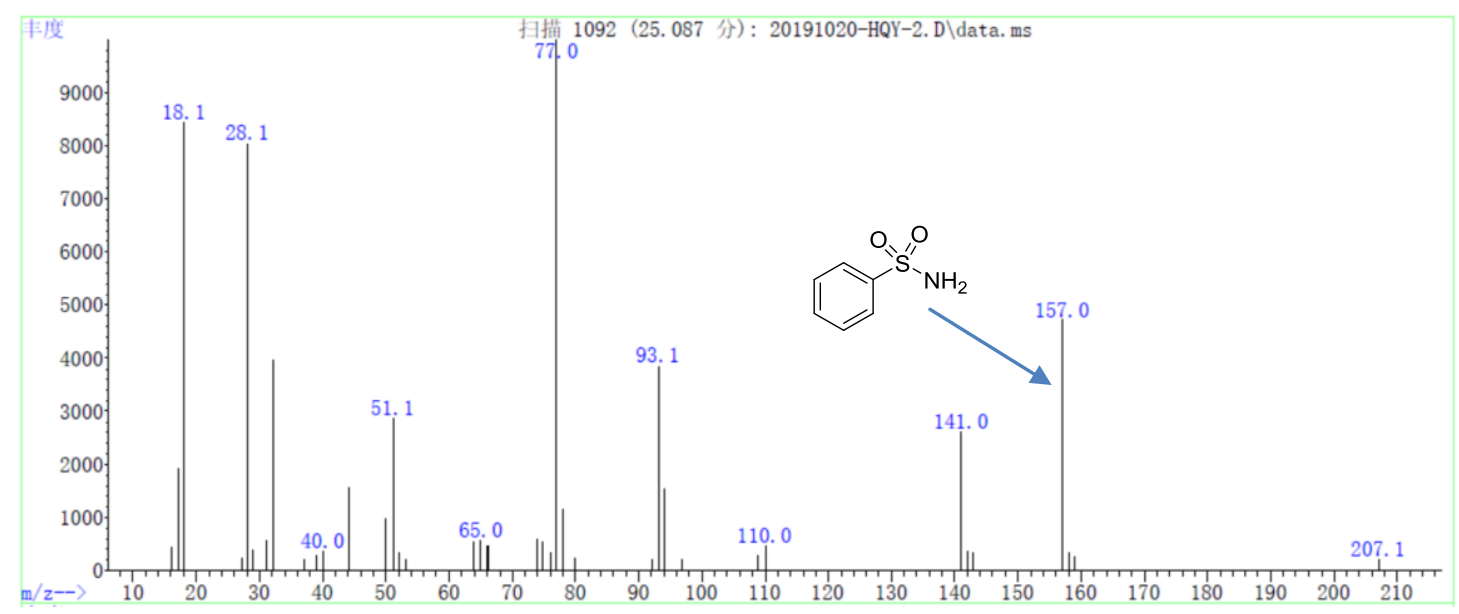

The MS spectrum at $\mathbf{t}_{\mathbf{R}}$ (retention time $)=30.92$ minutes .

3a $(\mathrm{m} / \mathrm{z}=330.0)$ was detected (see below):

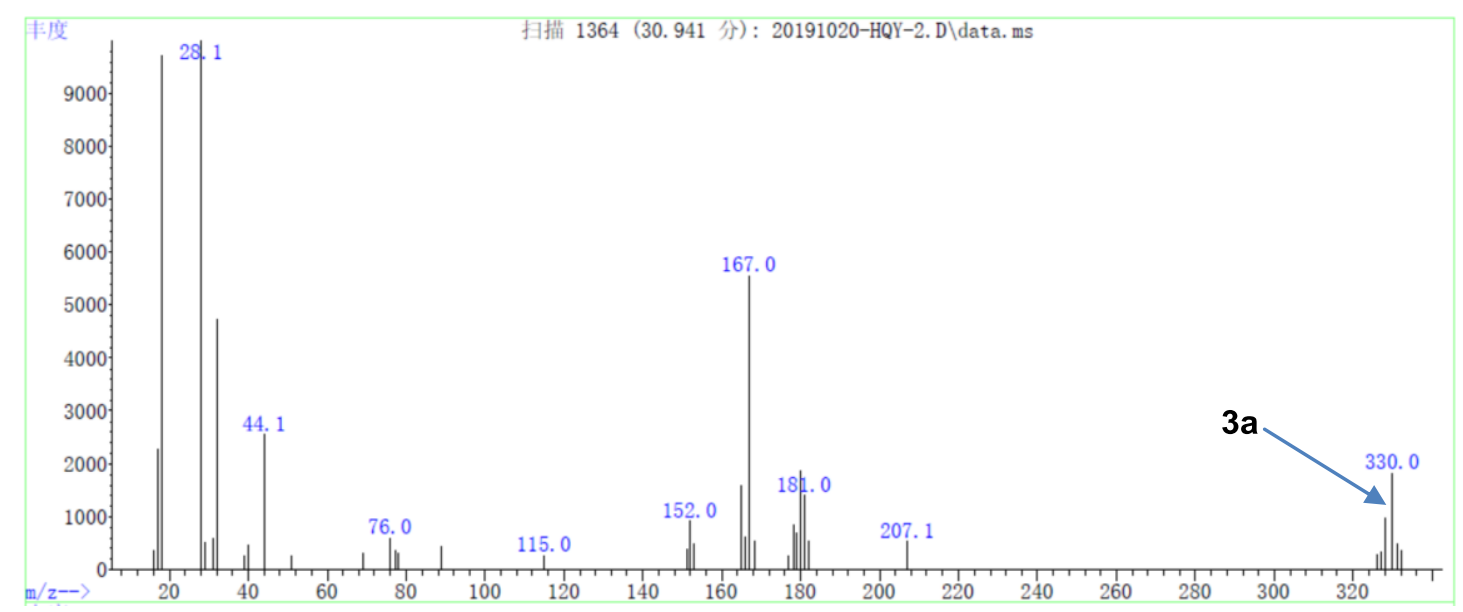


4.5. ${ }^{19} \mathrm{~F}$ NMR analysis of a reaction mixture of $1 \mathrm{a}, \mathrm{NFSI},\left[\mathrm{Me}_{4} \mathrm{~N}\right]\left[\mathrm{SeCF}_{3}\right]$, and $\mathrm{CH}_{3} \mathrm{CN}$ at room temperature for $1 \mathrm{~h}$ without photocatalyst and light irradiation.

Procedure: In a nitrogen-filled glovebox, a sealed tube was charged with 1a $(0.26$ mmol), NFSI (0.26 mmol), $\left[\mathrm{Me}_{4} \mathrm{~N}\right]\left[\mathrm{SeCF}_{3}\right](0.20 \mathrm{mmol})$, and $\mathrm{CH}_{3} \mathrm{CN}(2 \mathrm{~mL})$ with vigorous stirring. The mixture was maintained at room temperature without blue LEDs irradiation for $1 \mathrm{~h}$ and analyzed by ${ }^{19} \mathrm{~F}$ NMR spectroscopy using $\mathrm{PhOCF}_{3}(26.4$ $\mathrm{mg}, 0.163 \mathrm{mmol}$ ) as an internal standard.

Figure 10. The ${ }^{19}$ F NMR spectrum of the above reaction mixture.

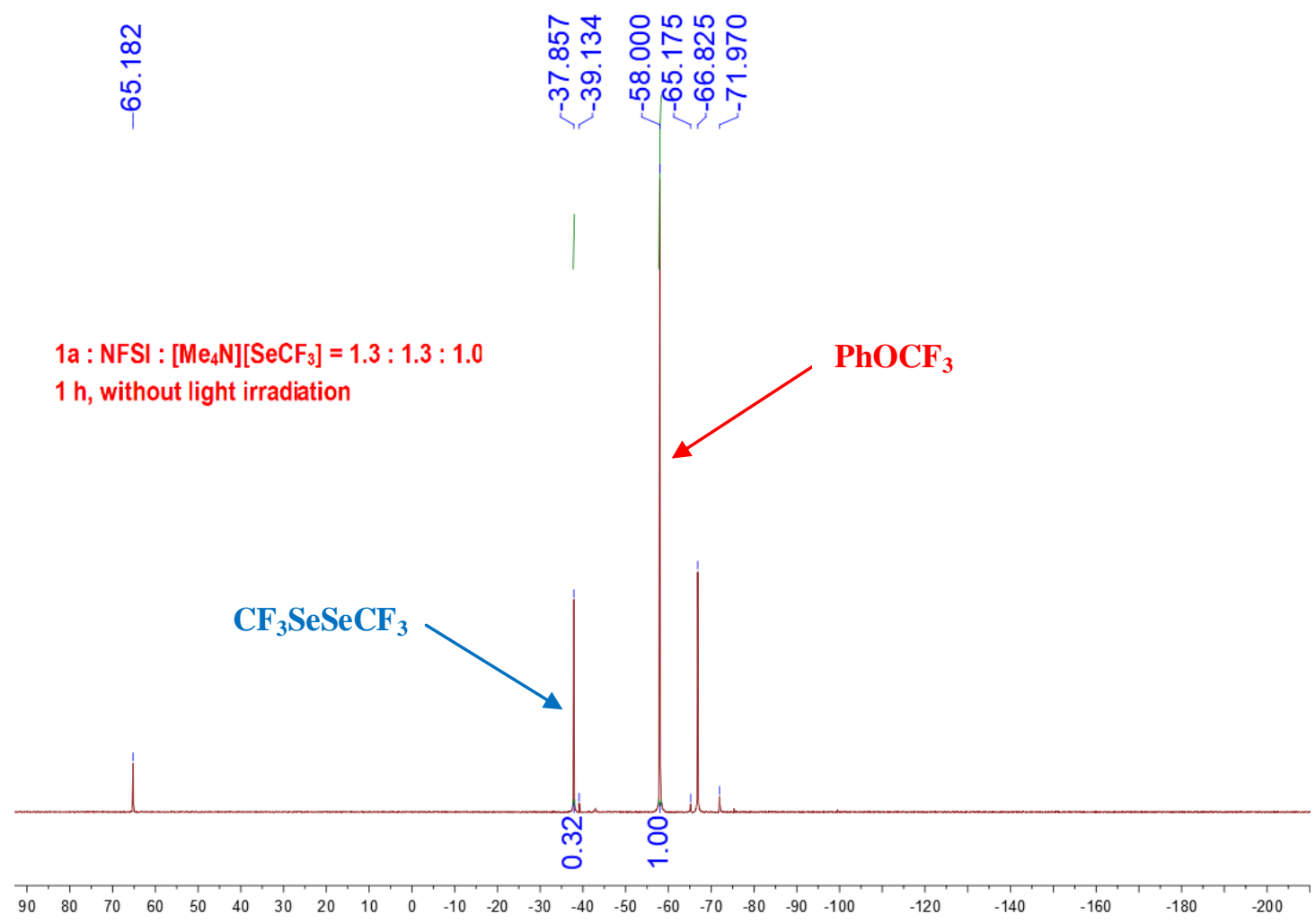

4.6. ${ }^{19} \mathrm{~F}$ NMR analysis of a mixture of NFSI $(0.26 \mathrm{mmol}),\left[\mathrm{Me}_{4} \mathrm{~N}\right]\left[\mathrm{SeCF}_{3}\right](0.20$ mmol) and $\mathrm{CH}_{3} \mathrm{CN}(2 \mathrm{~mL})$ at room temperature for $1 \mathrm{~h}\left(27.6 \mathrm{mg}\right.$ of $\mathrm{PhOCF}_{3}$ (0.170 mmol) was used as an internal standard).

Figure 11 
$\stackrel{\infty}{\infty}$

เి

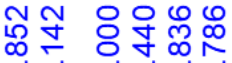

กิ

$\mathrm{NFSI}:\left[\mathrm{Me}_{4} \mathrm{~N}\right]\left[\mathrm{SeCF}_{3}\right]=1.3: 1.0$

$1 \mathrm{~h}$, without light irradiation

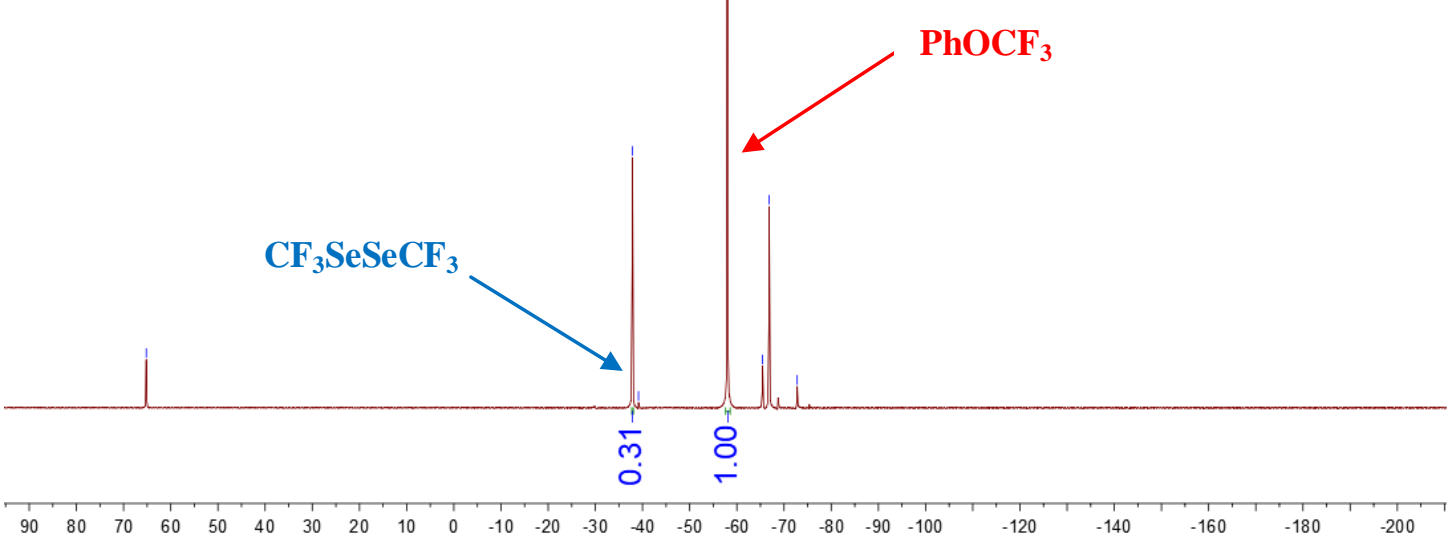

4.7. ${ }^{19} \mathrm{~F}$ NMR analysis of a mixture of $\left(\mathrm{PhSO}_{2}\right)_{2} \mathrm{NH}(0.20 \mathrm{mmol}),\left[\mathrm{Me}_{4} \mathrm{~N}\right]\left[\mathrm{SeCF}_{3}\right]$ $(0.20 \mathrm{mmol})$ and $\mathrm{CH}_{3} \mathrm{CN}(2 \mathrm{~mL})$ at room temperature for $1 \mathrm{~h}\left(27.5 \mathrm{mg}\right.$ of $\mathrm{PhOCF}_{3}$ (0.170 mmol) was used as an internal standard).

\section{Figure 12}

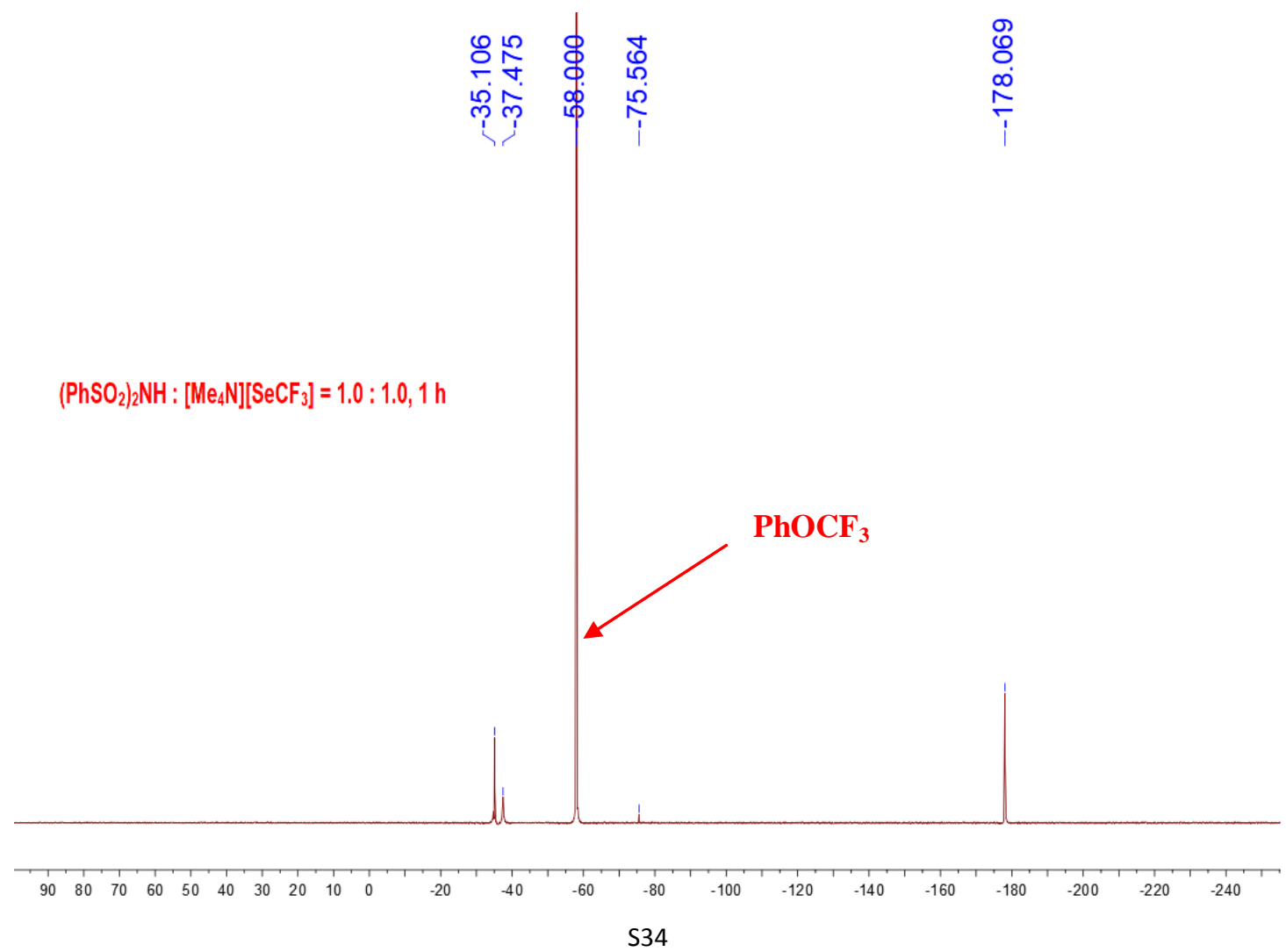


4.8. The ${ }^{19} \mathrm{~F}$ NMR spectrum of NFSI $(0.26 \mathrm{mmol})$ in $\mathrm{CH}_{3} \mathrm{CN}(2 \mathrm{~mL})$ using $\mathrm{PhOCF}_{3}(27.5 \mathrm{mg}, 0.170 \mathrm{mmol})$ as an internal standard.

Figure 13

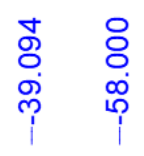

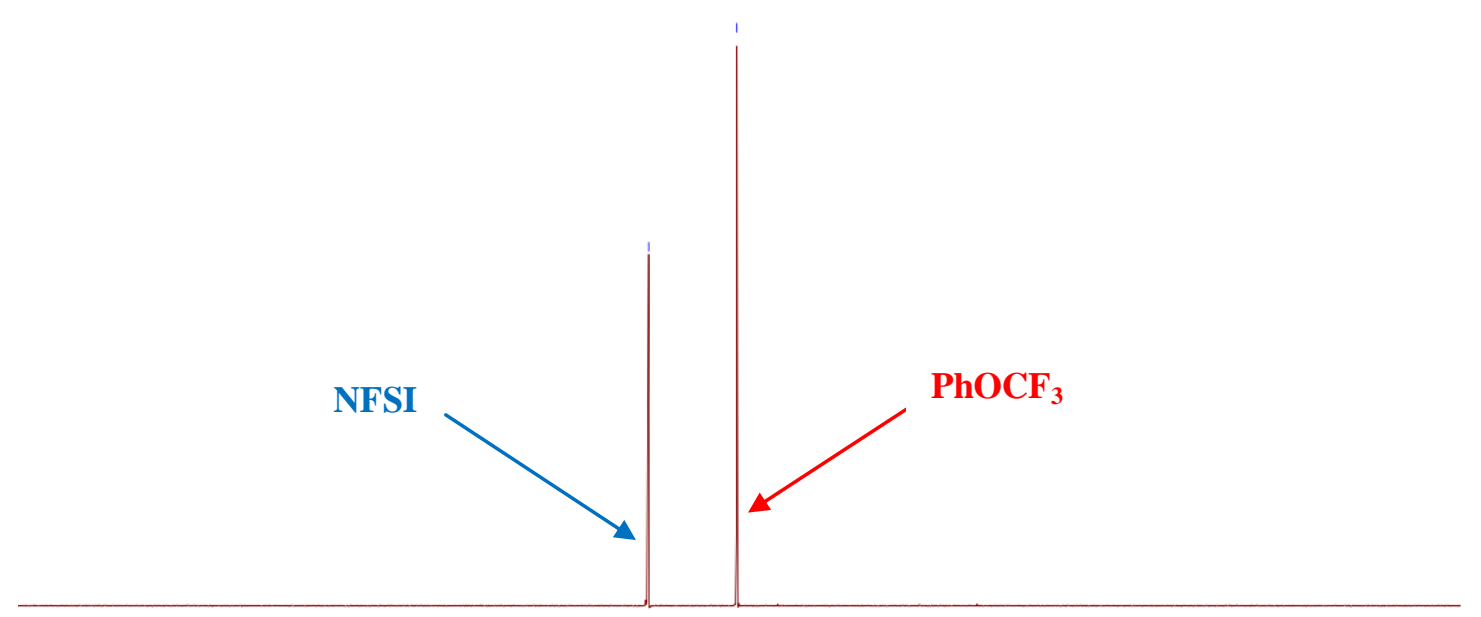

$\begin{array}{rllllllllllllllllllllllllll}1 & 80 & 70 & 60 & 50 & 40 & 30 & 20 & 10 & 0 & -10 & -20 & -30 & -40 & -50 & -60 & -70 & -80 & -90 & -100 & -120 & -140 & -160 & -180 & -200\end{array}$

4.9. HPLC analysis of the side products in the standard reaction of 1a, NFSI, $\left[\mathrm{Me}_{4} \mathrm{~N}\right]\left[\mathrm{SeCF}_{3}\right]$ and $[\mathrm{Mes}-\mathrm{Acr}-\mathrm{Ph}]\left[\mathrm{BF}_{4}\right]$.

Procedure: In a nitrogen-filled glovebox, a sealed tube was charged with 1a $(0.26$ $\mathrm{mmol}), \mathrm{NFSI}(0.26 \mathrm{mmol}),\left[\mathrm{Me}_{4} \mathrm{~N}\right]\left[\mathrm{SeCF}_{3}\right](0.20 \mathrm{mmol}),[\mathrm{Mes}-\mathrm{Acr}-\mathrm{Ph}]\left[\mathrm{BF}_{4}\right](0.002$ $\mathrm{mmol}$ ) and $\mathrm{CH}_{3} \mathrm{CN}(2 \mathrm{~mL})$ with vigorous stirring. The mixture was irradiated by $2 \times$ $10 \mathrm{~W}$ blue LEDs ( $7 \mathrm{~cm}$ away) at room temperature for $24 \mathrm{~h}$ and analyzed by HPLC $\left(\lambda_{\max }=220 \mathrm{~nm}\right.$, water/acetonitrile $\left.(\mathrm{v} / \mathrm{v})=20: 80\right)$ using $\left(\mathrm{PhSO}_{2}\right)_{2} \mathrm{NH}\left(\mathrm{t}_{\mathrm{R}}=1.92 \mathrm{~min}\right)$, $\mathrm{PhSO}_{2} \mathrm{NH}_{2}\left(\mathrm{t}_{\mathrm{R}}=3.23 \mathrm{~min}\right), \mathrm{PhSO}_{2} \mathrm{~F}\left(\mathrm{t}_{\mathrm{R}}=4.28 \mathrm{~min}\right)$, and $\mathbf{3 a}\left(\mathrm{t}_{\mathrm{R}}=11.38 \mathrm{~min}\right)$ as external standards, respectively. The results showed that in addition to $\mathbf{3 a}$ (the desired product), the side products $\left(\mathrm{PhSO}_{2}\right)_{2} \mathrm{NH}(37 \%), \mathrm{PhSO}_{2} \mathrm{NH}_{2}(44 \%)$, and $\mathrm{PhSO}_{2} \mathrm{~F}(58 \%)$ were formed. The production of $\mathrm{PhSO}_{2} \mathrm{~F}$ and $\mathrm{PhSO}_{2} \mathrm{NH}_{2}$ was also evidenced by the ${ }^{19}$ F NMR and/or GC-MS analyses of the reaction mixtures (e.g., Figures 1-6 and 9). 


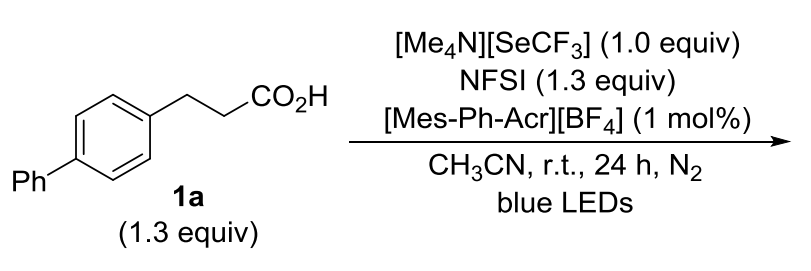

blue LEDs

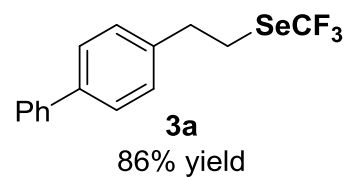

(based on $\left[\mathrm{Me}_{4} \mathrm{~N}\right]\left[\mathrm{SeCF}_{3}\right]$ )

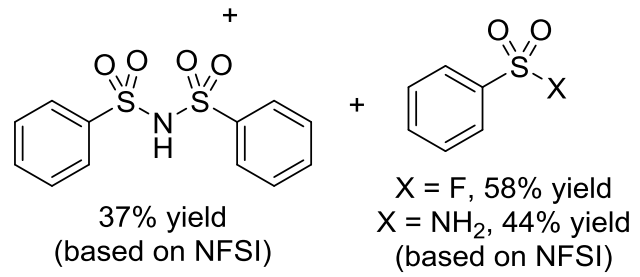

4.10. ${ }^{19}$ F NMR analysis of a reaction mixture of $1 \mathrm{a}(0.26 \mathrm{mmol})$, NFSI $(0.26 \mathrm{mmol})$ [Mes-Acr-Ph] $\left[\mathrm{BF}_{4}\right](0.002 \mathrm{mmol})$ and $\mathrm{CH}_{3} \mathrm{CN}(2 \mathrm{~mL})$ in the absence of $\left[\mathrm{Me}_{4} \mathrm{~N}\right]\left[\mathrm{SeCF}_{3}\right]$ at room temperature under the standard conditions for $24 \mathrm{~h}$ ( PhOCF $_{3}$ was used as an internal standard).

\section{Figure 14}

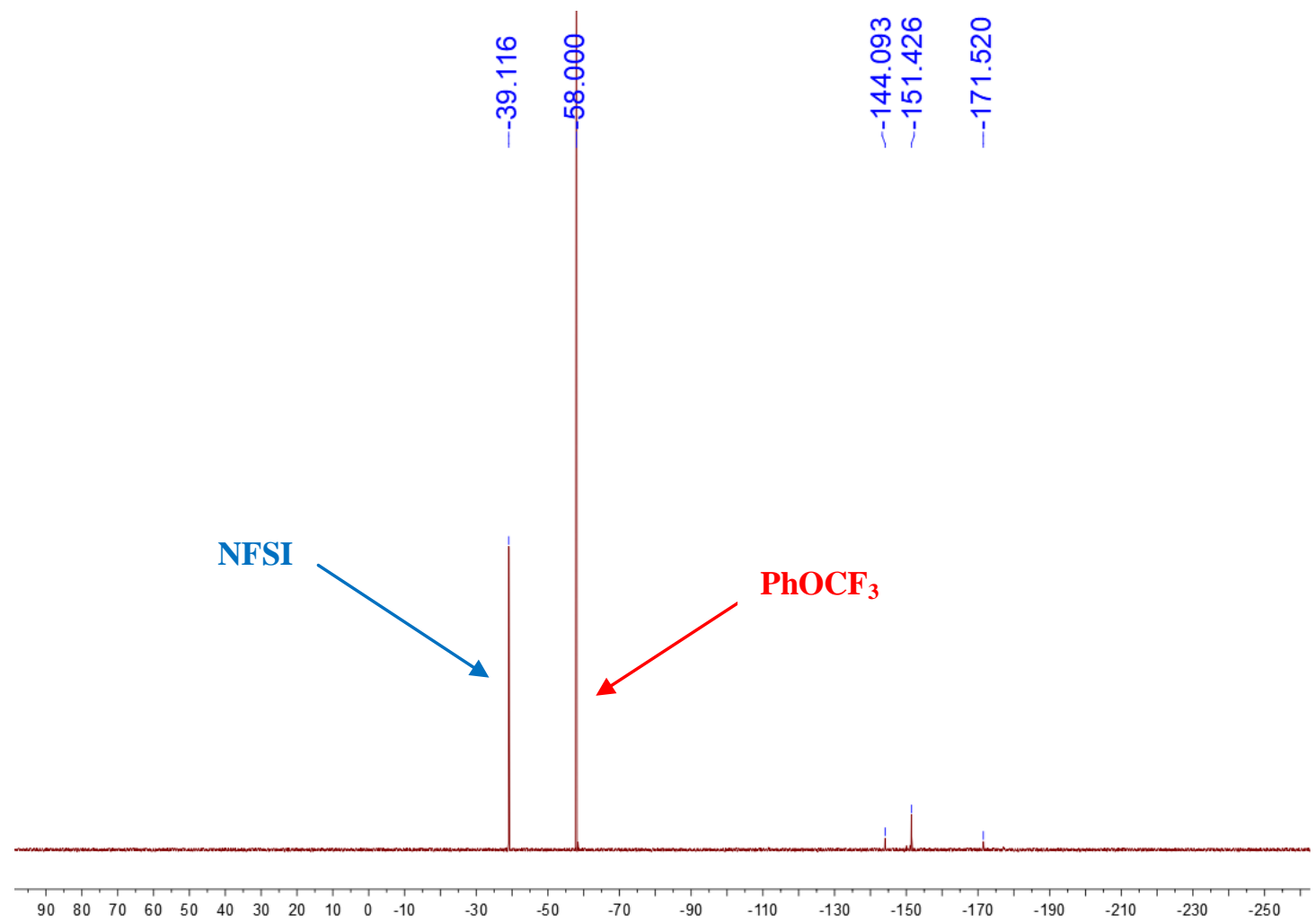

\subsection{The EPR experiments}

EPR assays were carried out at room temperature using a Bruker EMX spectrometer operating at X-band with $9.8 \mathrm{GHz}$ modulation frequency. The instrument settings were as follows: microwave power: $2 \mathrm{~mW}$; modulation amplitude: $1 \mathrm{G}$; time constant: 
30 ms; number of scans: 5 .

Procedure A: In a nitrogen-filled glovebox, a sealed tube was charged with 3-([1,1'-biphenyl]-4-yl)propanoic acid (1a, 0.13 mmol), PBN (0.23 mmol), $\left[\mathrm{Me}_{4} \mathrm{~N}\right]\left[\mathrm{SeCF}_{3}\right](0.10 \mathrm{mmol}),[\mathrm{Mes}-\mathrm{Acr}-\mathrm{Ph}]\left[\mathrm{BF}_{4}\right](0.001 \mathrm{mmol}), \mathrm{NFSI}(0.13 \mathrm{mmol})$, and $\mathrm{CH}_{3} \mathrm{CN}$ (1.5 mL) with vigorous stirring. The mixture was irradiated by $2 \times 10 \mathrm{~W}$ blue LEDs ( $7 \mathrm{~cm}$ away) at room temperature for $7 \mathrm{~min}$ and then analyzed by EPR spectroscopy.

Figure 15. The EPR spectrum of the above reaction mixture.

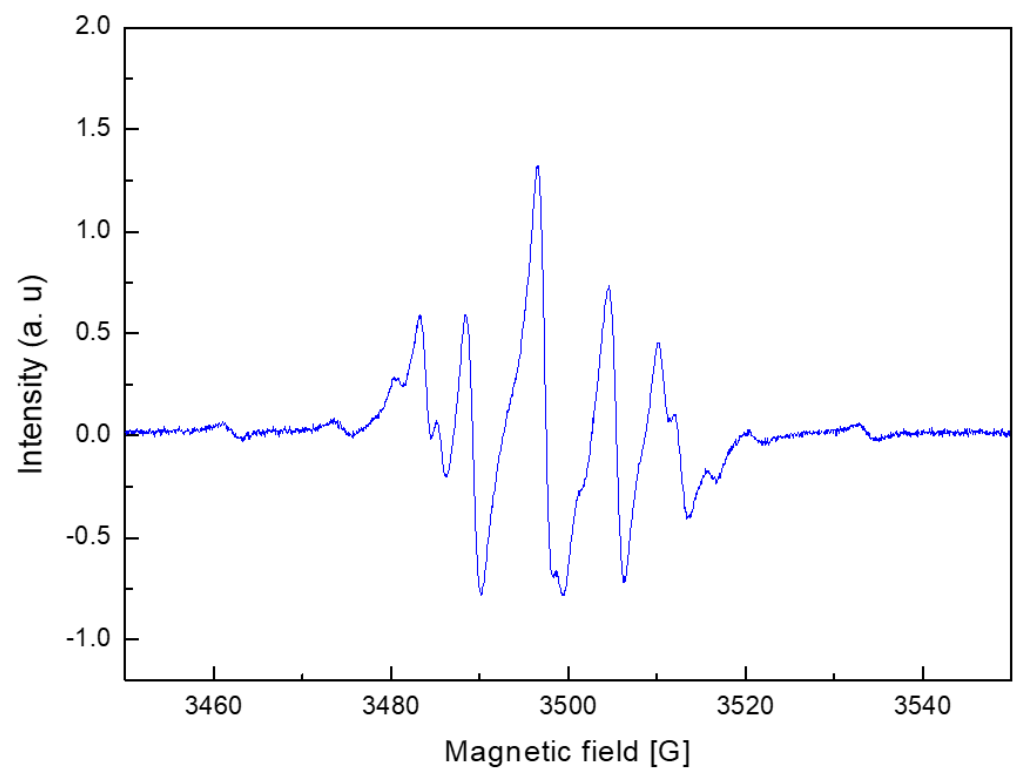

Procedure B: In a nitrogen-filled glovebox, a sealed tube was charged with 3-([1,1'-biphenyl]-4-yl)propanoic acid (1a, 0.10 mmol), PBN $(0.10$ mmol$)$, [Mes-Acr-Ph] $\left[\mathrm{BF}_{4}\right](0.001 \mathrm{mmol}), \mathrm{NFSI}(0.05 \mathrm{mmol})$, and $\mathrm{CH}_{3} \mathrm{CN}(1.5 \mathrm{~mL})$ with vigorous stirring. The mixture was irradiated by $2 \times 10 \mathrm{~W}$ blue LEDs $(7 \mathrm{~cm}$ away) at room temperature for 7 min and then analyzed by EPR spectroscopy.

Figure 16. The EPR spectrum of the above reaction mixture. 


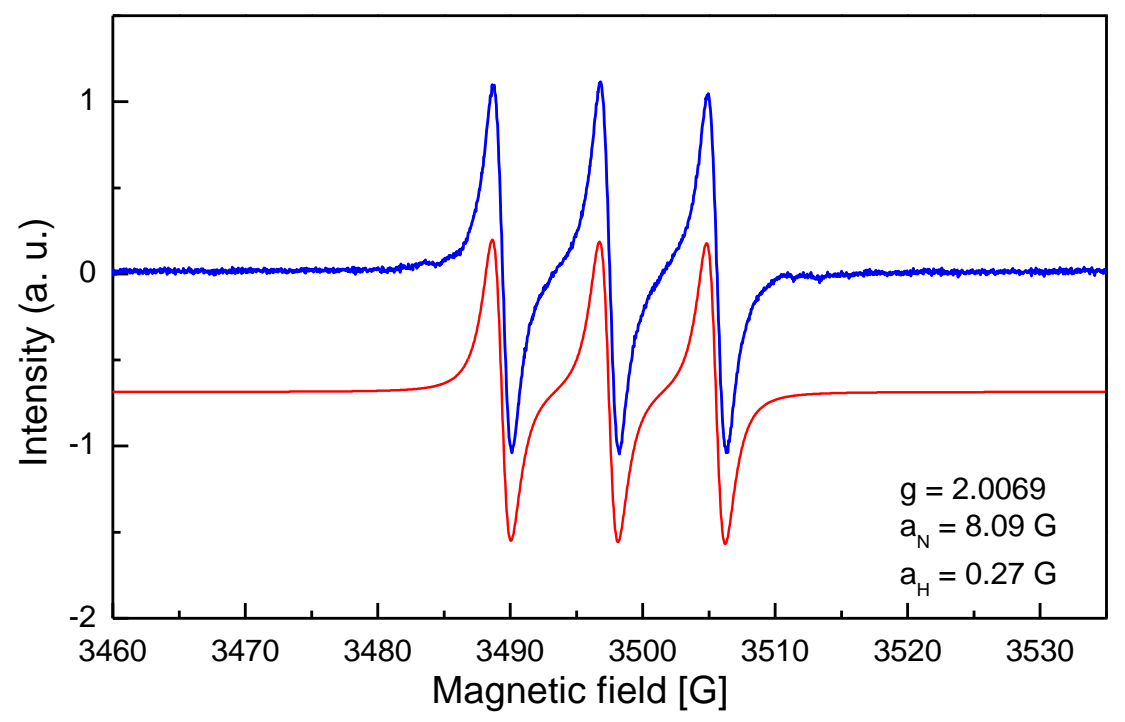

\section{References}

[1] Tyrra, W.; Kremer, S. Synthesis of Zero-Valent Trifluoromethyl Chalcogenato Derivatives, $\left[\mathrm{NMe}_{4}\right] \mathrm{ECF}_{3}(\mathrm{E}=\mathrm{S}, \mathrm{Se}, \mathrm{Te})$, and Related Compounds (Chapter 8), Efficient Preparations of Fluorine Compounds, $1^{\text {st }}$ edition (by H. W. Roesky), 2013, John Wiley \& Sons, Inc.

[2] Wilger, D. J.; Grandjean, J.-M. M.; Lammert, T. R.; Nicewicz. D. A. Nat. Chem. 2014, 6, 720-726.

[3] Armarego, W. L. F.; Chai, C. L. L. Purification of Laboratory Chemicals, $5^{\text {th }}$ ed; Butterworth Heinemann: Oxford, 2003.

[4] (a) Dong, T.; He, J.; Li, Z.-H.; Zhang, C.-P. ACS Sustainable Chem. Eng. 2018, 6, 1327-1335. (b) Chen, X.-L.; Zhou, S.-H.; Lin, J.-H.; Deng, Q.-H.; Xiao, J.-C. Chem. Commun. 2019, 55, 1410-1413. (c) Feldhoff, R.; Haas, A.; Lieb, M. J. Fluorine Chem. 1994, 67, 245-251 
5. The NMR spectra of the products (3)

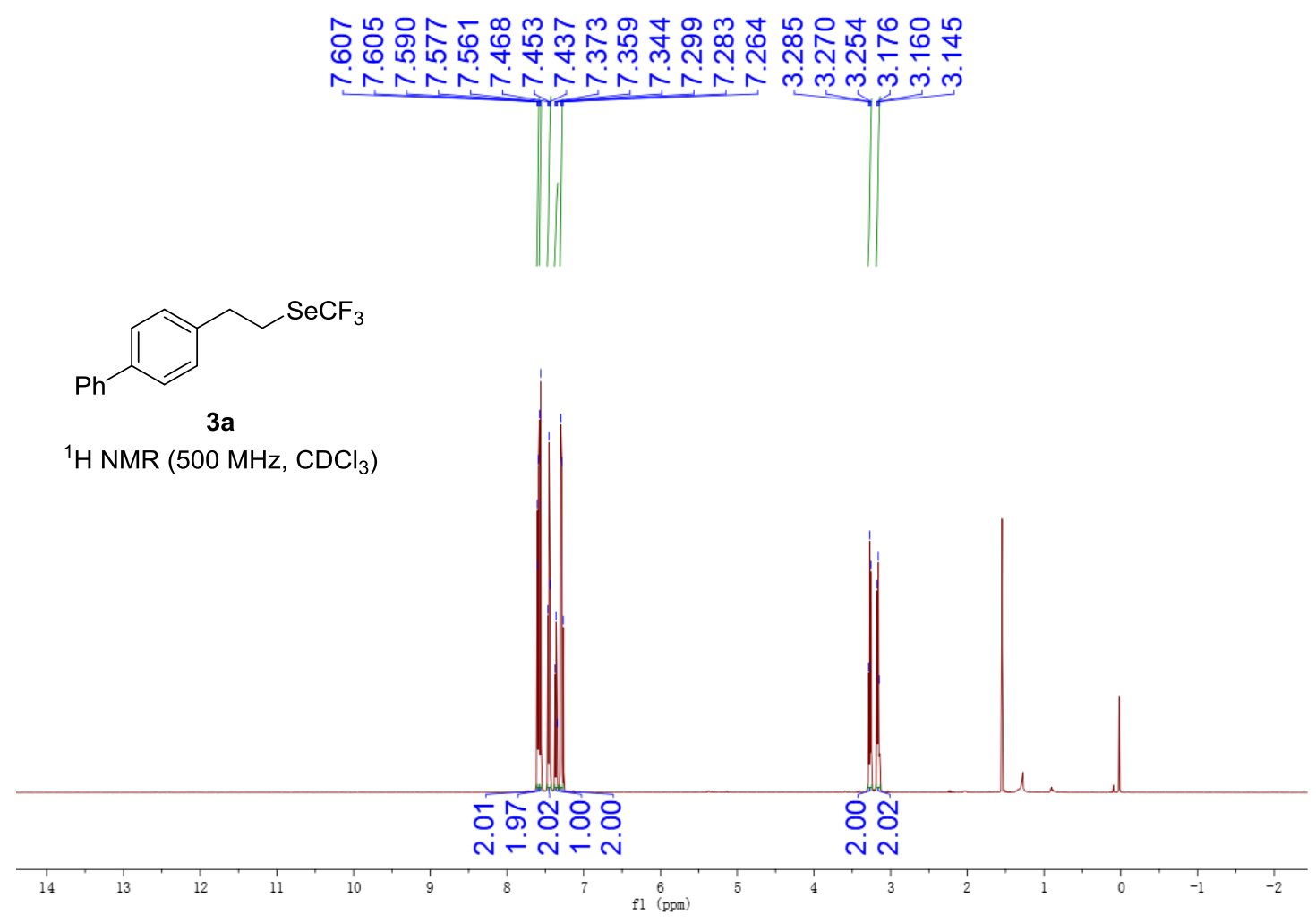

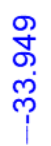

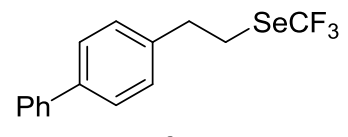

$3 a$

${ }^{19} \mathrm{~F}$ NMR $\left(471 \mathrm{MHz}, \mathrm{CDCl}_{3}\right)$ 

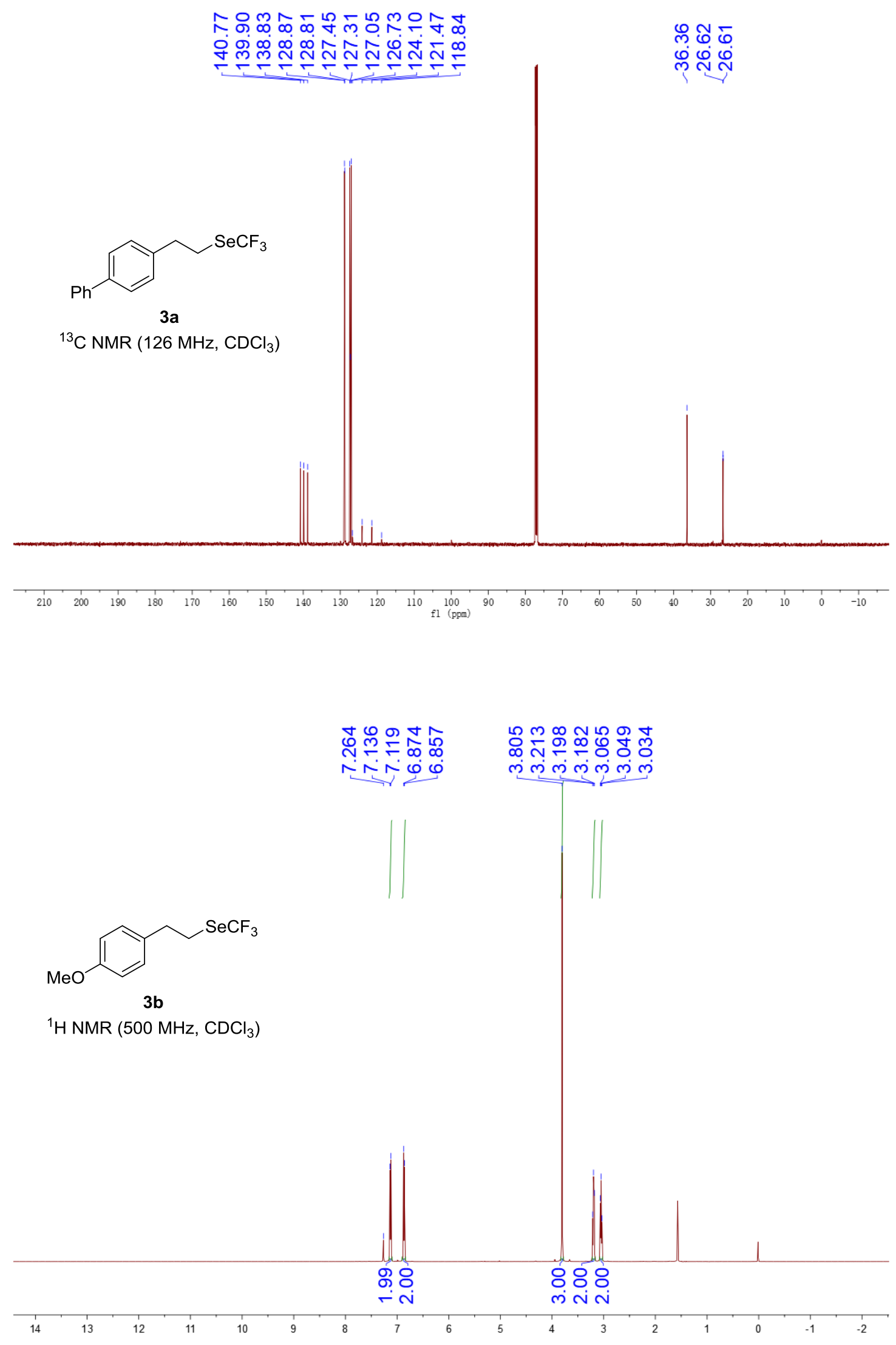


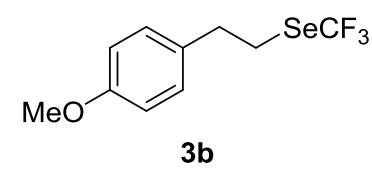

${ }^{19} \mathrm{~F} \mathrm{NMR}\left(471 \mathrm{MHz}, \mathrm{CDCl}_{3}\right)$

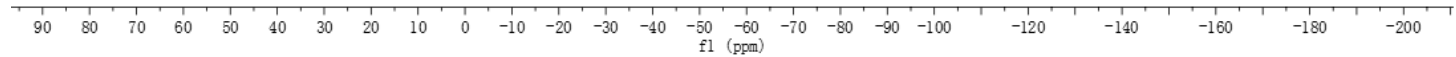

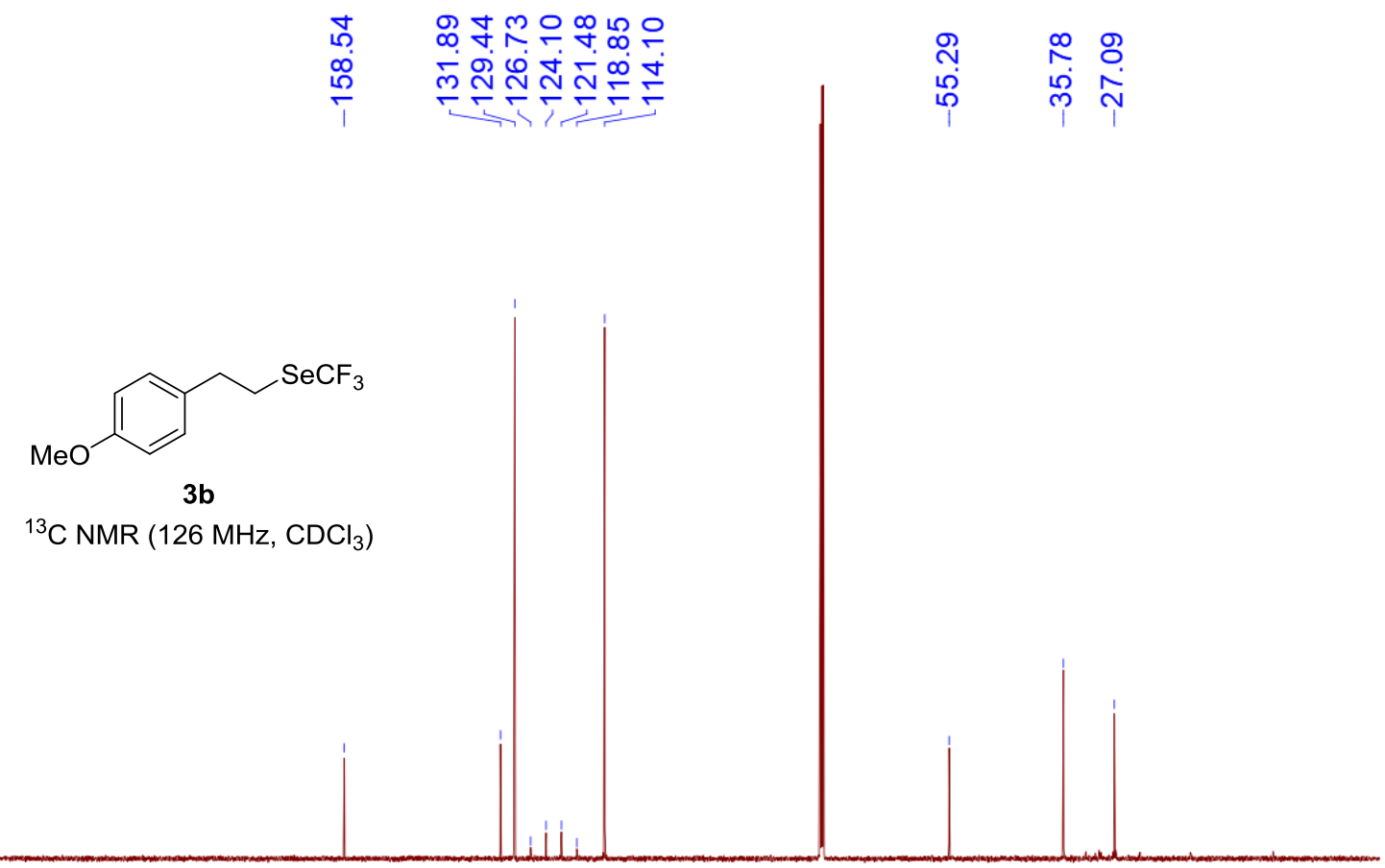

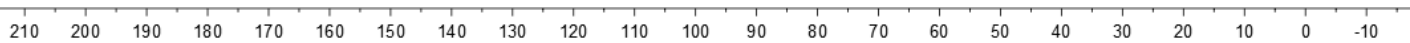




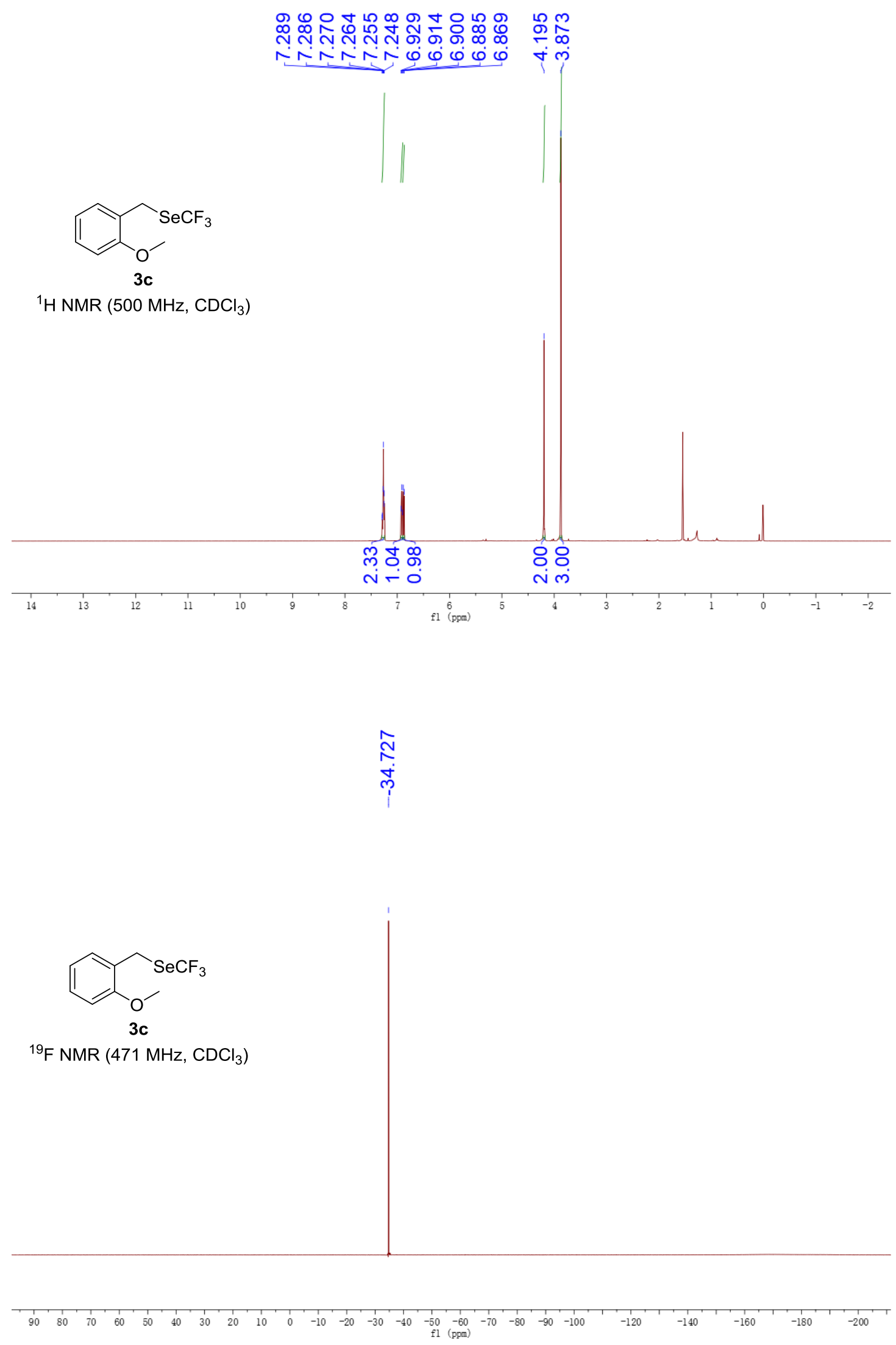



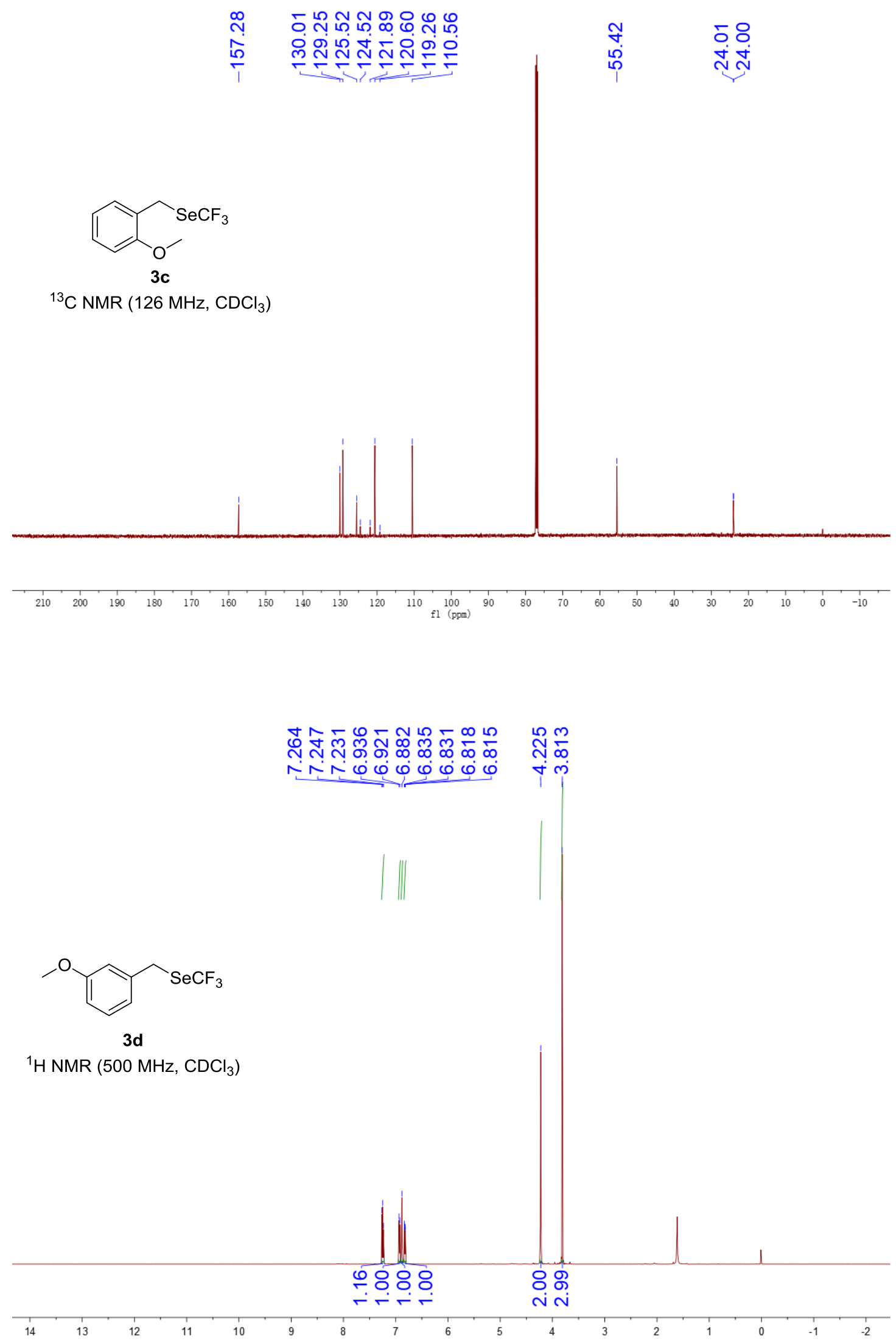


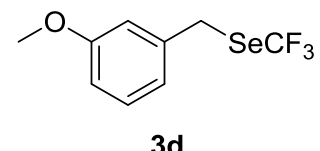

${ }^{19} \mathrm{~F} \mathrm{NMR}\left(471 \mathrm{MHz}, \mathrm{CDCl}_{3}\right)$

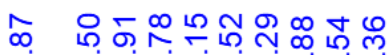

هิ

$\rightarrow \quad-5=5$

ำ

กำ

으

กั

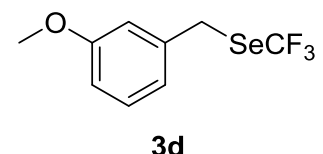

$\left.{ }^{13} \mathrm{C} \mathrm{NMR} \mathrm{(126} \mathrm{MHz,} \mathrm{CDCl}_{3}\right)$
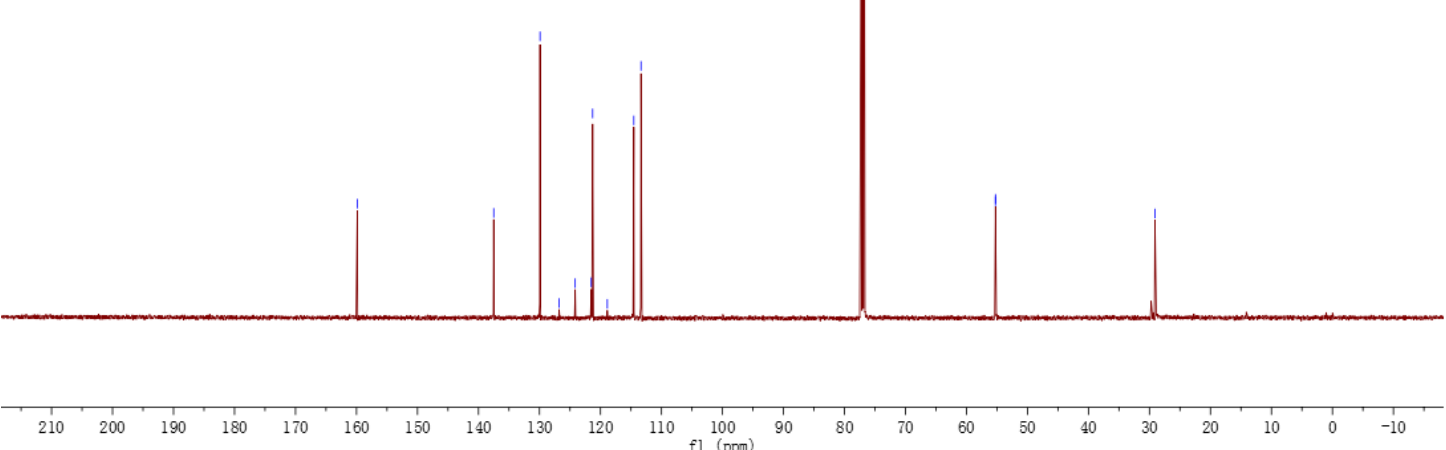


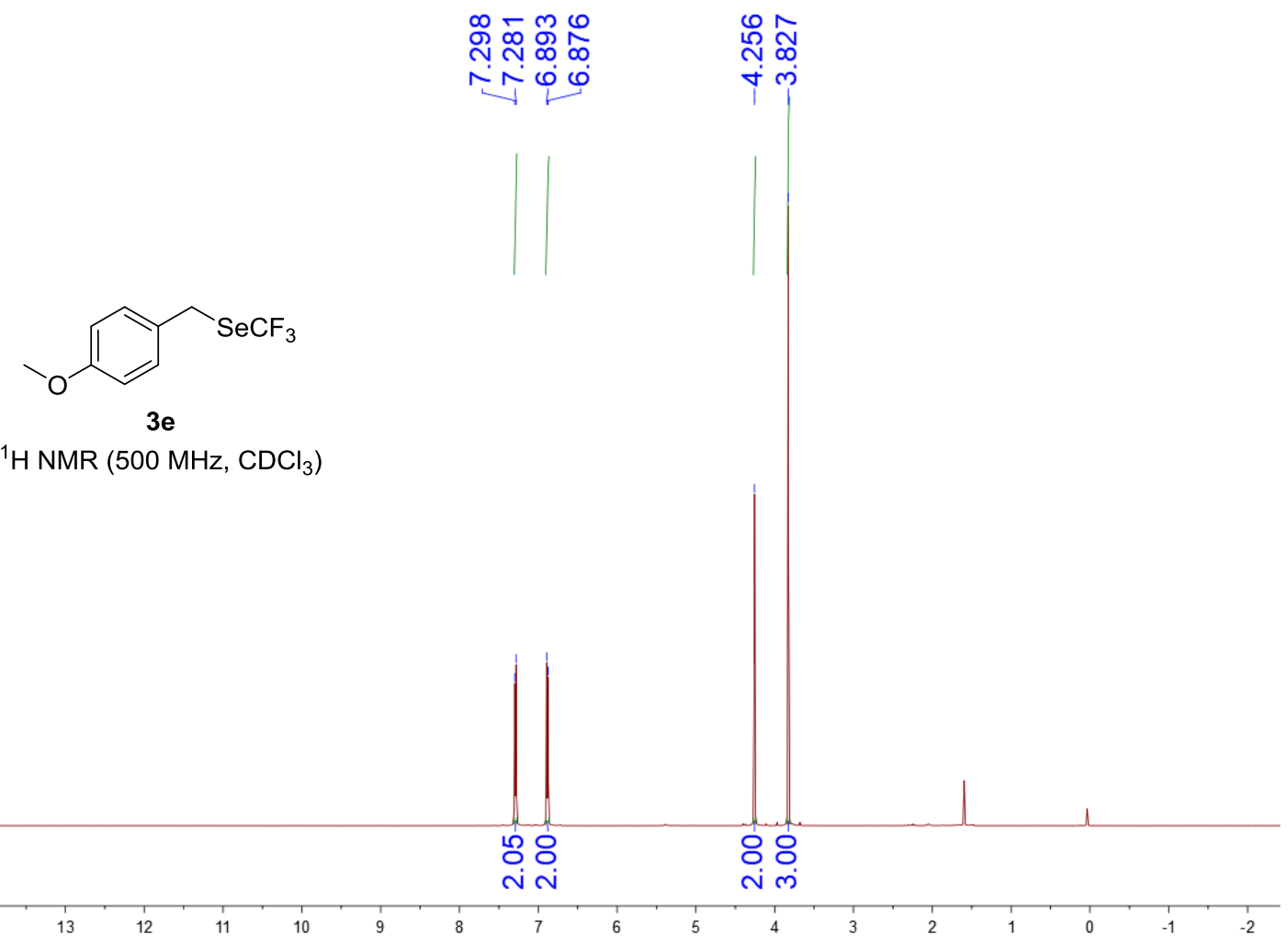

$\stackrel{+}{O}$
Ṗ

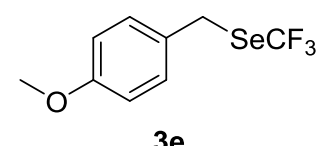

${ }^{19} \mathrm{~F} \mathrm{NMR}\left(471 \mathrm{MHz}, \mathrm{CDCl}_{3}\right)$

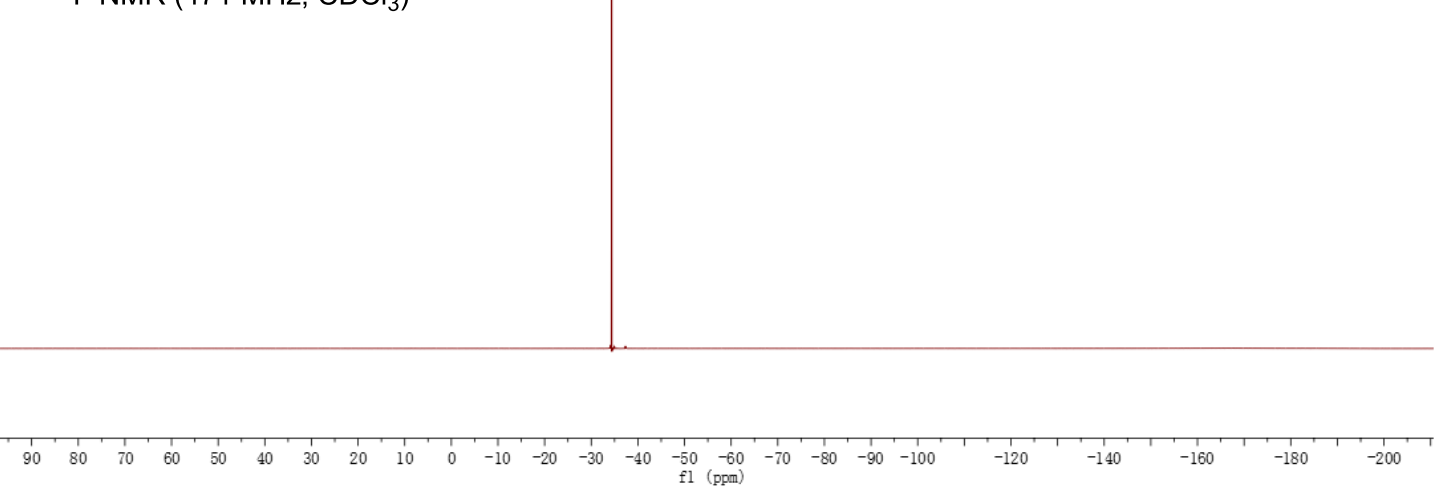



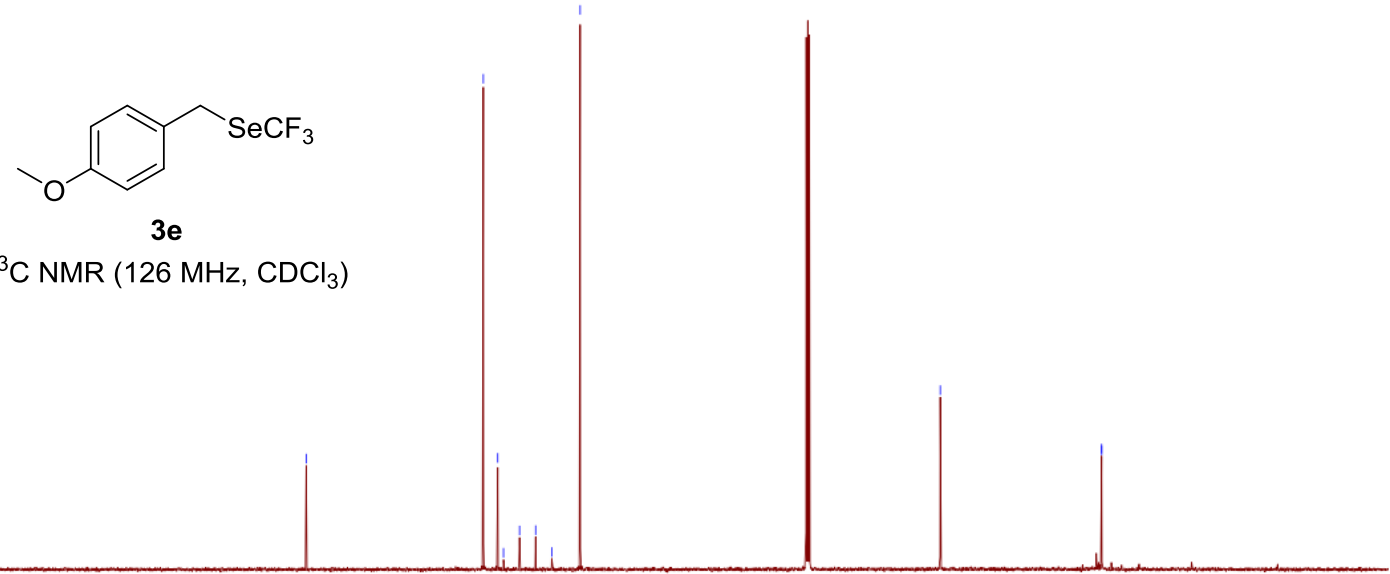

${ }^{13} \mathrm{C} \mathrm{NMR}\left(126 \mathrm{MHz}, \mathrm{CDCl}_{3}\right)$

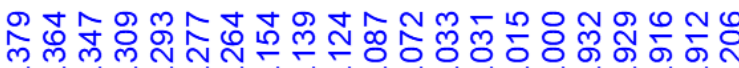

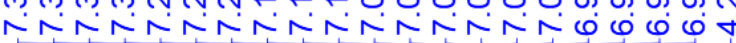

$\mathrm{SeCF}_{3}$

${ }^{1} \mathrm{H}$ NMR $\left(500 \mathrm{MHz}, \mathrm{CDCl}_{3}\right)$

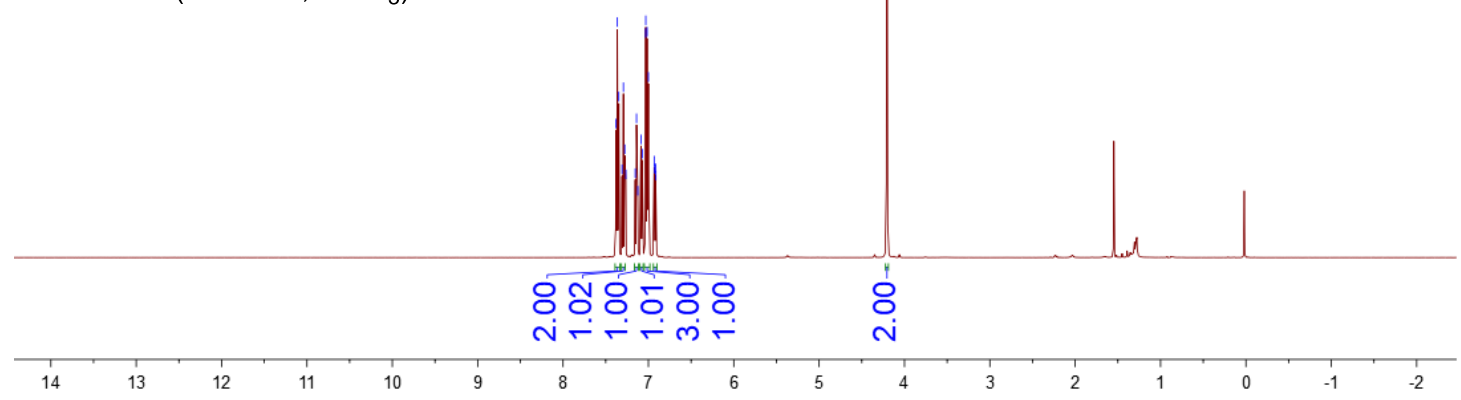




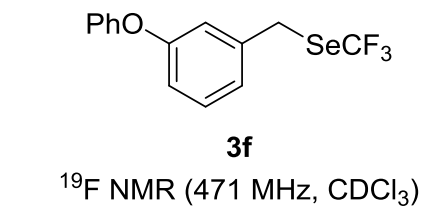

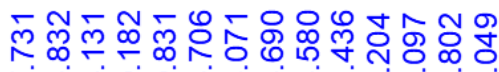

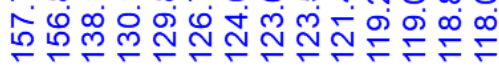

솟

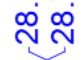

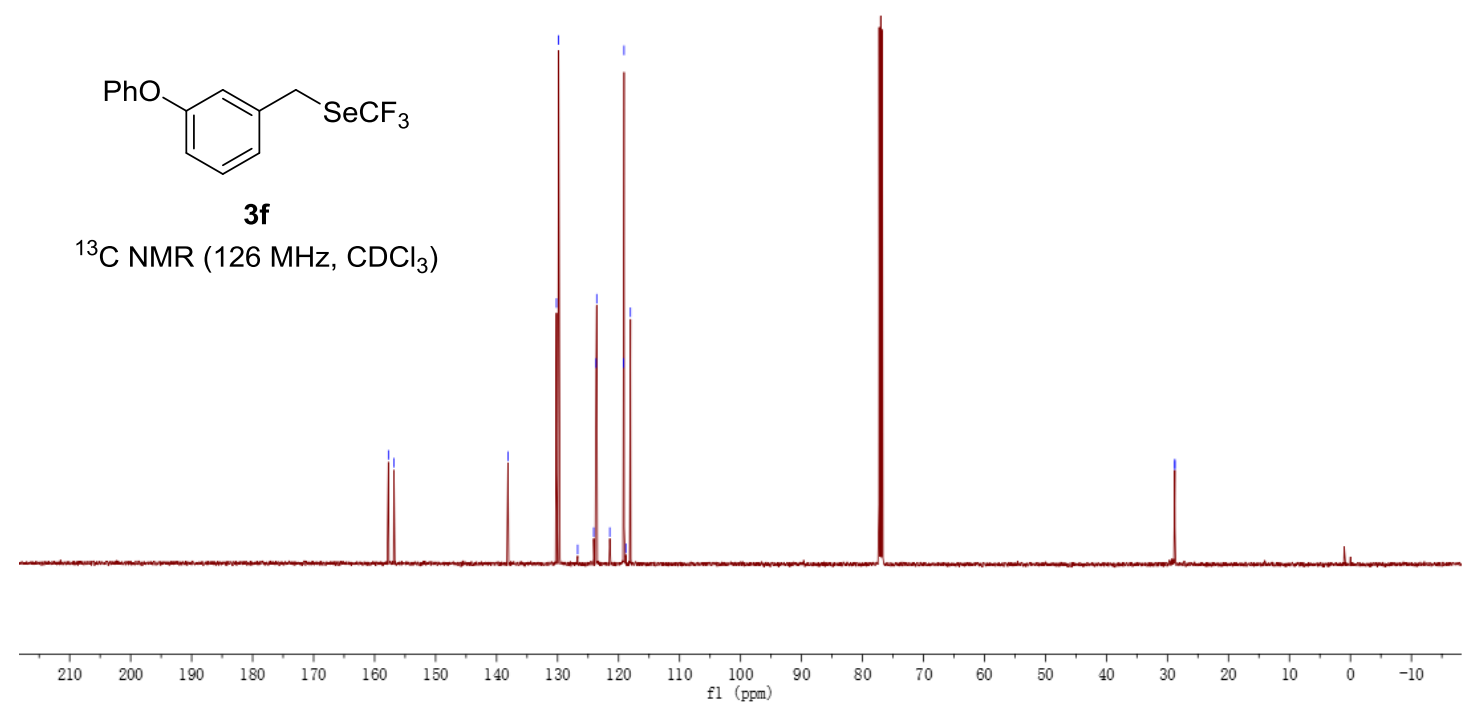




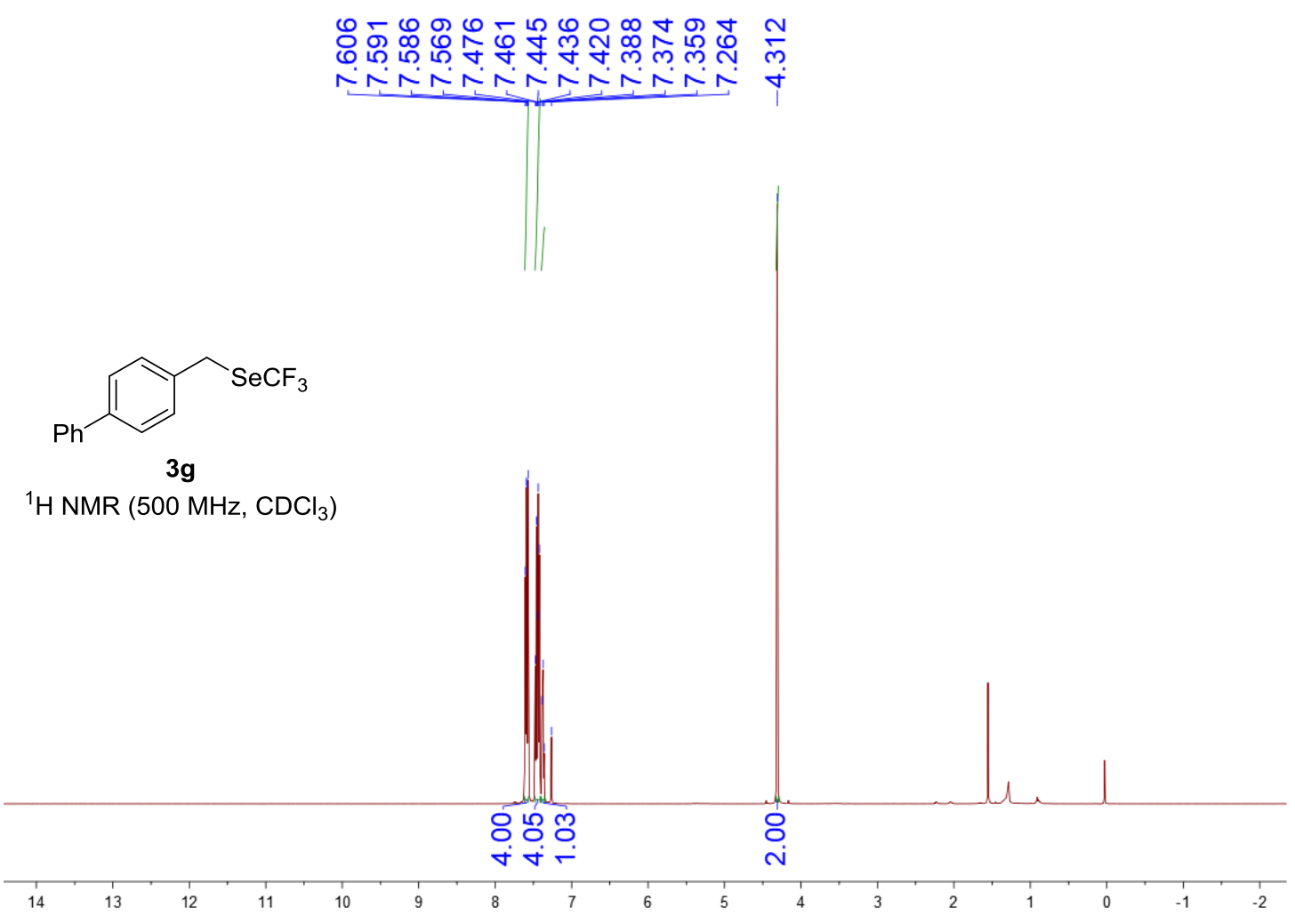

m

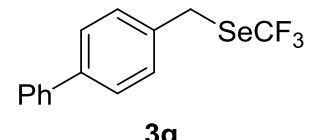

3g

${ }^{19} \mathrm{~F}$ NMR $\left(471 \mathrm{MHz}, \mathrm{CDCl}_{3}\right)$

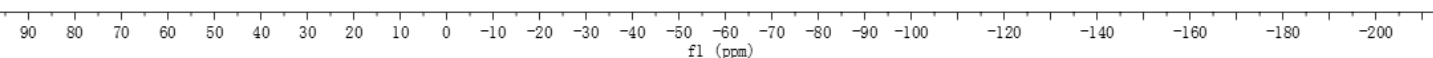




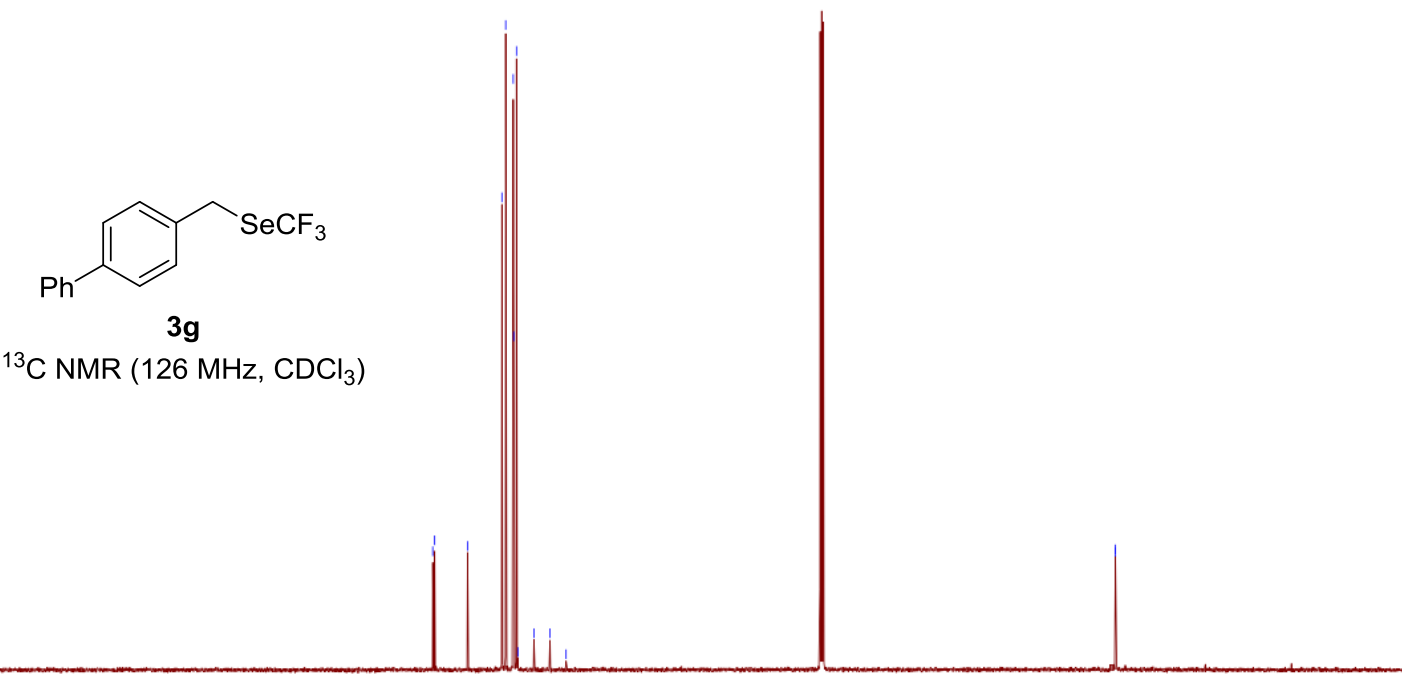

${ }^{13} \mathrm{C}$ NMR $\left(126 \mathrm{MHz}, \mathrm{CDCl}_{3}\right)$
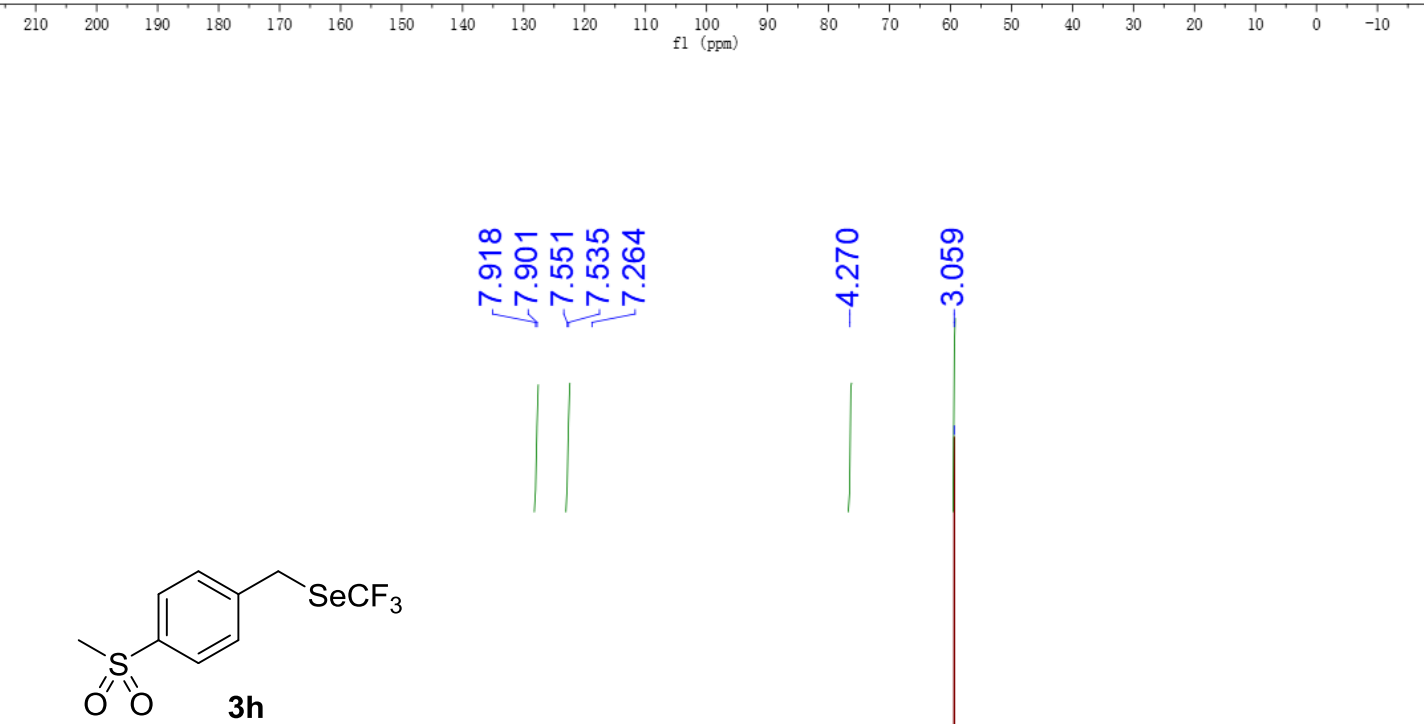

${ }^{1} \mathrm{H}$ NMR (500 MHz, $\mathrm{CDCl}_{3}$ )
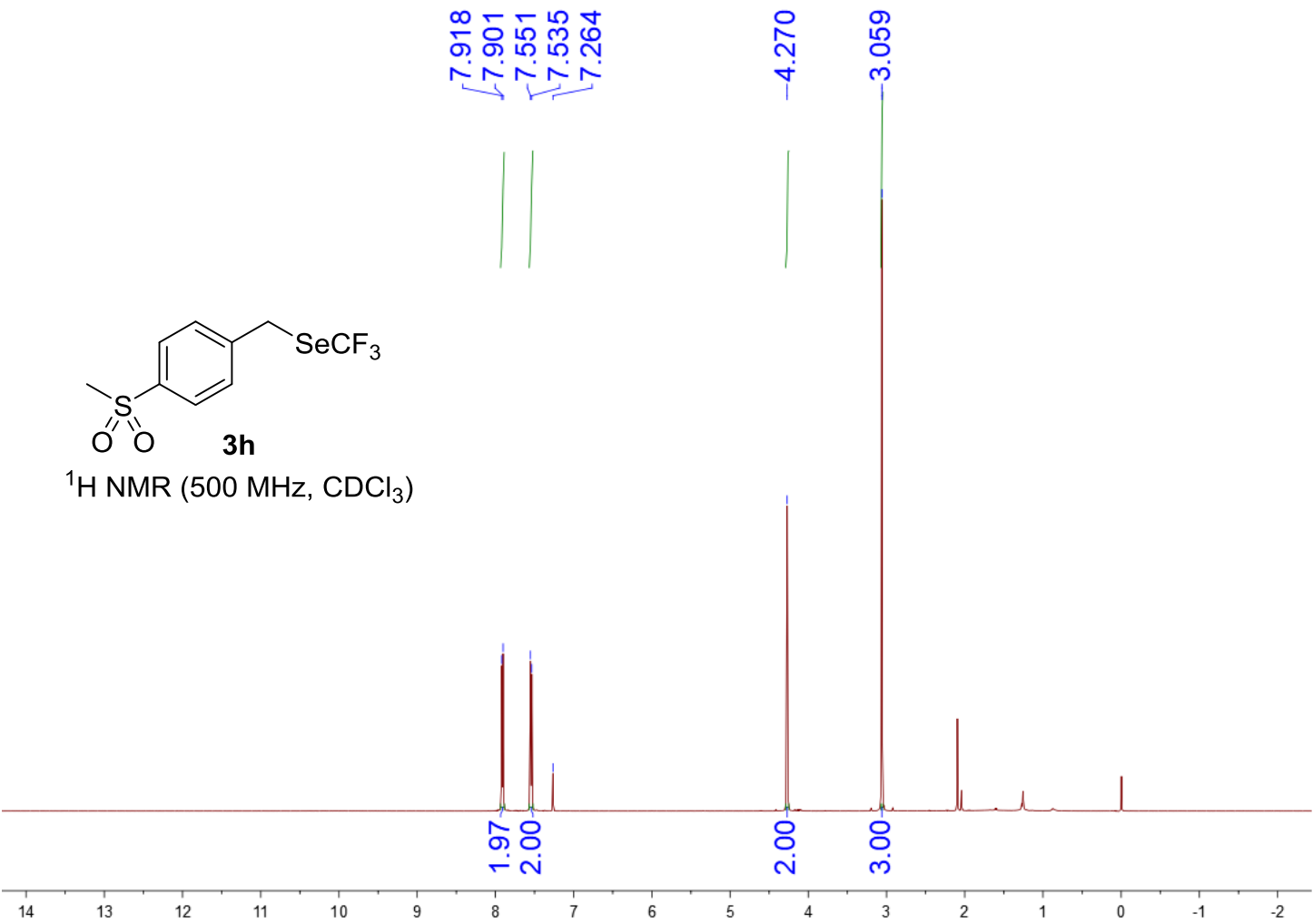


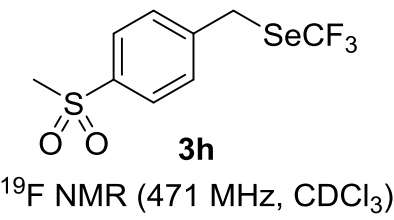

${ }^{19} \mathrm{~F}$ NMR $\left(471 \mathrm{MHz}, \mathrm{CDCl}_{3}\right)$

ㄴ.

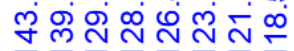

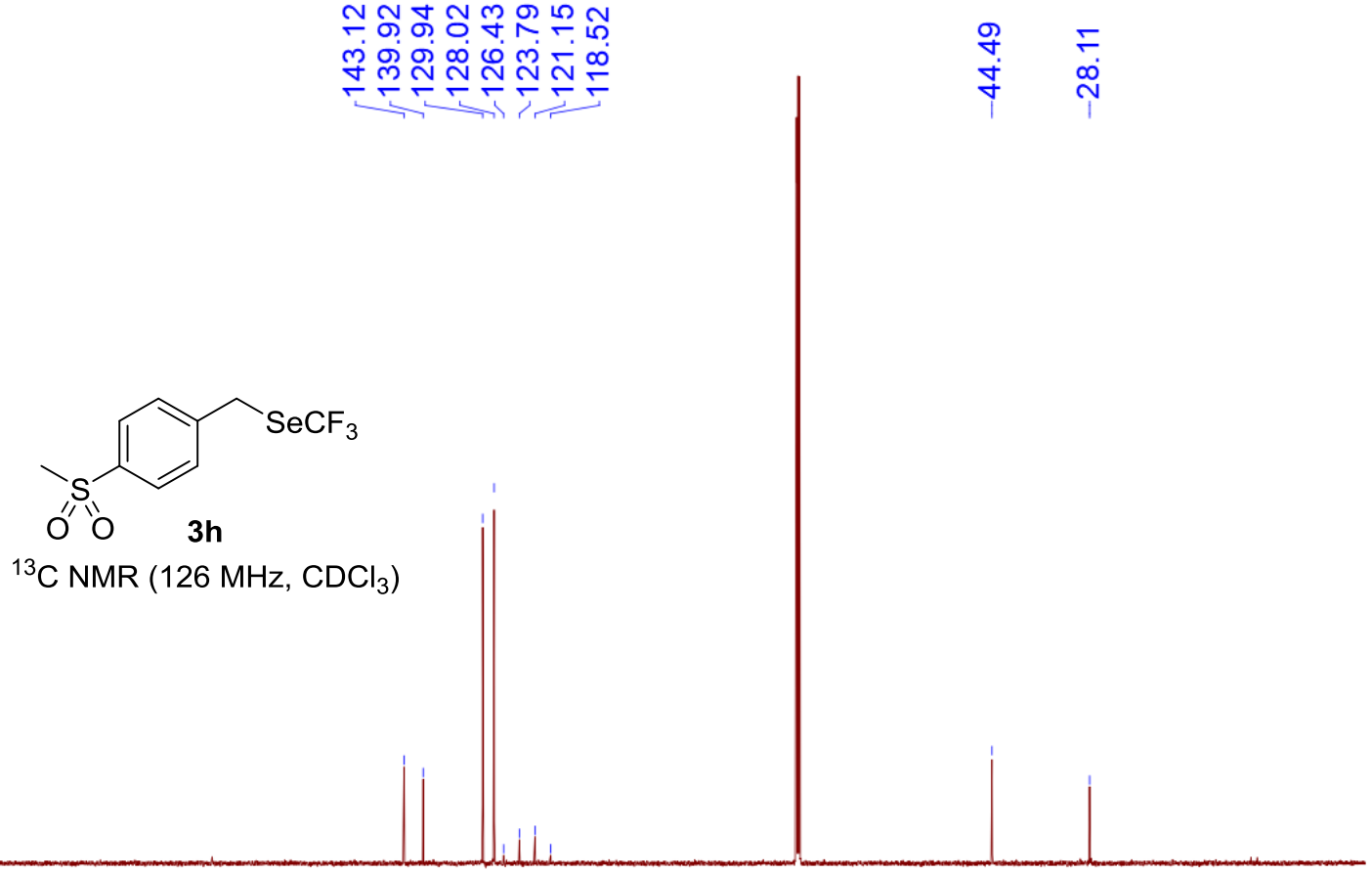

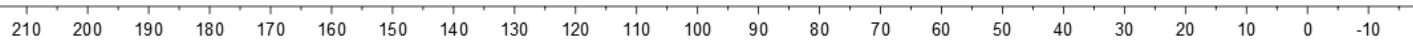




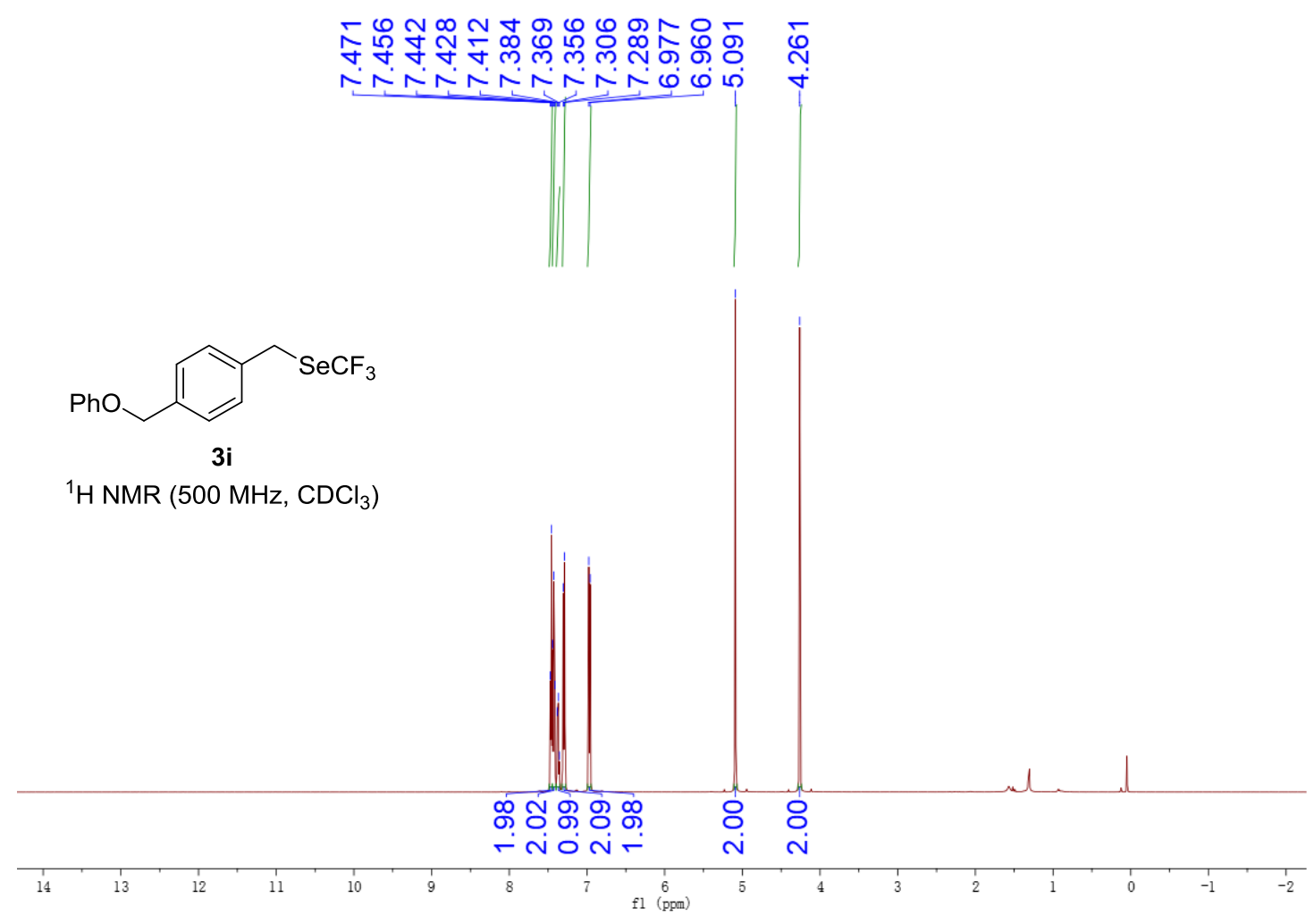

管

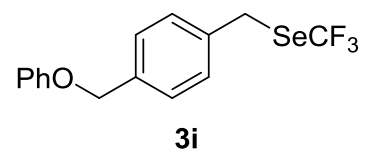

${ }^{19} \mathrm{~F} \mathrm{NMR}\left(471 \mathrm{MHz}, \mathrm{CDCl}_{3}\right)$

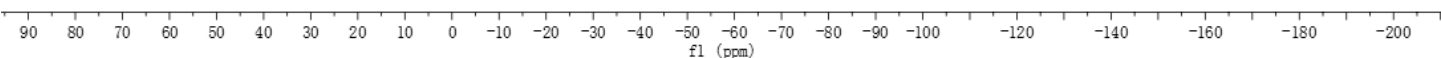



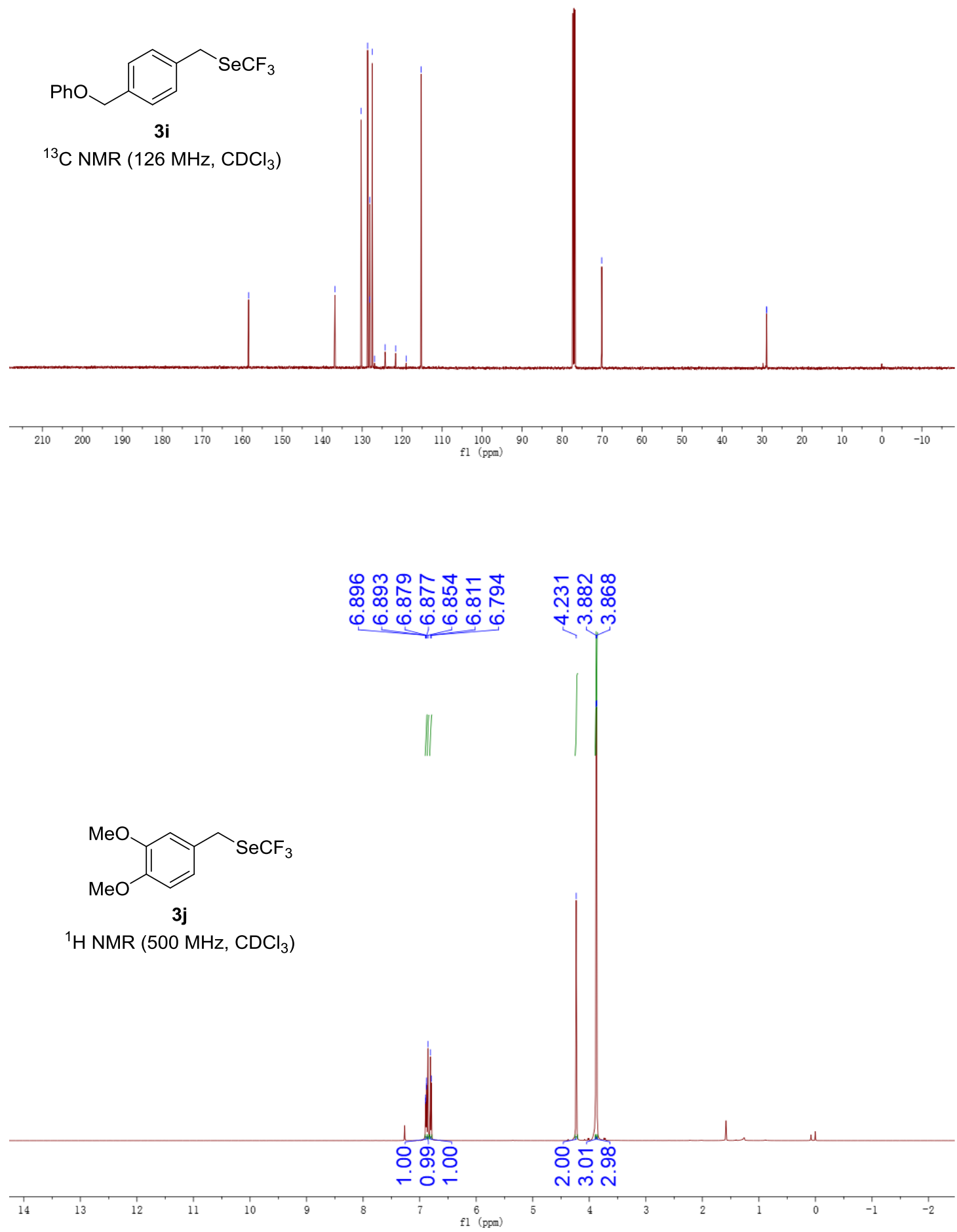


$$
\text { 움 }
$$

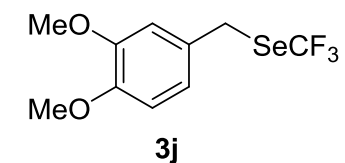

$\left.{ }^{19} \mathrm{~F} \mathrm{NMR} \mathrm{(471} \mathrm{MHz,} \mathrm{CDCl}_{3}\right)$

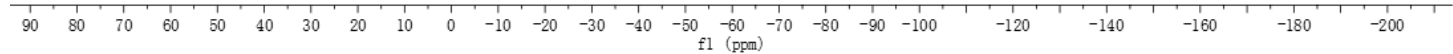

ஸุ

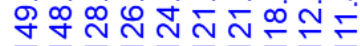

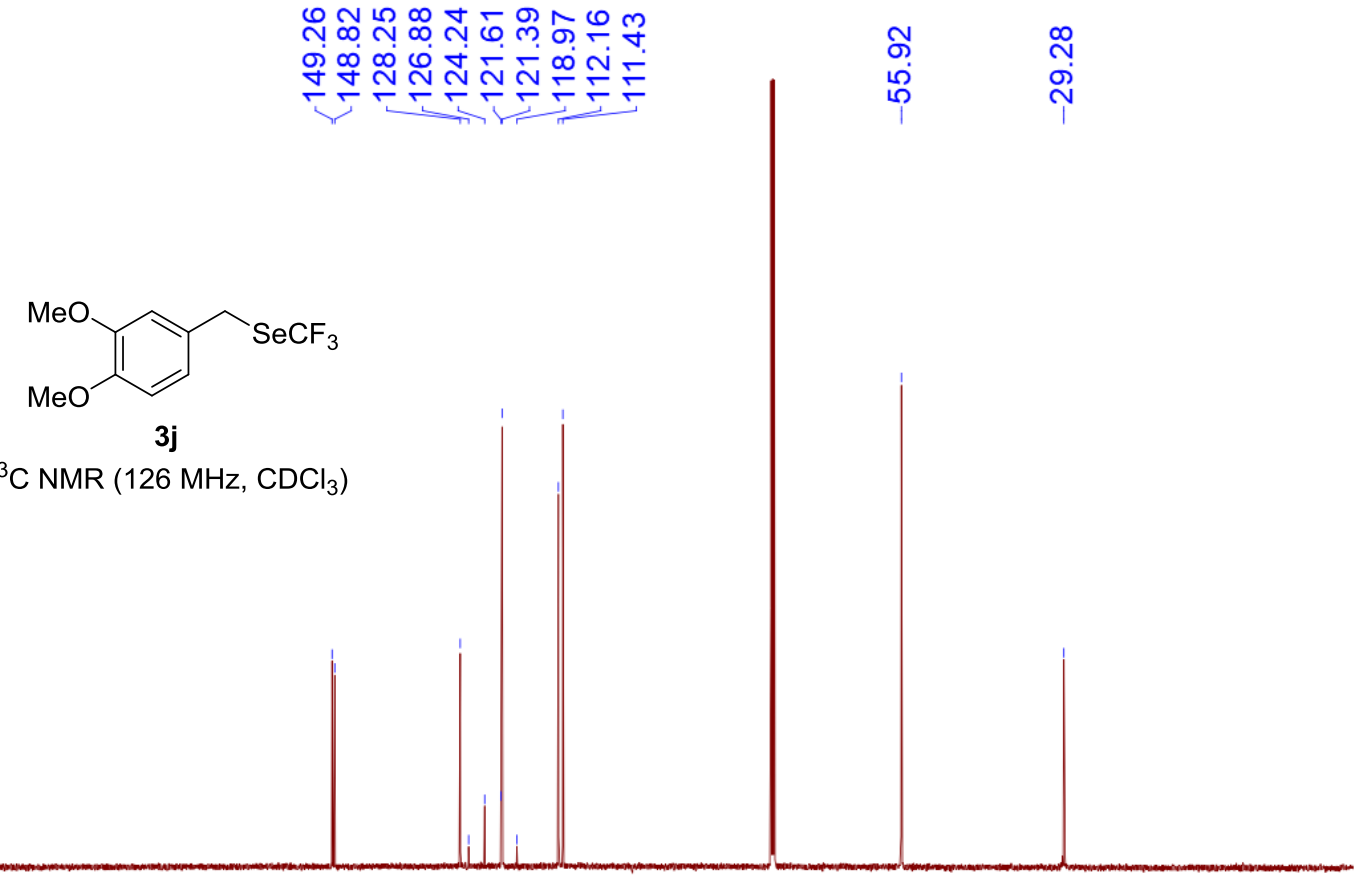

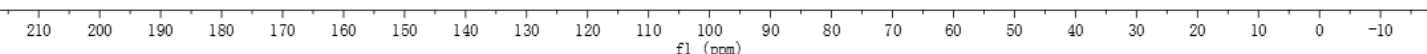




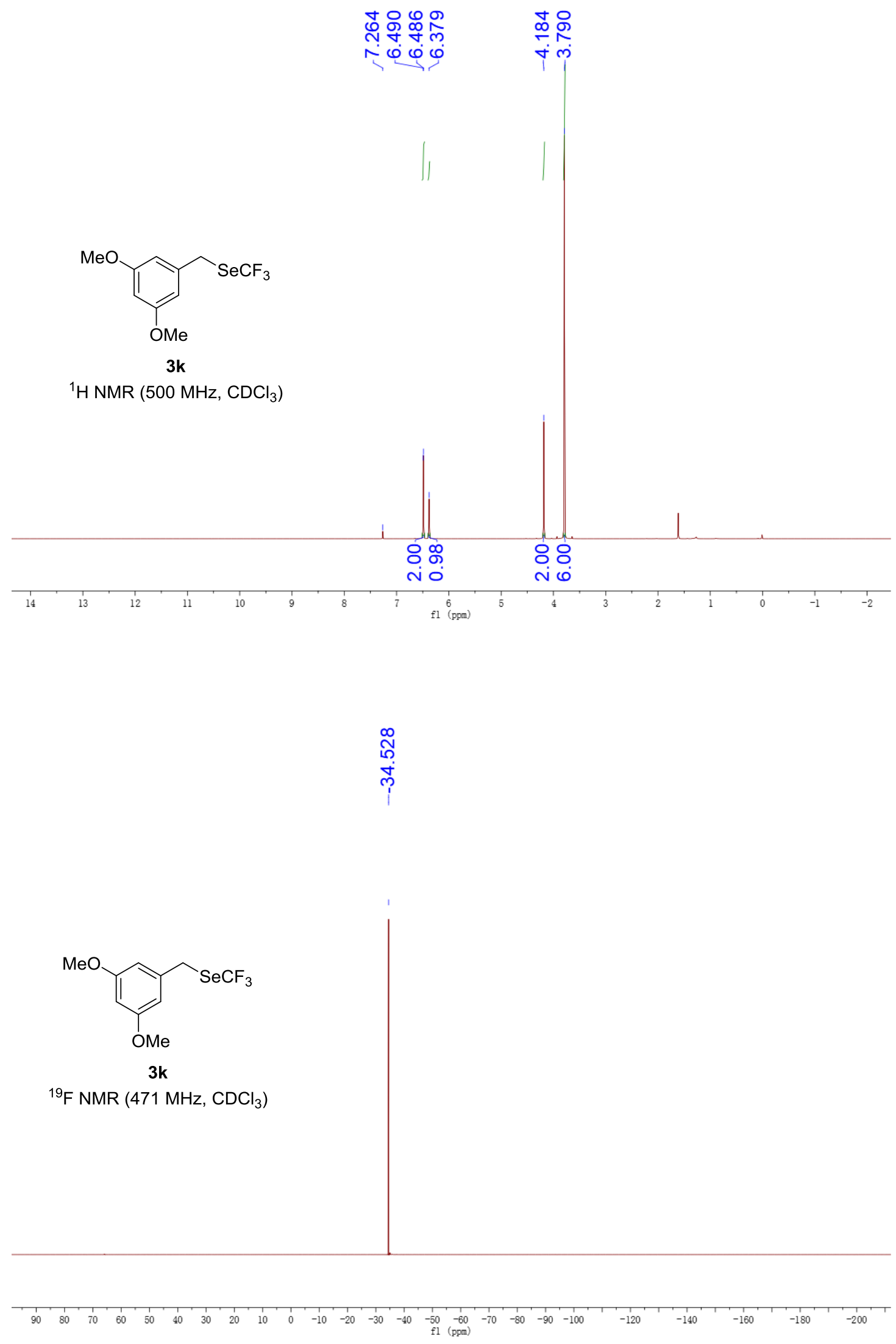



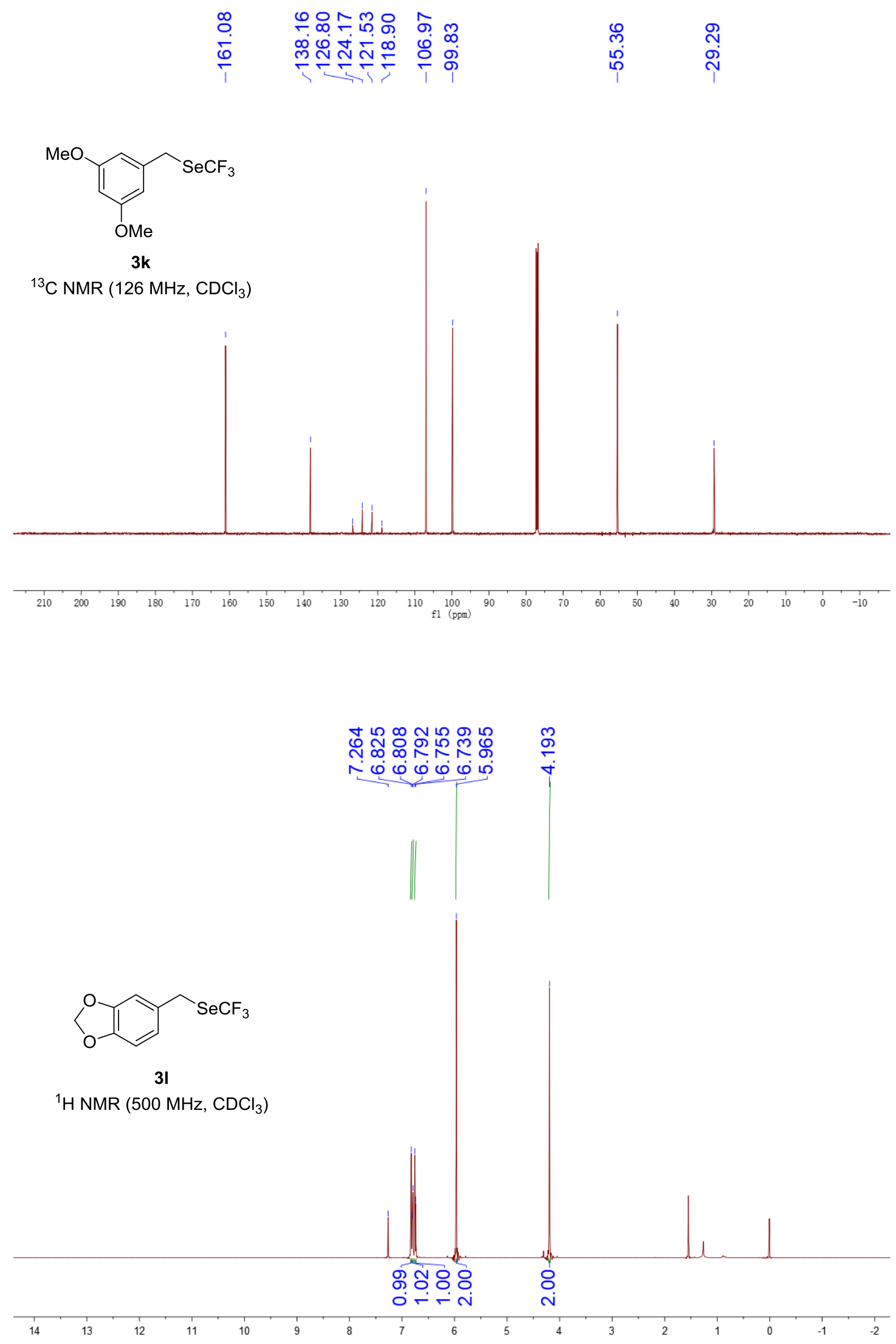


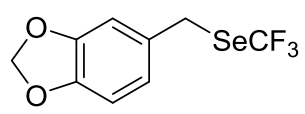

31

${ }^{19} \mathrm{~F} \mathrm{NMR}\left(471 \mathrm{MHz}, \mathrm{CDCl}_{3}\right)$

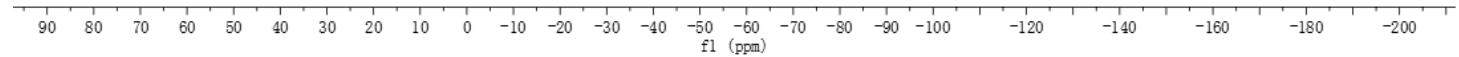

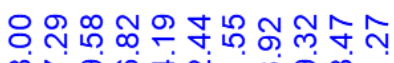

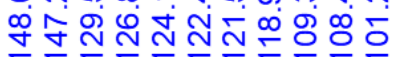

ल이

ฟiं

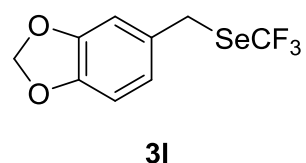

${ }^{13} \mathrm{C}$ NMR (126 MHz, $\mathrm{CDCl}_{3}$ ) 


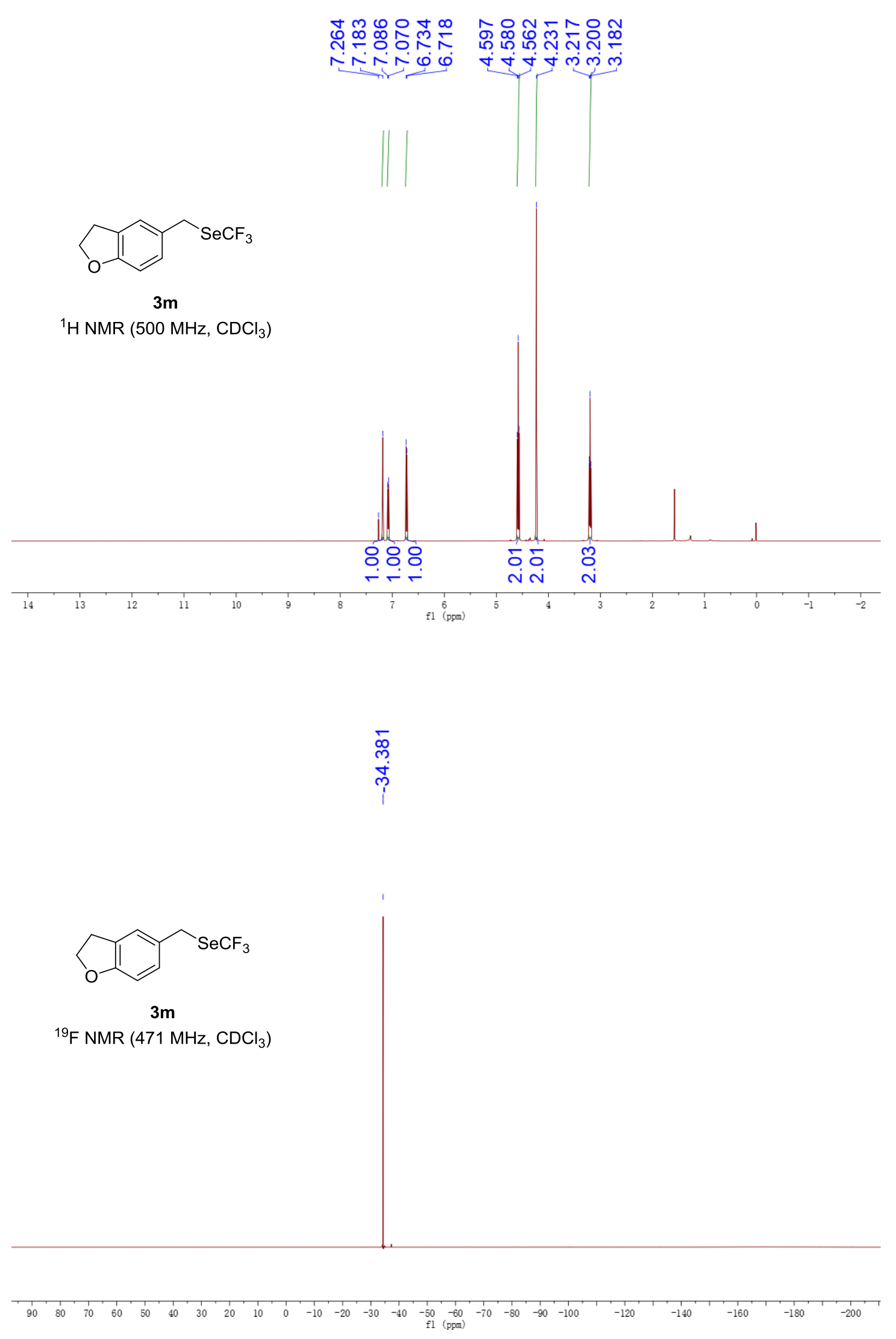



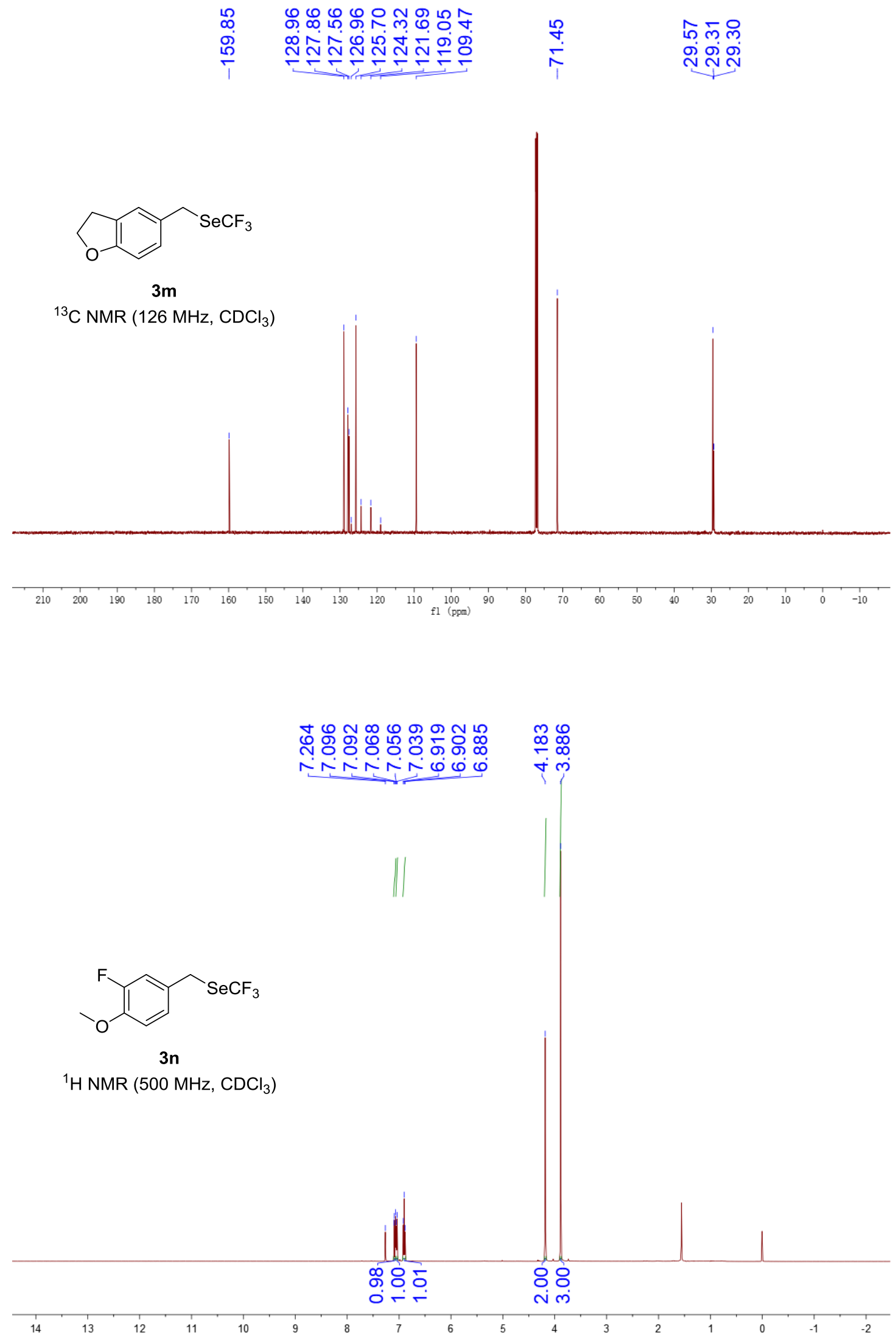

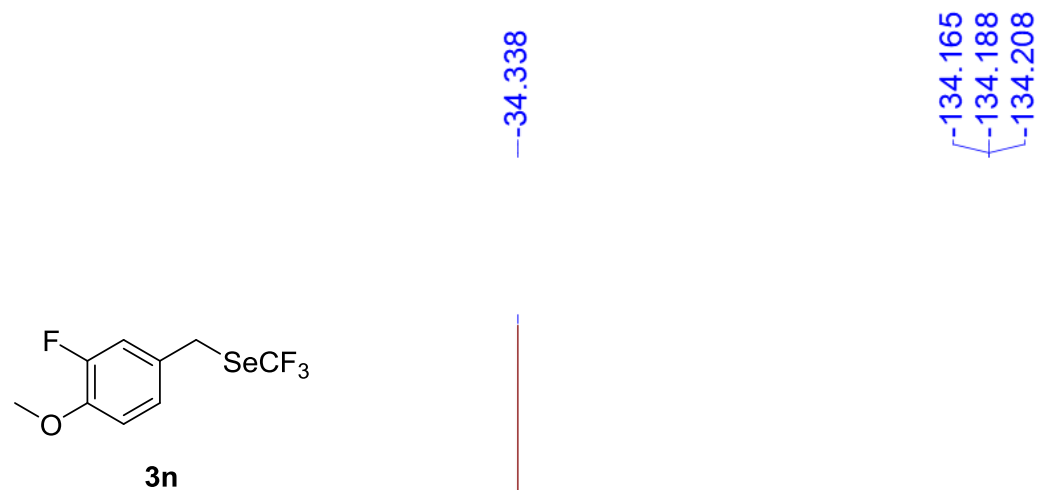

${ }^{19} \mathrm{~F}$ NMR $\left(471 \mathrm{MHz}, \mathrm{CDCl}_{3}\right)$

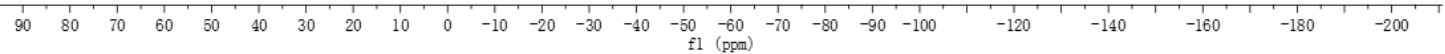

뉸ำำ

กิก

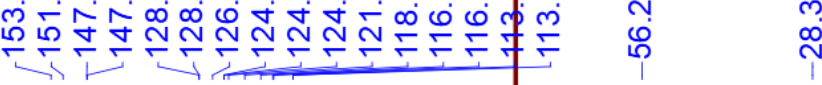

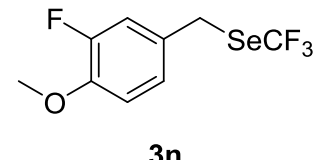

${ }^{13} \mathrm{C} \mathrm{NMR}\left(126 \mathrm{MHz}, \mathrm{CDCl}_{3}\right)$

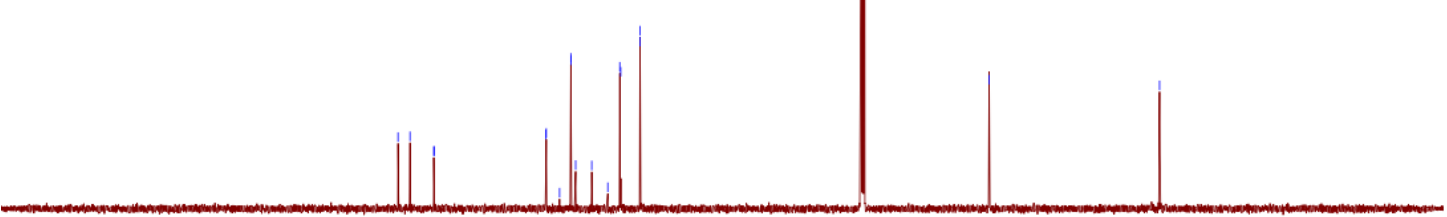

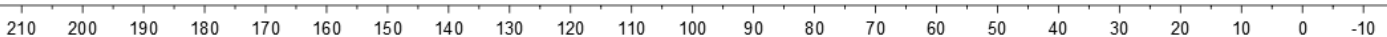




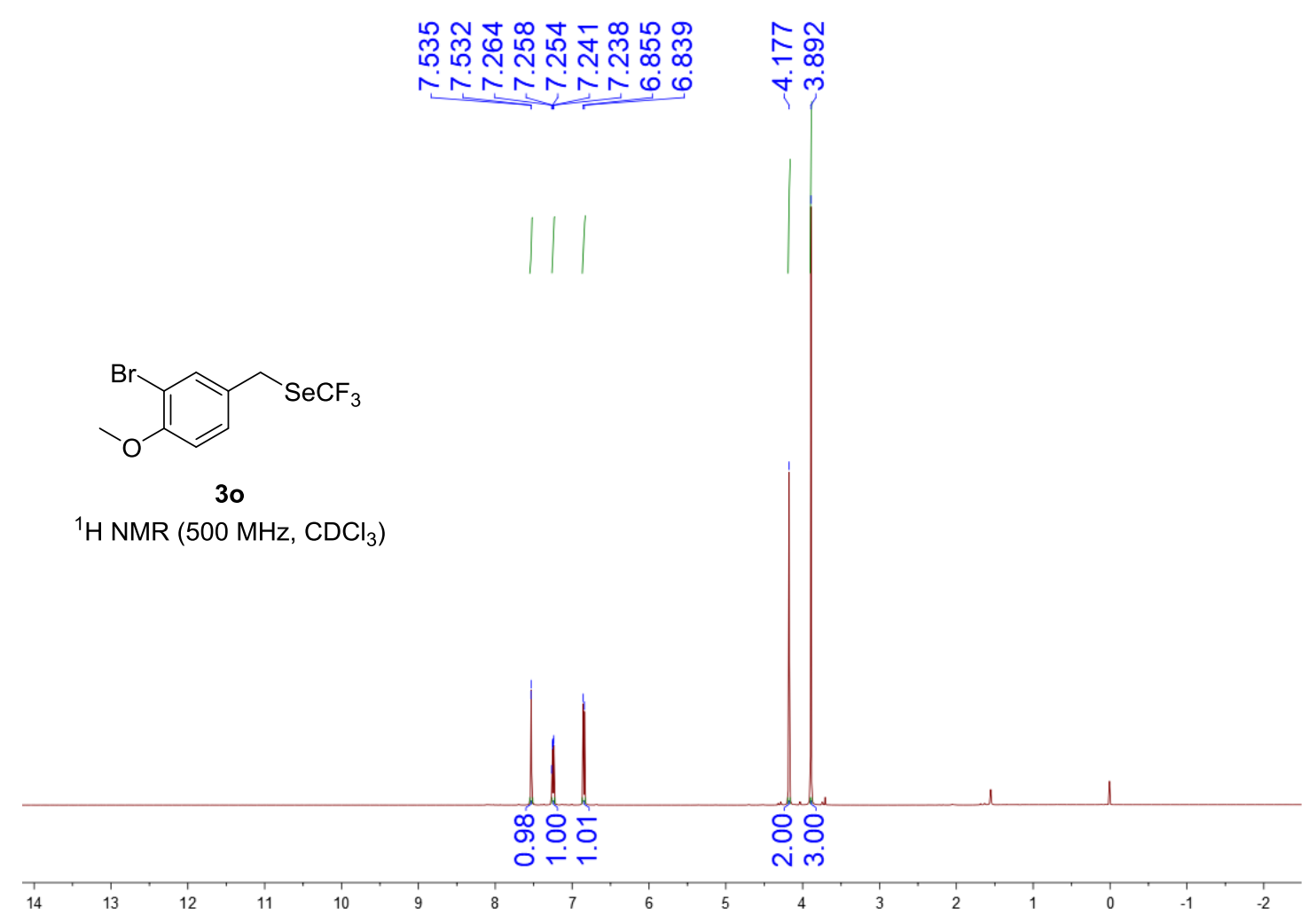

\section{m}

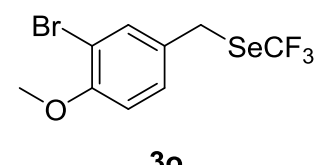

${ }^{19} \mathrm{~F}$ NMR (471 $\mathrm{MHz}, \mathrm{CDCl}_{3}$ )

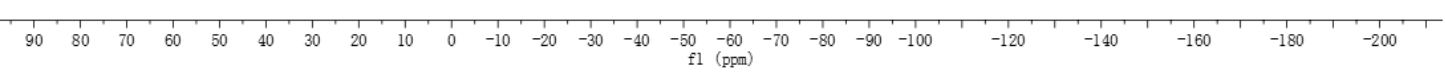



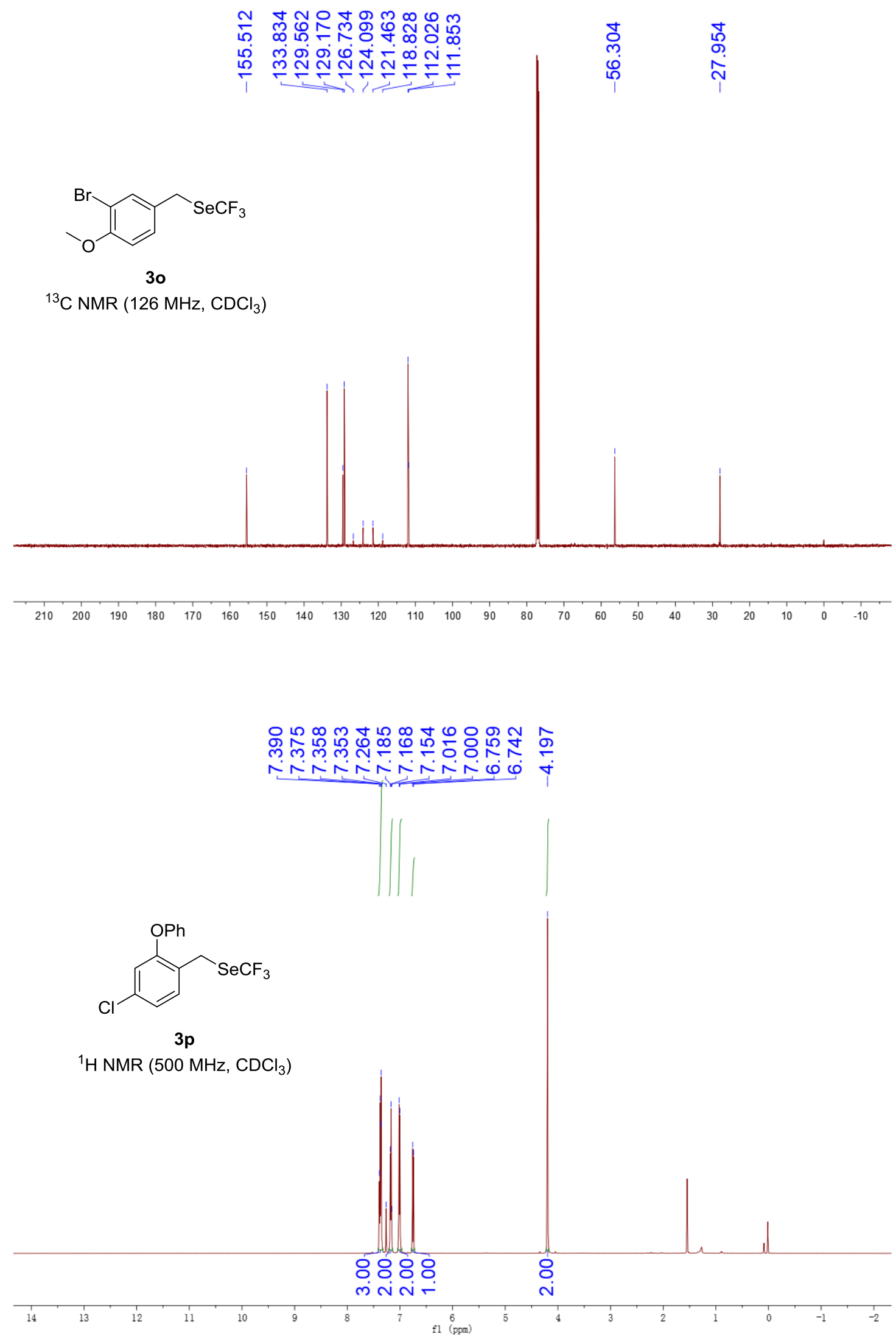


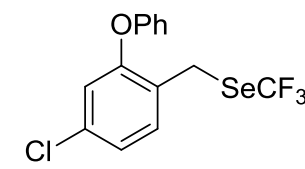

$3 p$

${ }^{19} \mathrm{~F} \mathrm{NMR}\left(471 \mathrm{MHz}, \mathrm{CDCl}_{3}\right)$

ก்ุ

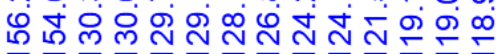

8.

ํำ

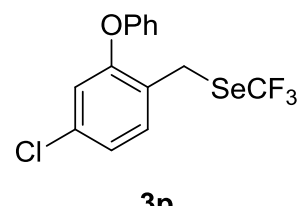

3p

${ }^{13} \mathrm{C}$ NMR $\left(476 \mathrm{MHz}, \mathrm{CDCl}_{3}\right)$

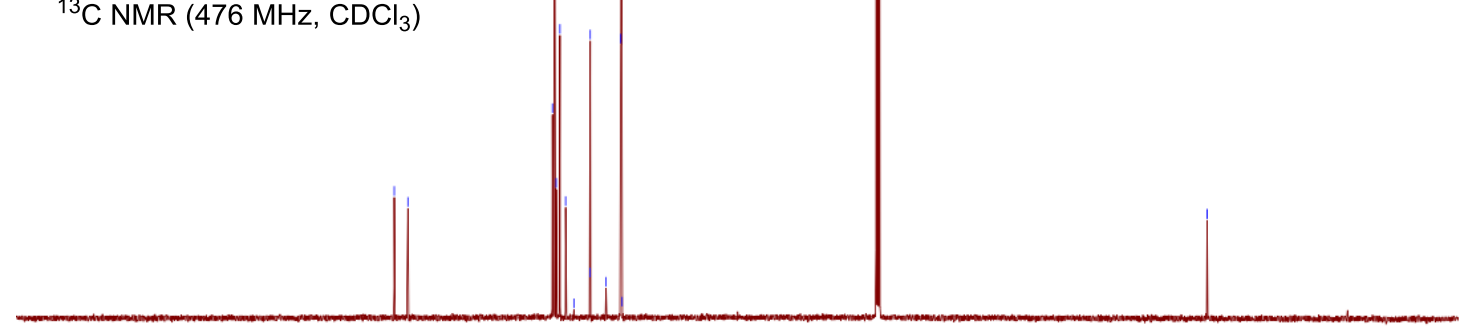

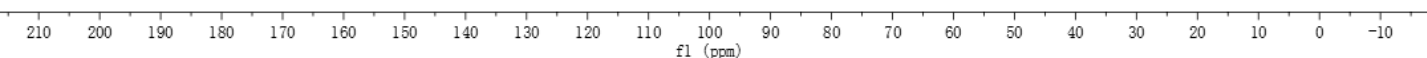




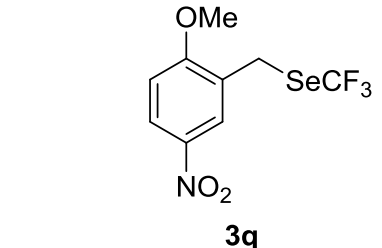

$3 q$

${ }^{1} \mathrm{H}$ NMR $\left(500 \mathrm{MHz}, \mathrm{CDCl}_{3}\right)$
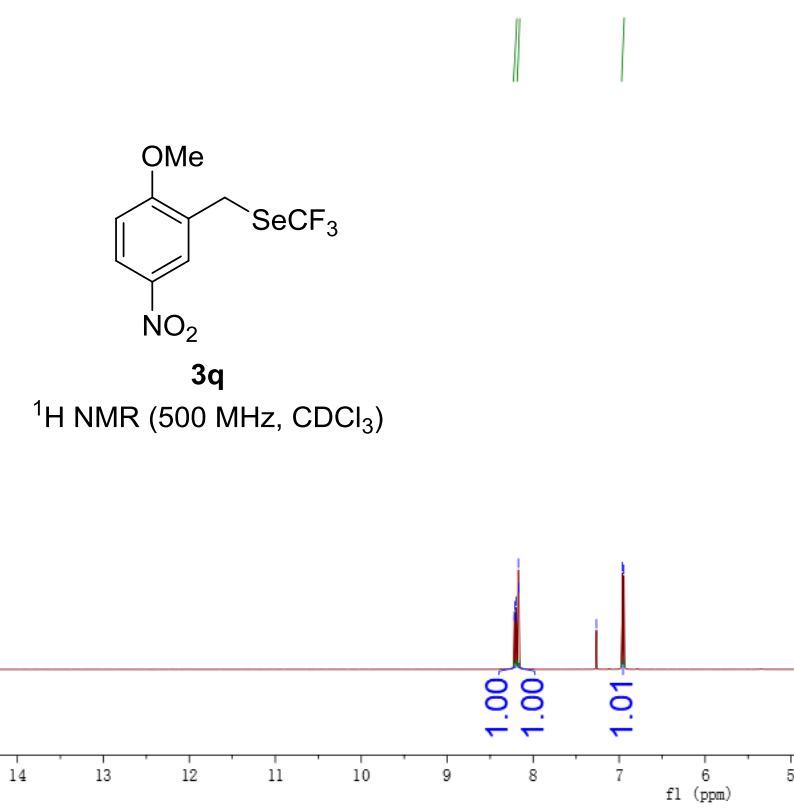

o 8

กั

กิ

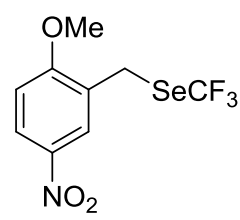

$3 q$

${ }^{19} \mathrm{~F}$ NMR $\left(471 \mathrm{MHz}, \mathrm{CDCl}_{3}\right)$

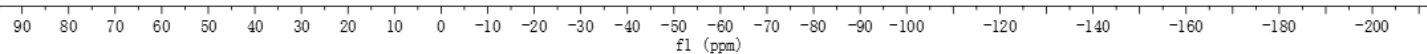




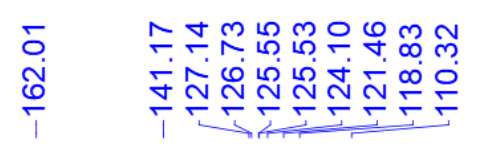
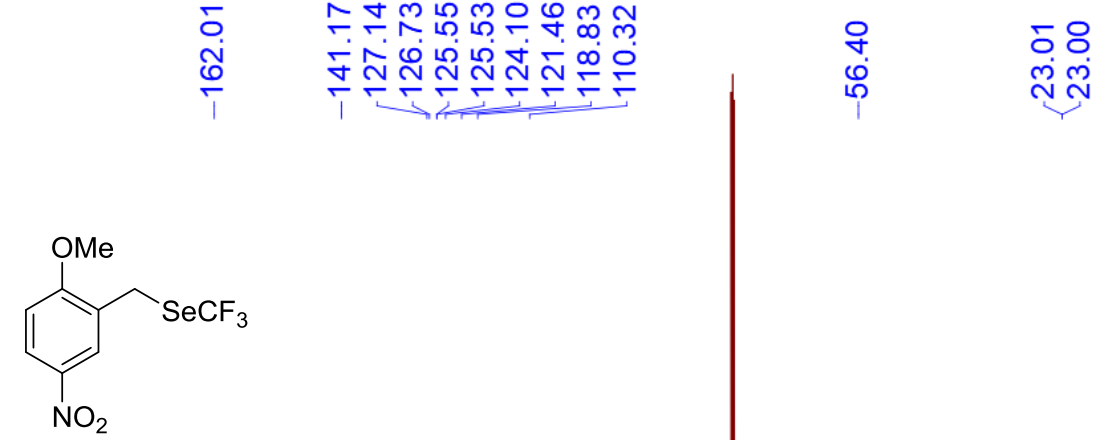

$3 q$

${ }^{13} \mathrm{C}$ NMR $\left(126 \mathrm{MHz}, \mathrm{CDCl}_{3}\right)$
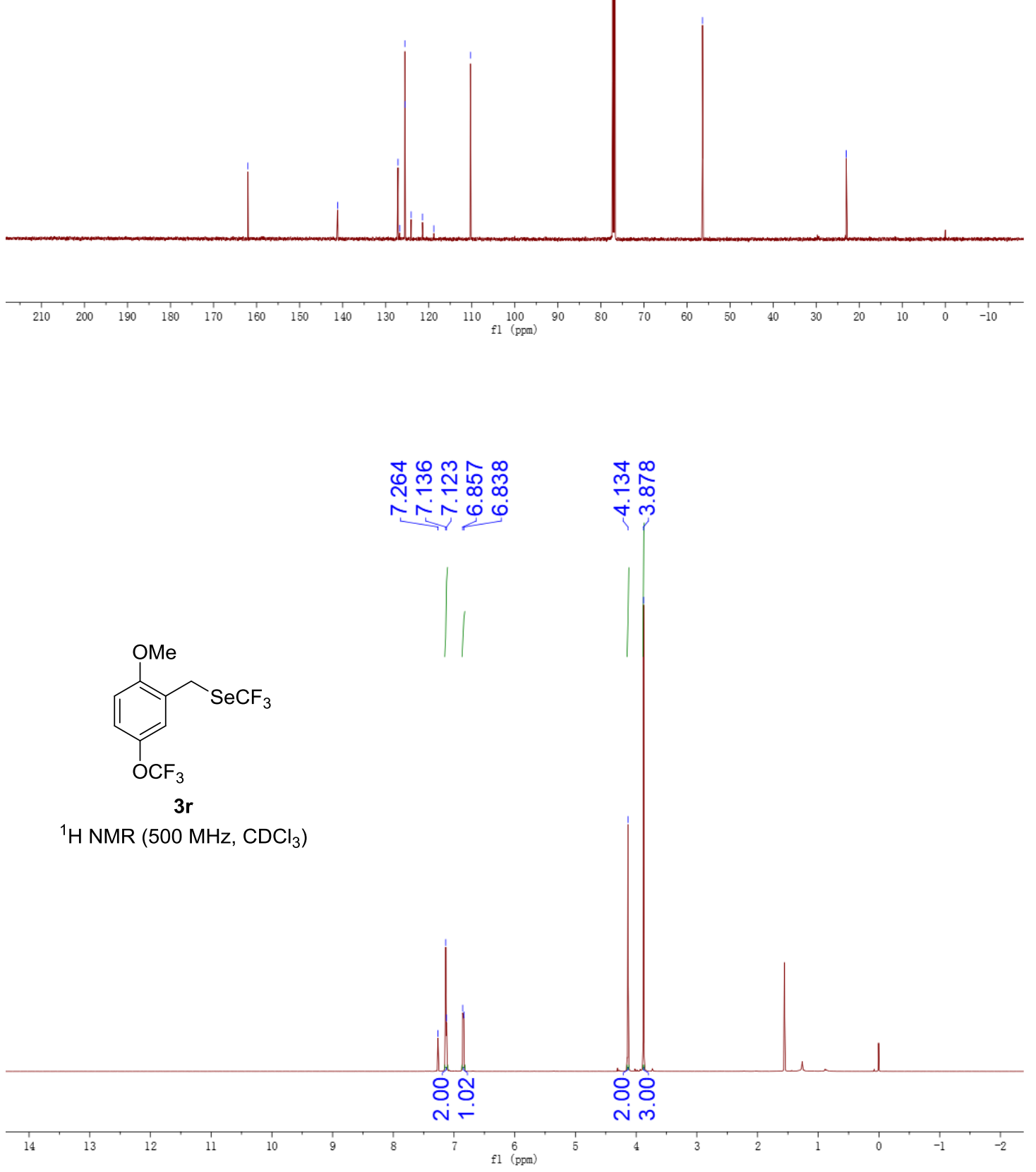

S64 


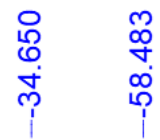

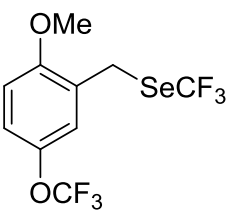

$3 r$

${ }^{19} \mathrm{~F} \mathrm{NMR}\left(471 \mathrm{MHz}, \mathrm{CDCl}_{3}\right)$
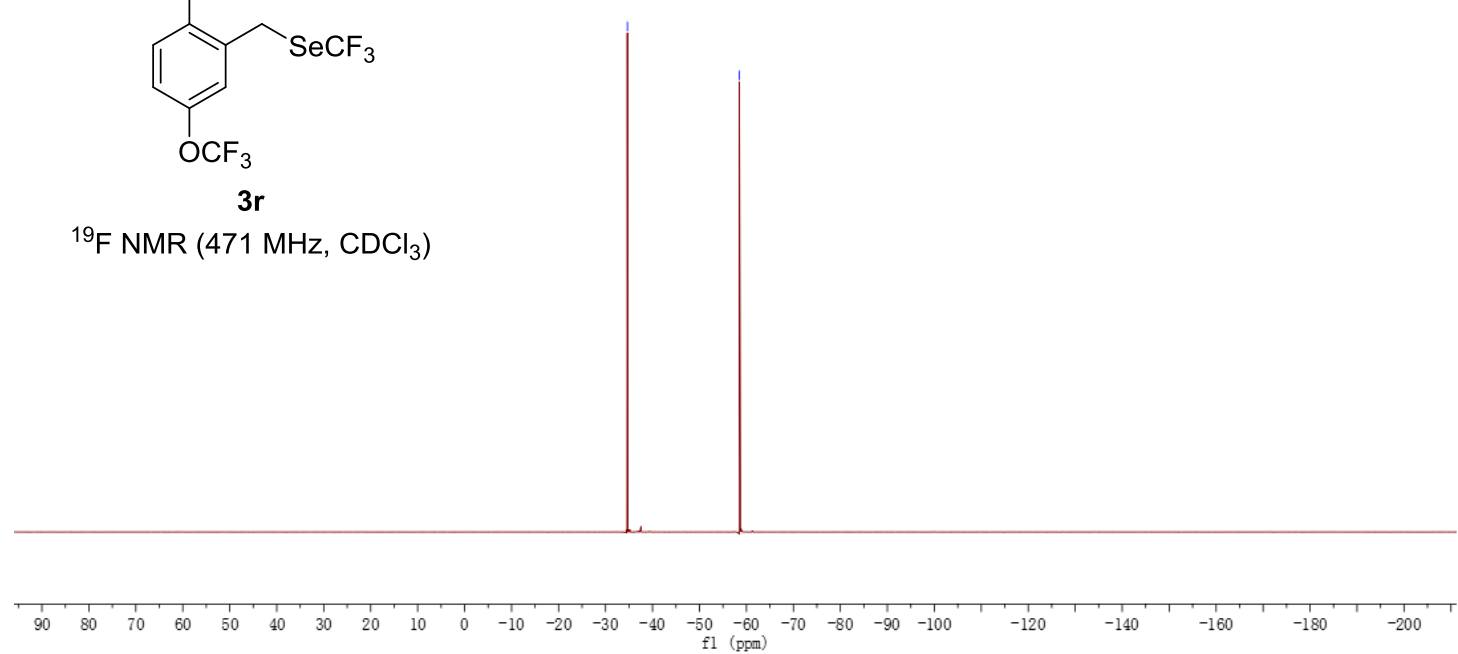

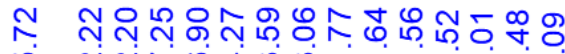

L

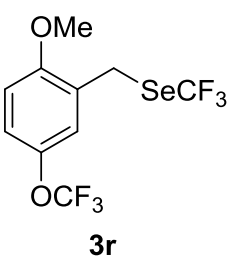

${ }^{13} \mathrm{C} \mathrm{NMR}\left(126 \mathrm{MHz}, \mathrm{CDCl}_{3}\right)$
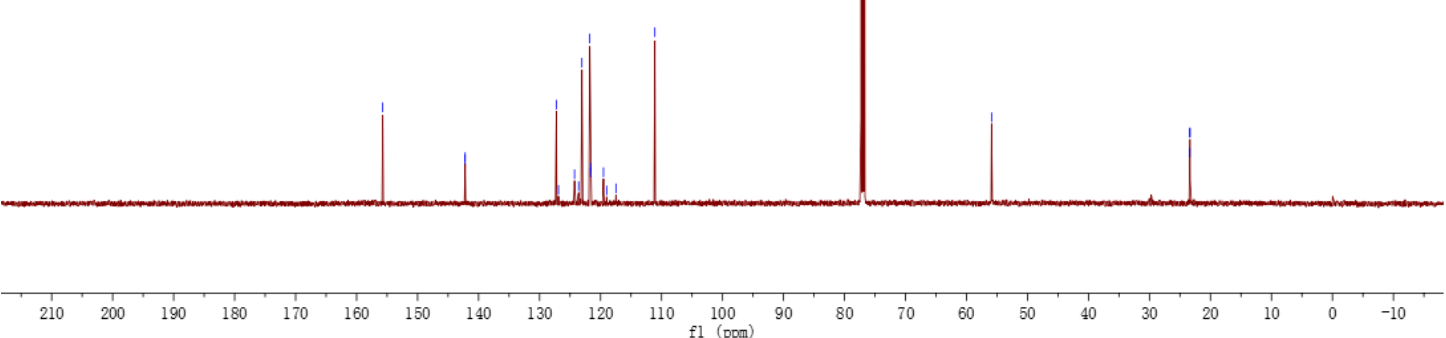


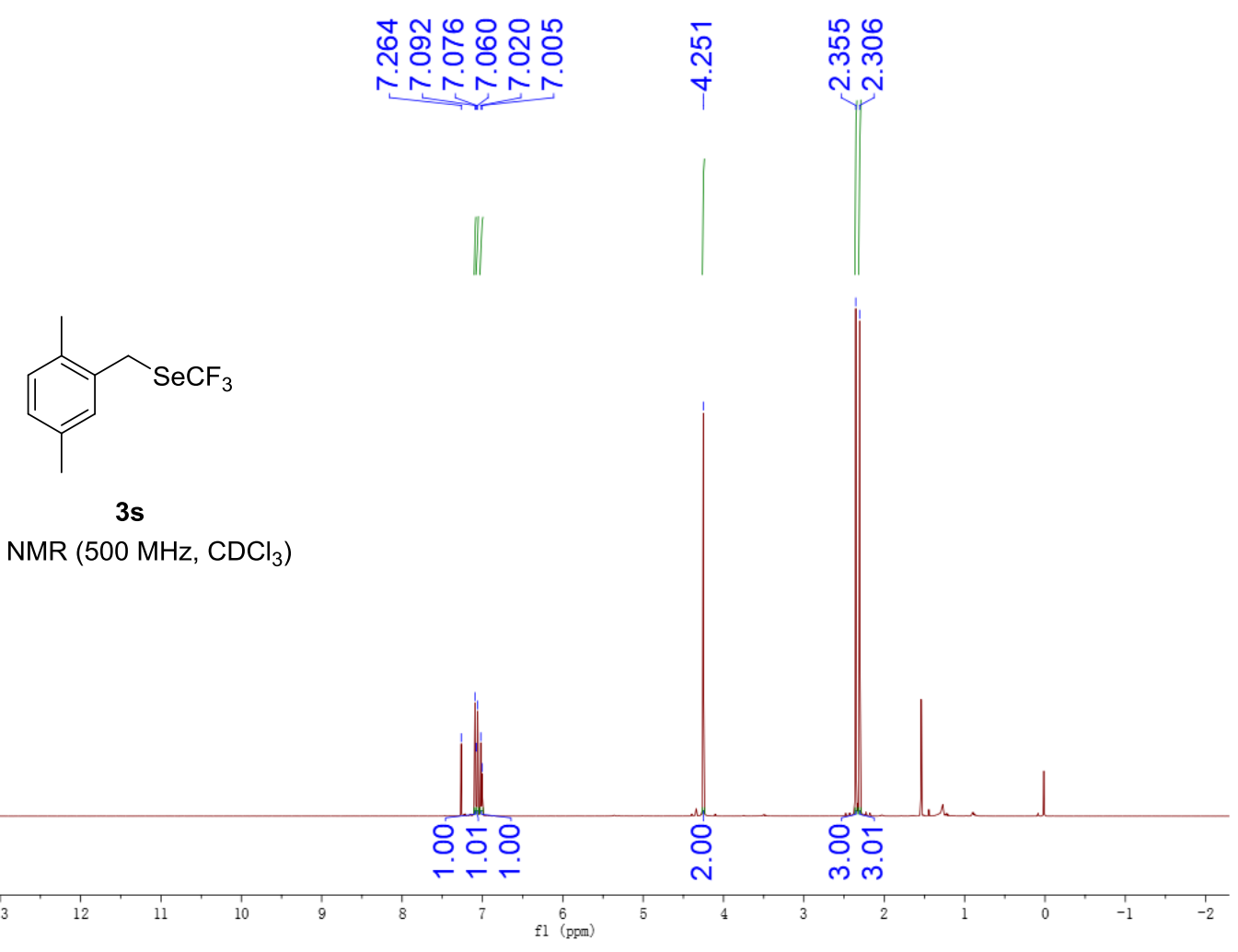

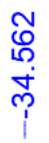

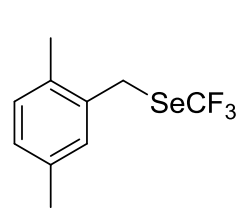

3s

${ }^{19} \mathrm{~F}$ NMR $\left(471 \mathrm{MHz}, \mathrm{CDCl}_{3}\right.$ )

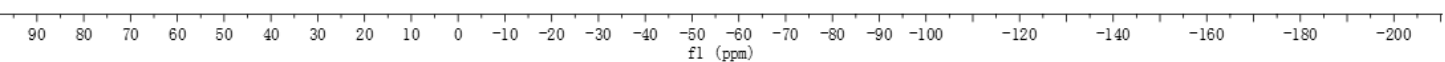



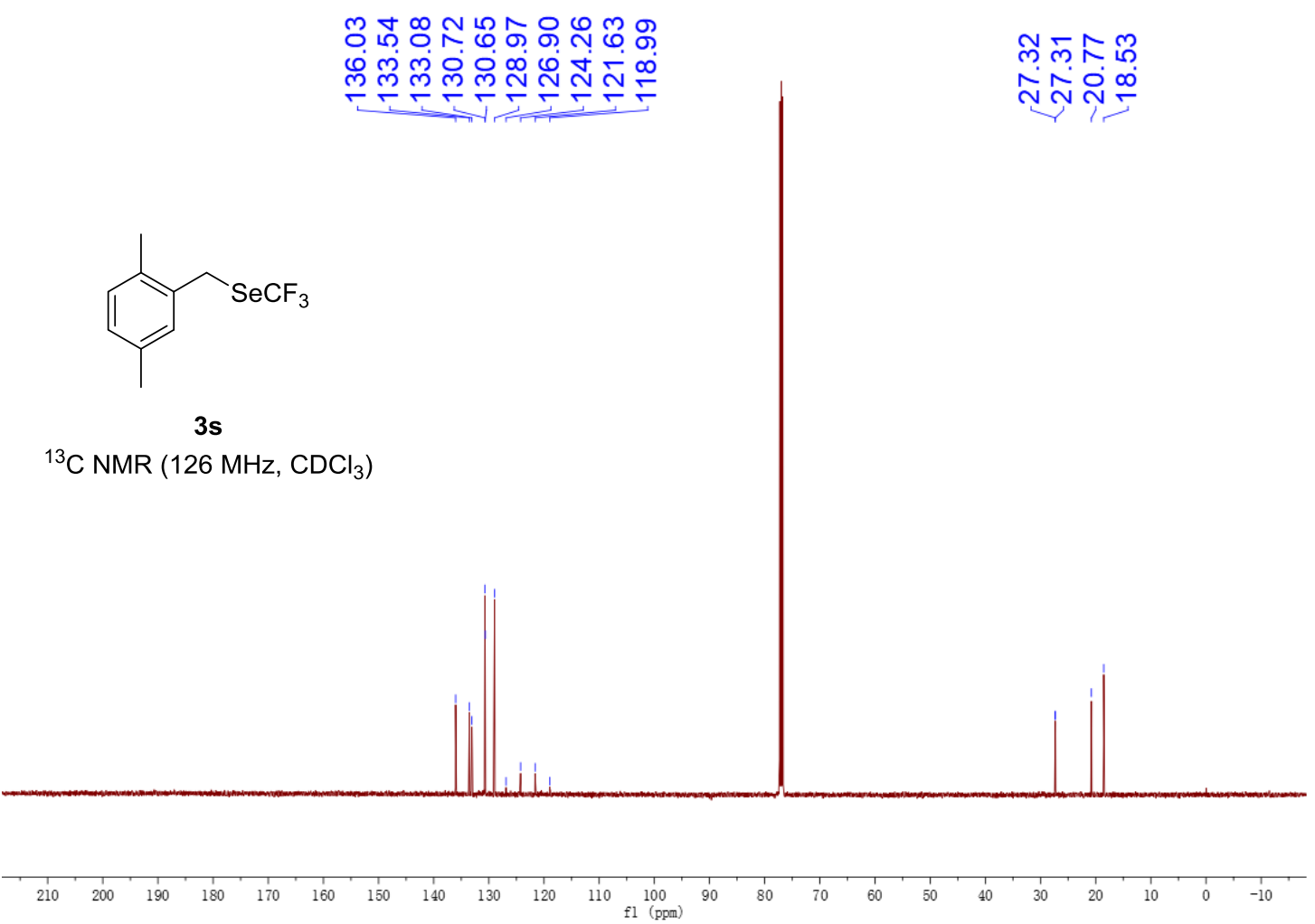

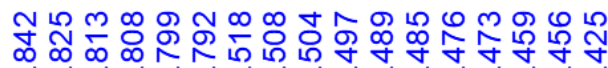

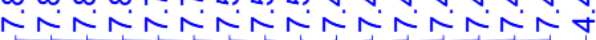

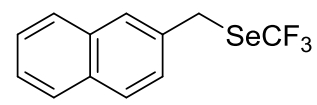

$3 \mathbf{t}$

${ }^{1} \mathrm{H}$ NMR $\left(500 \mathrm{MHz}, \mathrm{CDCl}_{3}\right)$

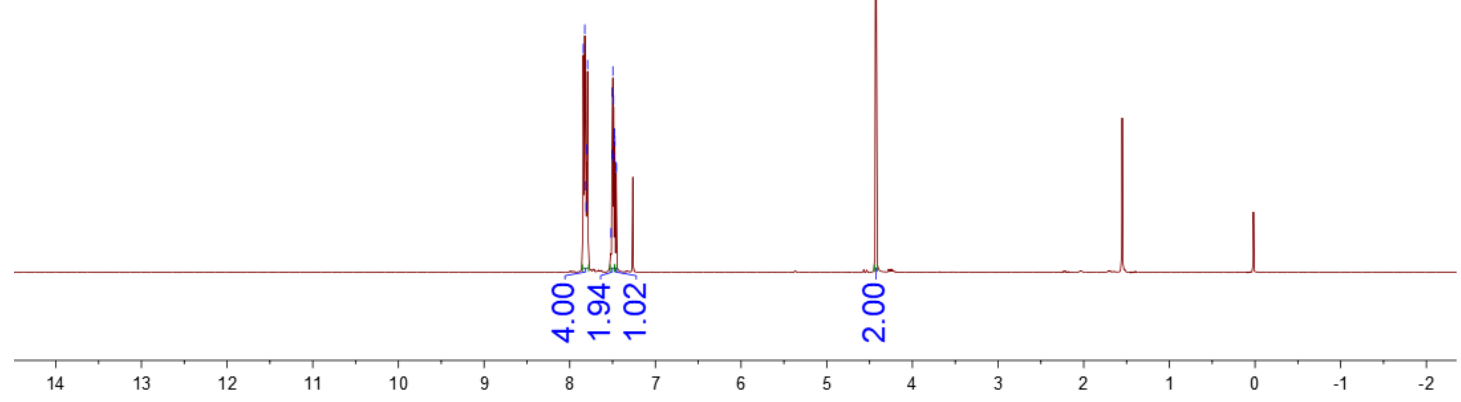


<smiles>F[C-](F)[Ge]Cc1ccc2ccccc2c1</smiles>

$3 t$

${ }^{19} \mathrm{~F} \mathrm{NMR}\left(471 \mathrm{MHz}, \mathrm{CDCl}_{3}\right)$

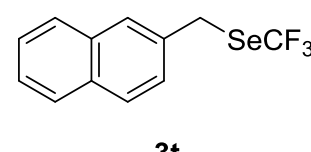

3t

${ }^{13} \mathrm{C} \mathrm{NMR}\left(126 \mathrm{MHz}, \mathrm{CDCl}_{3}\right)$

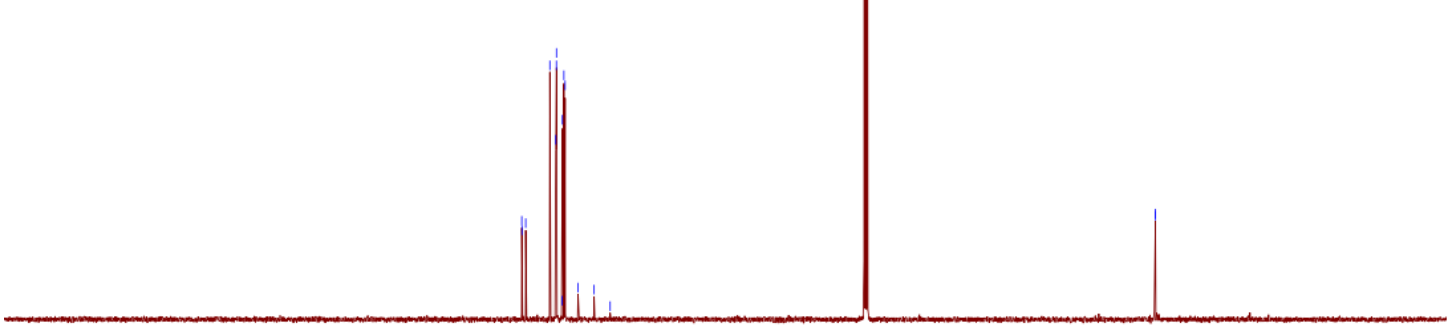

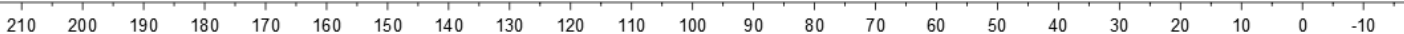




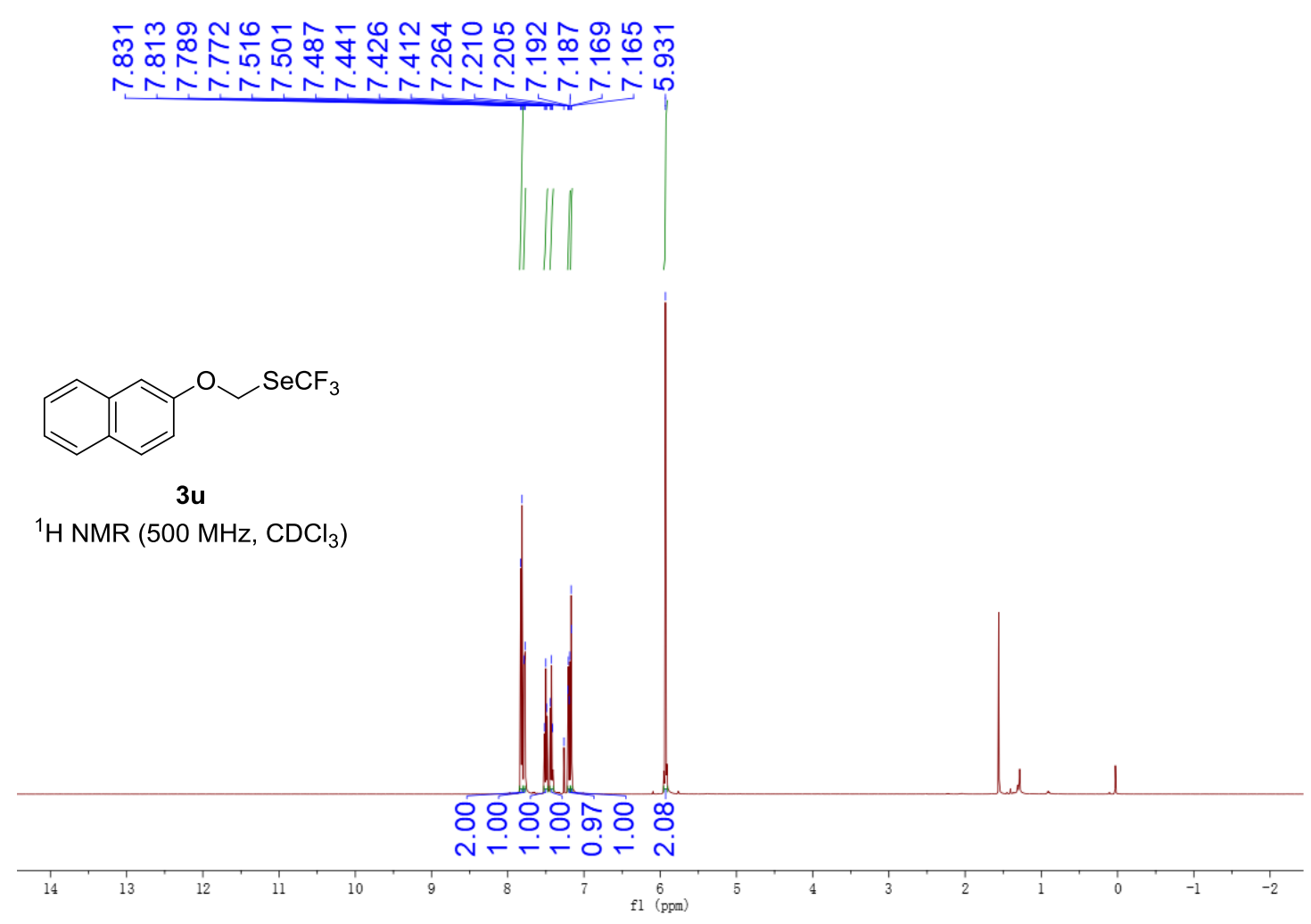

ì্

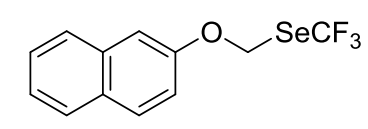

$3 \mathrm{u}$

${ }^{19} \mathrm{~F} \mathrm{NMR}\left(471 \mathrm{MHz}, \mathrm{CDCl}_{3}\right)$ 


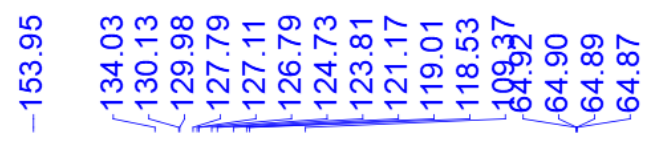
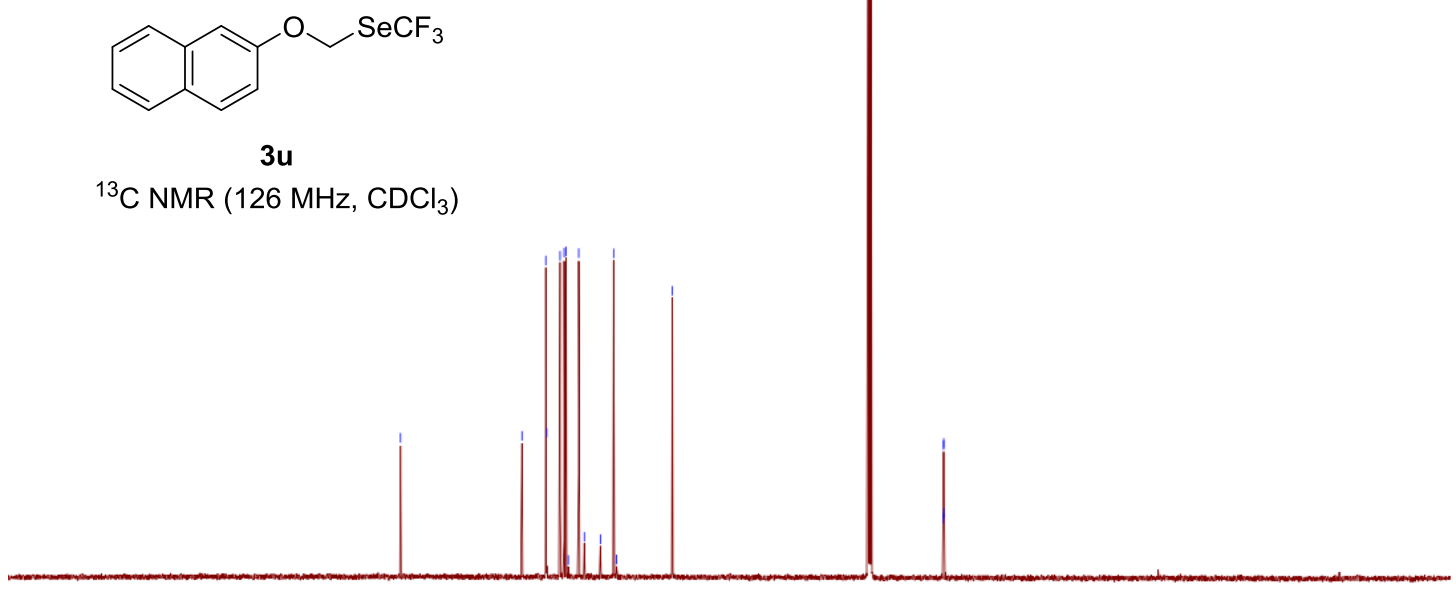

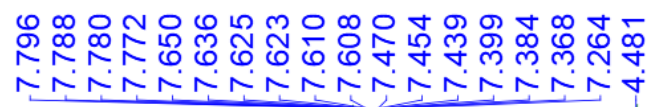
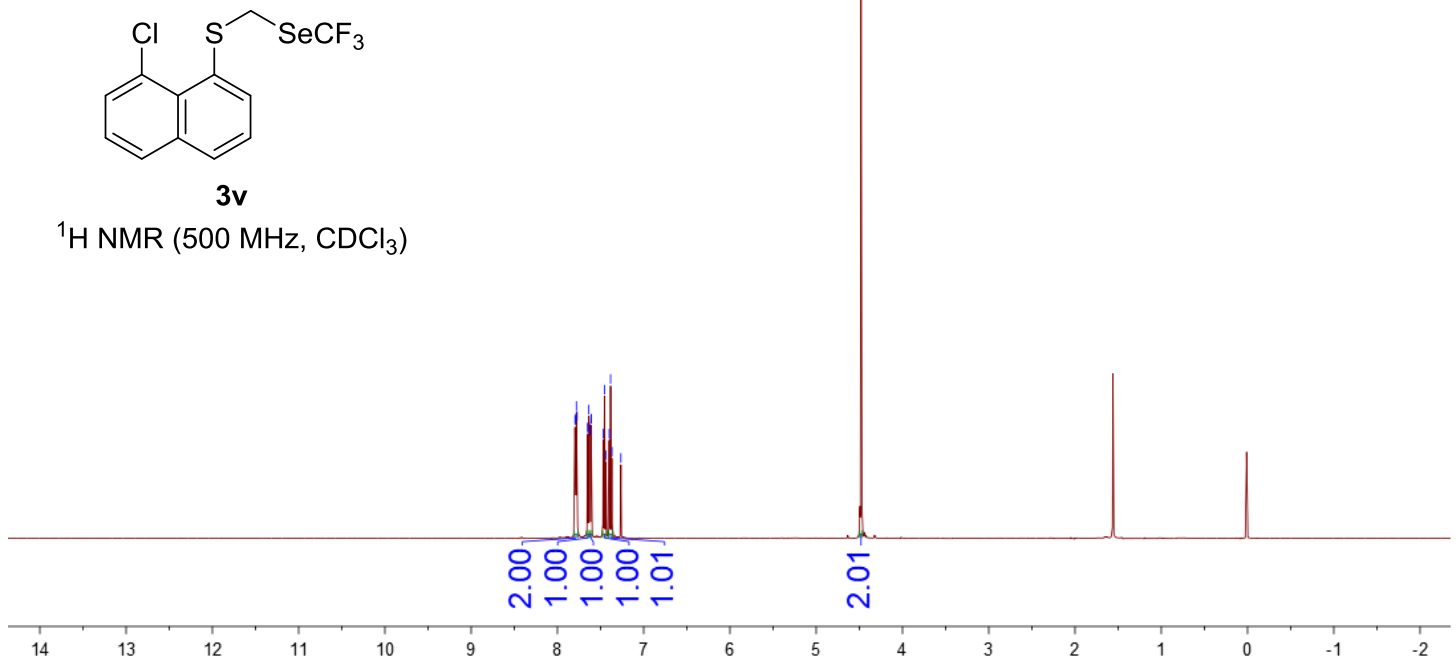

S70 


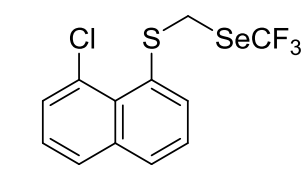

$3 \mathbf{v}$

${ }^{19} \mathrm{~F} \mathrm{NMR}\left(471 \mathrm{MHz}, \mathrm{CDCl}_{3}\right)$

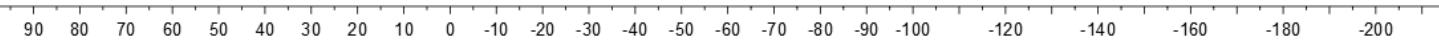

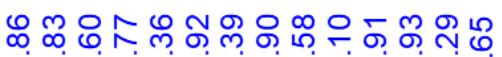

फัต

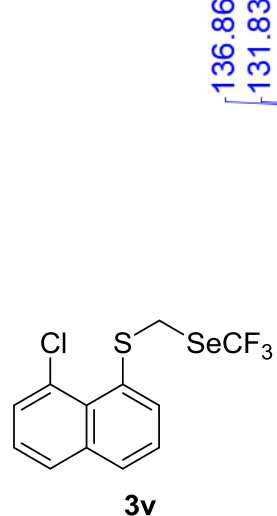

요

ஓं

${ }^{13} \mathrm{C}$ NMR (126 MHz, $\left.\mathrm{CDCl}_{3}\right)$

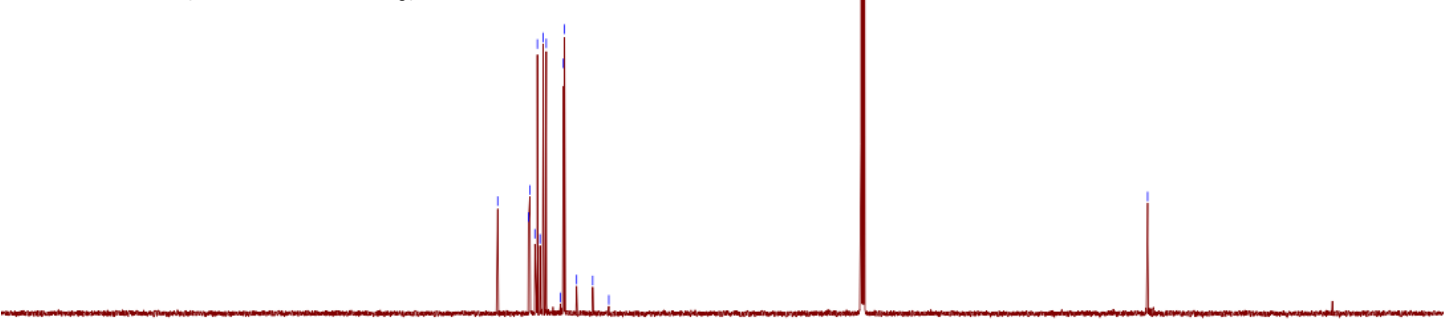

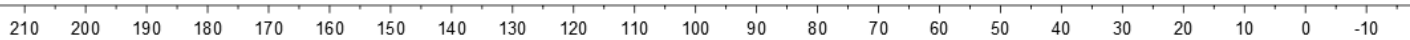




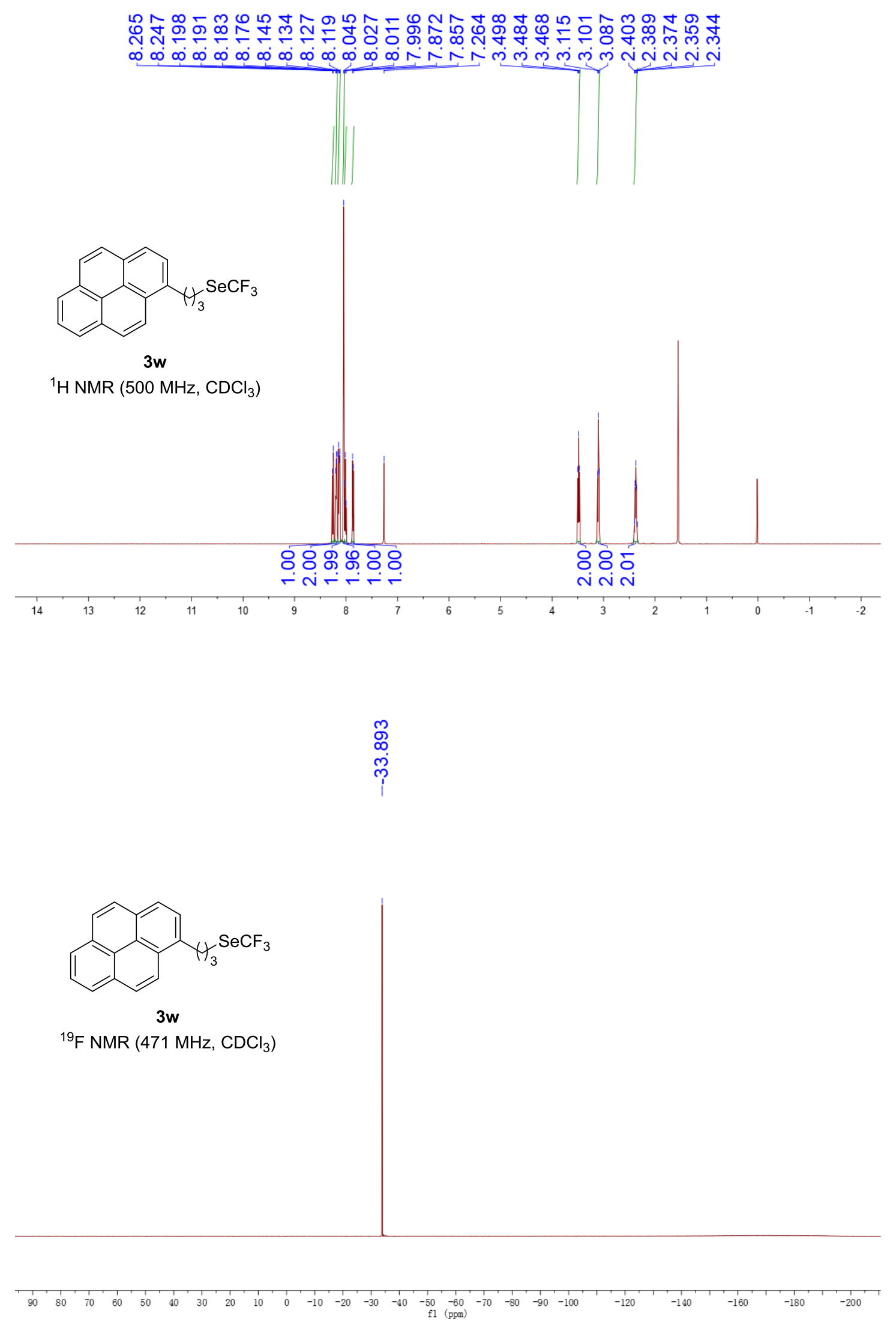




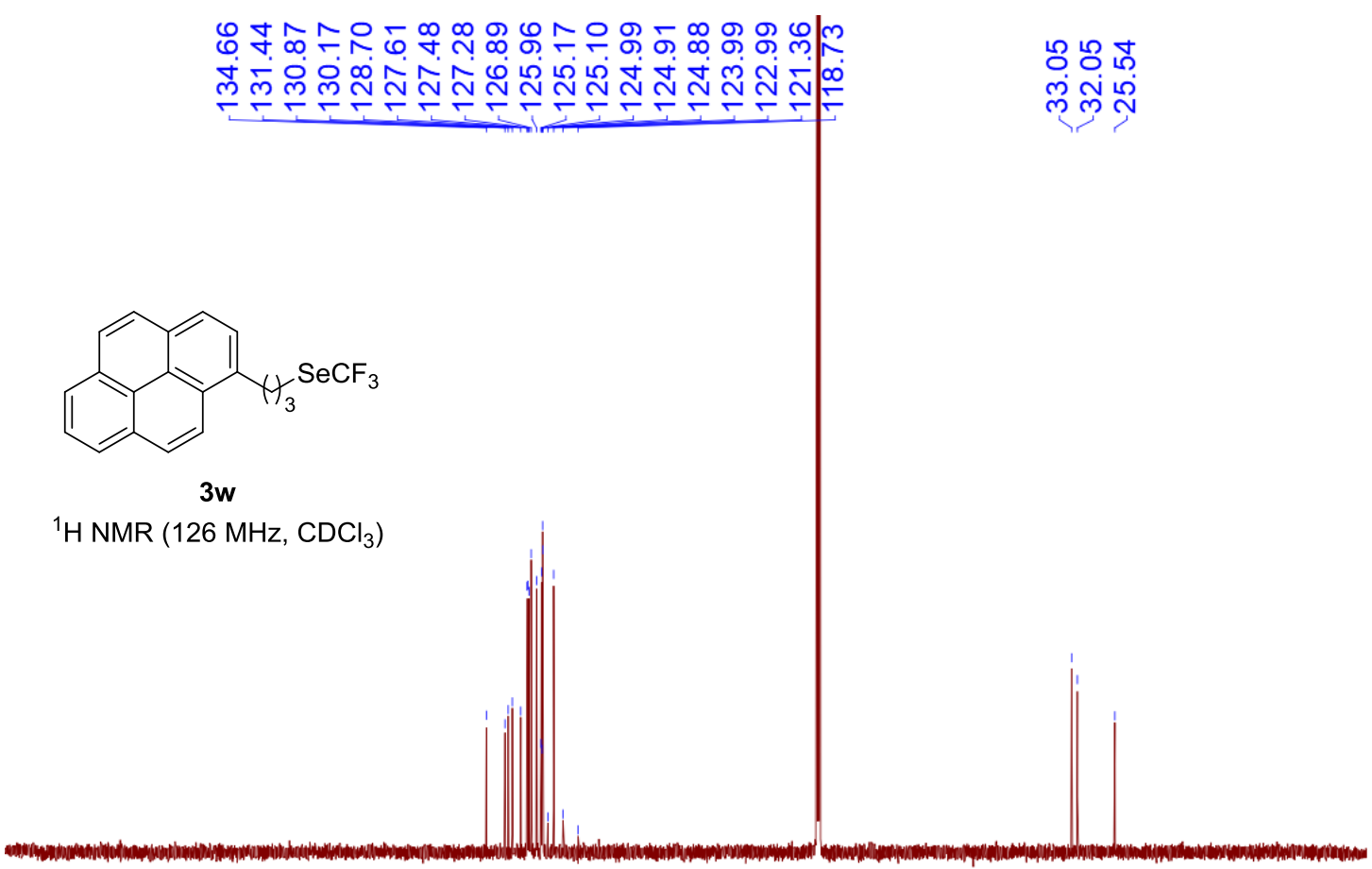

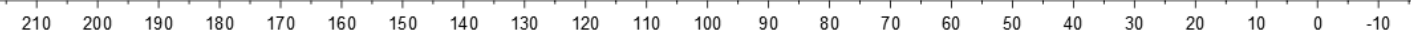

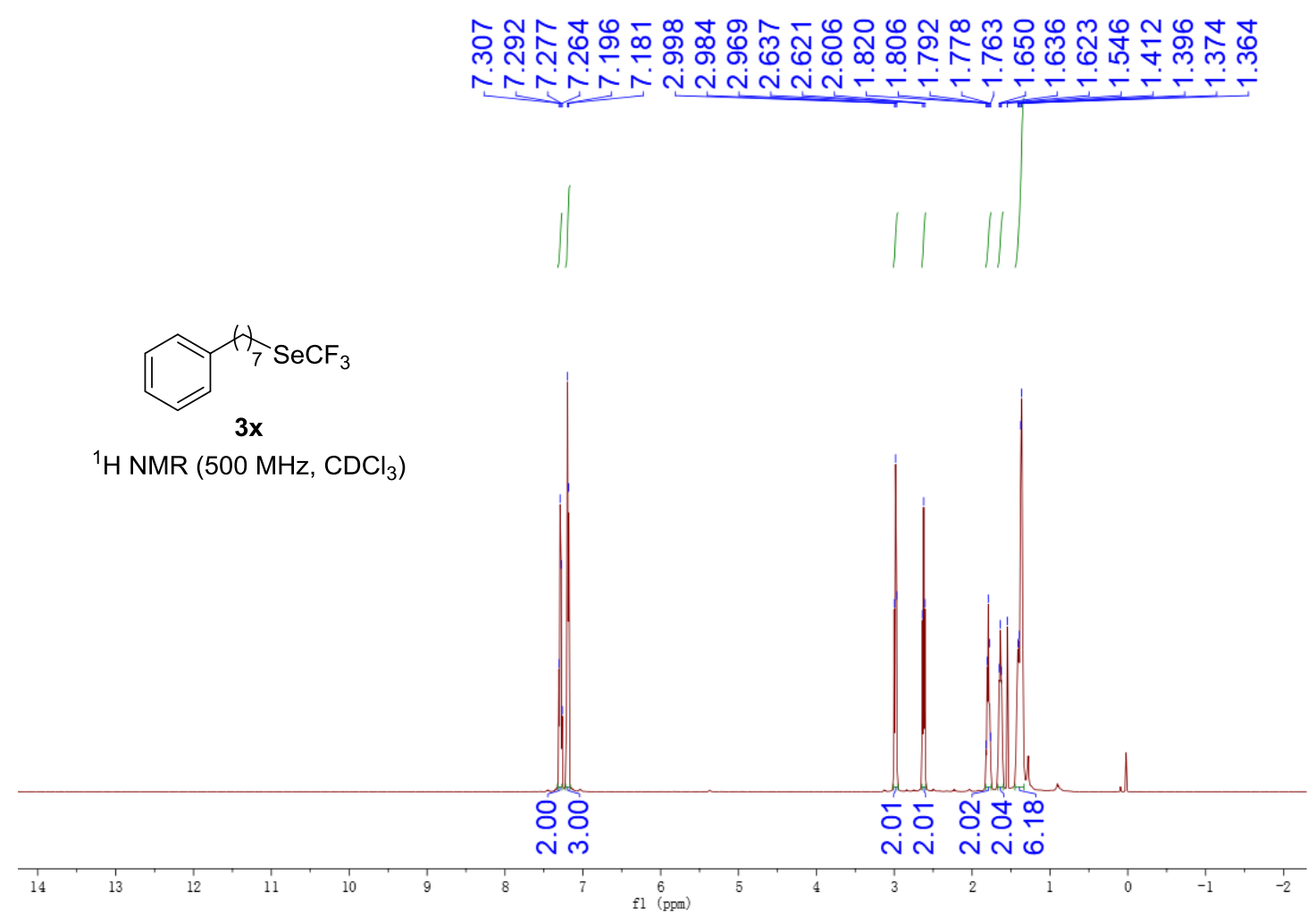




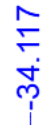

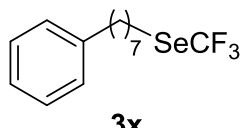

${ }^{19} \mathrm{~F} \mathrm{NMR}\left(471 \mathrm{MHz}, \mathrm{CDCl}_{3}\right)$

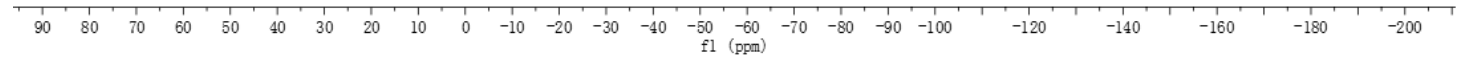

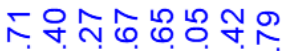

ษั ํํำ

б

மூ

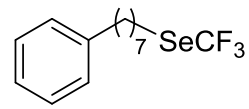

$3 \mathbf{x}$

${ }^{13} \mathrm{C} \mathrm{NMR}\left(126 \mathrm{MHz}, \mathrm{CDCl}_{3}\right)$

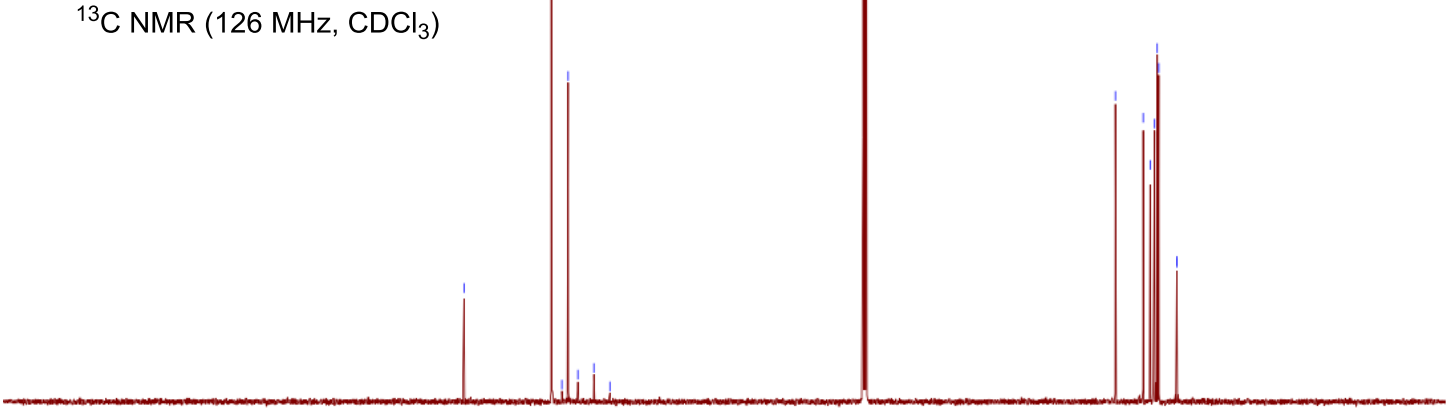

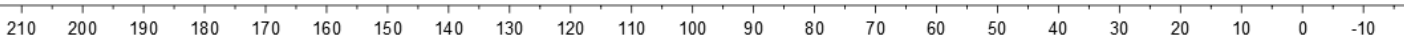




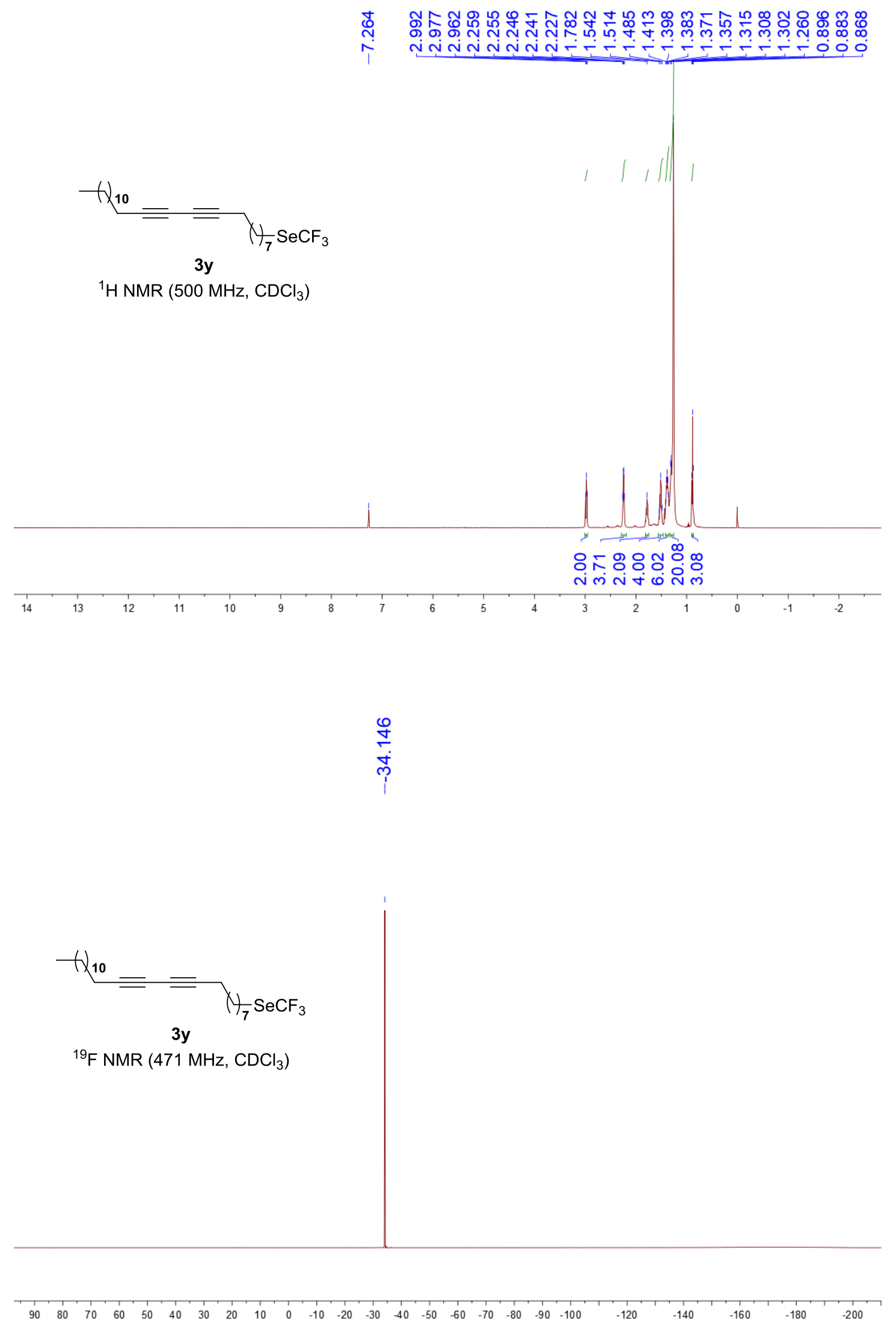



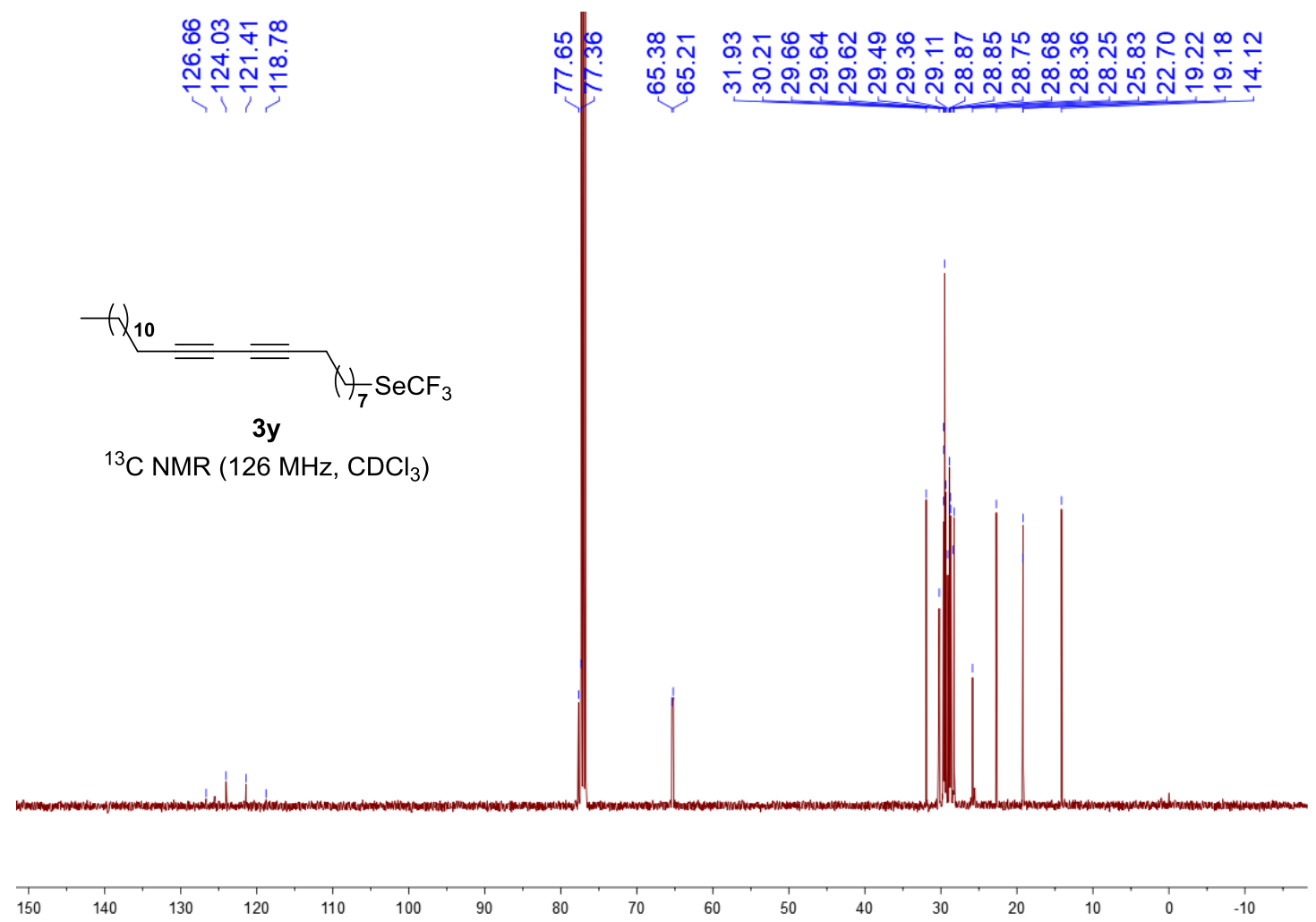

ஸั

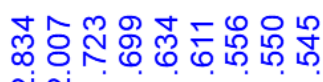

N N N Tr.

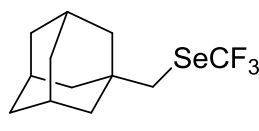

$3 z$

${ }^{1} \mathrm{H}$ NMR $\left(500 \mathrm{MHz}, \mathrm{CDCl}_{3}\right)$ 


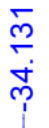

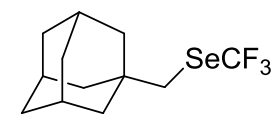

$3 z$

${ }^{19} \mathrm{~F} \mathrm{NMR}\left(471 \mathrm{MHz}, \mathrm{CDCl}_{3}\right)$

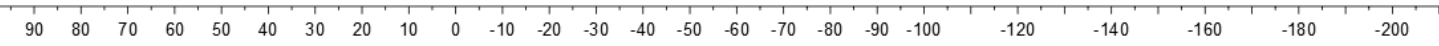

舟

ํํㄱำ

象品

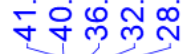

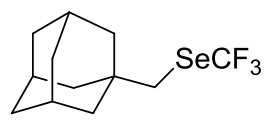

$3 z$

${ }^{13} \mathrm{C} \mathrm{NMR}\left(126 \mathrm{MHz}, \mathrm{CDCl}_{3}\right)$

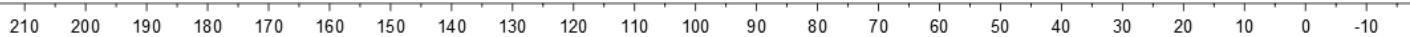



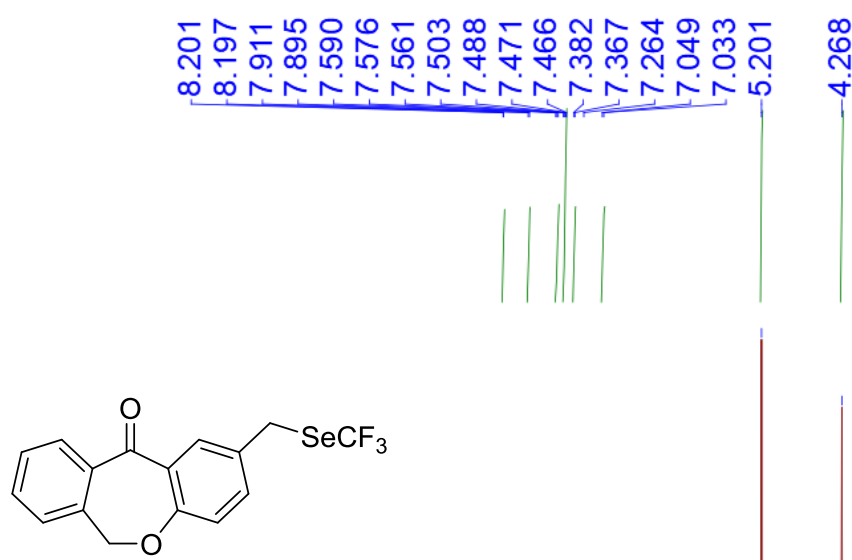

3aa

${ }^{1} \mathrm{H}$ NMR $\left(500 \mathrm{MHz}, \mathrm{CDCl}_{3}\right)$

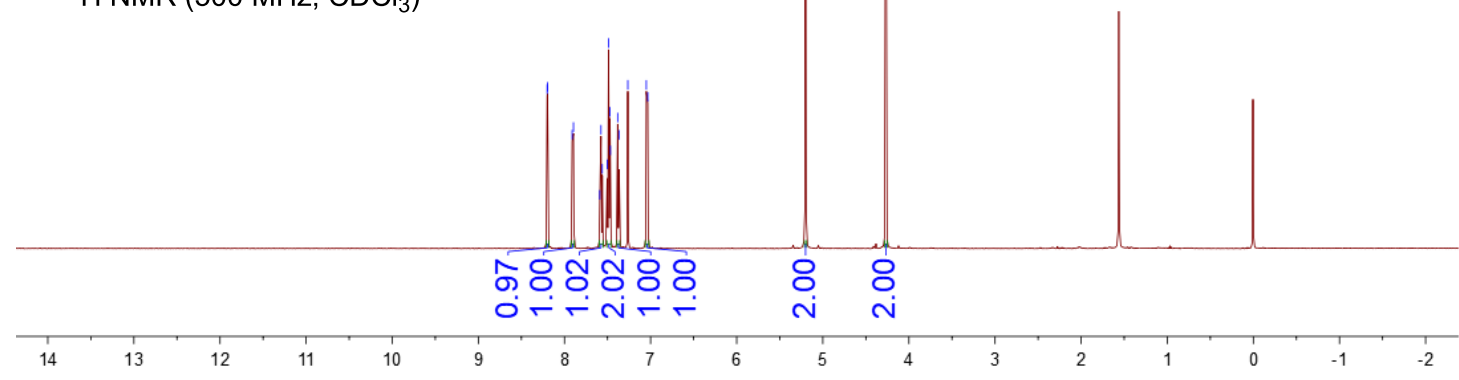

กิ

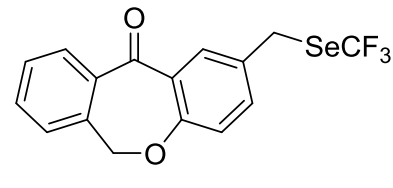

3aa

${ }^{19} \mathrm{~F} \mathrm{NMR}\left(471 \mathrm{MHz}, \mathrm{CDCl}_{3}\right.$ )

\begin{tabular}{llllllllllllllllllllllllllllllll}
\hline 90 & 80 & 70 & 60 & 50 & 40 & 30 & 20 & 10 & 0 & -10 & -20 & -30 & -40 & -50 & -60 & -70 & -80 & -90 & -100 & -120 & -140 & -160 & -180 & -200
\end{tabular} 

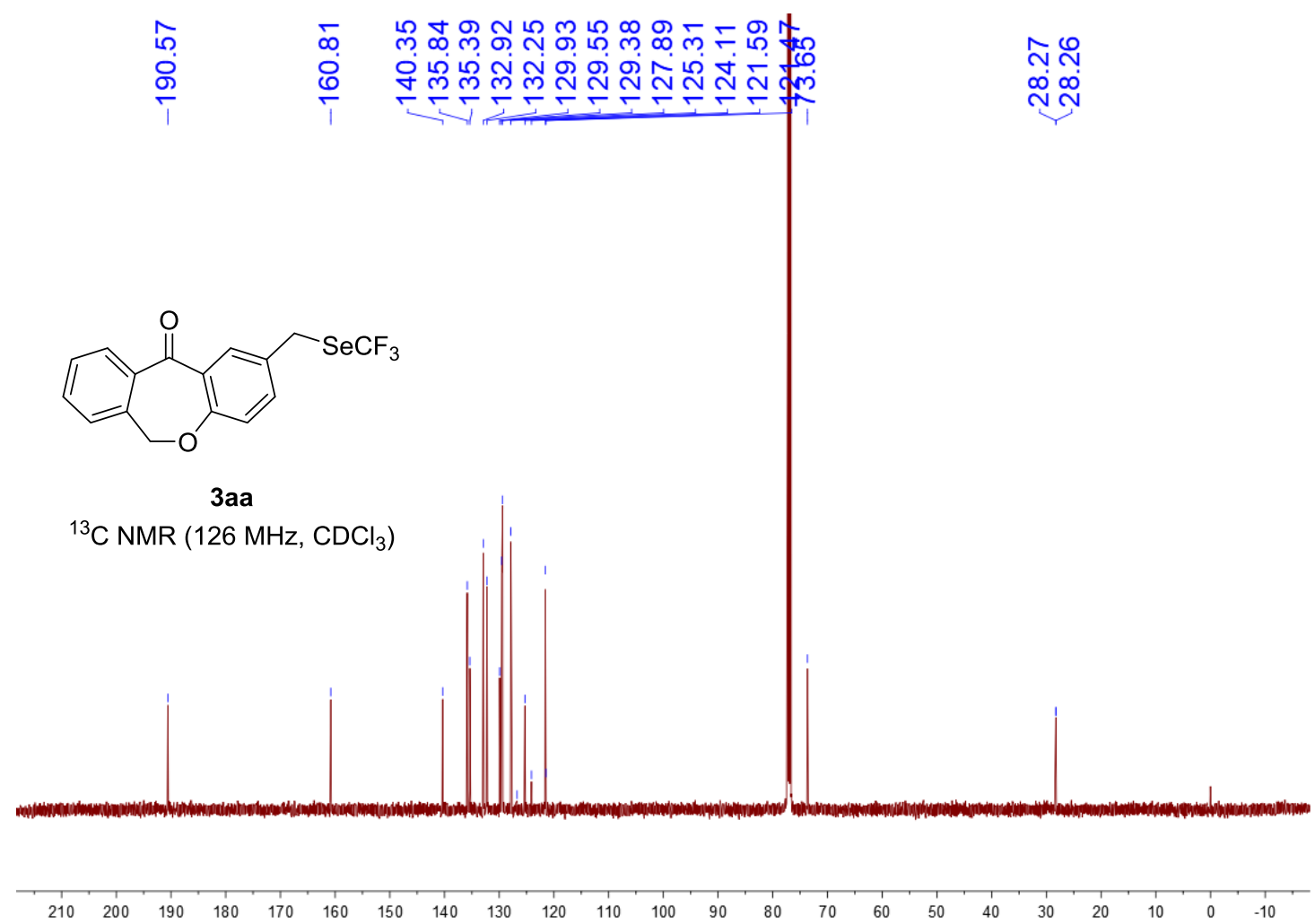

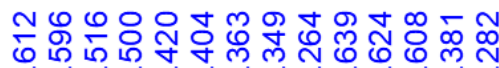

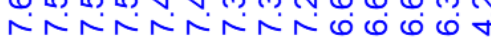
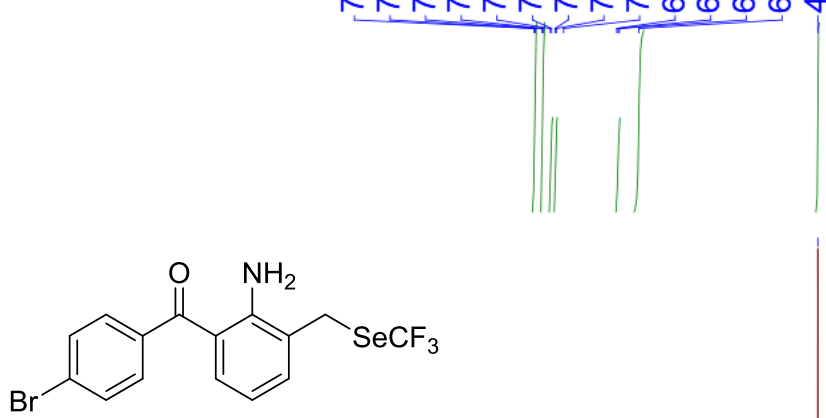

$3 a b$

${ }^{1} \mathrm{H}$ NMR $\left(500 \mathrm{MHz}, \mathrm{CDCl}_{3}\right)$

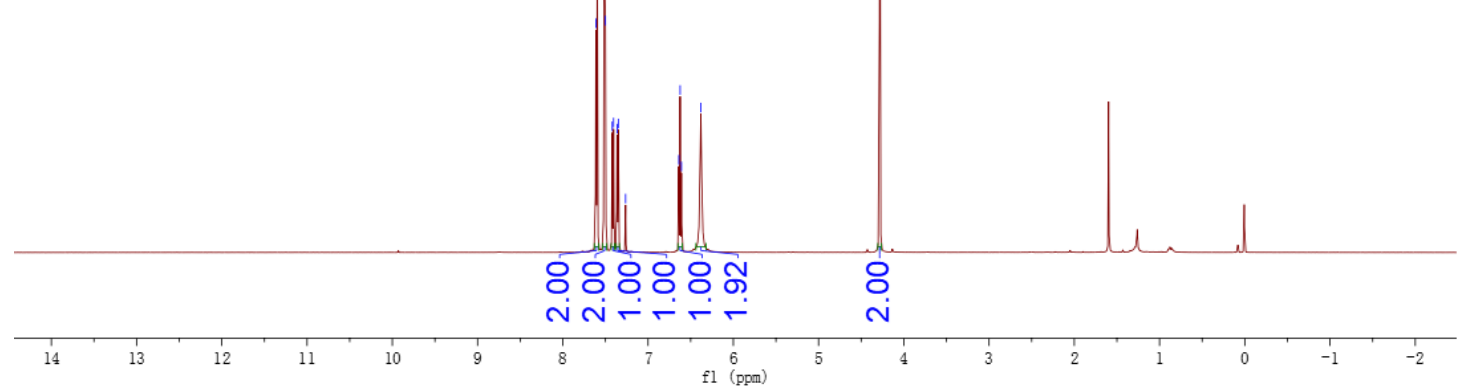




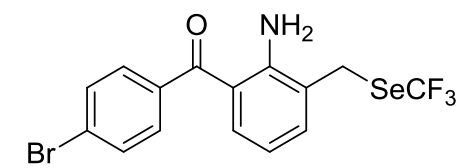

$3 a b$

${ }^{19} \mathrm{~F} \mathrm{NMR}\left(471 \mathrm{MHz}, \mathrm{CDCl}_{3}\right)$

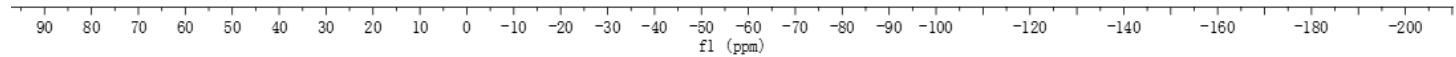

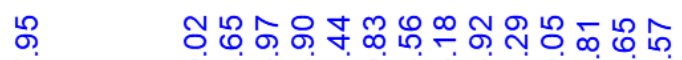

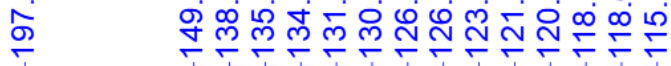

$8 \stackrel{\circ}{\circ}$

ูํำ

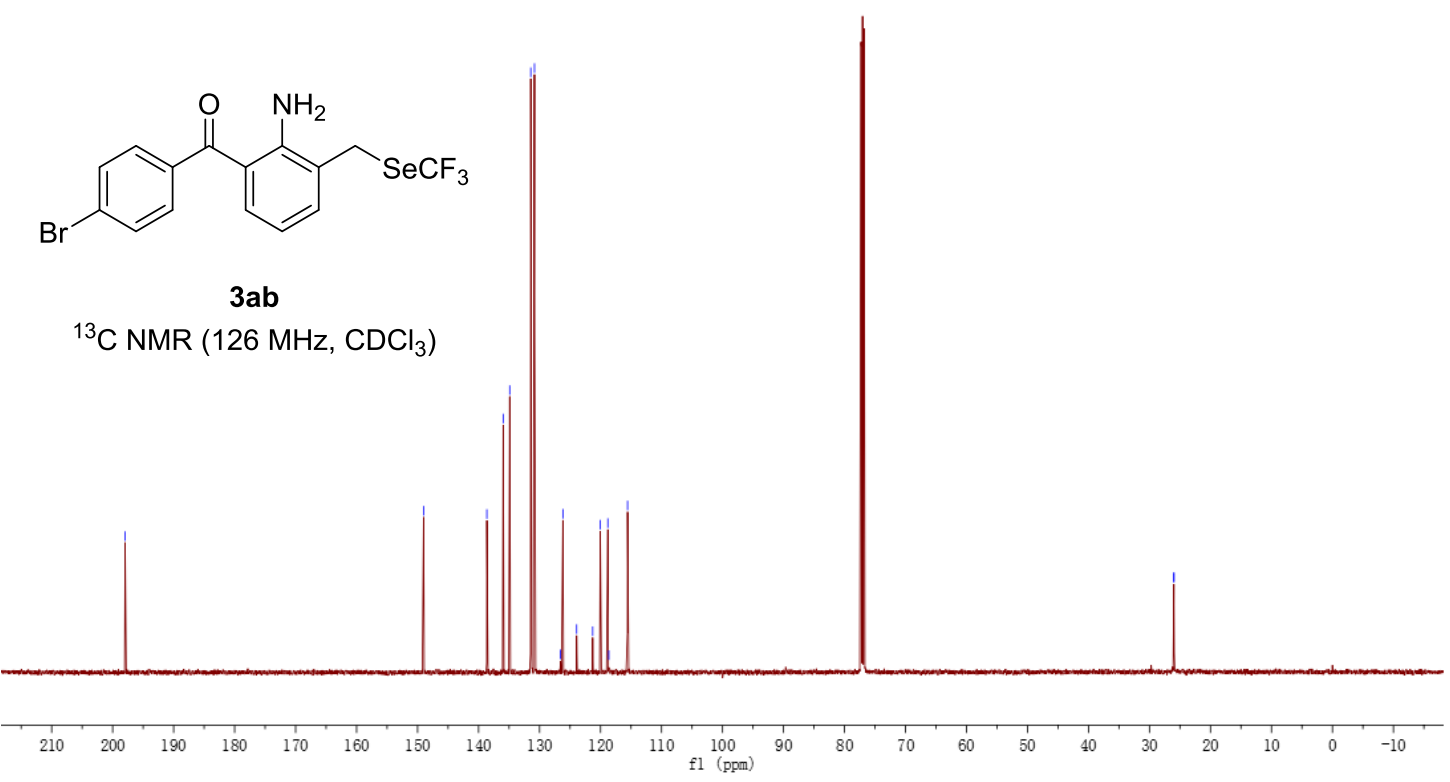




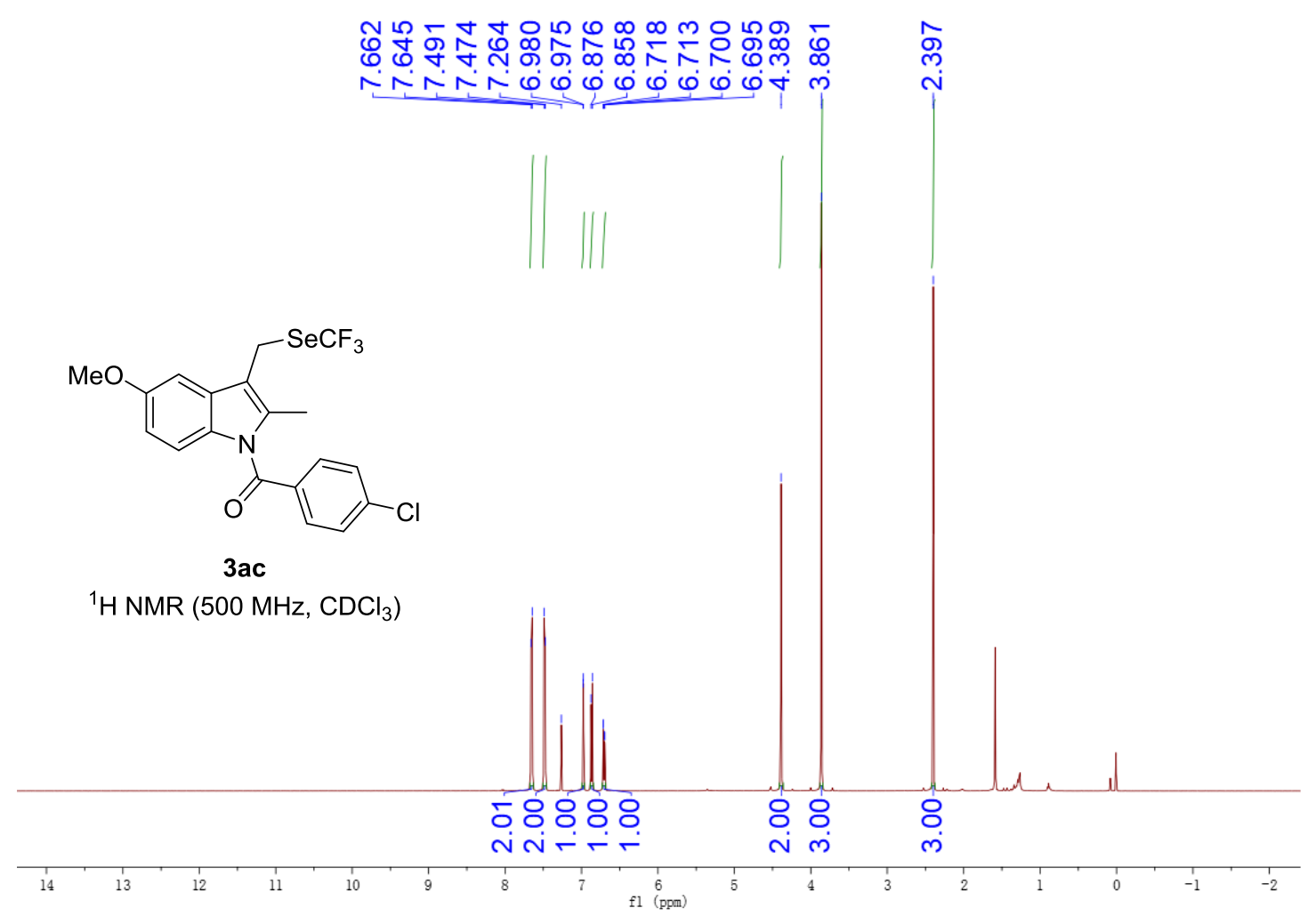

$\stackrel{m}{\stackrel{m}{+}}$

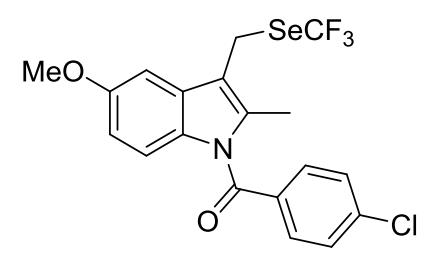

3ac

${ }^{19} \mathrm{~F}$ NMR $\left(471 \mathrm{MHz}, \mathrm{CDCl}_{3}\right)$

$\begin{array}{lllllllllllllllllllllllllllll}90 & 80 & 70 & 60 & 50 & 40 & 30 & 20 & 10 & 0 & -10 & -20 & -30 & -40 & -50 & -60 & -70 & -80 & -90 & -100 & -120 & -140 & -160 & -180 & -200\end{array}$ 

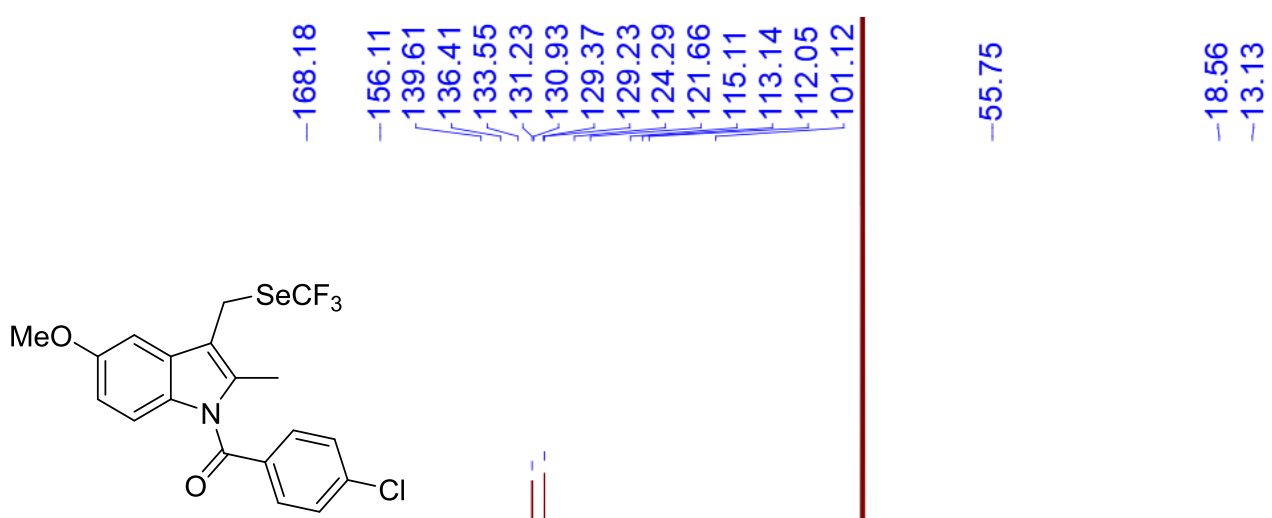

3ac

${ }^{13} \mathrm{C} \mathrm{NMR}\left(126 \mathrm{MHz}, \mathrm{CDCl}_{3}\right)$

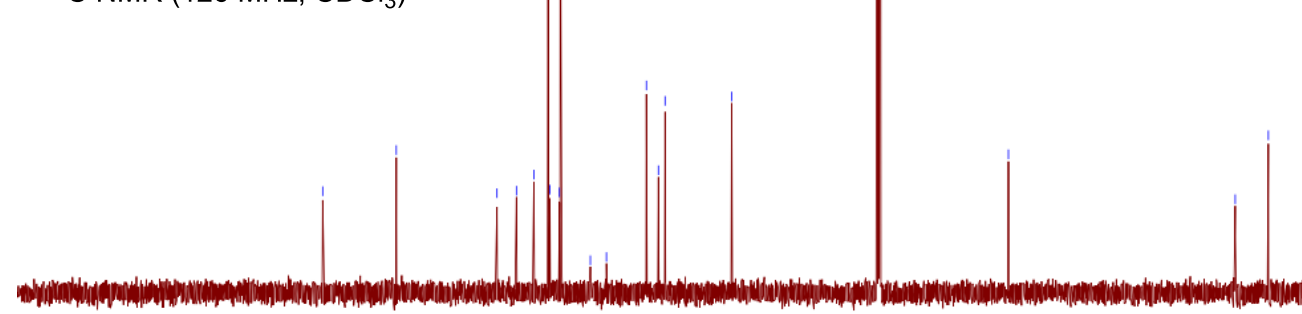

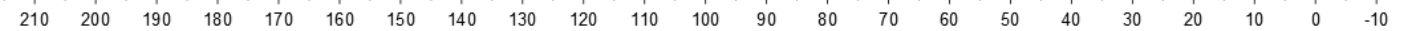

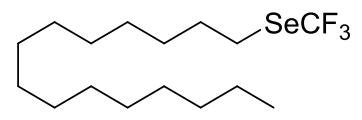

กั

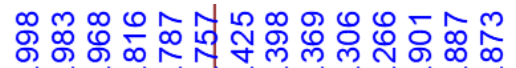

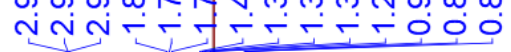

3ad

${ }^{1} \mathrm{H}$ NMR $\left(500 \mathrm{MHz}, \mathrm{CDCl}_{3}\right)$ 


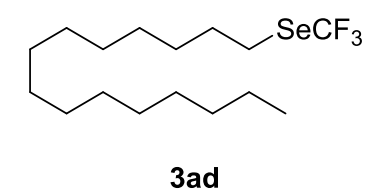

${ }^{19} \mathrm{~F} \mathrm{NMR}\left(471 \mathrm{MHz}, \mathrm{CDCl}_{3}\right)$

ํํํㅇํㅇㅇำ

넌호

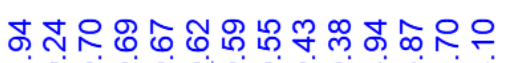

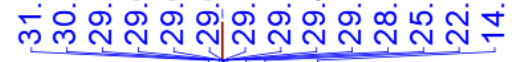

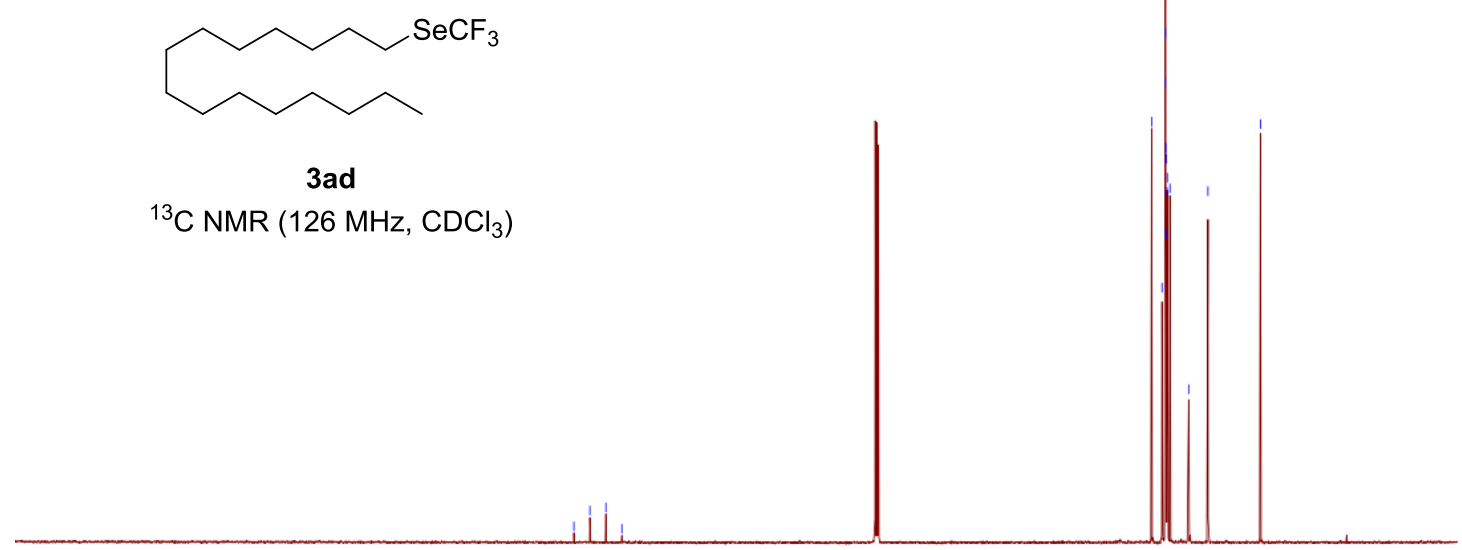

${ }^{13} \mathrm{C}$ NMR $\left(126 \mathrm{MHz}, \mathrm{CDCl}_{3}\right)$

난

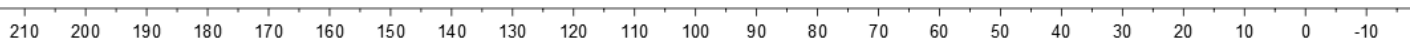


ষ্ণ

N

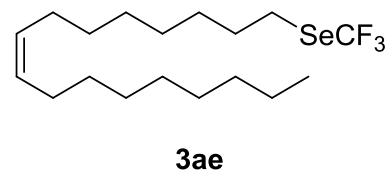

${ }^{1} \mathrm{H}$ NMR $\left(500 \mathrm{MHz}, \mathrm{CDCl}_{3}\right)$

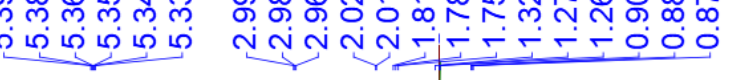

$\underset{⿱ 亠 巾}{\grave{i}}$

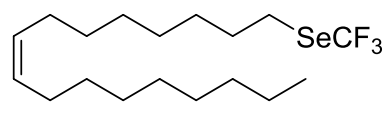

3ae

우유ํ요

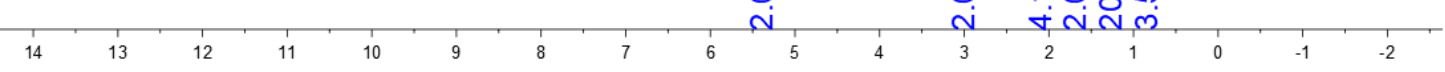

${ }^{19} \mathrm{~F} \mathrm{NMR}\left(471 \mathrm{MHz}, \mathrm{CDCl}_{3}\right)$

$\begin{array}{llllllllllllllllllllllllllllllll} & 80 & 70 & 60 & 50 & 40 & 30 & 20 & 10 & 0 & -10 & -20 & -30 & -40 & -50 & -60 & -70 & -80 & -90 & -100 & -120 & -140 & -160 & -180 & -200\end{array}$ 
으엉웡요

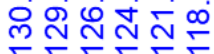

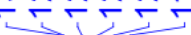

3ae

${ }^{13} \mathrm{C}$ NMR $\left(126 \mathrm{MHz}, \mathrm{CDCl}_{3}\right)$

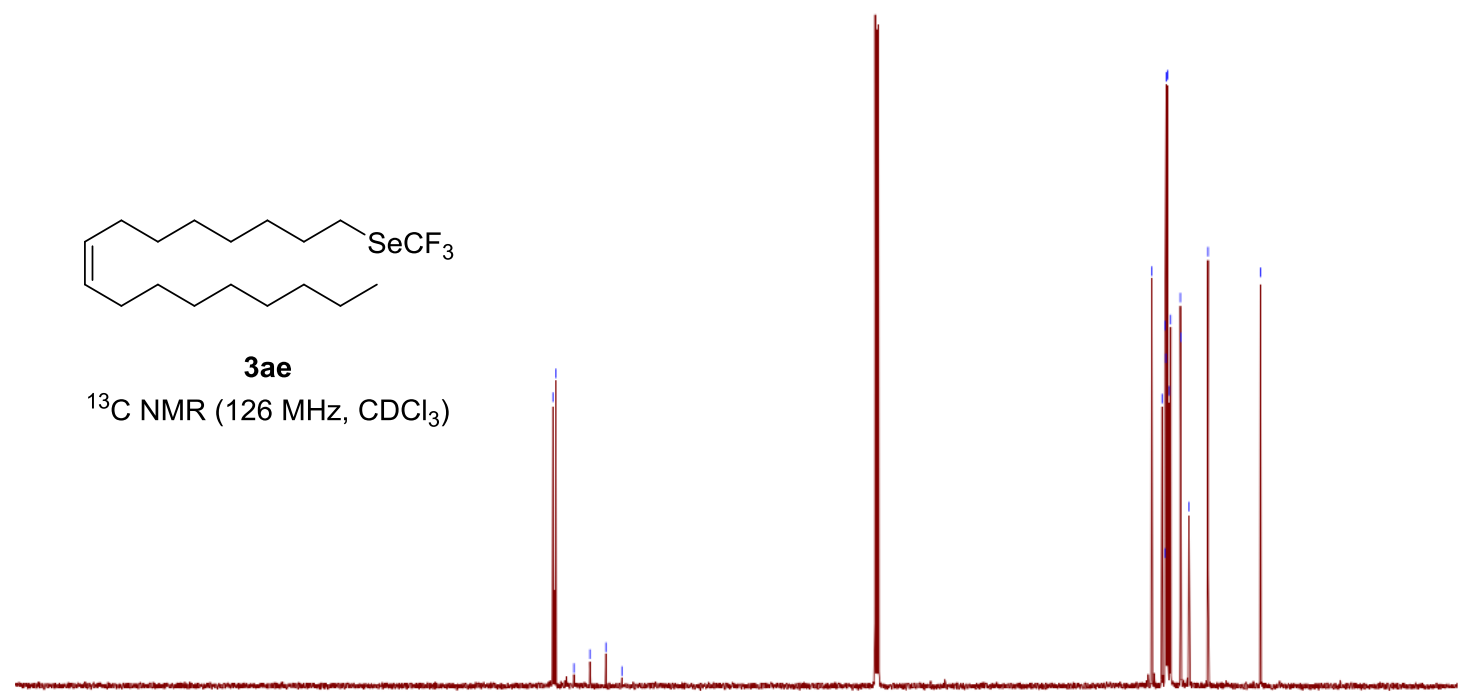

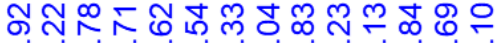

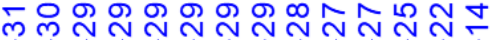

$\begin{array}{lllllllllllllllllllllllllllll} & 210 & 200 & 190 & 180 & 170 & 160 & 150 & 140 & 130 & 120 & 110 & 100 & 90 & 80 & 70 & 60 & 50 & 40 & 30 & 20 & 10 & 0 & -10\end{array}$
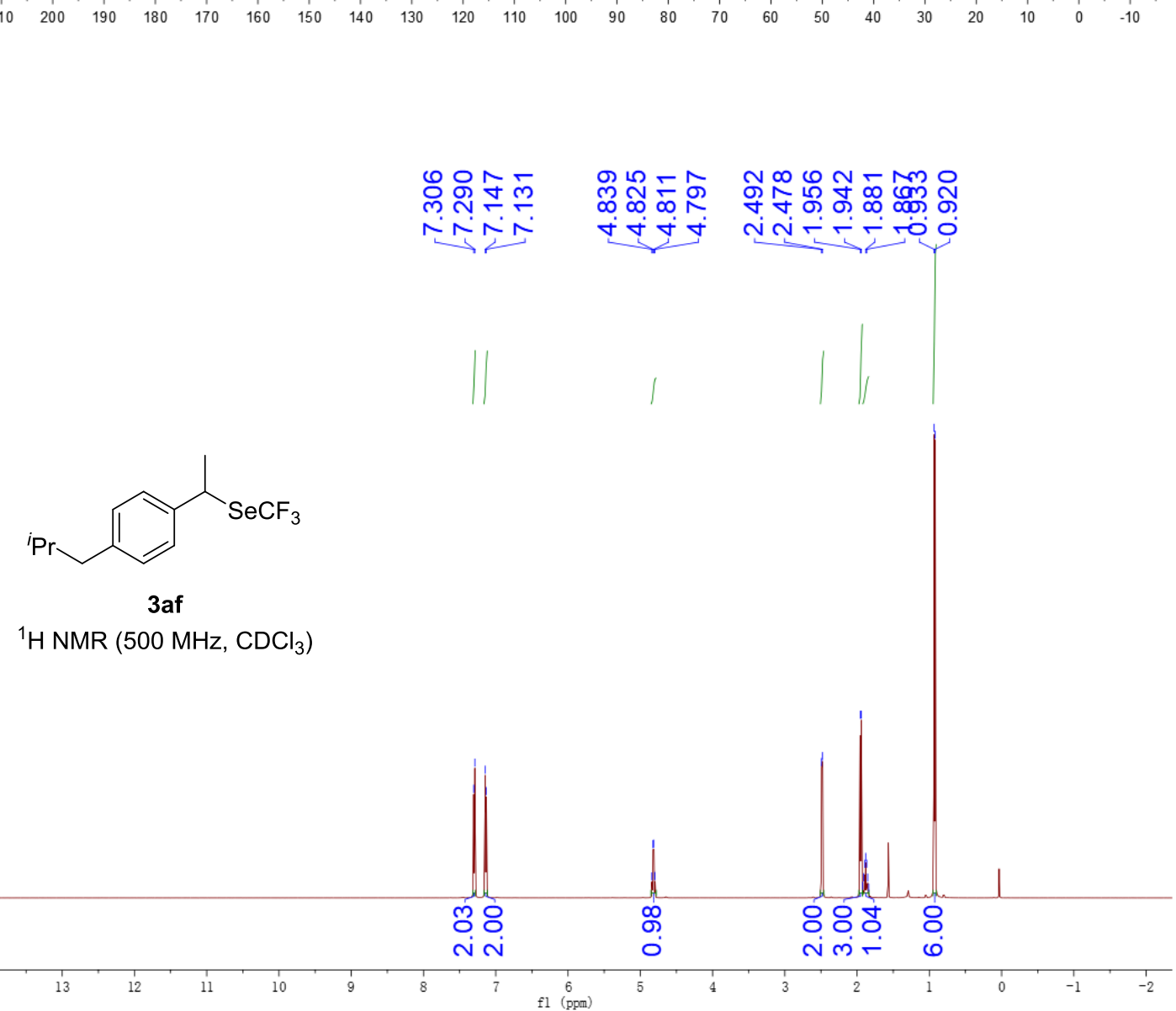


\section{స్م}

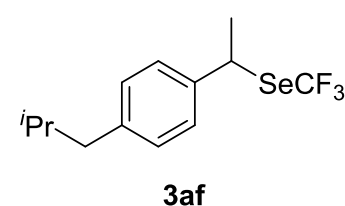

${ }^{19} \mathrm{~F} \mathrm{NMR}\left(471 \mathrm{MHz}, \mathrm{CDCl}_{3}\right)$

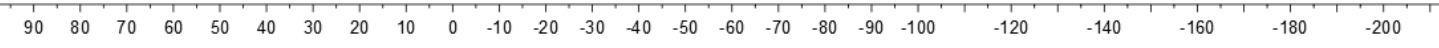

คิ

ช্

수솓ำ

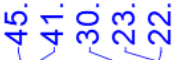

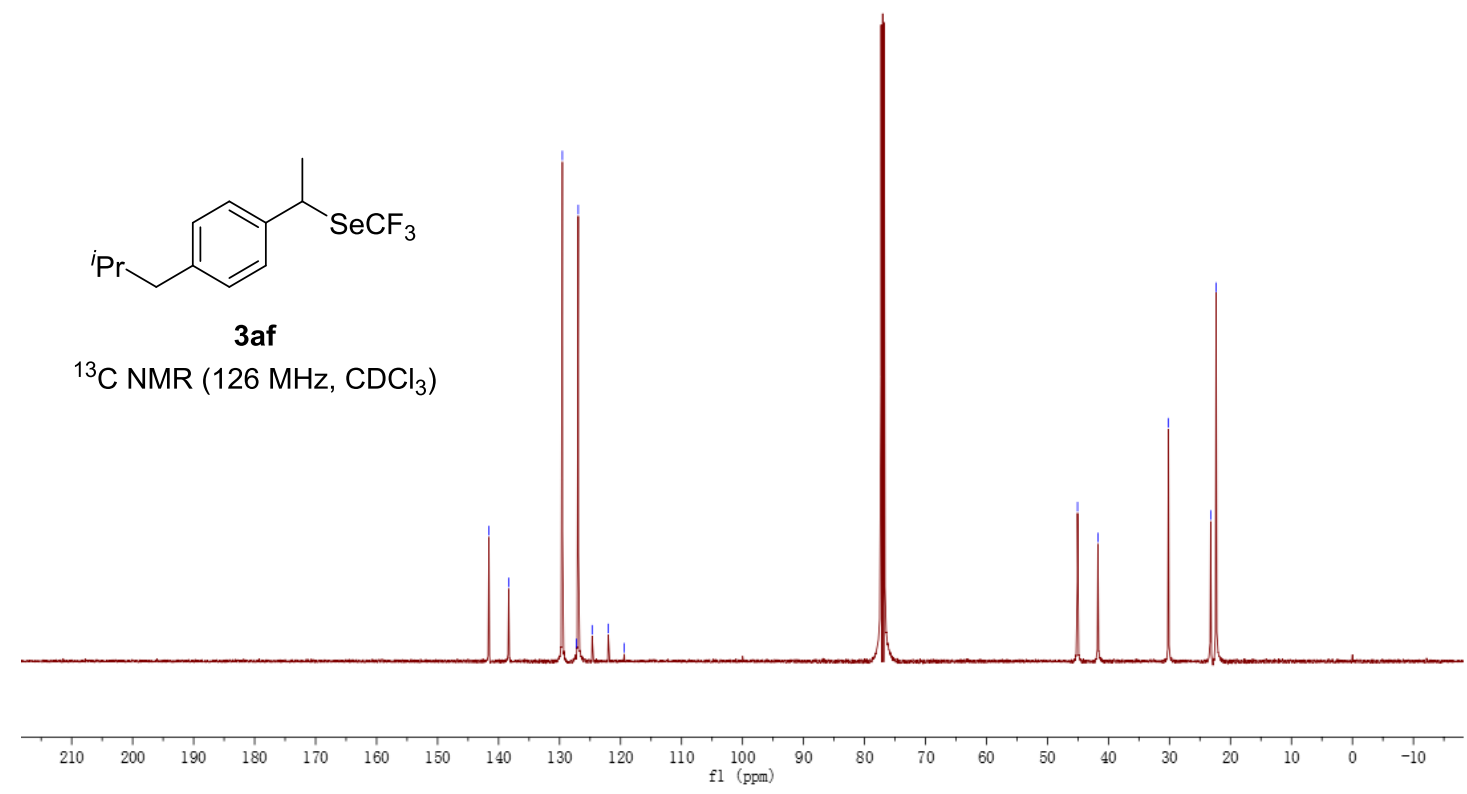



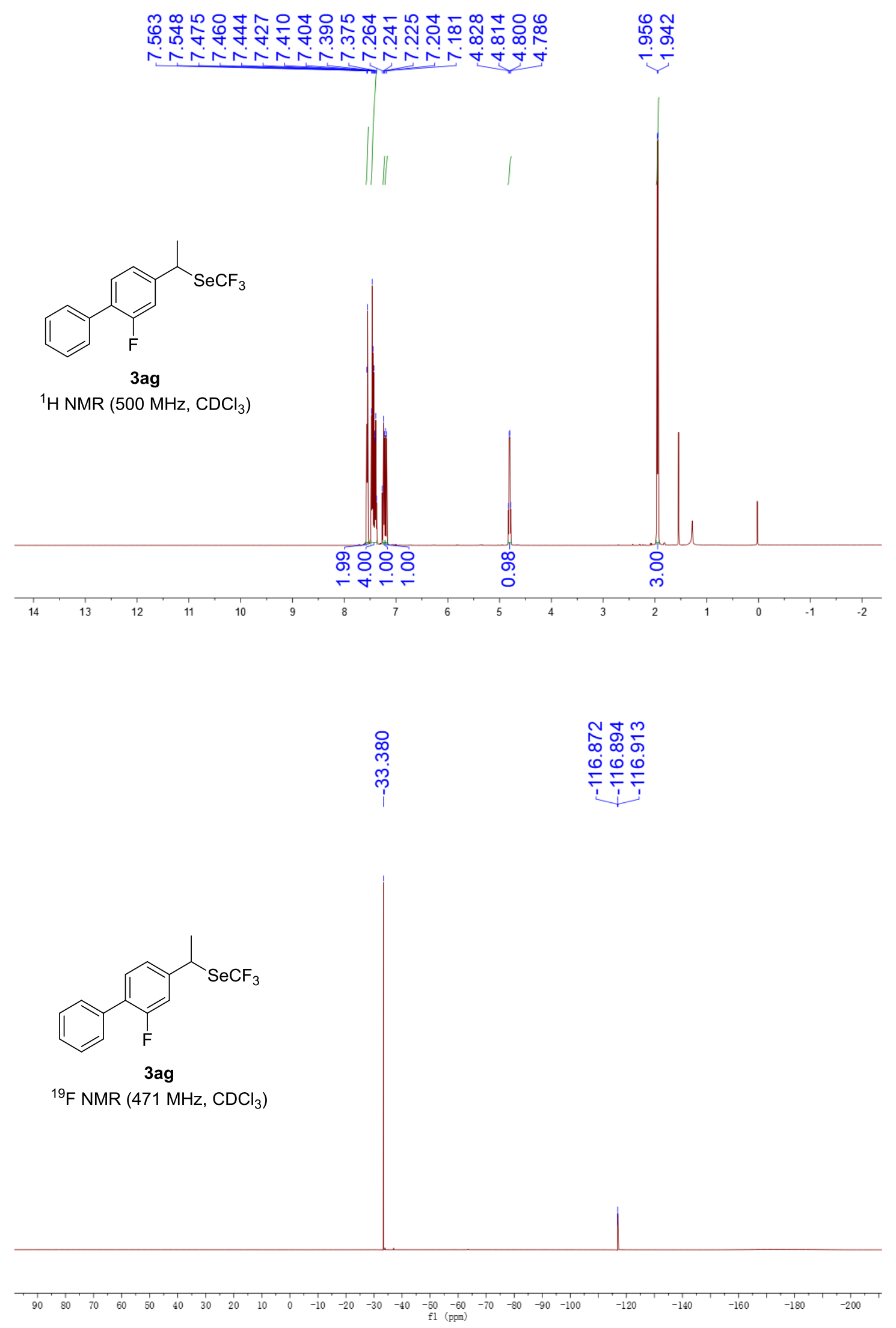


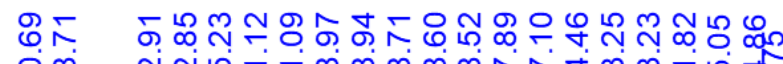

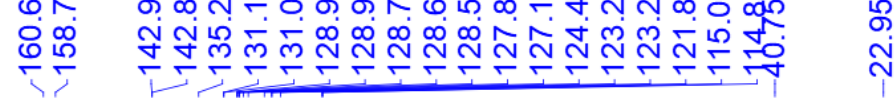
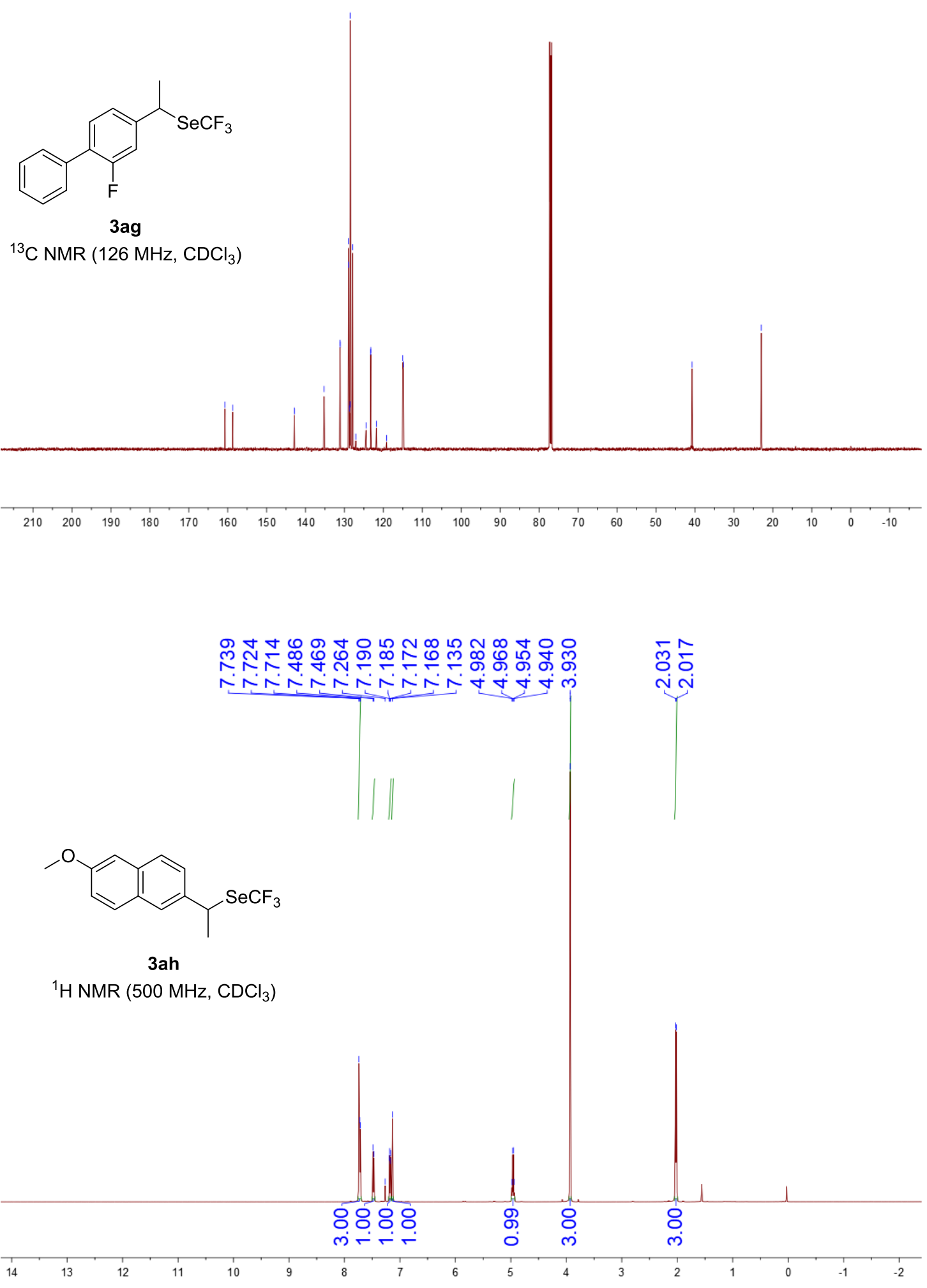


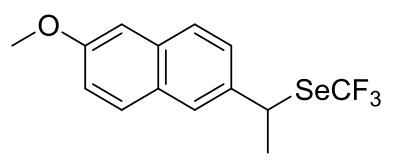

3ah

${ }^{19} \mathrm{~F} \mathrm{NMR}\left(471 \mathrm{MHz}, \mathrm{CDCl}_{3}\right)$

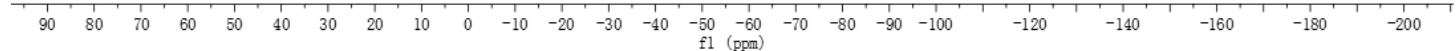

으

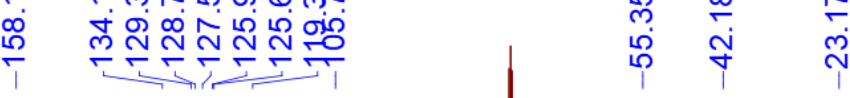

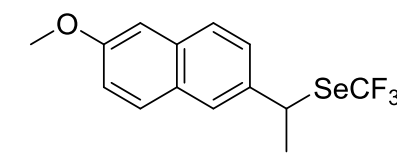

3ah

${ }^{13} \mathrm{C}$ NMR $\left(126 \mathrm{MHz}, \mathrm{CDCl}_{3}\right)$

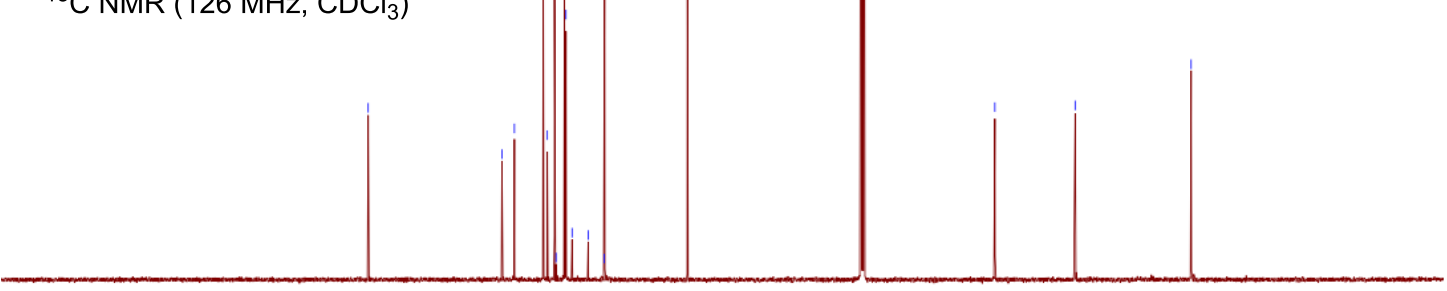

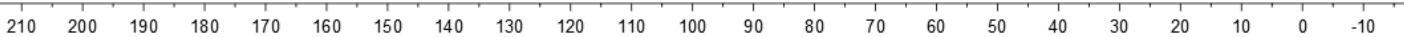



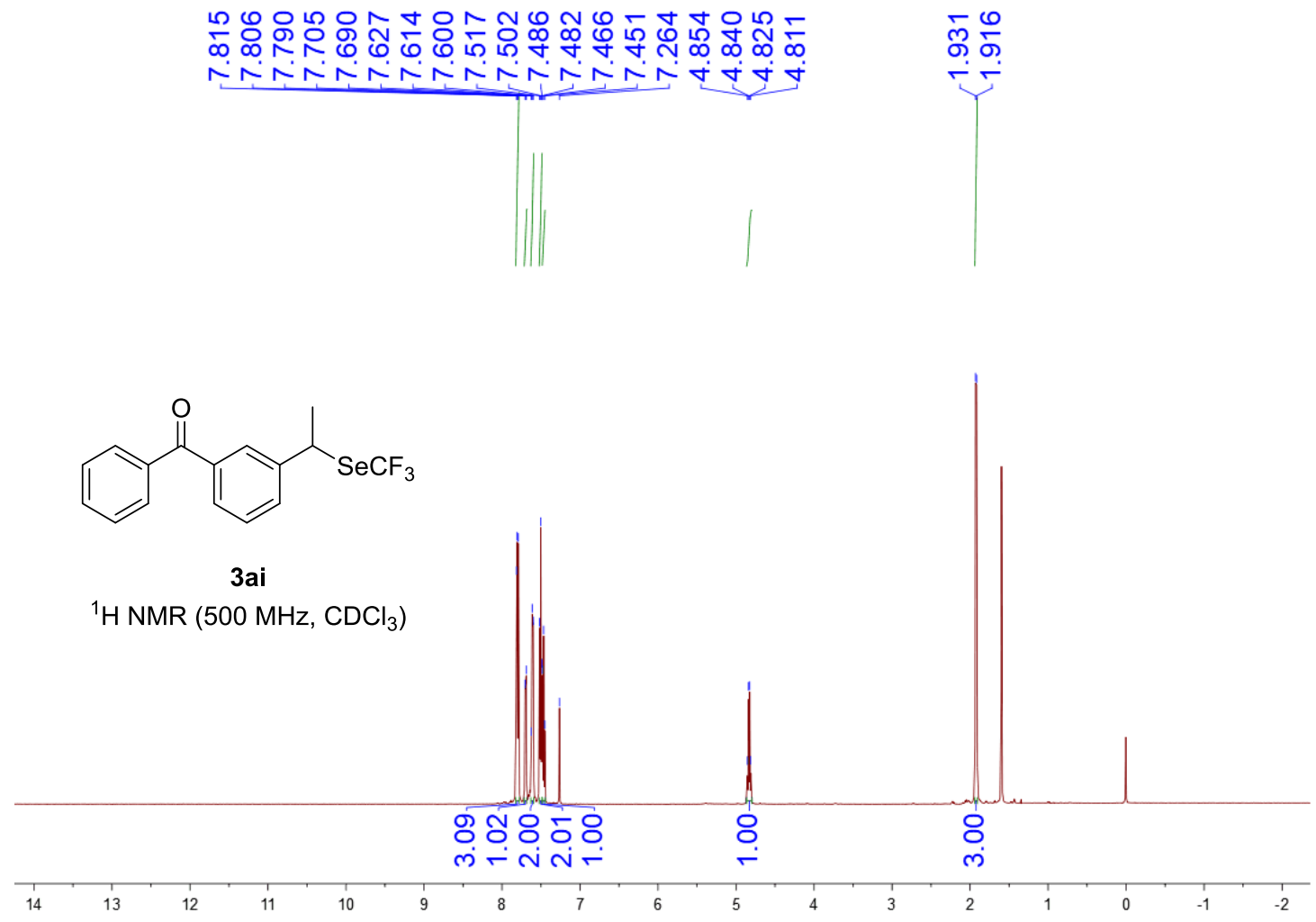

号

m<smiles>CC(c1cccc(C(=O)c2ccccc2)c1)C(F)(F)F</smiles>

3ai

$\left.{ }^{19} \mathrm{~F} \mathrm{NMR} \mathrm{(471} \mathrm{MHz,} \mathrm{CDCl}_{3}\right)$

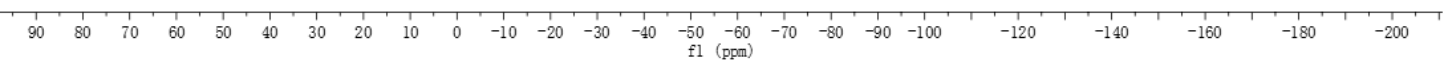




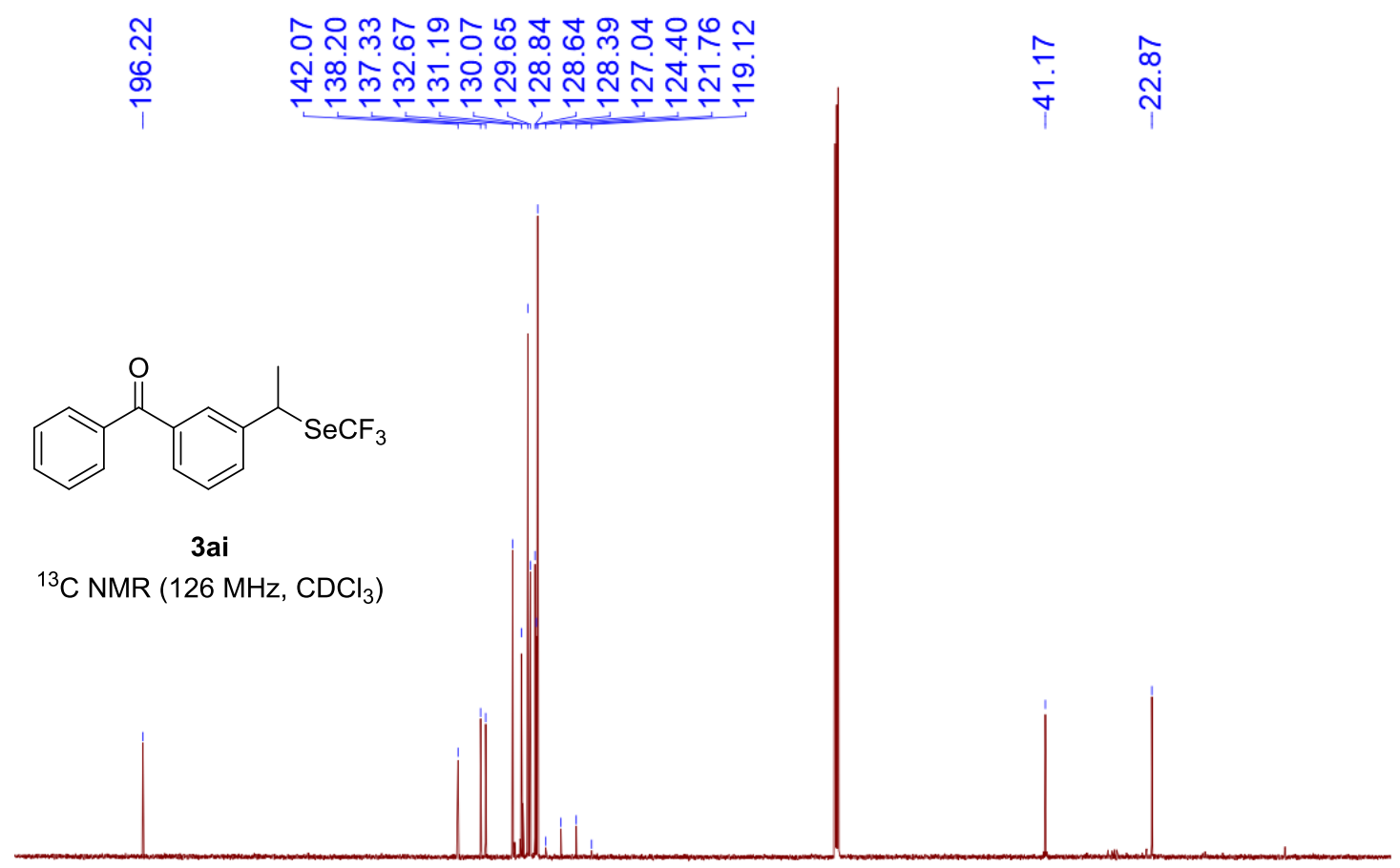

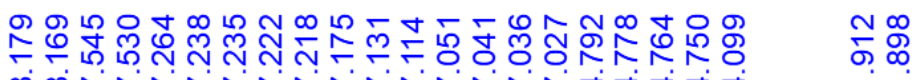

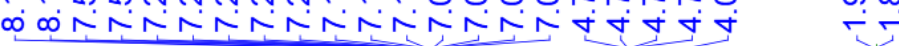

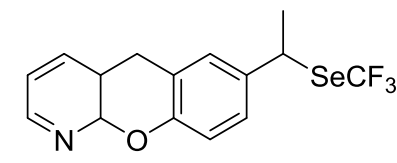

3aj

${ }^{1} \mathrm{H}$ NMR $\left(500 \mathrm{MHz}, \mathrm{CDCl}_{3}\right)$

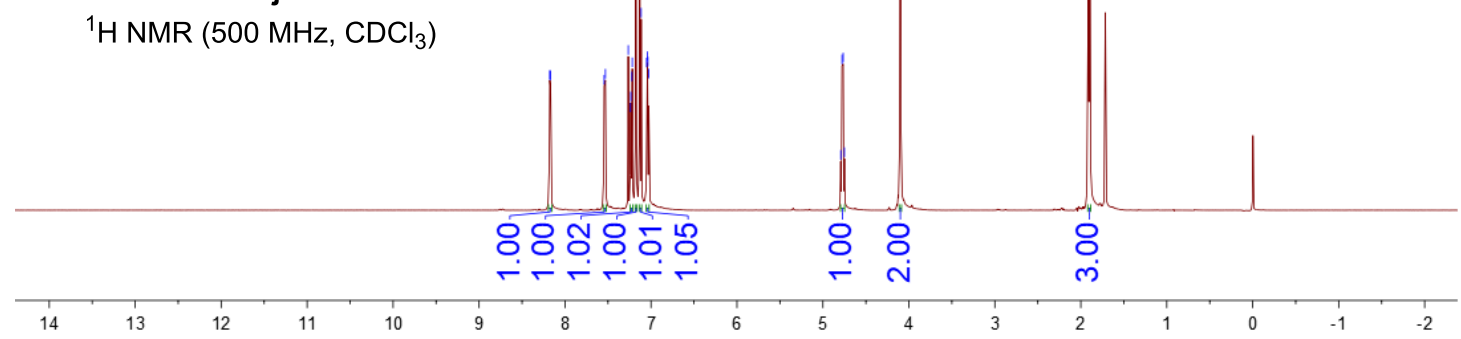




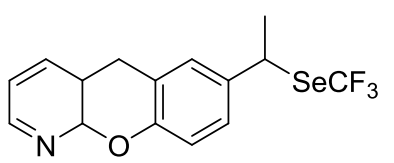

3aj

${ }^{19} \mathrm{~F} \mathrm{NMR}\left(471 \mathrm{MHz}, \mathrm{CDCl}_{3}\right)$

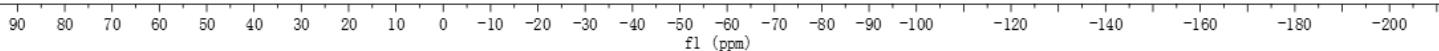

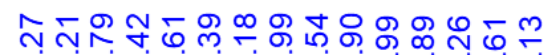

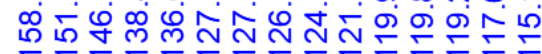

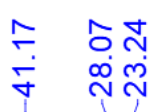

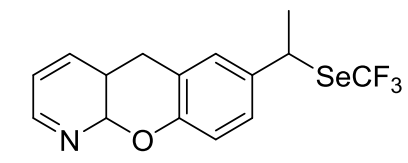

3aj

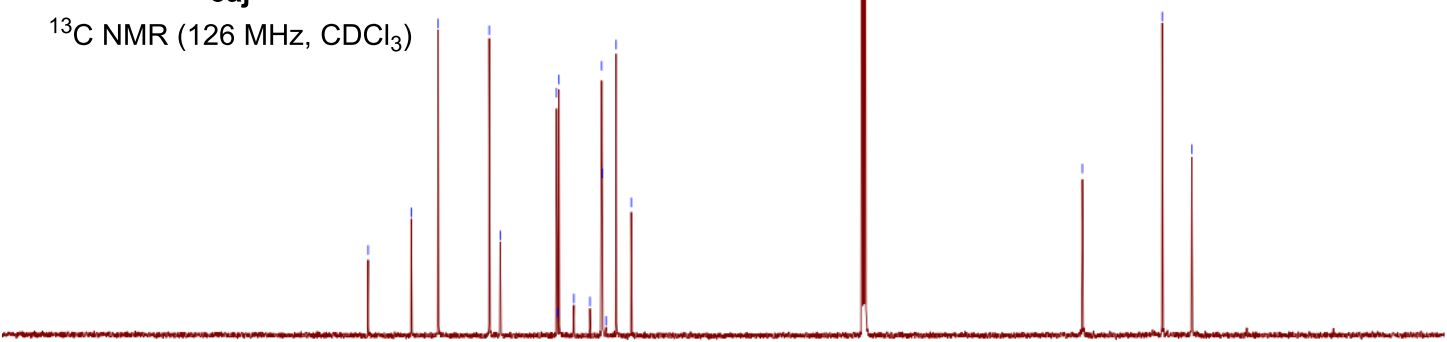

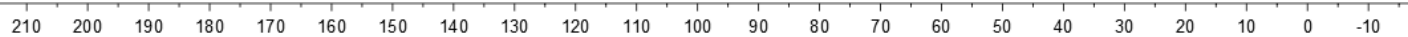




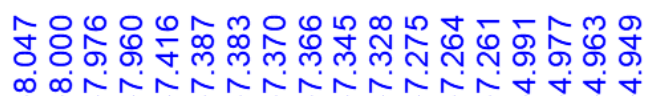

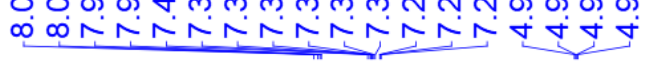
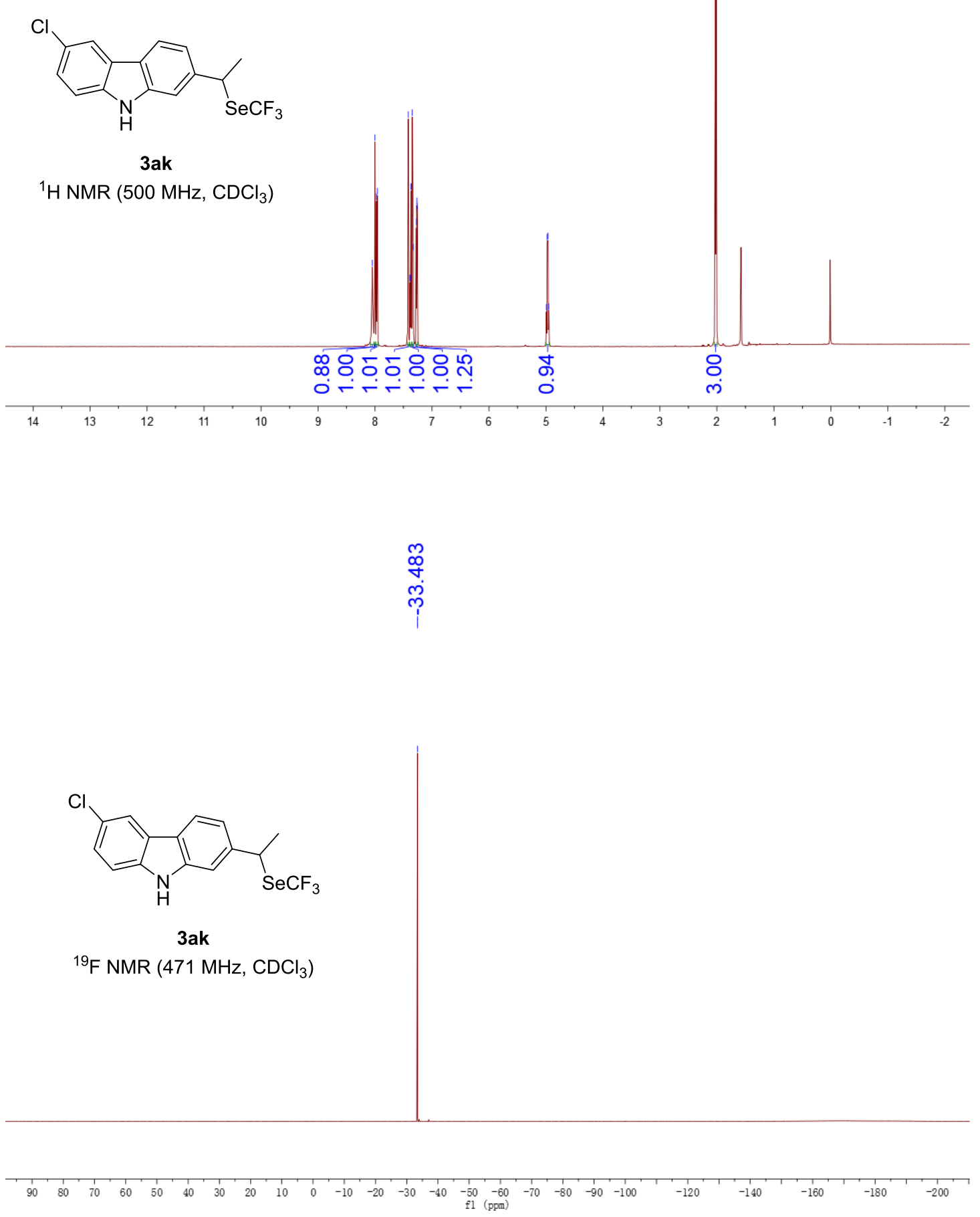

S93 

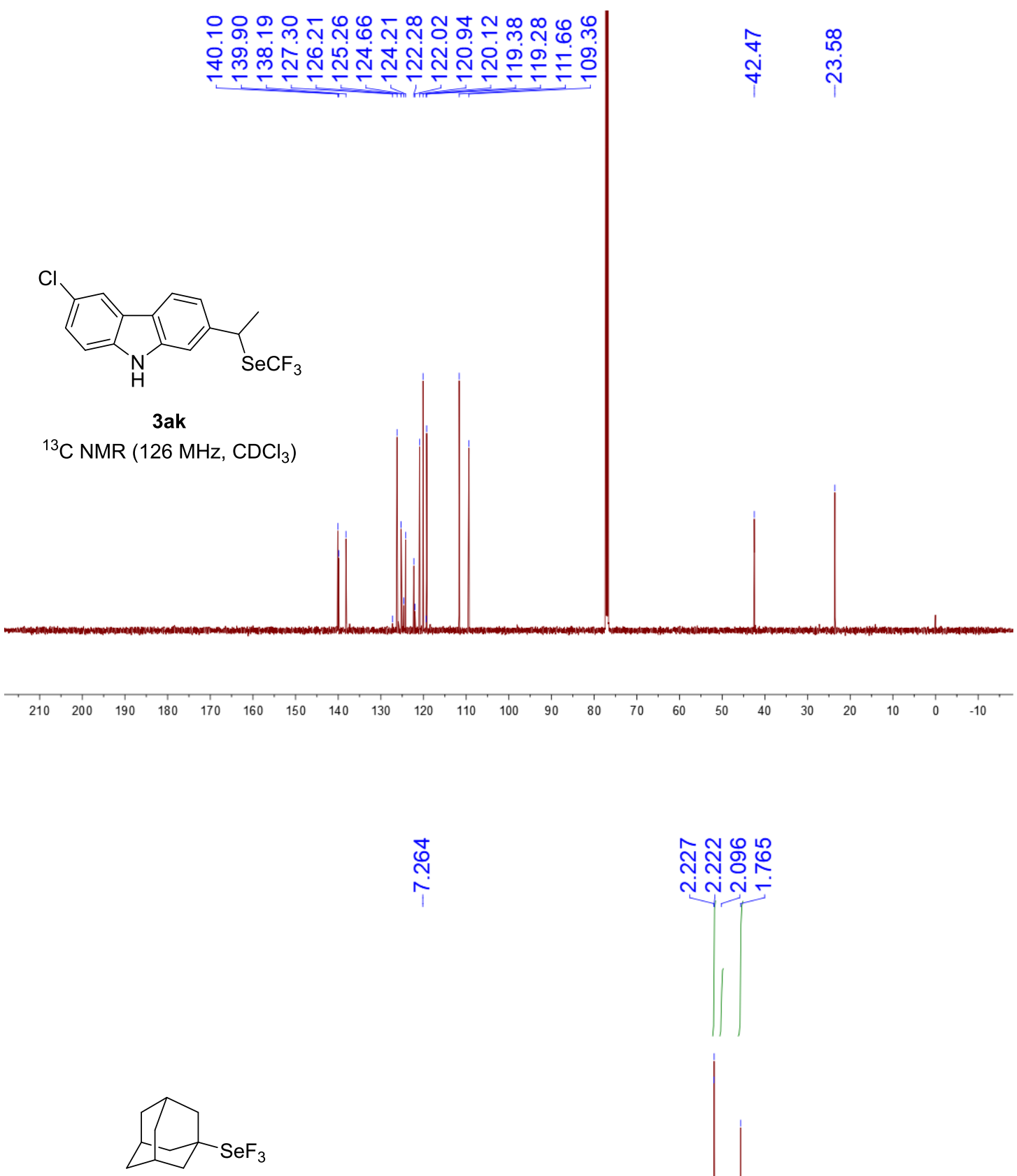

3al

กั

กิ సิด

กำ 


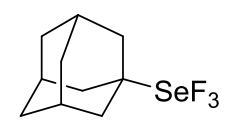

3al

${ }^{19} \mathrm{~F} \mathrm{NMR}\left(471 \mathrm{MHz}, \mathrm{CDCl}_{3}\right)$

\begin{tabular}{lllllllllllllllllllllllllllllllllll}
\hline 90 & 80 & 70 & 60 & 50 & 40 & 30 & 20 & 10 & 0 & -10 & -20 & -30 & -40 & -50 & -60 & -70 & -80 & -90 & -100 & -120 & -140 & -160 & -180 & -200 & 1
\end{tabular}

$\infty_{\infty} \underset{\infty}{\infty} \infty$

ํํำ ํํㅇ

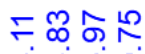

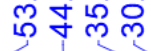

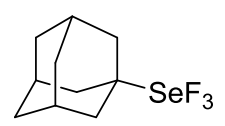

3al

${ }^{13} \mathrm{C}$ NMR $\left(1261 \mathrm{MHz}, \mathrm{CDCl}_{3}\right)$

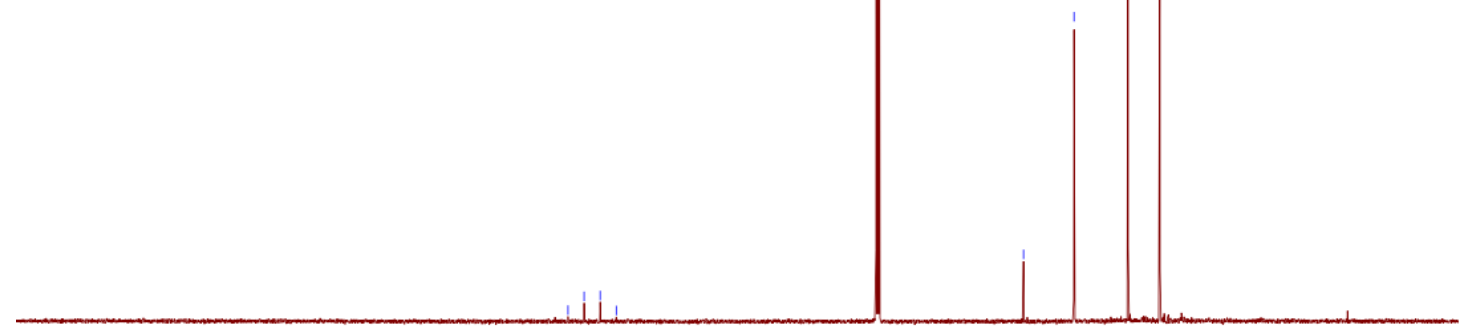

$\begin{array}{llllllllllllllllllllllll}1 & 1 & 200 & 190 & 180 & 170 & 160 & 150 & 140 & 130 & 120 & 110 & 100 & 90 & 80 & 70 & 60 & 50 & 40 & 30 & 20 & 10 & 0 & -10\end{array}$ 